\title{
Sludge Pretreatment Chemistry Evaluation: Enhanced Sludge Washing Separation Factors
}

\author{
N. G. Colton
}

March 1995

Prepared for the U.S. Department of Energy under Contract DE-AC06-76RLO 1830

Pacific Northwest Laboratory Operated for the U.S. Department of Energy by Battelle Memorial Institute 


\title{
DISCLAIMER
}

This report was prepared as an account of work sponsored by an agency of the United States Government. Neither the United States Government nor any agency thereof, nor Battelle Memorial Institute, nor any of their employees, makes any warranty, expressed or implied, or assumes any legal liability or responsibility for the accuracy, completeness, or usefulness of any information, apparatus, product, or process disclosed, or represents that its use would not infringe privately owned rights. Reference herein to any specific commercial product, process, or service by trade name, trademark, manufacturer, or otherwise does not necessarily constitute or imply its endorsement, recommendation, or favoring by the United States Government or any agency thereof, or Battelle Memorial Institute. The views and opinions of authors expressed herein do not necessarily state or reflect those of the United States Government or any agency thereof.

\author{
PACIFIC NORTHWEST LABORATORY \\ operated by \\ BATTELLE MEMORIAL INSTITUTE \\ for the \\ UNITED STATES DEPARTMENT OF ENERGY \\ under Contract DE-AC06-76RLO 1830
}

Printed in the United States of America

Available to DOE and DOE contractors from the

Office of Scientific and Technical Information; P.O. Box 62, Oak Ridge, TN 37831; prices available from (615) 576-8401. FTS 626-8401.

Available to the public from the National Technical Information Service,

U.S. Department of Commerce, 5285 Port Royal Rd., Springfield, VA 22161. 
PNL-10512

\section{Sludge Pretreatment Chemistry Evaluation: Enhanced Sludge Washing Separation Factors}

N.G. Colton

March 1995

Prepared for the U.S. Department of Energy under Contract DE-AC-06-76RLO 1830

Pacific Northwest Laboratory

Richland, Washington 99352 


\section{Summary}

This report presents the work conducted in Fiscal Year 1994 by the Sludge Pretreatment Chemistry Evaluation Subtask for the Tank Waste Remediation System (TWRS) Tank Waste Treatment Science Task. The main purpose of this task, led by Pacific Northwest Laboratory ${ }^{(a)}$, is to provide the technical basis and scientific understanding to support TWRS baseline decisions and actions, such as the development of an enhanced sludge washing process to reduce the volume of waste that will require high-level waste (HLW) vitrification.

The effect of this enhanced sludge washing strategy on final waste volumes is evaluated in systems engineering studies by using assumptions about the chemical and physical behavior of the wastes. Included in the chemical assumptions are wash and leach factors, or the fraction of each waste component that will dissolve or will be leached from waste streams during various processing steps. Current evaluations use wash factors and waste stream compositions derived from the overall single-shell tank (SST) and double-shell tank (DST) waste inventories, i.e., idealized blends of SST and DST tank wastes. In actuality, wastes will be blended in batches that could result in larger-thananticipated HLW volumes. Evaluating the effect of different blending strategies on HLW volumes requires that pretreatment scenarios based on specific tank or waste-type inventories also be evaluated. For that evaluation, wash factors for these tank/waste-type inventories need to be established.

One objective within the Sludge Pretreatment Chemistry Evaluation Subtask was to establish wash factors for various SST sludges. First, analytical data were compiled from existing tank waste characterization reports. These data were summarized on tank-specific worksheets that provided a uniform format for reviewing and comparing data, as well as the means to verify whether the data set for each tank was complete. Worksheets were completed for 27 SST wastes. The analytical water wash data provided tank-specific information about the fraction of each component that dissolves with water, i.e., an estimate of tank-specific wash factors for evaluating tank-by-tank processing. These wash data were then used collectively to evaluate some of the wash factors that are assumed for the overall SST waste inventory; specifically, wash factors for elements that would be found primarily in sludges. The final step in this study was to incorporate the characterization and wash factor data into a spreadsheet that provides insight into the effect of enhanced sludge washing on individual tank sludges as well as for groups of sludges that may be representative of different waste types.

Spreadsheet results include the estimated mass and percentage of each element that would be removed with washing and leaching. Furthermore, estimated compositions are given of the final wash and leach streams and residual solids, in terms of both concentration and dry weight percent.

(a) Pacific Northwest Laboratory is operated for the U.S. Department of Energy by Battelle Memorial Institute under Contract DE-AC06-76RLO 1830. 


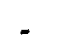




\section{Contents}

Summary $\ldots \ldots \ldots \ldots \ldots \ldots \ldots \ldots \ldots \ldots \ldots \ldots \ldots \ldots \ldots \ldots$ iii

Acknowledgements $\ldots \ldots \ldots \ldots \ldots \ldots \ldots \ldots \ldots \ldots \ldots \ldots \ldots \ldots \ldots$

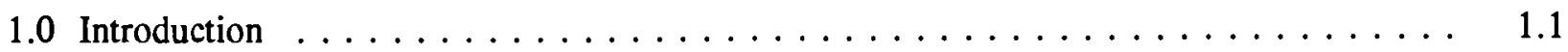

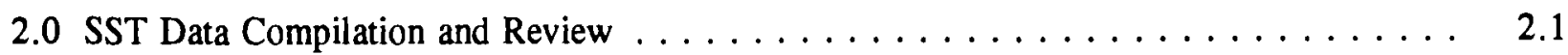

2.1 Key for Interpreting Characterization Data Summary Worksheets . . . . . . . 2.3

\begin{tabular}{|c|c|}
\hline A-102 & $\ldots \ldots \ldots \ldots \ldots \ldots \ldots \ldots \ldots$ \\
\hline A-103 & 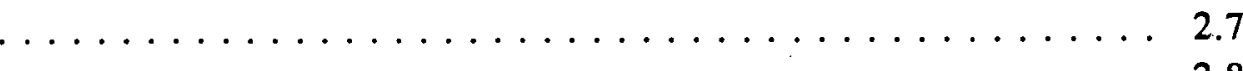 \\
\hline A-106 & $\ldots \ldots \ldots \ldots \ldots \ldots \ldots \ldots \ldots \ldots \ldots \ldots$ \\
\hline B-110 & 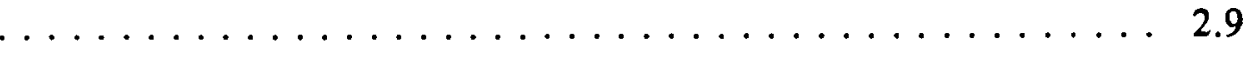 \\
\hline 8-111 & ${ }^{\prime}$ \\
\hline B-201 & $\ldots \ldots \ldots \ldots \ldots \ldots \ldots \ldots$ \\
\hline BX-104 & $\ldots \ldots \ldots \ldots \ldots \ldots$ \\
\hline BX-105 & $\ldots \ldots \ldots \ldots \ldots \ldots \ldots \ldots \ldots \ldots \ldots \ldots \ldots$ \\
\hline BX-107 & 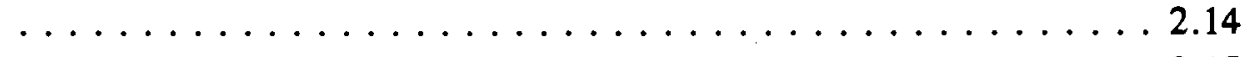 \\
\hline C-103 & 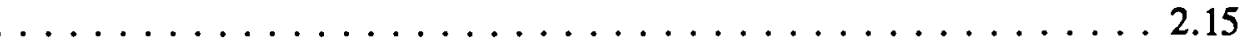 \\
\hline C-104 & 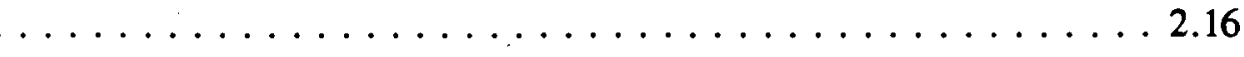 \\
\hline C-105 & $\ldots \ldots \ldots \ldots \ldots \ldots \ldots \ldots$ \\
\hline C-106 & $\ldots \ldots \ldots \ldots \ldots \ldots \ldots \ldots$ \\
\hline C-109 & $\ldots \ldots \ldots \ldots \ldots \ldots$ \\
\hline C-110 & 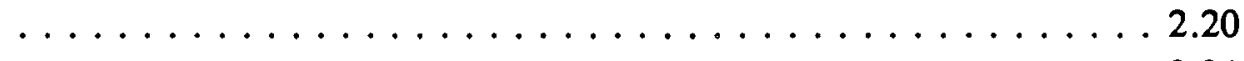 \\
\hline C-112 & 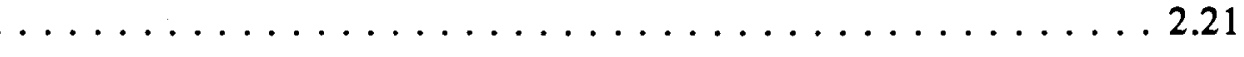 \\
\hline S-104 & 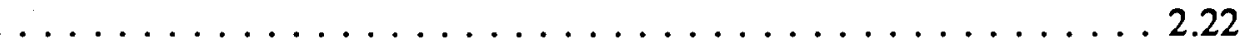 \\
\hline $\mathrm{T}-102$ & 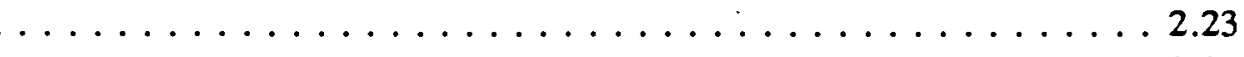 \\
\hline T-104 & 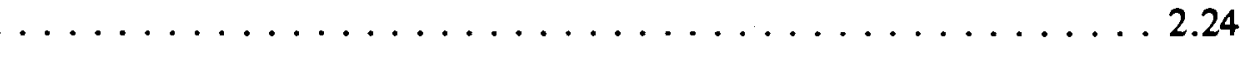 \\
\hline $\mathrm{T}-107$ & 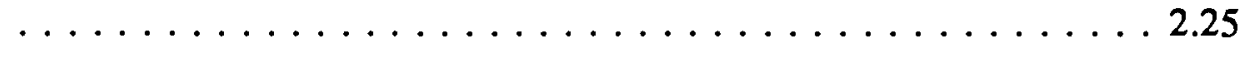 \\
\hline $\mathrm{T}-111$ & 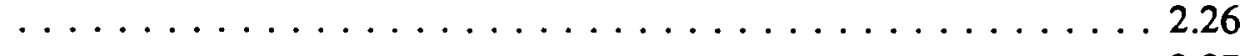 \\
\hline TY-101 & 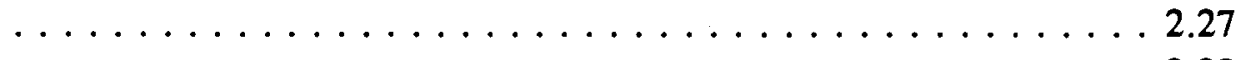 \\
\hline TY-103 & 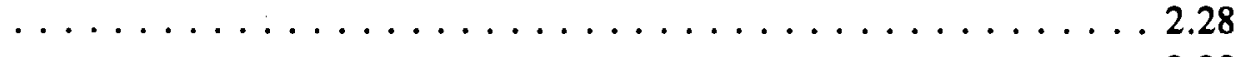 \\
\hline TY-104 & 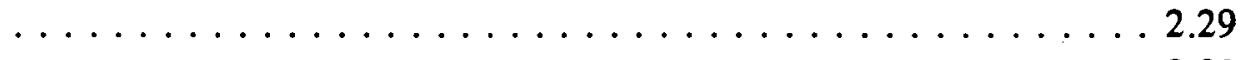 \\
\hline TY-105 & 2.30 \\
\hline $\begin{array}{l}\text { TY-106 } \\
\mathrm{U}-110\end{array}$ & $\cdots \cdots \cdots$ \\
\hline
\end{tabular}

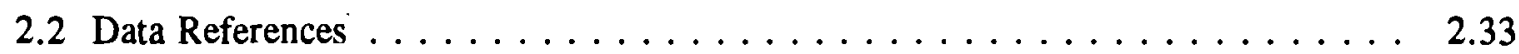


3.0 Sludge Wash Factors Evaluation $\ldots \ldots \ldots \ldots \ldots \ldots \ldots \ldots \ldots \ldots \ldots \ldots \ldots \ldots$

4.0 Spreadsheet Evaluation of the Enhanced Sludge Wash Process $\ldots \ldots \ldots \ldots \ldots \ldots .4 .1$

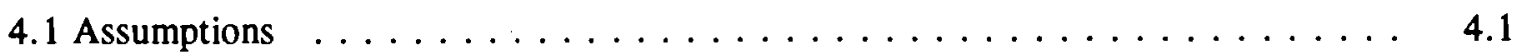

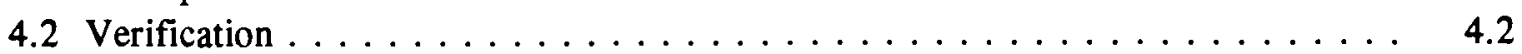

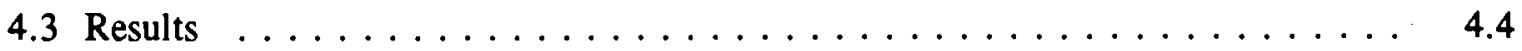

4.3.1 Specific Tank Sludges $\ldots \ldots \ldots \ldots \ldots \ldots \ldots \ldots \ldots \ldots . \ldots \ldots$

4.3.2 Different Waste Types $\ldots \ldots \ldots \ldots \ldots \ldots \ldots \ldots \ldots .4 .4$

Enhanced Sludge Washing Spreadsheet Evaluations

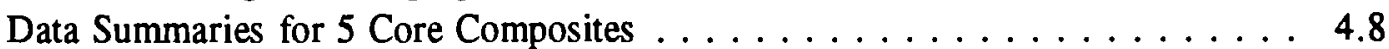

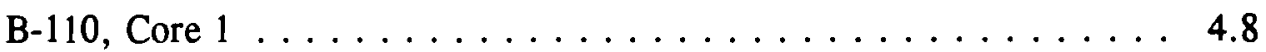

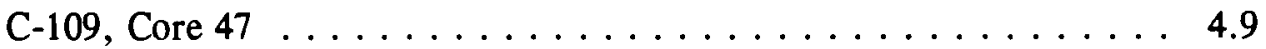

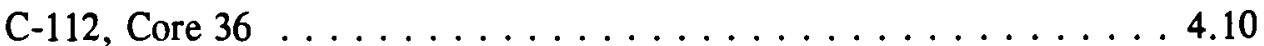

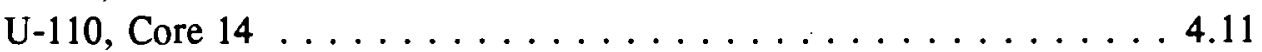

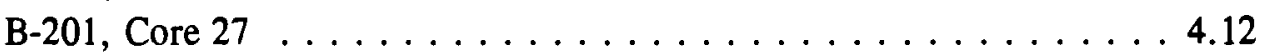

Data Summaries for 20 Specific Tank Sludges $\ldots \ldots \ldots \ldots \ldots \ldots \ldots .13$

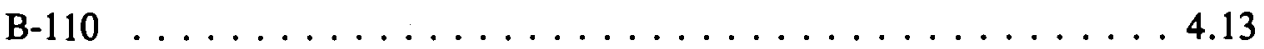

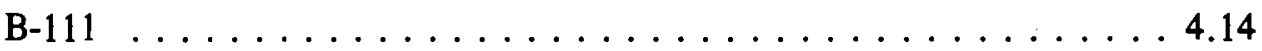

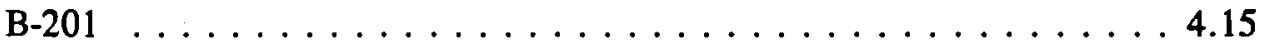

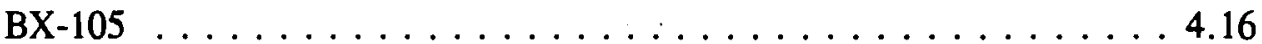

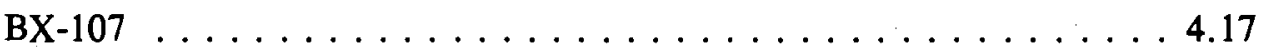

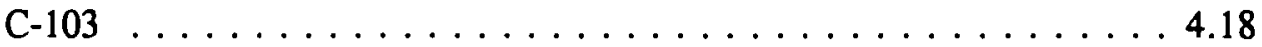

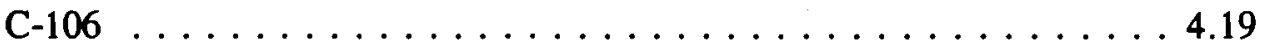

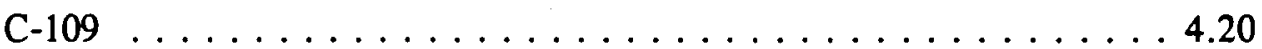

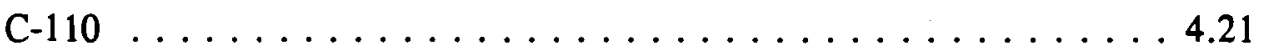

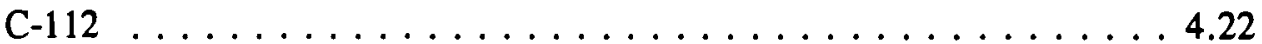

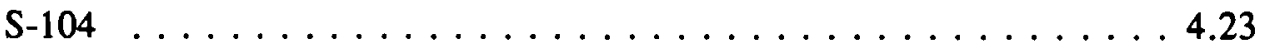

$\mathrm{T}-102 \ldots \ldots \ldots \ldots \ldots \ldots \ldots \ldots \ldots \ldots \ldots . \ldots \ldots .24$

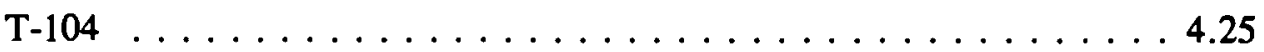

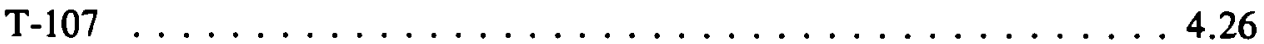

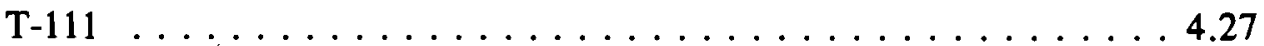

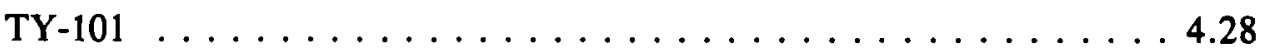

TY-103 . . . . . . . . . . . . . . . . . . . . 4.29

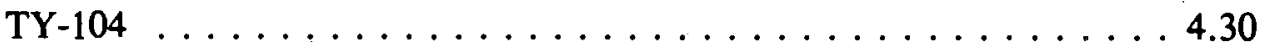

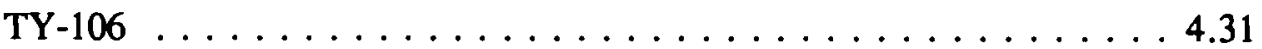

$\mathrm{U}-110 \ldots \ldots \ldots \ldots \ldots \ldots \ldots \ldots \ldots \ldots \ldots \ldots \ldots \ldots \ldots \ldots .32$

Data Summaries for 15 Different Waste Types . . . . . . . . . . . 4.33

Aluminum Cladding Waste $\ldots \ldots \ldots \ldots \ldots \ldots \ldots . \ldots . \ldots \ldots$

Aluminum Cladding Waste - Mix . . . . . . . . . . . 4.34

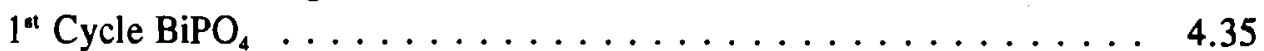

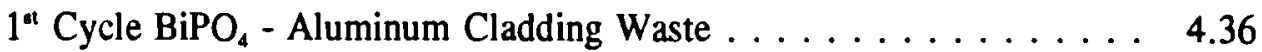


$1^{\text {at }}$ Cycle $\mathrm{BiPO}_{4}$ - Uranium Recovery . . . . . . . . . . . 4.37

$1^{\text {tt }}$ Cycle $\mathrm{BiPO}_{4}$ - Evaporator Bottoms $\ldots \ldots \ldots \ldots \ldots \ldots \ldots$

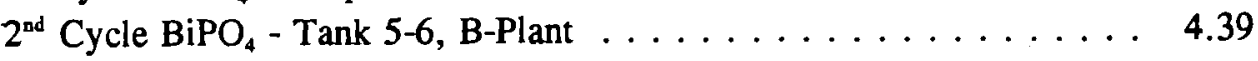

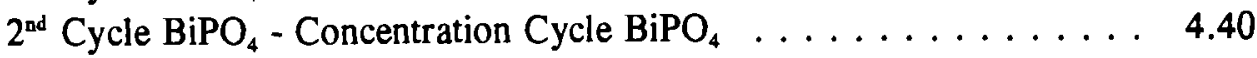

Concentration Cycle $\mathrm{BiPO}_{4} \ldots \ldots \ldots \ldots \ldots \ldots \ldots . \ldots \ldots . \ldots . \ldots . \ldots . \ldots$

PUREX - PUREX Sludge Supernatant . . . . . . . . . . . . . . 4.42

Uranium Recovery - Diatomaceous Earth . . . . . . . . . . . . . 4.43

Uranium Recovery - Cladding Waste - Portland Cement . . . . . . . . 4.44

Uranium Recovery - $1^{\text {st }}$ Cycle $\mathrm{BiPO}_{4}$ (ferrocyanide scavenged) . . . . 4.45

Uranium Recovery (ferrocyanide scavenged) $-1^{\text {al }}$ Cycle $\mathrm{BiPO}_{4} \ldots \ldots 4.46$

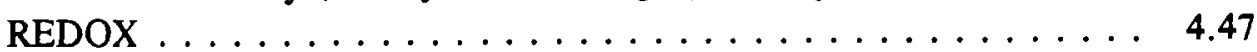

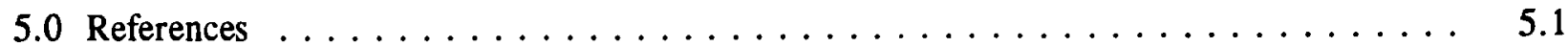

\section{Tables}

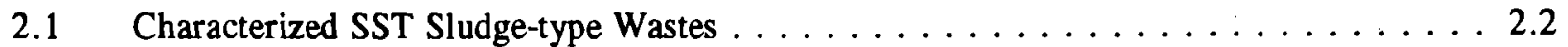

3.1 Calculated Sludge Wash Factors Based on Characterization Data . . . . . . . . . 3.2

3.2 Calculated vs. Assumed Wash Factors (percent soluble in wash) . . . . . . . . . 3.6

4.1 Calculated vs. Experimental Enhanced Sludge Washing Results . . . . . . . . . . . 4.2

4.2 Single-shell Tanks Containing Predominantly Sludge-type Wastes . . . . . . . . . 4.6 
- 


\section{Acknowledgments}

The author wishes to thank the following persons who provided valuable assistance and input to this subtask: A. L. Boldt, M. J. Kupfer, and L. W. Shelton (Systems Engineering), and S. L. Lambert (Vitrification Development) from Westinghouse Hanford Company and J. L. Swanson from Pacific Northwest Laboratory. 



\subsection{Introduction}




\subsection{Introduction}

The Tank Waste Remediation System (TWRS) was established by the U.S. Department of Energy (DOE) to safely manage and dispose of high-level wastes (HLW), transuranic waste, and lowlevel wastes (LLW) stored in underground tanks on the Hanford Site. A key element to the disposal efforts involves pretreatment technologies for separating and/or destroying waste components to reduce waste volumes and to produce waste fractions that are compatible with the final waste forms (glass). Accordingly, work for TWRS to support pretreatment activities is focusing on a strategy to remove radionuclides from aqueous streams to the extent that these streams are suitable for LLW vitrification. TWRS also is focusing on the development of an enhanced sludge washing process to reduce the volume of waste that will require HLW vitrification.

The enhanced sludge washing process involves washing tank sludges with retrieval solutions, i.e., corrosion-inhibited water or dilute caustic, followed by at least one caustic or caustic permanganate wash to remove aluminum, phosphorous, and chromium. Removal of aluminum, a bulk component in some sludges, is expected to reduce HLW glass volumes by reducing the mass of feed to HLW vitrification. Phosphorous and chromium tend to interfere with HLW glass integrity; therefore, removal of these components from the HLW vitrification feed allows higher loading of nonproblematic components and is expected to produce a more homogeneous HLW glass. The enhanced sludge washing process also involves blending wastes to adjust final component concentrations to meet glass feed specifications for acceptable glass formulation.

The effect of this enhanced sludge washing strategy on final waste volumes is evaluated in systems engineering studies by using assumptions about the chemical and physical behavior of the wastes. Chemical assumptions include wash and leach factors, or the fraction of each waste component that will dissolve or will be leached from waste streams during various processing steps. Current evaluations use wash factors and waste stream compositions derived from the overall singleshell tank (SST) and double-shell tank (DST) waste inventories, i.e., idealized blends of SST and DST tank wastes that consist mainly of sodium salts. In actuality, wastes will be blended in batches that could result in larger-than-anticipated HLW volumes. When considering the effect of different blending strategies on HLW volumes, pretreatment scenarios based on specific tank or waste-type inventories also must be evaluated. For that evaluation, wash factors for these tank/waste-type inventories need to be established.

One objective within the Sludge Pretreatment Chemistry Evaluation (Subtask 0503) of the TWRS Tank Waste Treatment Science Task is to establish wash factors for different SST sludges. Efforts so far have focused on compiling selected SST characterization data for review; evaluating current sludge wash factors used in system engineering studies; and developing a spreadsheet to evaluate the effect of enhanced sludge washing on specific tanks sludges. This report presents the work conducted to date and the results derived from the overall efforts. The information is presented as follows: 
Section 2.0, a discussion of the SST data compilation. This section includes characterization data summaries for 27 SST wastes. These summaries, compiled in a format that is easy to use, provide a first step for evaluating tank-specific wash factors needed for the systems engineering studies.

Section 3.0, a discussion of how these SST data were used to evaluate some of the currently assumed sludge wash factors for the overall SST waste inventory and a comparison, for selected elements, of calculated wash factors with assumed system engineering wash factors.

Section 4.0, an introduction to the spreadsheet and the assumptions used to provide insight into the effect of enhanced sludge washing on various tank sludges. This section also provides a comparison of the calculated results from the spreadsheet with recently measured experimental sludge washing results. This section includes spreadsheet evaluation summaries for 5 core composites, 20 tankspecific sludges, and 15 sludge groups that represent different waste types. Tank-specific sludge compositions were derived by averaging core composite data; waste-type compositions were derived by mass-weight-averaging tank compositions. 
2.0 SST Data Compilation and Review 


\subsection{SST Data Compilation and Review}

The first step used to establish wash factors for SST sludges involved compiling data from existing tank waste characterization reports. These data were summarized on tank-specific worksheets that show how much of each element went into solution with water and rigorous acid $\left(\mathrm{HNO}_{3}-\mathrm{HCl}\right)$ digestions and how much of each element had to be fused with caustic before going into solution prior to analysis. These worksheets provided a uniform format for reviewing and comparing data, as well as the means to verify whether the data set for each tank was complete. The characterization data summary worksheets, along with a key to help interpret them, are provided at the end of this section.

Worksheets were completed for the $27^{(a)}$ SSTs listed in Table 2.1. This table also provides, for each tank, the volume of waste; the percentage of solids accounted for with analysis; and the potentially important components that were omitted from analysis. Note that the anions that were omitted from analysis could account for up to 15 to 40 dry weight percent of each waste in Tanks A-102, A-103, A-106, BX-104, C-104, and C-105. The $\mathrm{SO}_{4}{ }^{2-}, \mathrm{Cl}^{-}$, and $\mathrm{F}^{-}$anions are especially important because of their potential impact on HLW vitrification.

(a) Characterization reports were obtained for 29 SSTs; however, data for Tanks T-105 and B-202 were not entered onto spreadsheets. Data for Tank T-105 were not included because these data represented segment analyses instead of core composite analyses. Because of insufficient sample size, no composites were prepared for analysis. No concise data summary tables were obtained for Tank B-202, and data for this tank will be entered onto spreadsheets at a later date. 
Table 2.1. Characterized SST Sludge-type Wastes

\begin{tabular}{|c|c|c|c|}
\hline Tank & $\begin{array}{c}\text { kgal solids } \\
\text { in tank }\end{array}$ & $\begin{array}{c}\text { dry wt\% solids } \\
\text { identified }\end{array}$ & $\begin{array}{c}\text { Components omitted } \\
\text { from analysis }\end{array}$ \\
\hline A-102 & 37 & 86 & $\mathrm{Cl}^{-}, \mathrm{F}^{-}, \mathrm{CO}_{3}{ }^{2}, \mathrm{SO}_{4}{ }^{2-}, \mathrm{NO}_{2}^{-}$ \\
\hline A-103 & 366 & 73 & $\mathrm{Cl}^{-}, \mathrm{F}^{-}, \mathrm{CO}_{3}{ }^{2-}, \mathrm{SO}_{4}{ }^{2-}, \mathrm{NO}_{2}^{-}$ \\
\hline A-106 & 125 & 86 & $\mathrm{Cl}^{-}, \mathrm{F}^{-}, \mathrm{CO}_{3}^{2}, \mathrm{SO}_{4}^{2-}, \mathrm{NO}_{2}^{-}$ \\
\hline B-110 & 245 & 103 & \\
\hline B-111 & 236 & 103 & \\
\hline B-201 & 28 & 111 & \\
\hline BX-104 & 96 & 62 & $\mathrm{Cl}^{-}, \mathrm{F}^{-}, \mathrm{CO}_{3}^{2}, \mathrm{SO}_{4}^{2-}, \mathrm{NO}_{2}^{-}$ \\
\hline BX-105 & 46 & 96 & $\mathrm{Cl}^{-}, \mathrm{F}^{-}, \mathrm{CO}_{3}{ }^{2-}, \mathrm{SO}_{4}^{2-}, \mathrm{NO}_{2}^{-}$ \\
\hline BX-107 & 344 & 105 & \\
\hline $\mathrm{C}-103$ & 62 & 114 & $\mathrm{Cl}^{-}, \mathrm{F}^{\cdot}, \mathrm{CO}_{3}{ }^{2}, \mathrm{SO}_{4}^{2-}, \mathrm{NO}_{2}^{-}$ \\
\hline C-104 & 295 & 83 & $\mathrm{Cl}^{-}, \mathrm{F}, \mathrm{CO}_{3}{ }^{2}, \mathrm{SO}_{4}{ }^{2}, \mathrm{NO}_{2}^{-}$ \\
\hline C-105 & 150 & 57 & $\mathrm{Cl}^{-}, \mathrm{F}^{-}, \mathrm{CO}_{3}^{2}, \mathrm{SO}_{4}^{2-}, \mathrm{NO}_{2}^{-}$ \\
\hline C-106 & 229 & 103 & $\mathrm{Cl}^{-}, \mathrm{F}^{-}, \mathrm{CO}_{3}^{2}, \mathrm{SO}_{4}^{2-}, \mathrm{NO}_{2}^{-}$ \\
\hline$C-109$ & 62 & 93 & $\mathrm{Bi}$ \\
\hline $\mathrm{C}-110$ & 187 & 98 & \\
\hline $\mathrm{C}-112$ & 104 & 107 & $\mathrm{Bi}$ \\
\hline S-104 & 293 & 102 & \\
\hline $\mathrm{T}-102$ & 19 & 99 & \\
\hline $\mathrm{T}-104$ & 442 & 101 & \\
\hline $\mathrm{T}-107$ & 171 & 98 & \\
\hline$T-111$ & 453 & 94 & \\
\hline TY-101 & 118 & 107 & $\mathrm{CO}_{3}^{2-}, \mathrm{NO}_{2}, \mathrm{Ca}, \mathrm{Mg}, \mathrm{K}$ \\
\hline TY-103 & 162 & 101 & $\mathrm{Ca}, \mathrm{Mg}, \mathrm{K}$ \\
\hline TY-104 & 43 & 97 & $\mathrm{Ca}, \mathrm{Mg}, \mathrm{K}$ \\
\hline TY-105 & 231 & 75 & $\mathrm{CO}_{3}^{2-}, \mathrm{NO}_{2} ; \mathrm{SO}_{4}^{2}, \mathrm{Ca}, \mathrm{Mg}, \mathrm{K}$ \\
\hline TY-106 & 17 & 101 & $\mathrm{Ca}, \mathrm{Mg}, \mathrm{K}$ \\
\hline $\mathrm{U}-110$ & 186 & 100 & \\
\hline Total & 4,747 & & \\
\hline
\end{tabular}

(a) Hanlon (1994). 


\subsection{Key for Interpreting Characterization Data Summary Worksheets}

This section provides the key to terms and abbreviations used in the data summary worksheets that follow.

$\begin{array}{lll}\text { Waste Type } & \text { CC } & \text { Complexed Concentrate Waste } \\ \text { CW } & \text { Cladding Waste } \\ \text { DSSF } & \text { Double-shell Slurry Feed } \\ \text { EB } & \text { Evaporator Bottom } \\ \text { Evap } & \text { Evaporator Feed } \\ \text { F } & \text { Ferrocyanide scavenged } \\ \text { HS } & \text { Hot Semi Works } \\ \text { IX } & \text { Ion exchange Waste } \\ \text { NC } & \text { Non-complexed Waste }\end{array}$

$\begin{array}{ll}\text { POS } & \text { Purex Organic Solvent } \\ \text { PSS } & \text { Purex Sludge Supernatant } \\ \text { SRS } & \text { Strontium Recovery Sludge } \\ \text { TBP } & \text { Uranium Recovery } \\ \text { 1C } & \text { 1st Cycle, } \mathrm{BiPO}_{4} \\ \text { 2C } & \text { 2nd Cycle, } \mathrm{BiPO}_{4} \\ \text { 224 } & \text { Concentration Cycle, } \mathrm{BiPO}_{4} \\ \text { 5-6 } & \text { Tank 5-6, B Plant }\end{array}$

Density $(\mathbf{g} / \mathbf{m L}) \quad$ Reported density of sludge. This is an averaged value of the core composites data in the characterization reports.

Volume (L)

Volume of waste in tank reported in Tank Farm Surveillance and Waste Status Summary Report for October 1993, WHCEP-0182-67.

Mass (kg) Mass of waste in tank [density $(\mathrm{kg} / \mathrm{L}) \mathrm{x}$ volume (L)].

Solids $(\mu \mathrm{g} / \mathrm{g}) \quad$ Reported $\mu \mathrm{g}$ solids/g sludge; remaining mass is assumed to be interstitial and hydration waters. This is an averaged value of the core composite data in the characterization reports.

pH

Reported or estimated $\mathrm{pH}$ of sludge.

Oxide Factor

"Oxide Factor" is used to covert an element to the oxide form; this factor is applied to the elements in the acid and fusion leaches so that the amount of dry solids dissolved with these treatments can be estimated.

WATER

Reported $\mu$ g element (or anion) dissolved with $\mu g / g$ sludge (element) water/g sludge sample. This is an averaged value of the core composite data in the characterization reports. Approximately $1 \mathrm{~g}$ sample is contacted with $100 \mathrm{~mL}$ water. Column total: the approximate waste mass (dry solids basis) that is removed with water or that might be removed with a dilute caustic wash. 
WATER

kg/tank

(element)

WATER

\% element dissolved

ACID

$\mu \mathrm{g} / \mathrm{g}$ sludge

(element)

ACID

$\mathrm{kg} / \operatorname{tank}$

(element)

ACID

$\mu \mathrm{g} / \mathrm{g}$ sludge

(oxide)

ACID

\% element dissolved

FUSION

$\mu \mathrm{g} / \mathrm{g}$ sludge

(element)

FUSION

$\mathrm{kg} / \operatorname{tank}$

(element)

FUSION

$\mu \mathrm{g} / \mathrm{g}$ sludge

(oxide)
Estimated elemental mass that might be removed from tank with washing.

$\mu \mathrm{g}$ element (or anion) in water wash $/ \mu \mathrm{g}$ total element in sludge x 100 (also referred to as "wash factor").

Reported $\mu \mathrm{g}$ element dissolved with acid digestion/g sludge. This is an averaged value of the core composites data in the characterization reports. Sample is generally contacted with $\mathrm{HNO}_{3}$ and boiled; contacted again with $\mathrm{HNO}_{3}$ followed by $\mathrm{HCl}$ (boiling); contacted again with $\mathrm{HCl}$. A 100-mg sample might be contacted with a total of $10 \mathrm{~mL}$ acid $\left(5 \mathrm{~mL} \mathrm{HNO}_{3}\right.$ and $\left.5 \mathrm{~mL} \mathrm{HCl}\right)$.

Maximum elemental mass that might be removed from tank with rigorous acid treatment.

Estimated mass of oxide species dissolved with acid/g sludge. Column total: the approximate waste mass (dry solids basis) that is removed with a rigorous acid digestion. In most cases, this would be considered a maximum.

$\mu \mathrm{g}$ element in acid leach $/ \mu \mathrm{g}$ total element in sludge $\mathrm{x} 100$.

Reported $\mu$ g element dissolved with a caustic fusion/g sludge. This is an averaged value of the core composites data in the characterization reports. The sample is treated with $\mathrm{KOH}$; fused at very high temperatures to form a "melt"; and dissolved with water.

Minimum elemental mass that would remain in tank after washing and rigorous acid treatments.

Estimated mass of oxide species dissolved with fusion/g sludge. Column total: the approximate residual waste mass (dry solids basis) that would remain after a water wash followed by an acid dissolution treatment sequence. 
FUSION

$\mu \mathrm{g}$ element in fusion $/ \mu \mathrm{g}$ total element in sludge $\mathrm{x} 100$.

$\%$ element dissolved

TOTAL

$\mu \mathrm{g} / \mathrm{g}$ sludge

Reported total $\mu$ g element (or anion)/g sludge sample.

(element)

End of column percentage: summation of percent solids

dissolved from the water wash, acid leach, and caustic

treatment. This figure should be $100 \pm 10 \%$. If this figure

is less than $90 \%$, chances are that an important

element/elements was not analyzed.

Note: Analytical results are usually valid to three significant figures; therefore, calculated values in the worksheets should be considered significant to three figures only. 


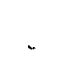

\section{•}


Characterization Data Summary Worksheets 


\begin{tabular}{|c|c|c|c|c|c|c|c|c|c|c|c|c|c|}
\hline $\begin{array}{l}\text { A-102 (DSSF) } \\
\text { Density (g/mL) } \\
\text { Volume (L) } \\
\text { Mass (kg) } \\
\text { Solids (ug/g) } \\
\sim \text { pH } \\
\end{array}$ & $\begin{array}{r}1.590 \\
140045 \\
222672 \\
648500 \\
13.1 \\
\end{array}$ & & & & & & & & & & & & \\
\hline Component & $\begin{array}{l}\text { Oxide } \\
\text { Factor }\end{array}$ & $\begin{array}{r}\text { ug/g sludge } \\
\text { (element) }\end{array}$ & $\begin{array}{r}\text { WATER - } \\
\text { kg/tank } \\
\text { (element) }\end{array}$ & $\begin{array}{c}\text { \% element } \\
\text { dissolved }\end{array}$ & $\begin{array}{r}\text { ug/g sludge } \\
\text { (element) }\end{array}$ & $\begin{array}{r}\mathrm{kg} / \text { tank ug/g sludge } \\
\text { (element) }\end{array}$ & $\begin{array}{c}\% \text { element } \\
\text { dissolved }\end{array}$ & $\begin{array}{r}\text { ug/g sludge } \\
\text { (element) }\end{array}$ & $\begin{array}{r}\mathrm{kg} / \operatorname{tank} \\
\text { (element) }\end{array}$ & $\begin{array}{l}\text { ug/g sludge } \\
\text { (oxide) }\end{array}$ & $\begin{array}{c}\text { \% element } \\
\text { dissolved }\end{array}$ & $\begin{array}{c}\text { ug/g sludge } \\
\text { (element) }\end{array}$ & $\begin{array}{r}\overline{\mathrm{kg} / \operatorname{tank}} \\
\text { (element) }\end{array}$ \\
\hline Aluminum & 1.889 & 11950.000 & 2660.9 & 51.36 & NA & & & 11315.000 & 2519.5 & 21374.035 & 48.64 & 23265.000 & 5180.5 \\
\hline Barium & 1.117 & 3.085 & 0.7 & 0.35 & NA & & & 879.000 & 195.7 & 981.843 & 99.65 & 882.085 & 196.4 \\
\hline Bismuth & 1.115 & 67900 & 15.1 & 3.91 & NA & 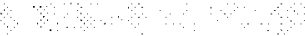 & & 1670.000 & 371.9 & 1862.050 & 96.09 & 1737.900 & 387.0 \\
\hline Boron & 3.220 & 14.200 & 3.2 & 100.00 & NA & & & 0.000 & 0.0 & 0.000 & 0.00 & 14.200 & 3.2 \\
\hline Cadmium & 1.142 & 15.400 & 3.4 & 23.75 & NA & & & 49.450 & 11.0 & 56.472 & 76.25 & 64.850 & 14.4 \\
\hline Calcium & 1.399 & 86.600 & 19.3 & 3.34 & NA & & & 2505.000 & 557.8 & 3504.495 & 96.66 & 2591.600 & 577.1 \\
\hline Chromium & 1.462 & 130.000 & 28.9 & 2.24 & NA & & & 5665.000 & 1261.4 & 8282.230 & 97.76 & 5795.000 & 1290.4 \\
\hline Cobalt & 1.407 & 6.950 & 1.5 & 25.14 & NA & & & 20.700 & 4.6 & 29.125 & 74.86 & 27.650 & 6.2 \\
\hline Copper & 1.252 & 2.320 & 0.5 & 2.79 & NA & & & 80.800 & 18.0 & 101.162 & 97.21 & 83.120 & 18.5 \\
\hline Iron & 1.430 & 5.555 & 1.2 & 0.04 & NA & & & 13930.000 & 3101.8 & 19919.900 & 99.96 & 13935.555 & 3103.1 \\
\hline Lead & 1.077 & 50.000 & 111 & 4.22 & NA & & & 1135.500 & 252.8 & 1222.934 & 95.78 & 1185.500 & 264.0 \\
\hline Magnesium & 1.658 & 1.565 & 0.3 & 0.11 & NA & & & 1380.000 & 307.3 & 2288.040 & 99.89 & 1381.565 & 307.6 \\
\hline Manganese & 1.582 & 123.200 & 27.4 & 5.73 & NA & & & 2027.500 & 451.5 & 3207.505 & 94.27 & 2150.700 & 478.9 \\
\hline Nickel & 1.409 & 14.500 & 3.2 & 2.76 & NA & & & 511.500 & 113.9 & 720.704 & 97.24 & 526.000 & 117.1 \\
\hline Phosphate & 1.000 & 13380.000 & 2979.3 & 85.15 & NA & & & 2334.000 & 519.7 & 2334.000 & 14.85 & 15714.000 & 3499.1 \\
\hline Potassium & 1.000 & 2435.000 & 542.2 & 86.49 & NA & & & 380.500 & 84.7 & 380.500 & 13.51 & 2815.500 & 626.9 \\
\hline Silicon & 2.139 & 129.500 & 28.8 & 0.78 & NA & & & 16400.000 & 3651.8 & 35079.600 & 99.22 & 16529.500 & 3680.6 \\
\hline Silver & 1.074 & 6.170 & 1.4 & 2.50 & NA & & & 241.000 & 53.7 & 258.834 & 97.50 & 247.170 & 55.0 \\
\hline Sodium & 1.000 & 172500.000 & 38410.8 & 92.22 & NA & & & 14545.000 & 3238.8 & 14545.000 & 7.78 & 187045.000 & 41649.6 \\
\hline Strontium & 1.183 & 0.386 & 0.1 & 0.40 & NA & & & 97.200 & 21.6 & 114.988 & 99.60 & 97.586 & 21.7 \\
\hline Zine & 1245 & NR & NR & & NA & & & NR & NR & & & NR & NR \\
\hline Zirconium & 1.351 & 37.200 & 8.3 & 2.58 & NA & & & 1402.000 & 312.2 & 1894.102 & 97.42 & 1439.200 & 320.5 \\
\hline Uranlum & 1.202 & 5715 & 1.3 & 0.06 & NA & & & 9535.000 & 2123.2 & 11461.070 & 99.94 & 9540.715 & 2124.4 \\
\hline Nitrate & 1.000 & 178500.000 & 39746.9 & $\leq 100.00$ & NA & & & NR & NR & & & $\geq 178500.000$ & 39746.9 \\
\hline Chloride & 1,000 & NR & NR & & NA & & & NR & NR & & & NR & NR \\
\hline Fluoride & 1.000 & NR & NR & & NA & & & NR & NR & & & NR & NR \\
\hline Hydroxide (1) & 1.000 & 30100,000 & 6702.4 & $\leq 100.00$ & NA & & & NR & NR & & & $\geq 30100.000$ & 6702.4 \\
\hline Carbonate & 1.000 & NR & NR & & NA & & & NR & NR & & & NR & \\
\hline TOC & 2.450 & 18546.500 & 1685.6 & $\leq 100.00$ & NA & & & NR & NR & & & $\geq 7570.000$ & 1685.6 \\
\hline Nitrite & 1.000 & NR & NR & & NA & & & NR & NR & & & NR & NR \\
\hline Sulfate & 1.000 & NR & NR & & $\mathrm{NA}$ & & & NR & NR & & & NR & NR \\
\hline Total & & $\begin{array}{r}428111.746 \\
66.02 \\
\end{array}$ & total solids & & & $\begin{array}{r}0.000 \\
0.00\end{array}$ & $\begin{array}{l}\text { \%total solids } \\
\text { in acid }\end{array}$ & & & $\begin{array}{r}129618.587 \\
19.99\end{array}$ & \%total solids & 86.00 & 6 mass \\
\hline Radionuclides & & uCi/g & Ci/tank & 6 dissolved & $\mathrm{uCi} / \mathrm{g}$ & Ci/tank & $\%$ dissolved & uCi/g & Ci/tank & & $\%$ dissolved & uCi/g & Ci/tank \\
\hline $\mathrm{Pu} 239,240$ & & 0.067 & 14.9 & 3.33 & NA & & & 1.935 & 430.9 & & 96.67 & 2.002 & 445.7 \\
\hline C- 14 & & 0.001 & 0.3 & $\leq 100.00$ & NA & & & 0.000 & 0.0 & & 0.00 & $\geq 0.001$ & 0.3 \\
\hline Sr-90 & & 0.843 & 187.6 & 0.14 & NA & & & 603.500 & 1343823 & & 99.86 & $604: 343$ & 134569.9 \\
\hline Tc-99 & & 0.100 & 22.3 & $\leq 100.00$ & NA & & & 0.000 & 0.0 & & 0.00 & 0.100 & 22.3 \\
\hline$A m-241$ & & 0.003 & 0.6 & 0.21 & NA & & & 1.210 & 269.3 & & 99.79 & 1.212 & 269.9 \\
\hline$C 0-60$ & & 0.002 & 0.5 & 0.29 & NA & & & 0.824 & 183.5 & & 99.71 & 0.826 & 184.0 \\
\hline Cs-137 & & 96.950 & 21588.0 & 69.50 & NA & & & 42.550 & 9474.7 & & 30.50 & 139.500 & 31062.7 \\
\hline $1-129$ & & 0.000039 & 0.01 & $\leq 100.00$ & NA & & & NR & NR & & & $\geq 0.000039$ & 0.01 \\
\hline
\end{tabular}

$1-129$

NR

NR

$\geq 0.000039$

1. Hydroxide estimated based on Al concentration.

2. TOC on water leach only

3. Nitrate on water leach only, would not account for any nitrate associated with cancrinite

4. Reported value of $3.9 \mathrm{e}-5 \mathrm{uCl} / \mathrm{g}$ for 1 is direct report value for total sample
NR - Not Reported

NA - No Analysis Performed

$<D L$ - Less than Detection Limit 


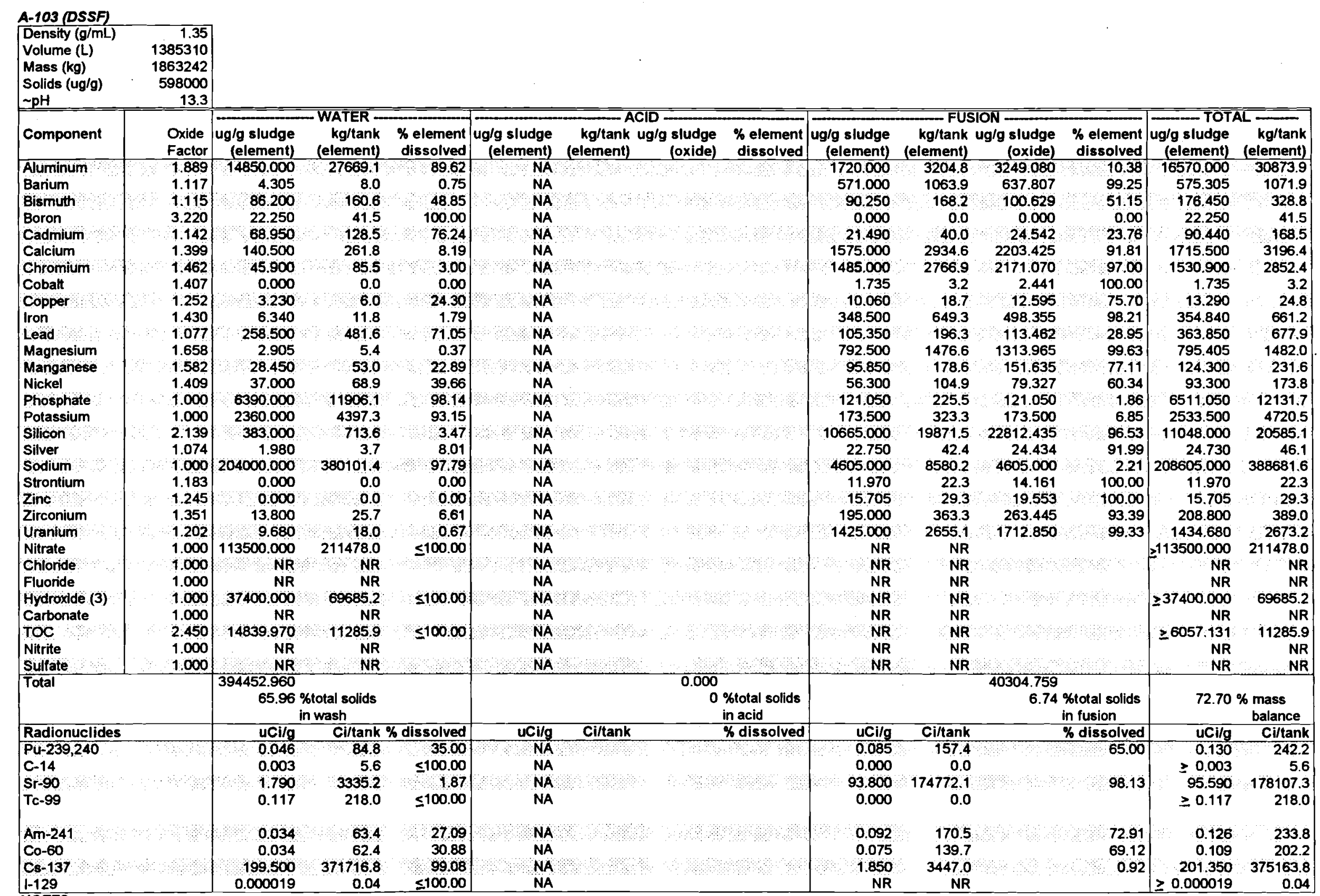

NOTTES:

1. Nitrate on water leach only, would not account for any nitrate associated with cancrinite

2. TOC on water leach only

3. Hydroxide estimated based on Al concentration.

4. Reported value of $1.9 e-5 \mathrm{uCi} g$ for I is direct report value for total sample

NR - Not Reported

NA - No Analysis Performed

$<D L$ - Less than Detection Limit 

A-106 (CC, NC, EB, B-Plant) $\begin{array}{lr}\text { Density }(\mathrm{g} / \mathrm{mL}) & \mathbf{1} \\ \text { Volume (L) } & 473125 \\ \text { Vass }(\mathrm{kg}) & 733344\end{array}$ $\begin{array}{ll}\text { Mass (kg) } & 733344 \\ \text { Solids (ug/g) } & 559500\end{array}$ \begin{tabular}{lr}
$\begin{array}{l}\text { Solids (ug/g) } \\
\sim \mathrm{pH}\end{array}$ \\
\hline
\end{tabular}

\begin{tabular}{|l|l|l|}
\hline \hline Component & $\begin{array}{l}\text { Oxide } \\
\text { Factor }\end{array}$ & $\begin{array}{c}\text { ug/g } \\
\text { (ele }\end{array}$ \\
\hline Aluminum & 1.889 & 1031 \\
\hline
\end{tabular}
ug/g sludge WATER $\mathrm{kg} /$ tank \% element ug/g sludge $\mathrm{kg} /$ tank $\mathrm{Ag} / \mathrm{g}$ $\mathrm{kg} /$ tank \% element ug/g sludge $\mathrm{kg} / \mathrm{tank}$ ug/g Aluminum Barium Bismuth Boron

Cadmium

Calcium

Chromium

Cobalt

Copper

Iron

Lead

Magnesium

Manganese

Nickel

Phosphate

Potassium

Silicon

Sitver

Sodium

Strontium

Zinc.

Zirconium

Uranium

Nitrate

Chloride

Fluoride
Hydroxide (2)

Carbonate

Toc

Nitrite
Sulfate

Total

1.889 10310.000

(element) dissolved (element) (element)

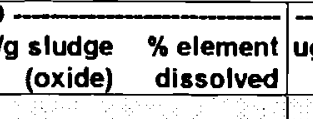

ug/g sludge
(element) FUSION -

g/tank ug/g sludge \% element ug/g sludge $\mathrm{kg} / \mathrm{tank}$ 1.117
1.115
3.220

2.260
49.650

\begin{tabular}{rr|r}
560.8 & 45.24 \\
1.7 & 0.11
\end{tabular}

NA
NA
NA

18.800

$\begin{array}{rr}1.7 & 0.11 \\ 36.4 & 21.34\end{array}$ $\begin{array}{rr}0.11 & \text { NA } \\ 21.34 & \text { NA }\end{array}$

11.250

$\begin{array}{rr}13.8 & 100.00 \\ 8.3 & 29.22 \\ 47.2 & 1.22\end{array}$

64.350
413.000

8.3

29.22 NA

$413.000 \quad 302.9$

1.22 NA

4.060

8.16 NA

4.350

3.0

$30.10 \quad$ NA

36.550

\begin{tabular}{r|rrr|r}
1.658 & 0.607 & 0.4 & 0.02 & NA \\
1.582 & 90.250 & 66.2 & 6.00 & NA \\
1.409 & 12.250 & 9.0 & 1.96 & NA \\
1.000 & 49800.000 & 38520.5 & 94.78 & NA
\end{tabular}

\begin{tabular}{r|rrr|r}
1.658 & 0.607 & 0.4 & 0.02 & NA \\
1.582 & 90.250 & 66.2 & 6.00 & NA \\
1.409 & 12.250 & 9.0 & 1.96 & NA
\end{tabular}

3.2

$5.20 \quad$ NA

26.8

0.02
3.39

1.409

38520.5

$\begin{array}{rr}1.96 & \text { NA } \\ 94.78 & \text { NA } \\ 75.45 & \text { NA } \\ 0.20 & \text { NA }\end{array}$

\begin{tabular}{r|rrr|r}
1.000 & 1695.000 & 1243.0 & 75.45 & NA \\
2.139 & 76.050 & 55.8 & 0.20 & NA
\end{tabular}

\begin{tabular}{r|rr}
1.074 & 4.515 & 3.3 \\
1.000 & 102150.000 & 74911.1
\end{tabular}

$0.20 \quad$ NA

0.449
1.850

\begin{tabular}{r|r}
1.351 & 20.800 \\
1.202 & 8.885
\end{tabular}

0.3
1.4

1.60
83.87

0.86 NA

\begin{tabular}{rr|r}
15.3 & 100.00 & 1.70
\end{tabular}

$6.5 \quad 1.70 \quad$ NA

$\begin{array}{rrr}78100.000 & 57274.1 & \leq 100.00\end{array}$ NA

$\begin{array}{rrr}\text { NR } & \text { NR } & \text { NA } \\ 26000.000 & 19066.9 & \leq 100.00\end{array}$

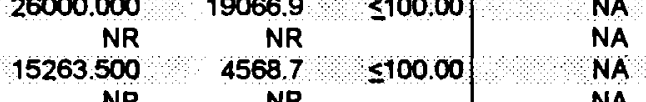

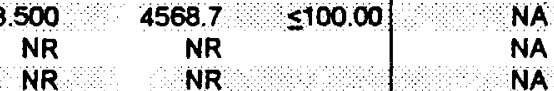

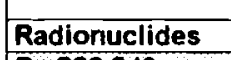

284142.486

79 \%total solids

in wash
Ci/tank \% dissolved $\quad$ uCi/g C Ci/tank

Pu 239,240

C-14

Sr-90

Tc-99

Am-241

Co-60

Cs-137

1-129

uCi/g
12480.000 lement) \begin{tabular}{rrr} 
lved & (element) & (element) \\
\hline 4.76 & 22790.000 & 16712.9
\end{tabular}

\begin{tabular}{rrrr|rr}
183.000 & 134.2 & 204.045 & 78.66 & 232.650 & 170.6 \\
0.000 & 0.0 & 0.000 & 0.00 & 18.800 & 13.8
\end{tabular}

\begin{tabular}{rrrr|rr|}
27.250 & 20.0 & 0.000 & 0.00 & 18.800 & 13.8 \\
& 20 & 31.120 & 70.78 & 38.500 & 28.2
\end{tabular}

\begin{tabular}{|l|l|l|l|r|r|}
5205.000 & 3817.1 & 7281.795 & 98.78 & 5269.350 & 3864.2 \\
\hline 4650.000 & 3410.0 & 6798.300 & 91.84 & 5063.000 & 3712.9 \\
\hline
\end{tabular}

\begin{tabular}{ll|rr|}
13.368 & 91.84 & 5063.000 & 3712.9
\end{tabular}

\begin{tabular}{rrrrrr}
79.300 & 582 & 99.284 & 94.80 & 83.650 & 9.9 \\
\hline
\end{tabular}

\begin{tabular}{|rrrr|rr|r|}
26350.000 & 19323.6 & 37680.500 & 99.98 & 26354.060 & 19326.6 \\
\hline
\end{tabular}

\begin{tabular}{llll|r|r|}
1042.500 & 764.5 & 1122.773 & 96.61 & 1079.050 & 791.3 \\
\hline
\end{tabular}

\begin{tabular}{|lllll|ll|}
2835.000 & 2079.0 & 4700.430 & 99.98 & 2835.607 & 2079.5 \\
\hline
\end{tabular}

\begin{tabular}{rrrrr|rr|}
1413.500 & 1036.6 & 2236.157 & 94.00 & 1503.750 & 1102.8 \\
612.000 & 448.8 & 862.308 & 98.04 & 624.250 & 457.8 \\
\hline
\end{tabular}

\begin{tabular}{|llll|l|l|}
2743.500 & 2011.9 & 2743.500 & 5.22 & 52543.500 & 38532.4
\end{tabular}

\begin{tabular}{|lrrr|rr|}
551.500 & 404.4 & 551.500 & 24.55 & 2246.500 & 1647.5
\end{tabular}

\begin{tabular}{|l|l|l|l|l|l|}
38300.000 & 28087.1 & 81923.700 & 99.80 & 38376.050 & 28142.8 \\
\hline
\end{tabular}

\begin{tabular}{rrrr|r|r|}
277.500 & 203.5 & 298.035 & 98.40 & 282.015 & 206.8 \\
\hline
\end{tabular}

\begin{tabular}{llll|lll}
19650.000 & 14410.2 & 19650.000 & 16.13 & 121800.000 & 89321.3
\end{tabular}

\begin{tabular}{rrrr|rr}
52.000 & 38.1 & 61.516 & 99.14 & 52.449 & 38.5 \\
\hline & 0.000 & 0.000 & 0.00 & 1.850 & 1.4
\end{tabular}

\begin{tabular}{rrrr|rr|}
0.000 & 0.0 & 0.000 & 0.00 & 1.850 & 1.4 \\
1204.000 & 882.9 & 1626.604 & 98.30 & 1224.800 & 898.2
\end{tabular}

\begin{tabular}{llll|rr}
793.000 & 581.5 & 953.186 & 98.89 & 801.885 & 588.1
\end{tabular}

\begin{tabular}{r|l|rr|r} 
NR & NR & $\geq 78100.000$ & 57274.1 \\
NR & NR & NR & NR \\
NR & NR & NR & NR \\
NR & NR & NR & NR & N \\
NR & NR & & $\geq 26000.000$ & 19066.9 \\
& NR & NR
\end{tabular}

\begin{tabular}{ll|rr} 
NR & NR & $\geq 26000.000$ & 19066.9 \\
NR & NR & NR & NR \\
NR & NR & $\geq 6230.000$ & 4568 \\
NR & NR & &
\end{tabular}

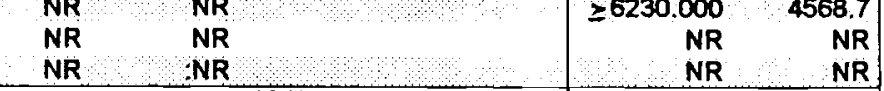

0.000

$0.00 \%$ total solids

in acid

14.290

$34.82 \%$ total solids

in fusion

$85.60 \%$ mass

\begin{tabular}{r|rr} 
& & balance \\
\hline 96.27 & $\mathrm{uCi} / \mathrm{g}$ & Ci/tank \\
\hline
\end{tabular}

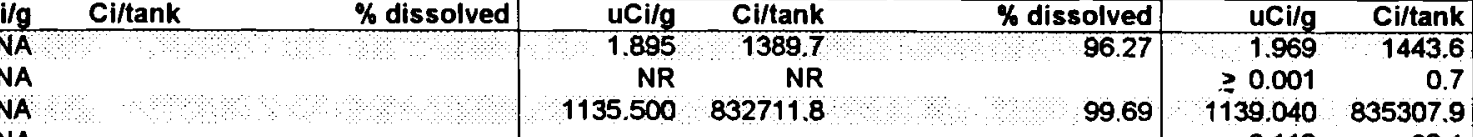

$\begin{array}{rrr}2596.0 & \leq 100.00 & \text { NA } \\ & 0.31 & \text { NA }\end{array}$

\begin{tabular}{rr|r}
2596.0 & 0.31 & NA \\
82.1 & $\leq 100.00$ & NA
\end{tabular}

$\begin{array}{lll}0.112 & 82.1 \leq 100.00\end{array}$

1. Nitrate on water leach only, would not account for

$\begin{array}{lll}2.6 & 0.43 & 0\end{array}$

$76817.8 \quad 71.09$

71.09
$\leq 100.00$

NA

NA
NA
NA

$0.817 \quad 5991$

$\begin{array}{rr}0.700 & 513.3 \\ 42.600 & 31240.4\end{array}$

99.57

$\geq 0.112$

82.1

on water leach only

4. Reported value of $1.4 e-4 \mathrm{uCl} / \mathrm{g}$ for $\mathrm{l}$ is direct report value for total sample

NR - Not Reported

NA - No Analysis Performed

$<D L$ - Less than Detection Limit 


\begin{tabular}{|c|c|c|c|c|c|c|c|c|c|c|c|c|c|c|}
\hline $\begin{array}{l}\text { Density }(\mathrm{g} / \mathrm{mL}) \\
\text { Volume }(\mathrm{L}) \\
\text { Mass (kg) } \\
\text { Solids (ug/g) } \\
\sim \mathrm{pH}\end{array}$ & $\begin{array}{r}1.35 \\
927325 \\
1251889 \\
430000 \\
10\end{array}$ & & & & & & & & & & & & & \\
\hline Component & $\begin{array}{l}\text { Oxide } \\
\text { Factor }\end{array}$ & $\begin{array}{r}\text { ug/g sludge } \\
\text { (element) }\end{array}$ & $\begin{array}{r}\text { WATER - } \\
\text { kg/tank } \\
\text { (element) }\end{array}$ & $\begin{array}{c}\% \text { element } \\
\text { dissolved }\end{array}$ & $\begin{array}{r}\text { ug/g sludge } \\
\text { (element) }\end{array}$ & $\begin{array}{r}\mathrm{kg} / \operatorname{tank} \\
\text { (element) }\end{array}$ & $\begin{array}{r}\text { ug/g sludge } \\
\text { (oxide) }\end{array}$ & $\begin{array}{c}\text { \% element } \\
\text { dissolved }\end{array}$ & $\begin{array}{l}\text { ug/g sludge } \\
\text { (element) }\end{array}$ & $\begin{array}{r}\text { kg/tank } \\
\text { (element) }\end{array}$ & $\begin{array}{r}\text { ug/g sludge } \\
\text { (oxide) }\end{array}$ & $\begin{array}{l}\text { \% element } \\
\text { dissolved }\end{array}$ & $\begin{array}{c}\text { ug/g sludge } \\
\text { (element) }\end{array}$ & $\begin{array}{l}\overline{\mathrm{kg} / \tan k} \\
\text { (element) }\end{array}$ \\
\hline Aluminum & 1.889 & $\angle D L$ & & & 1226.000 & 1534.8 & 2315.914 & 100.00 & 0.000 & 0.0 & 0.000 & 0.00 & 1226.000 & 1534.8 \\
\hline Barium & 4.117 & $<D L$ & & & 20.250 & 25.4 & 22.619 & 82.23 & 4.375 & 5.5 & 4.887 & 17.77 & 24.625 & 30.8 \\
\hline Bismuth & 1.115 & 42.750 & 53.5 & 0.21 & 19921.500 & 24939.5 & 22212.473 & 9901 & 157.250 & 196.9 & 175.334 & 0.78 & 20121.500 & 25189.9 \\
\hline Boron & 3.220 & 3.750 & 4.7 & 10.87 & 30.750 & 38.5 & 99.015 & 89.13 & 0.000 & 0.0 & 0.000 & 0.00 & 34.500 & 43.2 \\
\hline Cadmium & 1.142 & 6.250 & 7.8 & 20.83 & 2.500 & 31 & 2855 & 8.33 & 21.250 & 26.6 & 24.268 & 70.83 & 30.000 & 37.6 \\
\hline Calcium & 1.399 & 33.250 & 41.6 & 3.44 & 803.500 & 1005.9 & 1124.097 & 83.09 & 130.250 & 163.1 & 182.220 & 13.47 & 967.000 & 1210.6 \\
\hline Chromium: & 1.462 & 70.500 & 88.3 & 7.25 & 896.000 & 1121.7 & 1309.952 & 92.18 & 5.500 & 6.9 & 8.041 & 0.57 & 972.000 & 1216.8 \\
\hline Cobalt & 1.407 & $<D L$ & & & $<D L$ & 0.0 & 0.000 & ERR & $<D L$ & & & & $<D L$ & \\
\hline Copper & 1.252 & 28.000 & 35.1 & 47.28 & 17.500 & 21.9 & 21.910 & 29.54 & 13.750 & 17.2 & 17.215 & 23.21 & 59.250 & 74.2 \\
\hline Iron & 1.430 & 118.750 & 148.7 & 0.61 & 19084.000 & 23891.0 & 27290.120 & 98.80 & 112.750 & 141.2 & 161.233 & 0.58 & 19315.500 & 24180.9 \\
\hline Lead & 1.077 & $\angle D L$ & & & 649.250 & 812.8 & 699.242 & 98.80 & 7.875 & 9.9 & 8.481 & 1.20 & 657.125 & 822.6 \\
\hline Magnesium & 1.658 & 3.750 & 4.7 & 1.96 & 175.250 & 219.4 & 290.565 & 91.63 & 12.250 & 15.3 & 20.311 & 6.41 & 491.250 & 239.4 \\
\hline Manganese & 1.582 & $<D L$ & & & 70.750 & 88.6 & 111.927 & 78.39 & 19.500 & 24.4 & 30.849 & 21.61 & 90.250 & 113.0 \\
\hline Nickel & 1.409 & $<D L$ & & & 88.500 & 110.8 & 124.697 & 100.00 & NA & & & & 88.500 & 110.8 \\
\hline Phosphate & 1.000 & 23460.000 & 29369.3 & 44.90 & 26961.750 & 33753.1 & 26961750 & 51.60 & 1833.000 & 2294.7 & 1833.000 & 3.51 & 52254.750 & 65417.1 \\
\hline Potassium & 1.000 & 309.250 & 387.1 & 66.22 & 157.750 & 197.5 & 157.750 & 33.78 & NA & & & & 467.000 & 584.6 \\
\hline Silicon & 2.139 & 376.500 & 471.3 & 3.82 & 312.750 & 391.5 & 668.972 & 3.17 & 9165.500 & 11474.2 & 19605.005 & 93.01 & 9854.750 & 12337.1 \\
\hline Silver & 1.074 & 7.750 & 9.7 & 8.93 & 35.250 & 44.1 & 37.859 & 40.63 & 43.750 & 54.8 & 46.988 & 50.43 & 86.750 & 108.6 \\
\hline Sodium & 1.000 & 90836.500 & 113717.2 & 90.71 & 9062.750 & 11345.6 & 9062.750 & 9,05 & 242.250 & 303.3 & 242.250 & 0.24 & 100141.500 & 125366.0 \\
\hline Strontium & 1.183 & $<D L$ & & & 216.750 & 271.3 & 256.415 & 100.00 & 0.000 & 0.0 & 0.000 & 0.00 & 216.750 & 271.3 \\
\hline Zine & 1245 & 22.500 & 28.2 & 4.47 & 65.750 & 82.3 & 81.859 & 13.07 & 415.000 & 519.5 & 516.675 & 82.46 & 503.250 & 630.0 \\
\hline Zirconium & 1.351 & $<D L$ & & & 3.500 & 4.4 & 4.729 & 100.00 & $<D L$ & & & & 3.500 & 4.4 \\
\hline Uranium & 1.202 & $<D L$ & & & 404.000 & 505.8 & 485.608 & 100.00 & NR & NR & & & 404.000 & 505.8 \\
\hline Nitrate & 1.000 & 181125.000 & 226748.3 & $\leq 100.00$ & NR & NR & & & NR & NR & & & $>181125.000$ & 226748.3 \\
\hline Chloride & 1.000 & 1152.500 & 1442.8 & $\leq 100.00$ & NR & NR & & & NR & NR & & & $\geq 1152.500$ & 1442.8 \\
\hline Fluoride & 1.000 & 1781.250 & 2229.9 & $\leq 100.00$ & NR & NR & & & NR & NR & & & $\geq 1781.250$ & 2229.9 \\
\hline Free OH & 1.000 & NR & NR & & NR & NR & & & NR & NR & & & NR & \\
\hline Carbonate & 1.000 & 5054.250 & 6327.4 & $\leq 100.00$ & NR & NR & & & NR & NR & & & $\geq 5054.250$ & 6327.4 \\
\hline TOC & 2.450 & 918.750 & 469.5 & $\$ 100.00$ & NR & NR & & & NR & NR & & & $\simeq 375.000$ & 469.5 \\
\hline Nitrite & 1.000 & 10429.500 & 13056.6 & $\leq 100.00$ & NR & NR & & & NR & NR & & & $\geq 10429.500$ & 13056.6 \\
\hline Sulfate & 1.000 & 11250.000 & 14083.7 & $\leq 10000$ & NR & NR & & & NR & NR & & & $\geq 11250.000$ & 14083.7 \\
\hline Total & & $\begin{array}{r}327030.750 \\
76.05\end{array}$ & $\begin{array}{l}\text { total solids } \\
\text { wash }\end{array}$ & & & & $\begin{array}{r}93343.075 \\
21.71\end{array}$ & $\begin{array}{l}\text { \%total solids } \\
\text { in acid }\end{array}$ & & & $\begin{array}{r}22876.754 \\
5.32\end{array}$ & $\begin{array}{l}\text { \%total solids } \\
\text { in fusion }\end{array}$ & 103.08 & $\begin{array}{l}\% \text { mass } \\
\text { balance }\end{array}$ \\
\hline Radionuclides & & uCi/g & Ci/tank & Th dissolved & uCi/g & Ci/tank & & \% dissolved & $\mathrm{uCi} / \mathrm{g}$ & Ci/tank & & $\%$ dissolved & $\mathbf{u C i} / \mathrm{g}$ & Ci/tank \\
\hline Pu-239,240 & & 0.002 & 1.9 & 1.30 & NA & & & & 0.114 & 142.7 & & 98.70 & 0.116 & 144.6 \\
\hline C-14 & & 0.002 & 2.5 & $\leq 100.00$ & NA & & & & NR & NR & & & $\geq 0.002$ & 2.5 \\
\hline $5 r .90$ & & 0.170 & 2122 & 0.10 & NA & & & & 168.333 & 210734.6 & & 99.90 & 168.503 & 210946.8 \\
\hline Tc-99 & & 0.019 & 23.8 & 93.44 & NA & & & & 0.001 & 1.7 & & 6.56 & 0.020 & 25.5 \\
\hline$A m=241$ & 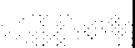 & 0.003 & 3.6 & 3.48 & NA & & & & 0.079 & 98.9 & & 96.52 & 0.082 & 102.5 \\
\hline $\mathrm{Co}-60$ & & 0.000 & 0.5 & $\leqslant 100.00$ & NA & & & & NR & NR & & & $\geq 0.000$ & 0.5 \\
\hline Cs-137 & & 8.589 & 107522 & 56.93 & NA & & & & $\begin{array}{r}6.498 \\
\end{array}$ & 8134.1 & & 43.07 & 15.086 & 18886.3 \\
\hline $\mid-129$ & & $<1 e-6$ & $<0.001$ & & NA & & & & 0.000016 & $<0.02$ & & & $<0.000016$ & $<0.02$ \\
\hline
\end{tabular}

1. lodine value less than 


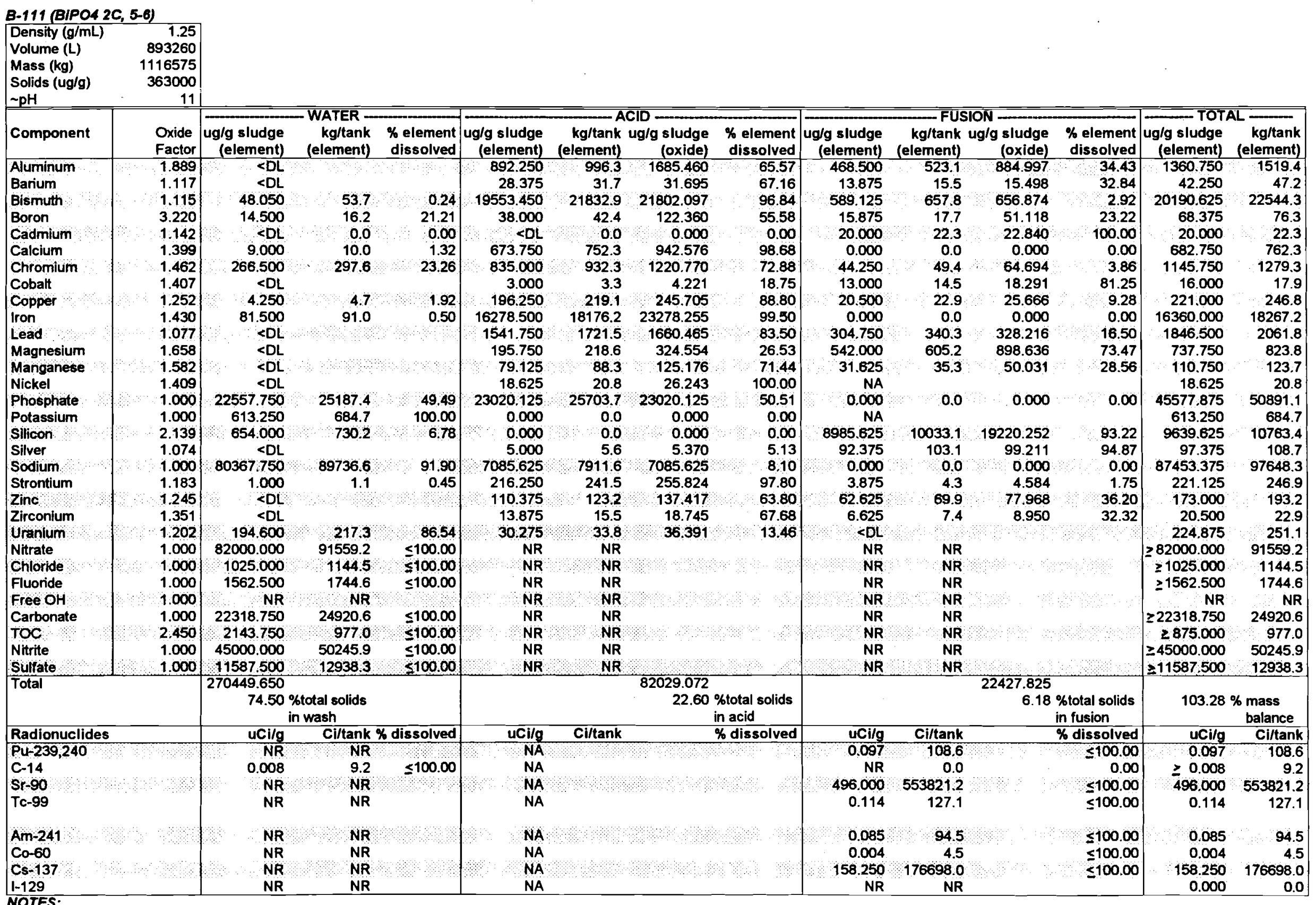

1. Radionuclide analyses on fused sample only, with exception of $\mathrm{C}-14$

2. Co-60 less than value 


\begin{tabular}{|c|c|c|c|c|c|c|c|c|c|c|c|c|c|c|}
\hline $\begin{array}{l}\text { Density (g/mL) } \\
\text { Volume (L) } \\
\text { Mass (kg) } \\
\text { Solids (ug/g) } \\
\sim \mathrm{pH} \\
\end{array}$ & $\begin{array}{r}1.25 \\
105980 \\
132475 \\
396000 \\
11.000 \\
\end{array}$ & & & \multirow[b]{2}{*}{ ug/g sludge } & & \multirow[b]{2}{*}{$\begin{array}{l}\text { \% element } \\
\text { dissolved }\end{array}$} & \multirow[b]{2}{*}{$\begin{array}{r}\text { ug/g sludge } \\
\text { (element) }\end{array}$} & \multirow{2}{*}{\multicolumn{2}{|c|}{ FUSION - }} & \multirow[b]{2}{*}{$\begin{array}{c}\text { \% element } \\
\text { dissolved }\end{array}$} & \multirow[b]{2}{*}{$\begin{array}{l}\text { ug/g sludge } \\
\text { (element) }\end{array}$} & \multirow[b]{2}{*}{$\begin{array}{l}\text { kg/tank } \\
\text { (element) }\end{array}$} \\
\hline Component & $\begin{array}{l}\text { Oxide } \\
\text { Factor }\end{array}$ & $\begin{array}{r}\text { ug/g sludge } \\
\text { (element) }\end{array}$ & $\begin{array}{l}\text { WATER - } \\
\text { kg/tank } \\
\text { (element) }\end{array}$ & $\begin{array}{l}\text { \% element } \\
\text { dissolved }\end{array}$ & & $\begin{array}{r}\mathrm{kg} / \operatorname{tank} \mathrm{A} \\
\text { (element) }\end{array}$ & $\begin{array}{l}\text { CID } \\
\text { ug/g sludge } \\
\text { (oxide) }\end{array}$ & & & & & & & \\
\hline Aluminium & 1.889 & 50.300 & 67 & 1.04 & 3970.000 & 525.9 & 7499.330 & 8178 & 834.000 & 110.5 & 1575.426 & 1718 & 4854,300 & 643.1 \\
\hline Barium & 1.117 & 0.920 & 0.1 & 0.23 & 75.300 & 10.0 & 84.110 & 19.00 & 320.000 & 42.4 & 357.440 & 80.76 & 396.220 & 52.5 \\
\hline Bismuth & 1.115 & 12.400 & 16 & 0.01 & 103400.000 & 13697.9 & 115291.000 & 99.99 & 0.000 & 0.0 & 0.000 & 0.00 & 103412.400 & 13699.6 \\
\hline Boron & 3.220 & 4.860 & 0.6 & 7.48 & 60.100 & 8.0 & 193.522 & 92.52 & 0.000 & 0.0 & 0.000 & 0.00 & 64.960 & 8.6 \\
\hline Cadmium & 1.142 & 0.460 & 0.1 & 1.17 & 2.520 & 0.3 & 2.878 & 6.38 & 36.500 & 4.8 & 41.683 & 92.45 & 39.480 & 5.2 \\
\hline Calcium & 1.399 & 38.700 & 5.1 & 0.28 & 13730.000 & 1818.9 & 19208.270 & 99.72 & 0.000 & 0.0 & 0.000 & 0.00 & 13768.700 & 1824.0 \\
\hline Chromium & 1.462 & 858.000 & 113.7 & 25.63 & 1870.000 & 247.7 & 2733.940 & 55.85 & 620.000 & 82.1 & 906.440 & 18.52 & 3348.000 & 443.5 \\
\hline Cobalt & 1.407 & 0.920 & 0.1 & 1.35 & 6.380 & 0.8 & 8.977 & 9.35 & 60.900 & 8.1 & 85.686 & 89.30 & 68.200 & 9.0 \\
\hline Copper & 1.252 & 0.460 & 0.1 & 0.39 & 55.000 & 7.3 & 68.860 & 46.27 & 63.400 & 8.4 & 79.377 & 53.34 & 118.860 & 15.7 \\
\hline Iron & 1.430 & 5.930 & 0.8 & 0.04 & 15100.000 & 2000.4 & 21593.000 & 99.96 & 0.000 & 0.0 & 0.000 & 0.00 & 15105.930 & 2001.2 \\
\hline Lead & 1.077 & 5.520 & 0.7 & 0.37 & 1080.000 & 143.1 & 1163.160 & 71.50 & 425.000 & 56.3 & 457.725 & 28.14 & 1510.520 & 200.1 \\
\hline Magnesium & 1.658 & 12.900 & 1.7 & 0.38 & 1700.000 & 225.2 & 2818.600 & 49.81 & 1700.000 & 225.2 & 2818.600 & 49.81 & 3412.900 & 452.1 \\
\hline Manganese & 1.582 & 3.450 & 0.5 & 0.01 & 18380.000 & 2434.9 & 29077.160 & 79.62 & 4700.000 & 622.6 & 7435.400 & 20.36 & 23083.450 & 3058.0 \\
\hline Nickel & 1.409 & 3.070 & 0.4 & 0.41 & 746.000 & 98.8 & 1051.114 & 99.59 & NA & & & & 749.070 & 99.2 \\
\hline Phosphate & 1.000 & 1287.000 & 170.5 & 7.08 & 16890.000 & 2237.5 & 16890.000 & 92.92 & 0.000 & 0.0 & 0.000 & 0.00 & 18177.000 & 2408.0 \\
\hline Potassium & 1.000 & 4550.000 & 602.8 & 75.96 & 1440.000 & 190.8 & 1440.000 & 24.04 & NA & & & & 5990.000 & 793.5 \\
\hline Silicon & 2.139 & 662.000 & 87.7 & 2.72 & 1870.000 & 247.7 & 3999.830 & 7.69 & 21790.000 & 2886.6 & 46608.810 & 89.59 & 24322.000 & 3222.1 \\
\hline Sitver & 1.074 & 0.920 & 0.1 & 1.54 & 7.630 & 1.0 & 8.195 & 12.81 & 51.000 & 6.8 & 54.774 & 85.64 & 59.550 & 7.9 \\
\hline Sodium: & 1.000 & 30660.000 & 4061.7 & 73.83 & 7890.000 & 1045.2 & 7890.000 & 19.00 & 2980.000 & 394.8 & 2980.000 & 7.18 & 41530.000 & 5501.7 \\
\hline Strontium & 1.183 & 0.885 & 0.1 & 0.10 & 929.000 & 123.1 & 1099.007 & 99.90 & 0.000 & 0.0 & 0.000 & 0.00 & 929.885 & 123.2 \\
\hline Zine & $1: 245$ & 1.840 & 0.2 & 0.66 & 214.000 & 28.3 & 266.430 & 7711 & 61.700 & 8.2 & 76.817 & 22.23 & 277.540 & 36.8 \\
\hline Zirconium & 1.351 & 0.920 & 0.1 & 1.48 & 7.630 & 1.0 & 10.308 & 12.24 & 53.800 & 7.1 & 72.684 & 86.29 & 62.350 & 8.3 \\
\hline Uranium & 1.202 & 92.000 & 12.2 & 14.42 & 546,000 & 72.3 & 656.292 & 85.58 & 0.000 & 0.0 & 0.000 & 0.00 & 638.000 & 84.5 \\
\hline Nitrate & 1.000 & 50500.000 & 6690.0 & $\leq 100.00$ & NR & NR & & & NR & NR & & & $\geq 50500.000$ & 6690.0 \\
\hline Chloride & 1.000 & 1675.000 & 221.9 & $\leq 100.00$ & NR & NR & & & NR & NR & & & $\geq 1675.000$ & 221.9 \\
\hline Fluoride & 1.000 & 6000.000 & 794.9 & $\leq 100.00$ & NR & NR & & & NR & NR & & & $\geq 6000.000$ & 794.9 \\
\hline Lanthanum & 1.430 & 21.200 & 28 & 0.14 & 15380.000 & 2037.5 & 21993.400 & 99.86 & 0.000 & 0.0 & 0.000 & 0.00 & 15401.200 & 2040.3 \\
\hline Carbonate & 1.000 & 10460.000 & 1385.7 & 65.09 & 5610.000 & 743.2 & 5610.000 & 34.91 & NR & NR & & & 16070.000 & 2128.9 \\
\hline TOC & 2.450 & 1675.800 & 90.6 & 28.93 & 1680.000 & 2226 & 4116.000 & $71: 07$ & NR & NR & & & 2364.000 & 313.2 \\
\hline Nitrite & 1.000 & 881.000 & 116.7 & $\leq 100.00$ & NR & NR & & & NR & NR & & & $\geq 881.000$ & 116.7 \\
\hline Sulfate & 1.000 & 347.000 & 46.0 & 5100.00 & NR & NR & & & NR & NR & & & $=347.000$ & 46.0 \\
\hline Total & & $\begin{array}{r}109813.455 \\
27.73 \\
\end{array}$ & $\begin{array}{l}\text { total solids } \\
\text { wash }\end{array}$ & & & & $\begin{array}{r}264773.482 \\
66.86\end{array}$ & $\begin{array}{l}\text { \%total solids } \\
\text { in acid }\end{array}$ & & & $\begin{array}{r}63550.861 \\
16.05\end{array}$ & $\begin{array}{l}\text { \%total solids } \\
\text { in fusion }\end{array}$ & $110.64 \%$ & $\begin{array}{l}\% \text { mass } \\
\text { balance }\end{array}$ \\
\hline Radionuclides & & $\mathrm{uCi} / \mathrm{g}$ & Ci/tank 9 & 6 dissolved & $\mathrm{uCi} / \mathrm{g}$ & Ci/tank & & \% dissolved & uCi/g & Ci/tank & & \% dissolved & $\mathrm{uCi} / \mathrm{g}$ & Ci/tank \\
\hline Pu-239,240 & & NR & NR & 0000 & NA & 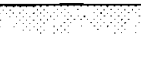 & & 7 & 0.794 & 105.2 & & $\leq 100.00$ & 0.794 & 105.2 \\
\hline $\begin{array}{l}\text { C-14 } \\
\text { Sr.90 }\end{array}$ & & $\begin{array}{r}0.001 \\
\text { NR }\end{array}$ & $\begin{array}{l}0.1 \\
\text { NR }\end{array}$ & 00.00 & NA & & & & $\begin{array}{r}N R \\
2.271\end{array}$ & $\begin{array}{r}N R \\
300.9\end{array}$ & & $<10000$ & $\begin{array}{r}\geq 0.001 \\
2271\end{array}$ & $\begin{array}{r}0.1 \\
3009\end{array}$ \\
\hline Tc-99 & & NR & NR & & NA & & & & 0.002 & 0.3 & & $\leq 100.00$ & 0.002 & 0.3 \\
\hline$A m-241$ & & $<D L$ & & & NA & & & & 0.036 & 4.8 & & $\$ 100.00$ & 0.036 & 4.8 \\
\hline Co-60 & & $<\mathrm{DL}$ & & & NA & & & & 0.030 & 4.0 & & $\leq 100.00$ & 0.030 & 4.0 \\
\hline Cs. 137 & & 0.047 & 62 & $5: 24$ & NA & & & & 0.850 & 112.6 & & 94,76 & 0.897 & 118.8 \\
\hline $\mid-129$ & & NR & NR & & NA & & & & NR & NR & & & NR & NR \\
\hline
\end{tabular}

1. Nitrate on water leach only, would not account for any nitrate associated with cancrinite

2. Total solids for Core 26 were measured as $36.3 \%$ and $37.1 \%$ in one lab and then $41.8 \%$ and $42.0 \%$ in another lab. This suggests that samples samples may have dried before some analyses. This would result in artifically high concentrations.

NR - Not Reported

NA - No Analysis Performed

$<D L$ - Less than Detection Limit 


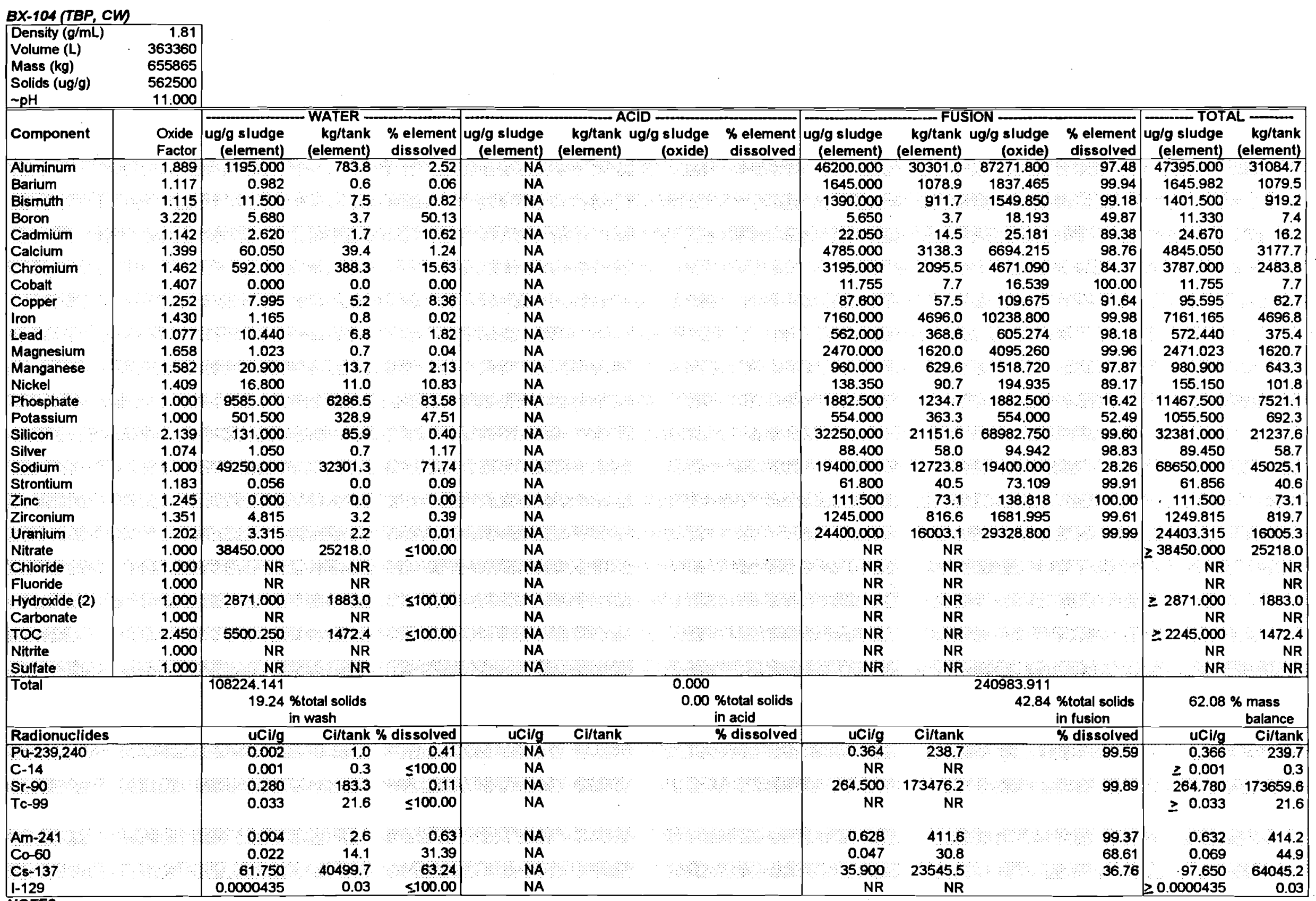

NOTES:

1. Nitrate analysis on water leach only; would not account for nitrate associated with cancrinite

2. OH estimaled based on aluminum concentration in wash.

3. TOC on water leach only

4. Reported value of $4.3 e-5 \mathrm{uCl} / \mathrm{g}$ for I is direct report value for total sample

NR - Not Reported

NA - No Analysis Performed

$<D L$ - Less than Detection Limit 
BX-105 (TBP. CW with $57 t$ of Portland Cement)

\begin{tabular}{|c|c|c|c|c|c|c|c|c|c|c|c|c|c|}
\hline $\begin{array}{l}\text { Density }(\mathrm{g} / \mathrm{mL}) \\
\text { Volume }(\mathrm{L}) \\
\text { Mass }(\mathrm{kg}) \\
\text { Solids (ug/g) } \\
\sim \mathrm{pH} \\
\end{array}$ & $\begin{array}{r}1.69 \\
174110 \\
293375 \\
430000 \\
12.0 \\
\end{array}$ & & & & & & & & & & & & \\
\hline Component & $\begin{array}{l}\text { Oxide } \\
\text { Factor }\end{array}$ & $\begin{array}{r}\text { ug/g s ludge } \\
\text { (element) }\end{array}$ & $\begin{array}{r}- \text { WATER - } \\
\text { kg/tank } \\
\text { (element) }\end{array}$ & $\begin{array}{c}\text { \% element } \\
\text { dissolved }\end{array}$ & $\begin{array}{l}\text { ug/g sludge } \\
\text { (element) }\end{array}$ & $\begin{array}{rr}\mathrm{kg} / \operatorname{tank} \mathrm{ug} / \mathrm{g} \text { sludge } \\
\text { (element) } & \text { (oxide) }\end{array}$ & $\begin{array}{l}\text { \% element } \\
\text { dissolved }\end{array}$ & $\begin{array}{r}\mathbf{u g} / \mathbf{g} \text { sludge } \\
\text { (element) }\end{array}$ & \multicolumn{2}{|c|}{$\begin{array}{l}\text { kg/tank ug/g sludge } \\
\text { (element) }\end{array}$} & $\begin{array}{l}\text { \% element } \\
\text { dissolved }\end{array}$ & $\begin{array}{c}\text { ug/g sludge } \\
\text { (element) }\end{array}$ & $\begin{array}{l}\text { kg/tank } \\
\text { (element) }\end{array}$ \\
\hline Aluminum & 1.889 & 1430.000 & 419.5 & 4.22 & NA & & & 32450.000 & 9520.0 & 61298.050 & $\begin{array}{r}95.78 \\
\end{array}$ & 33880.000 & 9939.6 \\
\hline Barium & 1.117 & 2.040 & 0.6 & 0.06 & NA & & & 3195.000 & 937.3 & 3568.815 & 99.94 & 3197.040 & 937.9 \\
\hline Bismuth & $1: 115$ & 14.200 & 4.2 & 1.83 & NA & & & 762.000 & 223.6 & 849.630 & 98.17 & 776.200 & 227.7 \\
\hline Boron & 3.220 & 5.305 & 1.6 & 100.00 & NA & & & 0.000 & 0.0 & 0.000 & 0.00 & 5.305 & 1.6 \\
\hline Cadmium & 1.142 & 3.220 & 0.9 & 7.27 & NA & & & 41.050 & 12.0 & 46.879 & 92.73 & 44.270 & 13.0 \\
\hline Calcium & 1.399 & 87.600 & 25.7 & 1.41 & NA & & & 6125.000 & 1796.9 & 8568.875 & 98.59 & 6212,600 & 1822.6 \\
\hline Chromium & 1.462 & 151.500 & 44.4 & 175 & NA & & & 8495.000 & 2492.2 & 12419.690 & 98.25 & 8646.500 & 2536.7 \\
\hline Cobalt & 1.407 & 0.570 & 0.2 & 100.00 & NA & & & NR & 0.0 & 0.000 & 0.00 & 0.570 & 0.2 \\
\hline Copper & 1.252 & 2.800 & 0.8 & 10.26 & NA & & & 24.500 & 7.2 & 30.674 & 89.74 & 27.300 & 8.0 \\
\hline Iron & 1.430 & 2.210 & 0.6 & 0.04 & NA & & & 6250.000 & 1833.6 & 8937.500 & 99.96 & 6252.210 & 1834.2 \\
\hline Lead & 1.077 & 42.500 & 12.5 & 7.54 & NA & & & 521.000 & 152.8 & 561.117 & 92.46 & 563.500 & 165.3 \\
\hline Magnesium & 1.658 & 3.980 & 1.2 & 0.12 & NA & & & 3250.000 & 953.5 & 5388.500 & 99.88 & 3253.980 & 954.6 \\
\hline Manganese & 1.582 & 25.750 & 7.6 & 1.72 & NA & & & 1468.000 & 430.7 & 2322.376 & 98.28 & 1493.750 & 438.2 \\
\hline Nickel & 1.409 & 26.150 & 7.7 & 17.80 & NA & & & 120.800 & 35.4 & 170.207 & 82.20 & 146.950 & 43.1 \\
\hline Phosphate & 1.000 & 46170.000 & 13545.1 & 77.27 & NA & & & 13578.000 & 3983.5 & 13578.000 & 22.73 & 59748.000 & 17528.6 \\
\hline Potassium & 1.000 & 785.500 & 230.4 & 54.57 & NA & & & 654.000 & 191.9 & 654.000 & 45.43 & 1439.500 & 422.3 \\
\hline Silicon & 2,139 & 716.000 & 210.1 & 1.73 & NA & & & 40750.000 & 11955.0 & 87164.250 & 98.27 & 41466.000 & 12165.1 \\
\hline Silver & 1.074 & 1.290 & 0.4 & 5.00 & NA & & & 24.500 & 7.2 & 26.313 & 95.00 & 25.790 & 7.6 \\
\hline Sodium & 1.000 & 79300.000 & 23264.7 & 72.06 & NA & & & 30750.000 & $\$ 021.3$ & 30750.000 & 27.94 & 110050.000 & 32286.0 \\
\hline Strontium & 1.183 & 0.714 & 0.2 & 1.27 & NA & & & 55.500 & 16.3 & 65.657 & 98.73 & 56.214 & 16.5 \\
\hline Zinic & 1245 & 0.000 & 0.0 & 0.00 & NA & & & 142.650 & 41.8 & 177599 & 100.00 & 142.650 & 41.8 \\
\hline Zirconium & 1.351 & 5.925 & 1.7 & 1.77 & NA & & & 329.000 & 96.5 & 444.479 & 98.23 & 334.925 & 98.3 \\
\hline Uran & 1202 & 2.590 & 0.8 & 0.06 & NA & & & 4405.000 & 1292.3 & 5294.810 & 99.94 & 4407.590 & 1293.1 \\
\hline Nitrate & 1.000 & 33250.000 & 9754.7 & $\leq 100.00$ & NA & & & NR & NR & & & $\geq 33250.000$ & 9754.7 \\
\hline Chloride & 1.000 & NR & NR & & NA & & & NR & NR & & & NR & NR \\
\hline Fluoride & 1.000 & NR & NR & & NA & & & NR & NR & & & NR & NR \\
\hline Hydroxide & 1.000 & 3600.000 & 1056.2 & $\leq 100.00$ & NA & & & NR & NR & & & $\geq 3600.000$ & 1056.2 \\
\hline Carbonate & 1.000 & NR & NR & & NA & & & NR & NR & & & NR & NR \\
\hline TOC & 2.450 & 6811.000 & 8156 & $\leq 100.00$ & NA & & & NR & NR & & & $\geq 2780.000$ & 815.6 \\
\hline Nitrite & 1.000 & NR & NR & & NA & & & NR & NR & & & NR & NR \\
\hline Sulfate & 1.000 & NR & NR & & $\mathbf{N}$ & & & NR & NR & & & NR & NR \\
\hline \multicolumn{2}{|l|}{ Total } & \multicolumn{3}{|c|}{$\begin{array}{c}172440.844 \\
40.10 \% \text { total solids } \\
\text { in wash }\end{array}$} & \multicolumn{3}{|c|}{$\begin{array}{l}0.000 \\
0.00 \% \text { \%total solids } \\
\text { in acid }\end{array}$} & \multicolumn{4}{|c|}{$\begin{array}{c}242317.421 \\
56.35 \% \text { total solids } \\
\text { in fusion }\end{array}$} & \multicolumn{2}{|c|}{$\begin{array}{c}96.46 \% \text { mass } \\
\text { balance }\end{array}$} \\
\hline \multicolumn{2}{|l|}{ Radionuclides } & uCi/g & Ci/tank & 6 dissolved & uCi/g & $\mathrm{i} / \tan \mathbf{k}$ & \% dissolved & uCi/g & Ci/tank & & $\%$ dissolved & $\mathrm{uCi} / \mathrm{g}$ & Ci/tank \\
\hline & 0.011 & 32 & $\begin{array}{r}3.94 \\
\end{array}$ & NA & & & 0.269 & 78.8 & & 96.06 & 0.280 & 82.0 \\
\hline \multirow{2}{*}{\multicolumn{2}{|c|}{$C-14$}} & 0.001 & 0.3 & $\leq 100.00$ & NA & & & NR & NR & & & 0.001 & 0.3 \\
\hline & & 2.380 & 6982 & -175 & NA & & & 134.000 & 39312,3 & & 98.25 & 136.380 & 40010.5 \\
\hline Tc-99 & & 0.034 & 9.8 & $\leq 100.00$ & NA & & & NR & NR & & & $\geq 0.034$ & 9.8 \\
\hline Am-241 & & 0.012 & 3.5 & 121 & NA & 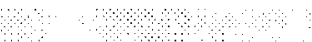 & & 0.982 & 2879 & & & & 291.5 \\
\hline $\mathrm{C}_{0}-60$ & & 0.040 & 11.6 & 25.16 & NA & & & 0.118 & 34.5 & & 74.84 & 0.157 & 46.1 \\
\hline Cs-137 & & 45.100 & 13231.2 & 83.39 & NA & & & 8.980 & 2634.5 & & 16.61 & 54.080 & 158657 \\
\hline$i-129$ & & 0.00003 & 0.01 & $\leq 100.00$ & NA & & & NR & NR & & & $\geq 0.00003$ & 0.01 \\
\hline
\end{tabular}

1. Nitrate on water leach only, would not account for any nitrate associated with cancrinite

2. Hydroxide based on aluminum concentration in wash

3. Reported value of $3.0 \mathrm{e}-5 \mathrm{uCl} / \mathrm{g}$ for I is direct report value for total sample

NR - Not Reported

NA - No Analysis Performed

$<O L$ - Less than Detection Limit 
BX-107 (BIPO4 1C, TBP)

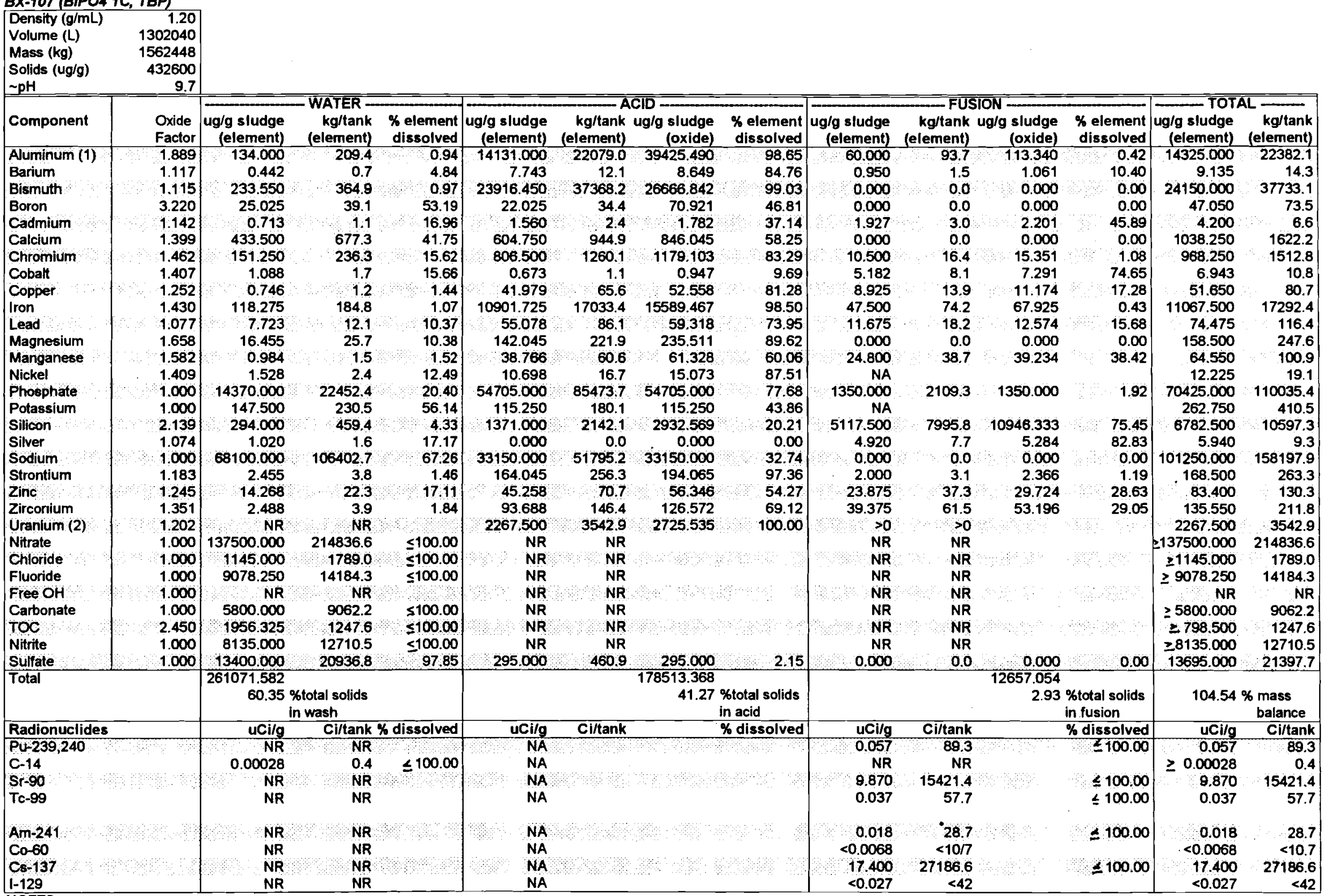

NOTES:

1. Aluminum oxide factor for acid 2.79 (based on $85 \% \mathrm{Al}(\mathrm{OH}) 3$ and $15 \% \mathrm{AIOOH}$ )

2. Uranium analysis on fused sample only; uranium assumed to dissolve with acid

3. With the exception of $\mathrm{C}-14$, all radionuclide analyses were performed on fused samples

NR - Not Reported

4. $\mathrm{Na} 7 \mathrm{~F}(\mathrm{PO} 4) 2$ has been identified in this waste. 


\begin{tabular}{|c|c|c|c|c|c|c|c|c|c|c|c|c|c|c|}
\hline \begin{tabular}{|l|} 
Density $(\mathrm{g} / \mathrm{mL})$ \\
Volume (L) \\
Mass (kg) \\
Solids (ug/g)
\end{tabular} & \begin{tabular}{r|}
1.34 \\
234670 \\
313284 \\
389000 \\
9.8 \\
\end{tabular} & & & \multirow[b]{2}{*}{$\begin{array}{l}\text { \% element } \\
\text { dissolved }\end{array}$} & \multirow[b]{2}{*}{$\begin{array}{r}\text { ug/g sludge } \\
\text { (element) }\end{array}$} & \multirow{2}{*}{\multicolumn{2}{|c|}{ kg/tank ug/g sludge }} & \multirow[b]{2}{*}{$\begin{array}{l}\text { \% element } \\
\text { dissolved }\end{array}$} & \multirow[b]{2}{*}{$\begin{array}{r}\text { ug/g sludge } \\
\text { (element) }\end{array}$} & \multirow{2}{*}{\multicolumn{2}{|c|}{$\begin{array}{l}\text { kg/tank ug/g sludge } \\
\text { (element) }\end{array}$}} & \multirow[b]{2}{*}{$\begin{array}{l}\text { \% element } \\
\text { dissolved }\end{array}$} & \multirow[b]{2}{*}{$\begin{array}{c}\text { ug/g sludge } \\
\text { (element) }\end{array}$} & \multirow[b]{2}{*}{$\begin{array}{r}\overline{\mathrm{kg} / \operatorname{tank}} \\
\text { (element) }\end{array}$} \\
\hline Component & $\begin{array}{l}\text { Oxide } \\
\text { Factor }\end{array}$ & $\begin{array}{r}\text { ug/g sludge } \\
\text { (element) }\end{array}$ & $\begin{array}{r}\text { WATER - } \\
\text { kg/tank } \\
\text { (element) }\end{array}$ & & & & & & & & & & & \\
\hline Aluminum & 1889 & $\begin{array}{r}8.255 \\
\end{array}$ & 2.6 & 0.06 & 6435000 & 2016.0 & 12155.715 & 44.35 & 8065.000 & 2526.6 & 15234.785 & 55.59 & 14508.255 & 4545.2 \\
\hline Barium & 1.117 & 1.835 & 0.6 & 0.04 & 541.500 & 169.6 & 604.856 & 10.91 & 4420.000 & 1384.7 & 4937.140 & 89.05 & 4963.335 & 1554.9 \\
\hline Bismuth & $1: 115$ & 66.850 & 20.9 & 9.26 & 192.000 & 60.2 & 214.080 & 26.60 & 463,000 & 145.1 & 516.245 & 64.14 & 721.850 & 226.1 \\
\hline Boron & 3.220 & NR & NR & & 2.400 & 0.8 & 7.728 & 100.00 & NR & 0.0 & 0.000 & 0.00 & 2.400 & 0.8 \\
\hline Cadmium & 1.142 & 6.900 & 2.2 & 135 & 131.950 & 41.3 & 150.687 & 25.91 & 370.500 & 116.1 & 423.111 & 72.74 & 509.350 & 159.6 \\
\hline Calcium & 1.399 & 37.550 & 11.8 & 0.34 & 789.000 & 247.2 & 1103.811 & 7.10 & 10285.000 & 3222.1 & 14388.715 & 92.56 & 11111.550 & 3481.1 \\
\hline Chromium & 1.462 & 17.600 & 5.5 & 2.85 & 208.500 & 65.3 & 304.827 & 33.76 & 391.500 & 122.7 & 572.373 & 63.39 & 617.600 & 193.5 \\
\hline Cobalt & 1.407 & 36.260 & 11.4 & 56.84 & 4.735 & 1.5 & 6.662 & 7.42 & 22.800 & 7.1 & 32.080 & 35.74 & 63.795 & 20.0 \\
\hline Copper: & 1.252 & 16.300 & 5.1 & 1,91 & 530.500 & 166.2 & 664.186 & 62.06 & 308,000 & 96.5 & 385.616 & 36.03 & 854.800 & 267.8 \\
\hline Iron & 1.430 & 7.020 & 2.2 & 0.01 & 14835.000 & 4647.6 & 21214.050 & 17.12 & 71820.000 & 22500.1 & 102702.600 & 82.87 & 86662.020 & 27149.9 \\
\hline Lead & 1.077 & 25.900 & 8.1 & 0.73 & 1011.500 & 316.9 & 1089.386 & 28.39 & 2525.000 & 791.0 & 2719.425 & 70.88 & 3562.400 & 1116.0 \\
\hline Magnesium & 1.658 & 9.550 & 3.0 & 0.15 & 183.000 & 57.3 & 303.414 & 2.96 & 6000.000 & 1879.7 & 9948.000 & 96.89 & 6192.550 & 1940.0 \\
\hline Manganese & 1.582 & 11.850 & 3.7 & 0.48 & 746.000 & 233.7 & 1180.172 & 30.11 & 1720.000 & 538.8 & 2721.040 & 69.42 & 2477.850 & 776.3 \\
\hline Nickel & 1.409 & 45.100 & 14.1 & 1.58 & 870.500 & 272.7 & 1226.535 & 30.59 & 1930.000 & 604.6 & 2719.370 & 67.82 & 2845.600 & 891.5 \\
\hline Phosphate & 1000 & 2290.500 & 717.6 & 18.28 & 459.000 & 143.8 & 459.000 & 3.66 & 9780.000 & 3063.9 & 9780.000 & 78.06 & 12529.500 & 3925.3 \\
\hline Potassium & 1.000 & 171.000 & 53.6 & 12.18 & 147.800 & 46.3 & 147.800 & 10.53 & 1085.000 & 339.9 & 1085.000 & 77.29 & 1403.800 & 439.8 \\
\hline silicon & 2.139 & 91.700 & 28.7 & 0.13 & 834.500 & 261.4 & 1784.996 & 1.18 & 69692.000 & 21833.4 & 149071.188 & 98.69 & 70618.200 & 22123.6 \\
\hline Silver & 1.074 & 13.300 & 4.2 & 6.00 & 100.400 & 31.5 & 107.830 & 45.32 & 107.850 & 33.8 & 115.831 & 48.68 & 221.550 & 69.4 \\
\hline Sodium & 1.000 & 7311.500 & 2290.6 & 14.48 & 10423.500 & 3265.5 & 10423.500 & 20.64 & 32770.000 & 10266.3 & 32770.000 & 64.88 & 50505.000 & 15822.4 \\
\hline Strontium & 1.183 & 0.000 & 0.0 & 0.00 & 49.700 & 15.6 & 58.795 & 42.39 & 67.550 & 21.2 & 79.912 & 57.61 & 117.250 & 36.7 \\
\hline Zine & 1.245 & 0.000 & 0.0 & 0.00 & 105700 & 33,1 & 131.597 & 60.47 & 69,100 & 21.6 & 86.030 & 39.53 & 174.800 & 54.8 \\
\hline Zirconium & 1.351 & 36.650 & 11.5 & 0.30 & 368.310 & 115.4 & 497.587 & 3.02 & 11805.000 & 3698.3 & 15948.555 & 96.68 & 12209.960 & 3825.2 \\
\hline Uranlum & 1.202 & 1406.500 & 440.6 & 40.83 & 56.500 & 17.7 & 67.913 & 1,64 & 1981,400 & 620.7 & 2381.643 & 57.53 & 3444.400 & 1079.1 \\
\hline Nitrate & 1.000 & 2810.000 & 880.3 & $\leq 100.00$ & NR & NR & & & NR & NR & & & $\geq 2810.000$ & 880.3 \\
\hline Chloride & 1.000 & NR & NR & & NR & NR & & & NR & NR & & & NR & NR \\
\hline Fluoride & 1.000 & NR & NR & & NR & NR & & & NR & NR & & & NR & NR \\
\hline Hydroxide & 1.000 & NR & NR & & NR & NR & & & NR & NR & & & NR & NR \\
\hline Carbonate & 1.000 & NR & NR & & NR & NR & & & NR & NR & & & & NR \\
\hline TOC & 2.450 & 7999.250 & 1022.9 & $\leq 100.00$ & NR & NR & & & NR & NR & & & $\geq 3265.000$ & 1022.9 \\
\hline Nitrite & 1.000 & NR & NR & & NR & NR & & & NR & NR & & & NR & NR \\
\hline Sulfate & 1.000 & NR & $\mathrm{NR}$ & & NR & NR & & & NR & NR & & & NR & NR \\
\hline Total & & $\begin{array}{r}22421.370 \\
5.76 \% \\
\text { if }\end{array}$ & $\begin{array}{l}\text { tal solids } \\
\text { ash }\end{array}$ & & & & $\begin{array}{r}53905.124 \\
13.86\end{array}$ & $\begin{array}{l}\text { \%total solids } \\
\text { in acid }\end{array}$ & & & $\begin{array}{r}368618.657 \\
94.76\end{array}$ & $\begin{array}{l}\text { \%total solids } \\
\text { in fusion }\end{array}$ & 114.38 & $\begin{array}{l}\% \text { mass } \\
\text { balance }\end{array}$ \\
\hline Radionuclides & & uCi/g & Ci/tank & \% dissolved & uCi/g & Ci/tank & & $\%$ dissolved & $\mathrm{uCi} / \mathrm{g}$ & Ci/tank & & \% dissolved & uci/g & Ci/tank \\
\hline $\mathrm{Pu}-239,240$ & & 0.007 & 20 & 0.05 & 8.938 & 2800.0 & & 65.79 & 4.640 & 1453.6 & & 34.16 & 13.584 & 4255.7 \\
\hline C-14 & & NR & NR & & NR & NR & & & NR & NR & & & NR & NR \\
\hline Sr-90 & & 1.039 & 325.5 & 0.04 & 2140.000 & 670428.7 & & 78.97 & 569.000 & 178258.9 & & 21.00 & 2710.039 & 849013.1 \\
\hline TC-99 & & 0.017 & 5.3 & 6.45 & 0.111 & 34.8 & & 42.13 & 0.136 & 42.5 & & 51.42 & 0.264 & 82.6 \\
\hline$A m-241$ & & 0.008 & 25 & 0.59 & 1275 & 399.4 & & 94.41 & 0.068 & 211 & & 5.00 & 1.351 & 423.1 \\
\hline Co-6o & & 0.079 & 24.6 & 1.99 & 0.358 & 112.0 & & 9.06 & 3.510 & 1099.6 & & 88.95 & 3.946 & 1236.2 \\
\hline Cs-137 & & 24.400 & 7644.1 & 41.08 & 17.500 & 5482.5 & & 29.46 & 17.500 & 5482.5 & & 29.46 & 59.400 & 18609.1 \\
\hline $1-129$ & & 0.000011 & 0.01 & $\leq 100.00$ & NR & NR & & & NR & NR & & & $\geq 0.000011$ & 0.01 \\
\hline
\end{tabular}

NOTES:

1. Nitrate on water leach only, would not account for any nitrate associated with cancrinite

2. TOC on water leach only

3. No analyses for nitrite, sulfate, chloride, fluoride, carbonate, total $\mathrm{Cs}$

NR - Not Reported

3. No analyses for nitrite, sulfate, chloride, fluoride, carbonate, total Cs
4. Reported value of $1.1 \mathrm{e}-5 \mathrm{uCV}$ g for $\mathrm{I}$ is direct report value for total sample 


\begin{tabular}{|c|c|c|c|c|c|c|c|c|c|c|c|c|c|c|}
\hline \multirow{2}{*}{$\begin{array}{l}\text { C-104 (CW, POS) } \\
\text { Density (g/mL) } \\
\text { Volume (L) } \\
\text { Mass (kg) } \\
\text { Solids (ug/g) } \\
\text { pH } \\
\end{array}$} & \multirow{2}{*}{\begin{tabular}{r|}
1.21 \\
1116575 \\
1351056 \\
616000 \\
11.500 \\
\\
Oxide \\
Factor
\end{tabular}} & & \multirow[b]{2}{*}{$\begin{array}{l}\text { ug/g sludge } \\
\text { (element) }\end{array}$} & \multirow[b]{2}{*}{$\mathbf{k g} / \tan \mathbf{A}$} & \multirow[b]{2}{*}{$\begin{array}{l}\text { Ug/g sludge } \\
\text { (oxide) }\end{array}$} & \multirow[b]{2}{*}{$\begin{array}{l}\text { \% element } \\
\text { dissolved }\end{array}$} & \multirow[b]{2}{*}{$\begin{array}{l}\text { ug/g sludge } \\
\text { (element) }\end{array}$} & \multirow{2}{*}{\multicolumn{2}{|c|}{$\begin{array}{l}\mathrm{kg} / \text { tank ug/g sludge } \\
\text { (element) }\end{array}$}} & \multirow[b]{2}{*}{$\begin{array}{l}\text { \% element } \\
\text { dissolved }\end{array}$} & \multirow[b]{2}{*}{$\begin{array}{c}\text { ug/g sludge } \\
\text { (element) }\end{array}$} & \multirow[b]{2}{*}{$\begin{array}{l}\mathrm{kg} / \operatorname{tank} \\
\text { (element) }\end{array}$} \\
\hline & & $\begin{array}{l}\text { ug/g sludge } \\
\text { (element) }\end{array}$ & $\begin{array}{r}\text { WATER-- } \\
\text { kg/tank } \\
\text { (element) }\end{array}$ & $\begin{array}{l}\text { \% element } \\
\text { dissolved }\end{array}$ & & & & & & & & & & \\
\hline Aluminum & 1.889 & 541,000 & 730.9 & 1.79 & 8900.000 & 12024.4 & 16812.100 & 29.53 & 20700.000 & 27966.9 & 39102.300 & 68.68 & 30141.000 & 40722.2 \\
\hline Barium & 1.117 & 3.010 & 4.1 & 0.08 & 72.700 & 98.2 & 81.206 & 1.86 & 3830.000 & 5174.5 & 4278.110 & 98.06 & 3905.710 & 5276.8 \\
\hline Bismuth & 1.115 & $60: 100$ & 81.2 & 1.63 & 3470.000 & 4688.2 & 3869.050 & 93.93 & 164.000 & 221.6 & 182.860 & 4.44 & 3694.100 & 4990.9 \\
\hline Boron & 3.220 & 3.210 & 4.3 & 18.76 & 13.900 & 18.8 & 44.758 & 81.24 & 0.000 & 0.0 & 0.000 & 0.00 & 17.110 & 23.1 \\
\hline Cadmium & 1.142 & 65.500 & 88.5 & $5: 11$ & 959.000 & 1295.7 & 1095178 & 74.83 & 257.000 & 347.2 & 293.494 & 20.05 & 1281.500 & 1731.4 \\
\hline Calcium & 1.399 & 21.900 & 29.6 & 0.19 & 1350.000 & 1823.9 & 1888.650 & 11.98 & 9900.000 & 13375.5 & 13850.100 & 87.83 & 11271.900 & 15229.0 \\
\hline Chromium & 1.462 & 45.100 & 60.9 & 4.03 & 746.000 & 1007.9 & 1090.652 & 68.60 & 329.000 & 444.5 & 480.998 & 29.37 & 1120.100 & 1513.3 \\
\hline Cobalt & 1.407 & 0.000 & 0.0 & 0.00 & 9.340 & 12.6 & 13.141 & 57.65 & 6.860 & 9.3 & 9.652 & 42.35 & 16.200 & 21.9 \\
\hline Copper & 1.252 & 3.180 & 4.3 & 2.83 & 74.000 & 100.0 & 92.648 & 66.21 & 34.600 & 46.7 & 43.319 & 30.96 & 111.760 & 151.0 \\
\hline Iron & 1.430 & 6.960 & 9.4 & 0.03 & 19430.000 & 26251.0 & 27784.900 & 74.51 & 6640.000 & 8971.0 & 9495.200 & 25.46 & 26076.960 & 35231.4 \\
\hline Lead & 1.077 & 180.000 & $243: 2$ & 18.05 & 605.000 & 817.4 & 651.585 & 60.68 & 212.000 & 286.4 & 228.324 & 21.26 & 997.000 & 1347.0 \\
\hline Magnesium & 1.658 & 4.610 & 6.2 & 0.08 & 209.000 & 282.4 & 346.522 & 3.83 & 5250.000 & 7093.0 & 8704.500 & 96.09 & 5463.610 & 7381.6 \\
\hline Manganese & 1.582 & 19.900 & 26.9 & 0.60 & 2450.000 & 3310.1 & 3875.900 & 74.29 & 828.000 & 1118.7 & 1309.896 & 25.11 & 3297.900 & 4455.6 \\
\hline Nickel & 1.409 & 77.500 & 104.7 & 4.06 & 1530.000 & 2067.1 & 2155.770 & 80.08 & 303.000 & 409.4 & 426.927 & 15.86 & 1910.500 & 2581.2 \\
\hline Phosphate & 1.000 & 261.000 & 352.6 & 2.78 & 354.000 & 478.3 & 354.000 & 3.78 & 8760.000 & 11835.2 & 8760.000 & 93.44 & 9375.000 & 12666.1 \\
\hline Potassium & 1.000 & 347.000 & 468.8 & 25.76 & 118.000 & 159.4 & 118.000 & 8.76 & 882.000 & 1191.6 & 882.000 & 65.48 & 1347.000 & 1819.9 \\
\hline Silicon & 2.139 & 2140.000 & 28913 & 3.79 & 940.000 & 1270.0 & 2010.660 & 1.67 & 53310.000 & 72024.8 & 114030.090 & 84.54 & 56390.000 & 76186.0 \\
\hline Silver & 1.074 & 1.380 & 1.9 & 0.29 & 293.000 & 395.9 & 314.682 & 62.42 & 175.000 & 236.4 & 187.950 & 37.28 & 469.380 & 634.2 \\
\hline Sodium & 1.000 & 58060.000 & 78442.3 & 60.80 & 9590.000 & 12956.6 & 9590.000 & 10.04 & 27850.000 & 37626.9 & 27850.000 & 29.16 & 95500.000 & 129025.8 \\
\hline Strontium & 1.183 & 0.000 & 0.0 & 0.00 & 29.300 & 39.6 & 34.662 & 36.08 & 51.900 & 70.1 & 61.398 & 63.92 & 81.200 & 109.7 \\
\hline Žine & 1.245 & 0.000 & 0.0 & 0.00 & 72.100 & 97.4 & 89.765 & 61.52 & 45.100 & 60.9 & 56.150 & 38.48 & 117.200 & 158.3 \\
\hline Zirconium & 1.351 & 9.630 & 13.0 & 0.02 & 6768.000 & 9143.9 & 9143.568 & 10.95 & 55020.000 & 74335.1 & 74332.020 & 89.03 & 61797.630 & 83492.0 \\
\hline Uranium & 1.202 & 30.100 & 40.7 & 0.12 & 23260.000 & 31425.6 & 27958.520 & 93.83 & 1500.000 & 2026.6 & 1803.000 & 6.05 & 24790.100 & 33492.8 \\
\hline Nitrate & 1.000 & 23440.000 & 31668.7 & $\leq 100.00$ & NR & NR & & & NR & NR & & & $\geq 23440.000$ & 31668.7 \\
\hline Chloride & 1000 & NR & NR & & $\mathrm{NR}$ & NR & & & NR & NR & & & NR & NR \\
\hline Fluoride & 1.000 & NR & NR & & NR & NR & & & NR & NR & & & NR & NR \\
\hline Hydroxlde & 1.000 & 0.000 & NR & & NR & NR & & & NR & NR & & & NR & \\
\hline Carbonate & 1.000 & NR & NR & & NR & NR & & & NR & NR & & & NR & NR \\
\hline TOC & 2.450 & 10804.500 & 5958.2 & $\$ 100.00$ & NR & NR & & & NR & NR & & & $\geq 4410.000$ & 5958.2 \\
\hline Nitrite & 1.000 & NR & NR & & NR & NR & & & NR & NR & & & NR & NR \\
\hline Sulfate & 1.000 & NR & NR & & NR & NR & & & NR & NR & & & NR & NR \\
\hline Total & & $\begin{array}{r}96125.560 \\
15.60 \\
\end{array}$ & $\begin{array}{l}\text { tal solids } \\
\text { rash }\end{array}$ & & & & $\begin{array}{r}109415.917 \\
17.76\end{array}$ & $\begin{array}{l}\text { \%total solids } \\
\text { in acid }\end{array}$ & & & $\begin{array}{r}306368.287 \\
49.74 \\
\end{array}$ & $\begin{array}{l}\text { \%total solids } \\
\text { in fusion }\end{array}$ & 83.10 & $\begin{array}{l}\% \text { mass } \\
\text { balance }\end{array}$ \\
\hline Radionuclide & & uCi/g & Ci/tank & 6 dissolved & uCi/g & Ci/tank & & \% dissolved & $u \overline{C i} / g$ & Ci/tank & & \% dissolved & $u \overline{C i} / g$ & Ci/tank \\
\hline $\mathrm{Pu}-239,240$ & & 0.004 & 5.4 & 0.04 & 8.680 & 11727.2 & & 89.26 & 1.040 & 1405.1 & & 10.70 & 9.724 & 13137.7 \\
\hline C-14 & & 0.001 & 1.4 & $\leq 100.00$ & NR & NR & & & NR & NR & & & $\geq 0.001$ & 1.4 \\
\hline sr-90 & & 0.029 & 39.2 & 0.01 & 369.000 & 498539.6 & & 99.70 & 1.090 & 1472.7 & & 0.29 & 370,119 & 500051.4 \\
\hline Tc-99 & & 0.023 & 31.1 & 0.83 & 2.370 & 3202.0 & & 85.65 & 0.374 & 505.3 & & 13.52 & 2.767 & 3738.4 \\
\hline$A m-241$ & & 0.006 & 81 & 0.19 & 3110 & 42018 & & 99.68 & 0.004 & 5.4 & & 0.13 & & 4215.3 \\
\hline Co-60 & & 0.027 & 36.5 & 4.84 & 0.412 & 556.6 & & 73.84 & 0.119 & 160.8 & & 21.33 & 0.558 & 753.9 \\
\hline Cs-137 & & 4.964 & 6706.6 & 15.82 & 26.300 & 355328 & & 83.81 & 0.115 & 155.4 & & 0.37 & 31.379 & 42394.8 \\
\hline$[-129$ & & $\geqslant 0.000$ & $\geq 0.0$ & & NR & NR & & & NR & NR & & & $>0.000$ & $\geq 0.0$ \\
\hline
\end{tabular}
NOTES

1. Nitrate on water leach only, would not account for any nitrate associated with cancrinite

2. No analyses for nitrite, sulfate, chloride, fluoride, carbonate, total Cs

3. TOC on water leach only

4. Reported value of $\mathrm{O} \mathrm{uCi} / \mathrm{g}$ for $\mathrm{I}$ is direct report value for total sample

NR - Not Reported

NA - No Analysis Performed

$<D L$ - Less than Detection Limit 


\begin{tabular}{|c|c|c|c|c|c|c|c|c|c|c|c|c|c|c|}
\hline $\begin{array}{l}\text { Density }(\mathrm{g} / \mathrm{mL}) \\
\text { Volume (L) } \\
\text { Mass (kg) } \\
\text { Solids (ug/g) } \\
\sim \mathrm{pH}\end{array}$ & $\begin{array}{r}1.55 \\
567750 \\
880013 \\
623000 \\
\mathrm{NR} \\
\end{array}$ & (drainable liqu & ( pH 9.7) & & & & & & & & & & & \\
\hline \multirow{2}{*}{ Component } & \multirow[b]{2}{*}{$\begin{array}{l}\text { Oxide } \\
\text { Factor }\end{array}$} & \multicolumn{3}{|c|}{ WATER - } & \multirow{2}{*}{$\begin{array}{r}\text { ug/g sludge } \\
\text { (element) }\end{array}$} & \multirow{2}{*}{\multicolumn{2}{|c|}{$\begin{array}{r}\mathrm{kg} / \operatorname{tank} \mathrm{ug} / \mathrm{g} \text { sludge } \\
\text { (element) } \\
\text { (oxide) }\end{array}$}} & \multirow[b]{2}{*}{$\begin{array}{l}\text { \% element } \\
\text { dissolved }\end{array}$} & \multirow{2}{*}{$\begin{array}{r}\text { ug/g sludge } \\
\text { (element) }\end{array}$} & \multirow{2}{*}{\multicolumn{2}{|c|}{$\begin{array}{r}\text { kg/tank ug/g sludge } \\
\text { (element) } \\
\text { (oxide) }\end{array}$}} & \multirow[b]{2}{*}{$\begin{array}{l}\text { \% element } \\
\text { dissolved }\end{array}$} & \multirow{2}{*}{$\begin{array}{c}\text { TOTAI } \\
\text { ug sludge } \\
\text { (element) }\end{array}$} & \multirow{2}{*}{$\begin{array}{c}\mathrm{kg} / \operatorname{tank} \\
\text { (element) }\end{array}$} \\
\hline & & \begin{tabular}{|c} 
ug/g sludge \\
(element)
\end{tabular} & $\begin{array}{r}\mathbf{k g} / \operatorname{tank} \\
\text { (element) }\end{array}$ & $\begin{array}{c}\% \text { element } \\
\text { dis solved }\end{array}$ & & & & & & & & & & \\
\hline Aluminum & 1.889 & 102.000 & 89.8 & 0.16 & 37910.000 & 33361.3 & 71611.990 & 61.30 & 23830.000 & 20970.7 & 45014.870 & 38.53 & 61842.000 & 54421.7 \\
\hline Barium & 1.117 & 3.530 & 3.1 & 0.14 & 70.300 & 61.9 & 78.525 & 2.74 & 2490.000 & 2191.2 & 2781.330 & 97.12 & 2563.830 & 2256.2 \\
\hline Bismuth & 1.115 & 70.500 & 62.0 & 11.28 & 480.000 & 422.4 & 535.200 & 76.80 & 74.500 & 65.6 & 83.068 & 11.92 & 625.000 & 550.0 \\
\hline Boron & 3.220 & 3.230 & 2.8 & 26.24 & 9.080 & 8.0 & 29.238 & 73.76 & 0.000 & 0.0 & 0.000 & 0.00 & 12.310 & 10.8 \\
\hline Cadmium & 1.142 & 56.400 & 49.6 & 35.63 & 42.300 & 37.2 & 48.307 & 26.72 & 59.600 & 52.4 & 68.063 & 37.65 & 158.300 & 139.3 \\
\hline Calcium & 1.399 & 71.700 & 63.1 & 0.92 & 798.000 & 702.2 & 1116.402 & 10.23 & 6930.000 & 6098.5 & 9695.070 & 88.85 & 7799.700 & 6863.8 \\
\hline Chromium & 1.462 & 468.000 & 411.8 & 49.62 & 410.000 & 360.8 & 599.420 & 43.47 & 65.200 & 57.4 & 95.322 & 6.91 & 943.200 & 830.0 \\
\hline Cobalt & 1.407 & 0.000 & 0.0 & 0.00 & 10.300 & 9.1 & 14.492 & 100.00 & 0.000 & 0.0 & 0.000 & 0.00 & 10.300 & 9.1 \\
\hline Copper & 1.252 & 0.000 & 0.0 & 0.00 & 64.200 & 56.5 & 80.378 & 41.50 & 90.500 & 79.6 & 113.306 & 58.50 & 154.700 & 136.1 \\
\hline Iron & 1.430 & 5.170 & 4.5 & 0.05 & 6390.000 & 5623.3 & 9137.700 & 60.25 & 4210.000 & 3704.9 & 6020.300 & 39.70 & 10605.170 & 9332.7 \\
\hline Lead & 1.077 & 212.000 & 186.6 & 23.61 & 463.000 & 407.4 & 498.651 & 51.56 & 223.000 & 196.2 & 240.171 & 24.83 & 898.000 & 790.3 \\
\hline Magnesium & 1.658 & 24.400 & 21.5 & 0.66 & 219.000 & 192.7 & 363.102 & 5.93 & 3450.000 & 3036.0 & 5720.100 & 93.41 & 3693.400 & 3250.2 \\
\hline Manganese & 1.582 & 23.300 & 20.5 & 0.94 & 2240.000 & 1971.2 & 3543.680 & 90.60 & 209.000 & 183.9 & 330.638 & 8.45 & 2472.300 & 2175.7 \\
\hline Nickel & 1.409 & 6.000 & 5.3 & 0.28 & 1900.000 & 1672.0 & 2677.100 & 88.74 & 235.000 & 206.8 & 331.115 & 10.98 & 2141.000 & 1884.1 \\
\hline Phosphate & 1.000 & 3960.000 & 3484.8 & 52.11 & 1941000 & 1708.1 & 1941.000 & 25.54 & 1698.000 & 1494.3 & 1698.000 & 22.35 & 7599.000 & 6687.2 \\
\hline Potassium & 1.000 & 385.000 & 338.8 & 34.78 & 145.000 & 127.6 & 145.000 & 13.10 & 577.000 & 507.8 & 577.000 & 52.12 & 1107.000 & 974.2 \\
\hline Sillicon & 2.139 & 49.000 & 43.1 & 0.12 & 343.000 & 301.8 & 733.677 & 0.86 & 39470.000 & $34734: 1$ & 84426.330 & 99.02 & 39862.000 & 35079.1 \\
\hline Silver & 1.074 & 1.720 & 1.5 & 2.57 & 58.400 & 51.4 & 62.722 & 87.20 & 6.850 & 6.0 & 7.357 & 10.23 & 66.970 & 58.9 \\
\hline Sodium & 1.000 & 34910.000 & 307212 & 48.64 & 16570.000 & 14581.8 & 16570.000 & 23.09 & 20290.000 & 17855.5 & 20290.000 & 28.27 & 71770.000 & 63158.5 \\
\hline Strontium & 1.183 & 0.000 & 0.0 & 0.00 & 160.000 & 140.8 & 189.280 & 62.84 & 94.600 & 83.2 & 111.912 & 37.16 & 254.600 & 224.1 \\
\hline Zinc & 1,245 & 0.000 & 0.0 & 0.00 & 59.100 & 52.0 & 73.580 & 7.94 & 685.000 & 602.8 & 852.825 & 92.06 & 744.100 & 654.8 \\
\hline Zirconium & 1.351 & 11.300 & 9.9 & 1.53 & 18.200 & 16.0 & 24.588 & 2.47 & 707.000 & 622.2 & 955.157 & 95.99 & 736.500 & 648.1 \\
\hline Uranium & 1.202 & 642.000 & 565.0 & 5.37 & 11310,000 & 9952.9 & 13594.620 & 94.63 & NR & NR & & & 11952.000 & 10517.9 \\
\hline Nitrate & 1.000 & 10490.000 & 9231.3 & $\leq 100.00$ & NR & NR & & & NR & NR & & & $=10490.000$ & 9231.3 \\
\hline Chloride & 1.000 & NR & NR & & NR & NR & & & NR & NR & & & NR & NR \\
\hline Fluoride & 1.000 & NR & NR & & NR & NR & & & NR & NR & & & NR & NR \\
\hline Hydroxide & 1.000 & NR & NR & & NR & NR & & & NR & NR & & & NR & NR \\
\hline Carbonate & 1.000 & NR & NR & & NR & NR & & & NR & NR & & & NR & NR \\
\hline TOC & 2,450 & 2082.500 & 748.0 & 5100.00 & NR & NR & & & NR & NR & & & $\geq 850.000$ & 748.0 \\
\hline Nitrite & 1.000 & NR & NR & & NR & NR & & & NR & NR & & & NR & NR \\
\hline Sulfate & 1.000 & NR & NR & & NR & NR & & & NR & NR & & & NR & NR \\
\hline \multicolumn{2}{|l|}{ Total } & \multicolumn{3}{|c|}{$\begin{array}{l}53577.750 \\
8.60 \% \text { total solids } \\
\text { in wash }\end{array}$} & \multicolumn{4}{|c|}{$\begin{array}{c}123668.651 \\
19.85 \% \text { total solids } \\
\text { in acid }\end{array}$} & \multicolumn{4}{|c|}{$\begin{array}{c}179411.934 \\
28.80 \% \text { total solids } \\
\text { in fusion }\end{array}$} & \multicolumn{2}{|c|}{$\begin{array}{c}57.25 \% \text { mass } \\
\text { balance }\end{array}$} \\
\hline \multicolumn{2}{|l|}{ Radionuclides } & uCi/g & Ci/tank & $\%$ dissolved & $\mathrm{uCi} / \mathrm{g}$ & Ci/tank & & $\%$ dissolved & $\mathrm{uCi} / \mathrm{g}$ & Ci/tank & & \% dissolved & uCi/g & Ci/tank \\
\hline \multirow{2}{*}{\multicolumn{2}{|c|}{$\begin{array}{l}\text { Pu-239,240 } \\
\text { C-14 }\end{array}$}} & 0.004 & 3.5 & 0.13 & 2.900 & 2552.0 & & 94.74 & 0.157 & 138.2 & & 5.13 & 3.061 & 2693.7 \\
\hline & & 0.001 & 0.9 & $\leq 100.00$ & NR & NR & & & NR & NR & & & $\geq 0.001$ & 0.9 \\
\hline Sr-90: & & 0.430 & 378.4 & 0.05 & 863.000 & 759450.8 & & 99.93 & 0.172 & 151.4 & & 0.02 & $863: 602$ & 759980.6 \\
\hline Tc-99 & & 0.068 & 59.8 & 64.76 & 0.032 & 28.2 & & 30.48 & 0.005 & 4.4 & & 4.76 & 0.105 & 92.4 \\
\hline$A m-241$ & & 1128 & 9927 & 7475 & 0376 & 330.9 & & 24.92 & 0.005 & 4.4 & & 0.33 & 1.509 & 1327.9 \\
\hline Co-60 & & 0.027 & 23.8 & 3.55 & 0.636 & 559.7 & & 83.57 & 0.098 & 86.2 & & 12.88 & 0.761 & 669.7 \\
\hline Cs-137 & & 12,000 & 10560.2 & 0.15 & 135.000 & 118801.7 & & 9171 & 0.206 & 181.3 & & 0.14 & 147.206 & 129543.1 \\
\hline $\mid-129$ & & 0.000 & 0.1 & $\leq 100.00$ & NR & NR & & & NR & NR & & & $\geq 0.000$ & 0.1 \\
\hline
\end{tabular}

NOTES:

1. Nitrate on water leach only, would not account for any nitrate associated with cancrinite

2. TOC on water leach only

3. No analyses for nitrite, sulfate, chloride, fluoride, carbonate, total $\mathrm{Cs}$

NR - Not Reported

4. Reported value of $1.2 \mathrm{e}-4$ uCivg for I is direct report value for total sample 


\begin{tabular}{|c|c|c|c|c|c|c|c|c|c|c|c|c|c|c|}
\hline \begin{tabular}{|l} 
Density (g/mL) \\
Volume (L) \\
Mass (kg) \\
Solids (ug/g) \\
pH \\
\end{tabular} & $\begin{array}{r}1.43 \\
745645 \\
1066272 \\
475000 \\
10.5 \\
\end{array}$ & & & & & & & & & & & & & \\
\hline Component & $\begin{array}{l}\text { Oxide } \\
\text { Factor }\end{array}$ & $\begin{array}{r}\text { ug/g s ludge } \\
\text { (element) }\end{array}$ & $\begin{array}{r}- \text { WATER - } \\
\text { kg/tank } \\
\text { (element) }\end{array}$ & $\begin{array}{l}\text { \% element } \\
\text { dissolved }\end{array}$ & $\begin{array}{r}\text { ug/g sludge } \\
\text { (element) }\end{array}$ & $\begin{array}{r}\text { kg/tank } \\
\text { (element) }\end{array}$ & $\begin{array}{l}\text { ug/g sludge } \\
\text { (oxide) }\end{array}$ & $\begin{array}{l}\text { \% element } \\
\text { dissolved }\end{array}$ & $\begin{array}{l}\text { ug/g s ludge } \\
\text { (element) }\end{array}$ & $\begin{array}{r}\text { kg/tank } \\
\text { (element) }\end{array}$ & $\begin{array}{r}\text { ug/g sludge } \\
\text { (oxide) }\end{array}$ & $\begin{array}{l}\text { \% element } \\
\text { dissolved }\end{array}$ & $\begin{array}{r}\text { TOT/g sludge } \\
\text { (element) }\end{array}$ & $\begin{array}{l}\text { AL } \overline{\text { kg/tank }} \\
\text { (element) }\end{array}$ \\
\hline Aluminum & 1.889 & 21.500 & 22.9 & 0.05 & 32870.000 & 35048.4 & 62091.430 & 80.32 & 8030.000 & 8562.2 & 15168.670 & 19.62 & 40921.500 & 43633.5 \\
\hline Barium & 1.117 & 2.890 & 3.1 & 0.06 & 123.000 & 131.2 & 137.391 & 2.52 & 4760.000 & 5075.5 & 5316.920 & 97.42 & 4885.890 & 5209.7 \\
\hline Bismuth & 1.115 & 15.000 & 160 & 3.00 & 52.700 & 56.2 & 58.761 & 10.53 & 433.000 & 461.7 & 482.795 & 86.48 & 500.700 & 533.9 \\
\hline Boron & 3.220 & 4.770 & 5.1 & 24.50 & 14.700 & 15.7 & 47.334 & 75.50 & 0.000 & 0.0 & 0.000 & 0.00 & 19.470 & 20.8 \\
\hline Cadmium & 1.142 & 12.000 & 12.8 & 3.24 & 12.000 & 12.8 & 13.704 & 3.24 & 346.000 & 368.9 & 395.132 & 93.51 & 370.000 & 394.5 \\
\hline Calcium & 1.399 & 45.600 & 48.6 & 0.38 & 739.000 & 788.0 & 1033.861 & 6.20 & 11140.000 & 11878.3 & 15584.860 & 93.42 & 11924.600 & 12714.9 \\
\hline Chromium & 1.462 & 1.420 & 1.5 & 0.14 & 611.000 & 651.5 & 893.282 & 62.07 & 372,000 & 396.7 & 543.864 & 37.79 & 984.420 & 1049.7 \\
\hline Cobalt & 1.407 & 0.000 & 0.0 & 0.00 & 4.810 & 5.1 & 6.768 & 100.00 & 0.000 & 0.0 & 0.000 & 0.00 & 4.810 & 5.1 \\
\hline Copper & 1.252 & 1.520 & 1.6 & 119 & 67.500 & 72.0 & 84.510 & 52.73 & 59.000 & 62.9 & 73.868 & 46.09 & 128.020 & 136.5 \\
\hline Iron & 1.430 & 1.110 & 1.2 & 0.00 & 27460.000 & 29279.8 & 39267.800 & 52.75 & 24600.000 & 26230.3 & 35178.000 & 47.25 & 52061.110 & 55511.3 \\
\hline Lead & 1.077 & 44.900 & 47.9 & 1.87 & 1060.000 & 1130.2 & 1141.620 & 44.08 & 1300.000 & 1386.2 & $1400: 100$ & 54.06 & 2404.900 & 2564.3 \\
\hline Magnesium & 1.658 & 11.400 & 12.2 & 0.17 & 138.000 & 147.1 & 228.804 & 2.10 & 6410.000 & 6834.8 & 10627.780 & 97.72 & 6559.400 & 6994.1 \\
\hline Manganese & 1.582 & 4.940 & 5.3 & 0.27 & 1100.000 & 1172.9 & 1740.200 & 59.75 & 736.000 & 784.8 & 1164.352 & 39.98 & 1840.940 & 1962.9 \\
\hline Nickel & 1.409 & 32.200 & 34.3 & 3.31 & 488.000 & 520.3 & 687.592 & 50.14 & 453.000 & 483.0 & 638.277 & 46.55 & 973.200 & 1037.7 \\
\hline Phosphate & 1.000 & 1320.000 & 1407.5 & 15.12 & 3240.000 & 3454.7 & 3240.000 & 37.11 & 4170.000 & 4446.4 & 4170.000 & 47.77 & 8730.000 & 9308.6 \\
\hline Potassium & 1.000 & 156.000 & 166.3 & 10.58 & 89.000 & 94.9 & 89.000 & 6.03 & 1230.000 & 1311.5 & 1230.000 & 83.39 & 1475.000 & 1572.8 \\
\hline Silicon & 2.139 & 24.700 & 26.3 & 0.03 & 147.000 & 156.7 & 314.433 & 0.21 & 70840.000 & 75534.7 & 151526.760 & 99.76 & 71011.700 & 75717.8 \\
\hline Silver & 1.074 & 1.360 & 1.5 & 0.26 & 349.000 & 372.1 & 374.826 & 66.05 & 178.000 & 189.8 & 191.172 & 33.69 & 528.360 & 563.4 \\
\hline Sodium & 1.000 & 45480.000 & 48494.1 & 38.84 & 36650.000 & 39078.9 & 36650.000 & 31.30 & 34980.000 & 37298.2 & 34980.000 & 29.87 & 117110.000 & 124871.2 \\
\hline Strontium & 1.183 & 0.000 & 0.0 & 0.00 & 42.400 & 45.2 & 50.159 & 41.17 & 60.600 & 64.6 & 71.690 & 58.83 & 103.000 & 109.8 \\
\hline Zine & 1.245 & 0.000 & 0.0 & 0.00 & 46.300 & 49.4 & 57.644 & 100.00 & 0.000 & 0.0 & 0.000 & 0.00 & 46.300 & 49.4 \\
\hline Zirconium & 1.351 & 113.000 & 120.5 & 5.22 & 3.700 & 3.9 & 4.999 & 0.17 & 2050.000 & 2185.9 & 2769.550 & 94.61 & 2166.700 & 2310.3 \\
\hline Uranium & 1.202 & 9.130 & 9.7 & 2.23 & 2.770 & 3.0 & 3.330 & 0.68 & 397.000 & 423.3 & $477: 194$ & 97.09 & 408.900 & 436.0 \\
\hline Nitrate & 1.000 & 928.000 & 989.5 & $\leq 100.00$ & NR & NR & & & NR & NR & & & $\geq 928.000$ & 989.5 \\
\hline Chiloride & 1.000 & NR & NR & & NR & NR & & & NR & NR & & & NR & NR \\
\hline Fluoride & 1.000 & NR & NR & & NR & NR & & & NR & NR & & & NR & NR \\
\hline Hydroxide & 1.000 & NR & NR & & NR & NR & & & NR & NR & & & NR & \\
\hline Carbonate & 1.000 & NR & NR & & NR & NR & & & NR & NR & & & NR & \\
\hline TOC & 2.450 & 11319.000 & 49262 & $\leq 100.00$ & NR & NR & & & NR & NR & & & $\geq 4620.000$ & 4926.2 \\
\hline Nitrite & 1.000 & NR & NR & & NR & NR & & & NR & NR & & & NR & NR \\
\hline Sulfate & 1.000 & NR & NR & & NR & NR & & & NR & NR & & & NR & NR \\
\hline Total & & $\begin{array}{r}59550.440 \\
12.54\end{array}$ & $\begin{array}{l}\text { btotal solids } \\
\text { wash }\end{array}$ & & & & $\begin{array}{r}148217.446 \\
31.20 \\
\end{array}$ & $\begin{array}{l}\text { \%total solids } \\
\text { in acid }\end{array}$ & & & $\begin{array}{r}281990.984 \\
59.37 \\
\end{array}$ & $\begin{array}{l}\text { \%total solids } \\
\text { in fusion }\end{array}$ & 103.11 & $\begin{array}{l}\% \text { mass } \\
\text { balance }\end{array}$ \\
\hline Radionuclides & & $\mathrm{uCi} / \mathrm{g}$ & Ci/tank & $\%$ dissolved & uCi/g & Ci/tank & & \% dissolved & uCi/g & Ci/tank & & \% dissolved & uCi/g & Ci/tank \\
\hline $\begin{array}{l}\text { Pu-239,240 } \\
\text { C-14 }\end{array}$ & & $\begin{array}{r}0.245 \\
\text { NR }\end{array}$ & $\begin{array}{c}261 / 2 \\
\text { NR }\end{array}$ & 4.77 & $\begin{array}{r}2,440 \\
\text { NR }\end{array}$ & $\begin{array}{c}26017 \\
\text { NR }\end{array}$ & & 47.52 & $\begin{array}{r}2.450 \\
\text { NR }\end{array}$ & $\begin{array}{r}2612.4 \\
\text { NR }\end{array}$ & & 47.71 & $\begin{array}{r}5.135 \\
\text { NR }\end{array}$ & $\begin{array}{r}5475.3 \\
\text { NR }\end{array}$ \\
\hline Sr 90 & & 0.204 & 2175 & 0.01 & 1970,000 & 2100556.5 & & 9914 & 16.800 & 17913.4 & & 0.85 & 1987.004 & 2118687.4 \\
\hline Tc-99 & & 0.035 & 37.3 & 16.06 & 0.103 & 109.8 & & 47.25 & 0.080 & 85.3 & & 36.70 & 0.218 & 232.4 \\
\hline Am-241 & & 0.015 & 16.0 & 142 & 0.844 & 899.9 & & 80.15 & 0.194 & 206.9 & & 18.42 & 1.053 & 1122.8 \\
\hline Co-60 & & 0.028 & 29.9 & 3.15 & 0.333 & 355.1 & & 37.50 & 0.527 & 561.9 & & 59.35 & 0.888 & 946.8 \\
\hline Cs-137 & & 62200 & 663221 & 18,83 & 239.000 & 2548391 & & 72,36 & 29.100 & 31028.5 & & 8.81 & 330.300 & 352189.8 \\
\hline$i-129$ & & 0.000081 & 0.1 & $\leq 100.00$ & NR & NR & & & NR & NR & & & $\geq 0.000081$ & 0.1 \\
\hline
\end{tabular}

NOTES:

1. Nitrate on water leach only, would not account for any nitrate associated with cancrinite

2. TOC on water leach only

3. No analyses for nitrite, sulfate, chloride, fluoride, carbonate, total $\mathrm{Cs}_{8}$

4. Reported value of $8.1 \mathrm{e}-5 \mathrm{uCi} / \mathrm{g}$ for $\mathrm{I}$ is direct report value for total sample

NR - Not Reported

NA - No Analysis Performed

$<D L$ - Less than Detection Limit 


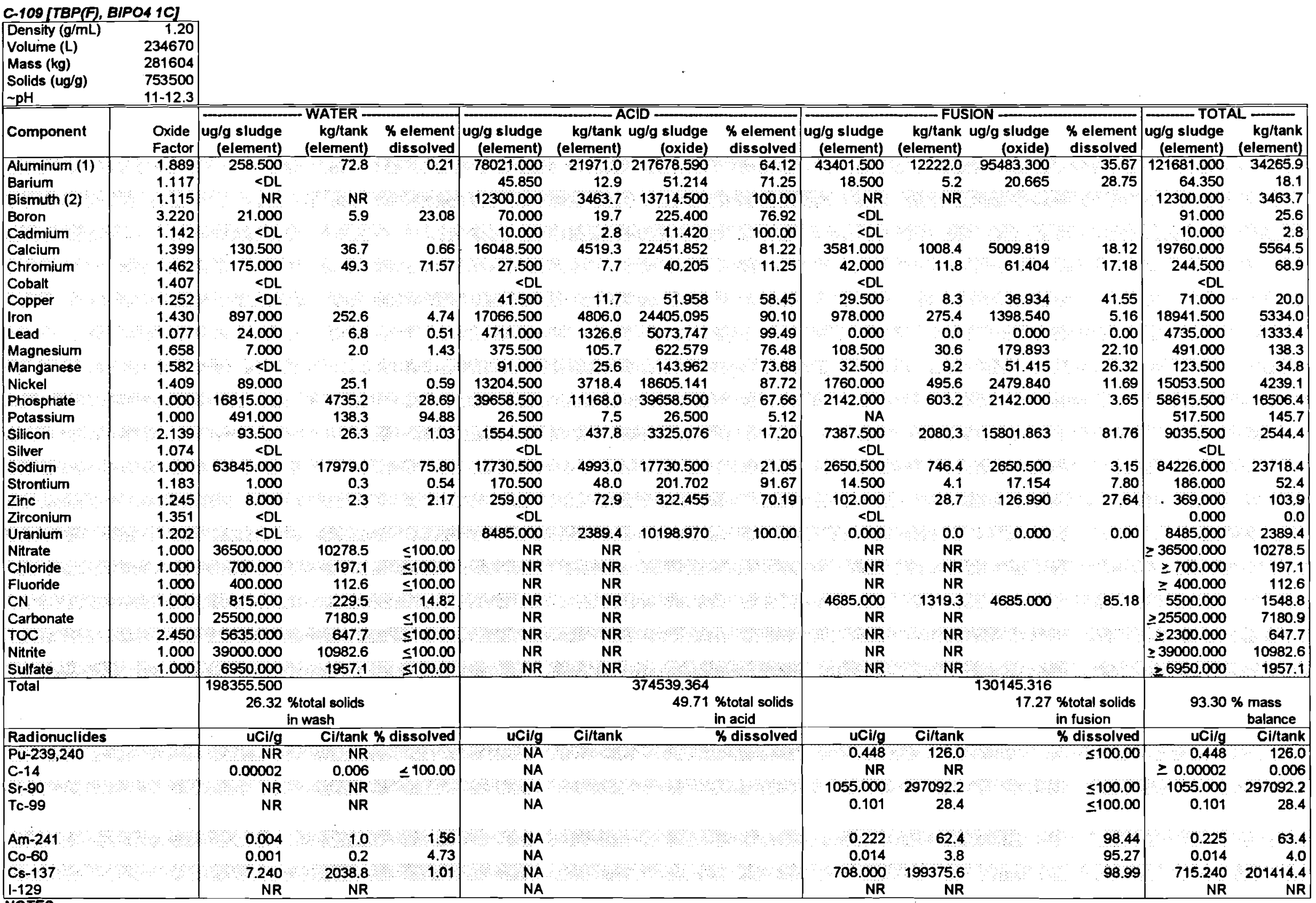

NOTES:

1. Acid soluble oxide factor $2.79(85 \% \mathrm{Al}(\mathrm{OH}) 3,15 \% \mathrm{AlOOH})$; fusion soluble oxide factor $2.22(\mathrm{AlOOH})$

2. Bismuth concentration estimated based on work by Lumetta, et al PNL-9387

3. Nitrate and sulfate on water leach only, would not account for nitrate in cancrinite (I.D.'d by XRD) or water insoluble sulfate

NR - Not Reported

NA - No Analysis Performed

$<D L$ - Less than Detection Limit 


\begin{tabular}{|c|c|c|c|c|c|c|c|c|c|c|c|c|c|c|}
\hline \begin{tabular}{|l|} 
Density $(\mathrm{g} / \mathrm{mL})$ \\
Volume (L) \\
Mass (kg) \\
Solids (ug/g) \\
$\sim \mathrm{pH}$
\end{tabular} & $\begin{array}{r}1.20 \\
707795 \\
849354 \\
401600 \\
11.0 \\
\end{array}$ & & & & & & & & & & & & & \\
\hline \multirow[b]{2}{*}{ Component } & \multirow[b]{2}{*}{$\begin{array}{l}\text { Oxide } \\
\text { Factor }\end{array}$} & \multicolumn{3}{|c|}{ - WATER - } & \multirow[b]{2}{*}{$\begin{array}{l}\text { ug/g sludge } \\
\text { (element) }\end{array}$} & \multirow{2}{*}{$\begin{array}{r}\text { kg/tank } \\
\text { (element) }\end{array}$} & \multirow{2}{*}{$\begin{array}{r}\text { ug/g sludge } \\
\text { (oxide) }\end{array}$} & \multirow[b]{2}{*}{$\begin{array}{l}\text { \% element } \\
\text { dissolved }\end{array}$} & \multirow{2}{*}{$\begin{array}{l}\text { ug/g sludge } \\
\text { (element) }\end{array}$} & \multirow{2}{*}{\multicolumn{2}{|c|}{$\begin{array}{r}\mathrm{kg} / \operatorname{tank} \text { ug/g sludge } \\
\text { (element) } \\
\text { (oxide) }\end{array}$}} & \multirow[b]{2}{*}{$\begin{array}{l}\text { \% element } \\
\text { dissolved }\end{array}$} & \multirow{2}{*}{$\begin{array}{c}\text { ug/g sludge } \\
\text { (element) }\end{array}$} & \multirow{2}{*}{$\begin{array}{r}\text { kg/tank } \\
\text { (element) }\end{array}$} \\
\hline & & $\begin{array}{r}\text { ug/g sludge } \\
\text { (element) }\end{array}$ & $\begin{array}{r}\text { kg/tank } \\
\text { (element) }\end{array}$ & $\begin{array}{l}\text { \% element } \\
\text { dissolved }\end{array}$ & & & & & & & & & & \\
\hline \begin{tabular}{|l|} 
Aluminum (1) \\
\end{tabular} & 1.889 & 1210.000 & 1027.7 & 8.31 & 13350.000 & 11338.9 & 37246.500 & 91.69 & 0.000 & 0.0 & 0.000 & 0.00 & 14560.000 & 12366.6 \\
\hline Barium & 1.117 & 0.880 & 0.7 & 11.67 & 6.658 & 5.7 & 7.437 & 88.33 & 0.000 & 0.0 & 0.000 & 0.00 & 7.538 & 6.4 \\
\hline Bismuth & 1.115 & 117.800 & 100.1 & 0.71 & 16382200 & 13914.3 & 18266.153 & 99.29 & 0.000 & 0.0 & 0.000 & 0.00 & 16500.000 & 14014.3 \\
\hline Boron & 3.220 & 24.860 & 21.1 & 100.00 & 0.000 & 0.0 & 0.000 & 0.00 & 0.000 & 0.0 & 0.000 & 0.00 & 24.860 & 21.1 \\
\hline Cadmium & 1.142 & 0.812 & 0.7 & 15.62 & 2.116 & 1.8 & 2.416 & 40.71 & 2.270 & 1.9 & 2.592 & 43.67 & 5.198 & 4.4 \\
\hline Calcium & 1.399 & 187.800 & 159.5 & 29.54 & 269.800 & 229.2 & 377.450 & 42.43 & 178.200 & 151.4 & 249.302 & 28.03 & 635.800 & 540.0 \\
\hline Chromium & 1.462 & 259.600 & 220.5 & 55.64 & 207.000 & 175.8 & 302.634 & 44.36 & 0.000 & 0.0 & 0.000 & 0.00 & 466.600 & 396.3 \\
\hline Cobalt & 1.407 & 2.725 & 2.3 & 44.58 & 0.000 & 0.0 & 0.000 & 0.00 & 3.387 & 2.9 & 4.766 & 55.42 & 6.112 & 5.2 \\
\hline Copper & 1.252 & 2.338 & 2.0 & 2.84 & 28.382 & 24.1 & 35.535 & 34.54 & 51.460 & 43.7 & 64.428 & 62.62 & 82.180 & 69.8 \\
\hline Iron & 1.430 & 310.200 & 263.5 & 2.83 & 10541.800 & 9038.7 & 15217.774 & 97.17 & 0.000 & 0.0 & 0.000 & 0.00 & 10952.000 & 9302.1 \\
\hline Lead & 1.077 & 19.828 & 16.8 & 7.42 & 225.572 & 191.6 & 242.941 & 84.42 & 21.800 & 18.5 & 23.479 & 8.16 & 267.200 & 226.9 \\
\hline Magnesium & 1.658 & 14.166 & 12.0 & 9.51 & 134.834 & 114.5 & 223.555 & 90.49 & 0.000 & 0.0 & 0.000 & 0.00 & 149.000 & 126.6 \\
\hline Manganese & 1.582 & 2.078 & 1.8 & 3.92 & 33.442 & 28.4 & 52.905 & 63.05 & 17.522 & 14.9 & 27.720 & 33.03 & 53.042 & 45.1 \\
\hline Nickel & 1.409 & 2.777 & 2.4 & 11.48 & 21.403 & 18.2 & 30.157 & 88.52 & NA & & & & 24.180 & 20.5 \\
\hline Phosphate & 1.000 & 24041.400 & 20419.7 & 38.86 & 32958.600 & 27993.5 & 32958.600 & 53.28 & 4860.000 & 4127.9 & 4860.000 & 7.86 & 61860.000 & 52541.0 \\
\hline Potassium & 1.000 & 396.726 & 337.0 & 69.92 & 170.674 & 145.0 & 170.674 & 30.08 & NA & & & & 567.400 & 481.9 \\
\hline Sillicon & 2.139 & 240.280 & 204.1 & 3.41 & 432.720 & 367.5 & 925.588 & 6.13 & 6381.000 & 5419.7 & 13648.859 & 90.46 & 7054.000 & 5991.3 \\
\hline Silver & 1.074 & 1.148 & 1.0 & 23.01 & 0.000 & 0.0 & 0.000 & 0.00 & 3.842 & & 4.126 & 76.99 & 4.990 & 4.2 \\
\hline Sodium & 1.000 & 66168.000 & 56200.1 & 79.05 & 10272.000 & 8724.6 & 10272.000 & 12.27 & 7260.000 & 6166.3 & 7260.000 & 8.67 & 83700.000 & 71090.9 \\
\hline Strontium & 1.183 & 5.534 & 4.7 & 4.40 & 118.786 & 100.9 & 140.524 & 94.41 & 1.500 & 1.3 & 1.775 & 1.19 & 125.820 & 106.9 \\
\hline Zinc & 1.245 & 11.256 & 9.6 & 5.14 & 136.464 & 115.9 & 169.898 & 62.37 & 71.080 & 60.4 & 88.495 & 32.49 & 218.800 & 185.8 \\
\hline Zirconium & 1.351 & 12.442 & 10.6 & 7.45 & 26.598 & 22.6 & 35.934 & 15.93 & 127.960 & 108.7 & 172.874 & 76.62 & 000 & 141.8 \\
\hline Uranium (2) & 1.202 & NR & NR & & 1508,000 & 1280.8 & 1812.616 & 100.00 & $>0$ & $>0$ & & & 1508.000 & 1280.8 \\
\hline Nitrate & 1.000 & 109080.000 & 92647.5 & $\leq 100.00$ & NR & NR & & & NR & NR & & & $=109080.000$ & 92647.5 \\
\hline Chloride & 1.000 & 1377000 & 1169.6 & $\leq 100.00$ & NR & NR & & & NR & NR & & & $\geq 1377.000$ & 1169.6 \\
\hline Fluoride & 1.000 & 7520.000 & 6387.1 & $\leq 100.00$ & NR & NR & & & NR & NR & & & $\geq 7520.000$ & 6387.1 \\
\hline Free OH & 1.000 & NR & NR & & NR & NR & & & NR & NR & & & NR & NR \\
\hline Carbonate & 1.000 & 10490.000 & 8909.7 & $\leq 100,00$ & NR & NR & & & NR & NR & & & $\geq 10490.000$ & 8909.7 \\
\hline TOC & 2.450 & .867 & 7 & 0 & NR & NR & & & NR & NR & & & $\geq 889.333$ & 755.4 \\
\hline Nitrite & 1.000 & 7264.000 & 6169.7 & 0.00 & NR & NR & & & NR & NR & & & $\geq 7264.000$ & 6169.7 \\
\hline Sulfate & 1.000 & 19050.000 & 16180.2 & $\leq 100.00$ & 0.000 & 0.0 & 0.000 & 0,00 & 0,000 & 0.0 & 0.000 & 0.00 & 050.000 & 16180.2 \\
\hline \multicolumn{2}{|l|}{ Total } & \multicolumn{3}{|c|}{$\begin{array}{c}249992.516 \\
62.25 \% \text { total solids } \\
\text { in wash }\end{array}$} & & \multicolumn{3}{|c|}{$\begin{array}{c}118491.291 \\
29.50 \% \text { total solids } \\
\text { in acid }\end{array}$} & \multicolumn{4}{|c|}{$\begin{array}{l}26408.515 \\
6.58 \% \text { total solids } \\
\text { in fusion }\end{array}$} & \multicolumn{2}{|c|}{$\begin{array}{c}98.33 \% \text { mass } \\
\text { balance }\end{array}$} \\
\hline \multicolumn{2}{|l|}{\begin{tabular}{|l} 
Radionuclides \\
\end{tabular}} & $u C i / g$ & Ci/tank & to dissolved & $u C i / g$ & Ci/tank & & $\%$ dissolved & $u C i / g$ & Ci/tank & & \% dissolved & uCi/g & Ci/tank \\
\hline \multirow{4}{*}{\multicolumn{2}{|c|}{$\begin{array}{l}\text { Pu-239,240 } \\
C-14 \\
\text { St-90 } \\
\text { Tc-99 }\end{array}$}} & NR & NR & & NA & & & & 0.080 & 68.0 & & $\leqslant 100.00$ & 0.080 & 68.0 \\
\hline & & 0.005 & 4.2 & $\leq 100.00$ & NA & & & & NR & NR & & & $\geq 0.005$ & 4.2 \\
\hline & & NR & NR & & NA & & & & 4.980 & 4229.8 & & $\leqslant 100.00$ & 4.980 & 4229.8 \\
\hline & & NR & NR & & NA & & & & 0.035 & 30.1 & & $\leqslant 100.00$ & 0.035 & 30.1 \\
\hline \multirow{4}{*}{\multicolumn{2}{|c|}{$\begin{array}{l}A m-241 \\
\text { Co-60 } \\
\text { Cs-137 } \\
1-129\end{array}$}} & NR & NR & & NA & & & & 0.005 & 4.1 & & $\leq 100.00$ & 0.005 & 4.1 \\
\hline & & NR & NR & & NA & & & & $<0.04$ & $<34$ & & & $<0.04$ & $<34$ \\
\hline & & NR & NR & & NA & & & & 19.440 & 16511.4 & & $\leq 100.00$ & 19.440 & 16511.4 \\
\hline & & NR & NR & & NA & & & & $<0.019$ & $<16$ & & & $<0.019$ & $<16$ \\
\hline
\end{tabular}

$1-129$

1. Aluminum oxide factor for acid 2.79 (based on $85 \% \mathrm{Al}(\mathrm{OH}) 3$ and $15 \% \mathrm{AlOOH}$ )

2. Uranium analysis on fused sample only; uranium assumed to dissolve in acid

3. With the exception of $\mathrm{C}-14$, all radionuclide analyses were performed on fused samples

NR - Not Reported

4. Na7F(PO4)2 probably exists in this waste. 


\begin{tabular}{|c|c|c|c|c|c|c|c|c|c|c|c|c|c|c|}
\hline $\begin{array}{l}\text { Density }(\mathrm{g} / \mathrm{mL}) \\
\text { Volume }(\mathrm{L}) \\
\text { Mass (kg) } \\
\text { Solids (ug/g) } \\
\sim \mathrm{pH} \\
\end{array}$ & $\begin{array}{r}1.50 \\
393640 \\
590460 \\
585000 \\
11-12.3 \\
\end{array}$ & & & & & & & & & & & & & \\
\hline Component & $\begin{array}{l}\text { Oxide } \\
\text { Factor }\end{array}$ & $\begin{array}{l}\text { ug/g sludge } \\
\text { (element) }\end{array}$ & $\begin{array}{r}\text { WATER } \\
\text { kg/tank } \\
\text { (element) }\end{array}$ & $\begin{array}{l}\text { \% element } \\
\text { dissolved }\end{array}$ & $\begin{array}{l}\text { ug/g sludge } \\
\text { (element) }\end{array}$ & $\begin{array}{r}\mathrm{kg} / \operatorname{tank} \\
\text { (element) }\end{array}$ & $\begin{array}{l}\text { ug/g sludge } \\
\text { (oxide) }\end{array}$ & $\begin{array}{r}\text { \% element } \\
\text { dissolved }\end{array}$ & $\begin{array}{r}\text { ug/g sludge } \\
\text { (element) }\end{array}$ & \multicolumn{2}{|c|}{$\begin{array}{l}\text { kg/tank ug/g sludge } \\
\text { (oxide) }\end{array}$} & $\begin{array}{l}\text { \% element } \\
\text { dissolved }\end{array}$ & $\begin{array}{c}\text { ug/g sludge } \\
\text { (element) }\end{array}$ & $\begin{array}{l}\text { kg/tank } \\
\text { (element) }\end{array}$ \\
\hline Alurnínum (1) & 1.889 & 564.500 & 333.3 & 3.12 & 13838.500 & 8171.1 & 38609.415 & 76.45 & 3697.500 & 2183.2 & 6984.578 & 20.43 & 18100.500 & 10687.6 \\
\hline Barium & 1.117 & $\angle D L$ & & & 69.950 & 41.3 & 78.134 & 80.49 & 16.950 & 10.0 & 18.933 & 19.51 & 86.900 & 51.3 \\
\hline Bismuth & 1.115 & NR & NR & & NR & NR & & & NR & NR & & & NR & NR \\
\hline Boron & 3.220 & 34.350 & 20.3 & 26.86 & 93.550 & 55.2 & 301.231 & 73.14 & 0.000 & 0.0 & 0.000 & 0.00 & 127.900 & 75.5 \\
\hline Cadmium & 1.142 & $<D L$ & & & 5.500 & 3.2 & 6.281 & 100.00 & 0.000 & 0.0 & 0.000 & 0.00 & 5.500 & 3.2 \\
\hline Calcium & 1.399 & 312.500 & 184.5 & 1.27 & 19989.000 & 11802.7 & 27964.611 & 80.98 & 4383.500 & 2588.3 & 6132.517 & 17.76 & 24685.000 & 14575.5 \\
\hline Chromium & 1.462 & 159.000 & 93.9 & 63.33 & 30.300 & 17.9 & 44.299 & 12.07 & 61.750 & 36.5 & 90.279 & 24.60 & 251.050 & 148.2 \\
\hline Cobalt & 1.407 & $<D L$ & & & $<D L$ & & & & $<D L$ & & & & $<D L$ & \\
\hline Copper: & 1.252 & $<D L$ & & & 11.850 & 7.0 & 14.836 & 20.70 & 45.400 & 26.8 & 56.841 & 79.30 & 57.250 & 33.8 \\
\hline Iron & 1.430 & 1512.000 & 892.8 & 6.19 & 18251.500 & 10776.8 & 26099.645 & 74.71 & 4667.000 & 2755.7 & 6673.810 & 19.10 & 24430.500 & 14425.2 \\
\hline Lead & 1.077 & $\angle D L$ & 0.0 & 0.00 & 1918.150 & 1132.6 & 2065.848 & 87.60 & 271.500 & 160.3 & 292.406 & 12.40 & 2189.650 & 1292.9 \\
\hline Magnesium & 1.658 & 36.650 & 21.6 & 6.51 & 450.450 & 266.0 & 746.846 & 80.07 & 75.500 & 44.6 & 125.179 & 13.42 & 562.600 & 332.2 \\
\hline Manganese & 1.582 & 1.850 & 1.1 & 0.78 & 175.100 & 103.4 & 277.008 & 74.21 & 59.000 & 34.8 & 93.338 & 25.01 & 235.950 & 139.3 \\
\hline Nickel & 1.409 & 895.500 & 528.8 & 5.37 & 13237.500 & 7816.2 & 18651.638 & 79.44 & 2530.000 & 1493.9 & 3564.770 & 15.18 & 16663.000 & 9838.8 \\
\hline Phosphate & 1.000 & 34245.000 & 20220.3 & 38.88 & 40663.500 & 24010.2 & 40663.500 & 46.17 & 13171.500 & 7777.2 & 13171.500 & 14.95 & 88080.000 & 52007.7 \\
\hline Potassium & 1.000 & 542.500 & 320.3 & 86.77 & 82.700 & 48.8 & 82.700 & 13.23 & NA & & & & 625.200 & 369.2 \\
\hline Silicon & 2.139 & 73.100 & 43.2 & 2.87 & 1073.500 & 633.9 & 2296.217 & 42.17 & 1399.000 & 826.1 & 2992.461 & 54.96 & 2545.600 & 1503.1 \\
\hline Silver & 1.074 & $<D L$ & & & $\angle \mathrm{DL}$ & & & & $<\mathrm{DL}$ & & & & $<D L$ & \\
\hline Sodium & 1.000 & 106648.000 & 629714 & 90.52 & 0.000 & 0.0 & 0.000 & 0.00 & 11167.000 & 6593,7 & 11167.000 & 9,48 & 117815.000 & 69565.0 \\
\hline Strontium & 1.183 & 3.650 & 2.2 & 1.15 & 263.500 & 155.6 & 311.721 & 82.71 & 51.450 & 30.4 & 60 & 16.15 & 318.600 & 188.1 \\
\hline Zinc & 1.245 & 5.700 & 3.4 & 1.59 & 254.200 & 150.1 & 316.479 & 71.12 & 97.500 & 57.6 & 121.388 & 27.28 & 357.400 & 211.0 \\
\hline Zirconium & 1.351 & $<D L$ & & & 23.800 & 14.1 & 32.154 & 92.79 & 1.850 & 1.1 & 2.499 & 7.21 & 25.650 & 15.1 \\
\hline Uranium & 1.202 & 2656.000 & 1568.3 & 4.45 & 45343.000 & 267732 & 54502.286 & 76.01 & 11656.500 & 6882.7 & $14011: 113$ & 19.54 & 59655.500 & 35224.2 \\
\hline Nitrate & 1.000 & 75750.000 & 44727.3 & $\leq 100.00$ & NR & NR & & & NR & NR & & & $\geq 75750.000$ & 44727.3 \\
\hline Chloride & 1.000 & 1175.000 & 693.8 & $\leq 100.00$ & NR & NR & & & NR & NR & & & $\geq 1175.000$ & 693.8 \\
\hline Fluoride & 1.000 & 725.000 & 428.1 & $\leq 100.00$ & NR & NR & & & NR & NR & & & $\geq 725.000$ & 428.1 \\
\hline & 1.000 & 1682.500 & 993.4 & 20.03 & NR & NR & & & 6717.500 & 3966.4 & 6717.500 & 79.97 & 8400.000 & 4959.9 \\
\hline Carbonate & 1.000 & 31375.000 & 18525.7 & $\leq 100.00$ & NR & NR & & & NR & NR & & & $\geq 31375.000$ & 18525.7 \\
\hline TOC & 2.450 & 7595.000 & 1830.4 & $\leq 100.00$ & NR & NR & & & NR & NR & & & $\geq 3100.000$ & 1830.4 \\
\hline Nitrite & 1.000 & 57250.000 & 33803.8 & $\leq 100,00$ & NR & NR & & & NR & NR & & & $\geq 57250.000$ & 33803.8 \\
\hline Sulfate & 1.000 & 14650.000 & 8650.2 & & \multirow{2}{*}{\multicolumn{4}{|c|}{ 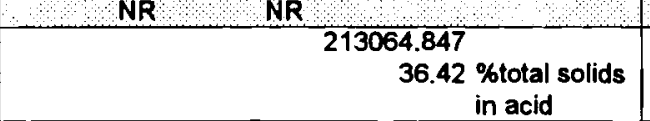 }} & NR & NR & & & 0.000 & 8650.2 \\
\hline \multicolumn{2}{|l|}{ Total } & \multicolumn{3}{|c|}{$\begin{array}{c}337892.800 \\
57.76 \text { \%total solids } \\
\text { in wash }\end{array}$} & & & & & \multicolumn{4}{|c|}{$\begin{array}{c}72276.975 \\
12.36 \% \text { total solids } \\
\text { in fusion }\end{array}$} & \multicolumn{2}{|c|}{$\begin{array}{r}106.54 \% \text { mass } \\
\text { balance }\end{array}$} \\
\hline \multicolumn{2}{|l|}{ Radionuclides } & $\mathrm{uCi} / \mathrm{g}$ & Ci/tank & $\%$ dissolved & $\mathrm{uCi} / \mathrm{g}$ & Ci/tank & & $\%$ dissolved & $\mathrm{uCi} / \mathrm{g}$ & Ci/tank & & \% dissolved & $\mathrm{uCi} / \mathrm{g}$ & Ci/tank \\
\hline $\begin{array}{l}\text { Pu-239,240 } \\
\text { C-14 }\end{array}$ & & $\begin{array}{r}\text { NR } \\
0.00002\end{array}$ & $\begin{array}{l}\text { NR } \\
0.01\end{array}$ & $\leq 100.00$ & $\begin{array}{l}\text { NA } \\
\text { NA }\end{array}$ & & & & $\begin{array}{l}0.107 \\
\text { NR }\end{array}$ & $\begin{array}{c}63.2 \\
\text { NR }\end{array}$ & & $\leq 100.00$ & $\begin{array}{r}0.107 \\
\geq 0.00002\end{array}$ & $\begin{array}{l}63.2 \\
0.01\end{array}$ \\
\hline $5 r-90$ & & NR & NR & & NA & & & & 2004.000 & 1183281.8 & & $\leq 100.00$ & 2004,000 & 1183281.8 \\
\hline TC-99 & & NR & NR & & NA & & & & 0.124 & 72.9 & & $\leq 100.00$ & 0.124 & 72.9 \\
\hline Am-241 & & 0.009 & 5.3 & 264 & NA & & & & 0.333 & 196.3 & & 97.36 & 0.342 & 201.6 \\
\hline Co-60 & & 0.004 & 2.4 & 20.00 & NA & & & & 0.016 & 9.4 & & 80.00 & 0.020 & 11.8 \\
\hline Cs-137 & & 6.160 & 36372 & 0.77 & NA & & & & 789.000 & 4658729 & & 99.23 & 795.160 & 469510.2 \\
\hline $1-129$ & & NR & NR & & NA & & & & NR & NR & & & NR & NR \\
\hline
\end{tabular}

NOTES:

1. Acid soluble oxide factor $2.79(85 \% \mathrm{Al}(\mathrm{OH}) 3,15 \% \mathrm{AlOOH})$

2. Nitrate on water leach only, would not account for nitrate in cancrinite

3. No reported values for $1-129$

NR - Not Reported

NA - No Analysis Performed

$<D L$ - Less than Detection Limit 


\begin{tabular}{|c|c|c|c|c|c|c|c|c|c|c|c|c|c|c|}
\hline \multirow{2}{*}{ 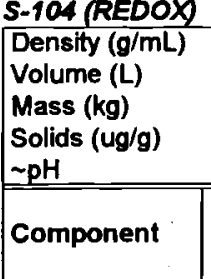 } & \multirow{2}{*}{$\begin{array}{r}1.50 \\
1109005 \\
1663508 \\
652000 \\
13.0 \\
\text { Oxide } \\
\text { Factor }\end{array}$} & \multicolumn{13}{|l|}{ estimated } \\
\hline & & $\begin{array}{r}\text { ug/g sludge } \\
\text { (element) }\end{array}$ & $\begin{array}{l}\text { WATER -- } \\
\text { kg/tank } \\
\text { (element) }\end{array}$ & $\begin{array}{l}\% \text { element } \\
\text { dissolved }\end{array}$ & $\begin{array}{l}\text { ug/g sludge } \\
\text { (element) }\end{array}$ & \multicolumn{2}{|c|}{$\begin{array}{c}\mathrm{kg} / \text { tank } \text { ug/g sludge } \\
\text { (element) }\end{array}$} & $\begin{array}{l}\text { \% element } \\
\text { dissolved }\end{array}$ & $\begin{array}{l}\text { ug/g sludge } \\
\text { (element) }\end{array}$ & \multicolumn{2}{|c|}{$\begin{array}{c}\text { kg/tank ug/g sludge } \\
\text { (element) }\end{array}$} & $\begin{array}{l}\text { \% element } \\
\text { dissolved }\end{array}$ & $\begin{array}{l}\text { ug/g sludge } \\
\text { (element) }\end{array}$ & $\begin{array}{r}\overline{\mathrm{kg} / \operatorname{tank}} \\
\text { (element) }\end{array}$ \\
\hline Aluminum (1) & $1: 889$ & 4731.667 & 7871.2 & 4.01 & 34268.333 & 57005.6 & 95608.650 & 29.04 & 79000.000 & 1314171 & 173800000 & 66.95 & $1: 18000.000$ & 196293.9 \\
\hline Barium & 1.117 & 0.310 & 0.5 & 0.93 & 21.557 & 35.9 & 24.079 & 64.80 & 11.400 & 19.0 & 12.734 & 34.27 & 33.267 & 55.3 \\
\hline $\begin{array}{l}\text { Bismuth } \\
\text { Boron }\end{array}$ & $\begin{array}{l}1.115 \\
3.220\end{array}$ & $\begin{array}{l}\angle D L \\
8.983\end{array}$ & 14.9 & 22.94 & $\begin{array}{l}<D L \\
5.467\end{array}$ & 9.1 & 17.603 & 13.96 & $\begin{array}{r}<D L \\
24.717\end{array}$ & 41.1 & 79.588 & 63.11 & $\begin{array}{l}<D L \\
39.167\end{array}$ & 65.2 \\
\hline Cadmium & 1.142 & $<D L$ & & & $<D L$ & & & & $<\mathrm{DL}$ & & & & $<D L$ & \\
\hline Calcium & 1.399 & 98.683 & 164.2 & 5.16 & 158.983 & 264.5 & 222.418 & 8.31 & 1655.667 & 2754.2 & 2316.278 & 86.53 & 1913.333 & 3182.8 \\
\hline Chromium & 1.462 & 1914.000 & 3184.0 & 44.64 & 2373.333 & 3948.1 & 3469.813 & 55.36 & 0.000 & 0.0 & 0.000 & 0.00 & 4287.333 & 7132.0 \\
\hline Cobalt & 1.407 & 1.200 & 2.0 & 17.82 & 1.383 & 2.3 & 1.946 & 20.54 & 4.150 & 6.9 & 5.839 & 61.63 & 6.733 & 11.2 \\
\hline Copper & 1.252 & 0.850 & 1.4 & 1.64 & 18.800 & 31.3 & 23.538 & 36.31 & 32.133 & 53.5 & 40.231 & 62.05 & 51.783 & 86.1 \\
\hline Iron & 1.430 & 3.317 & 5.5 & 0.13 & 771.350 & 1283.1 & 1103.031 & 31.03 & 1711.550 & 2847.2 & 2447.516 & 68.84 & 2486.217 & 4135.8 \\
\hline Lead & 1.077 & 8.150 & 13.6 & 21.12 & 18.217 & 30.3 & 19.619 & 47.21 & 12.217 & 20.3 & 13.157 & 31.66 & 38.583 & 64.2 \\
\hline Magnesium & 1.658 & 4.483 & 7.5 & 8.39 & 39.500 & 65.7 & 65.491 & 73.88 & 9.483 & 15.8 & 15.723 & 17.74 & 53.467 & 88.9 \\
\hline Manganese & 1.582 & 0.303 & 0.5 & 0.03 & 934.197 & 1554.0 & 1477.899 & 85.34 & 160.167 & 266.4 & 253.384 & 14.63 & 1094.667 & 1821.0 \\
\hline Nickel & 1.409 & 1.667 & 2.8 & 0.03 & 54.817 & 91.2 & 77.237 & 1.12 & 4826.850 & 8029.5 & 6801.032 & 98.84 & 4883.333 & 8123.5 \\
\hline Phosphate & 1.000 & 27.250 & 45.3 & 9.43 & 32.800 & 54.6 & 32.800 & 11.35 & 228.950 & 380.9 & 228.950 & 79.22 & 289.000 & 480.8 \\
\hline Potassium & 1.000 & 252.833 & 420.6 & 84.28 & 47.167 & 78.5 & 47.167 & 15.72 & & 0.0 & 0.000 & 0.00 & 300.000 & 499.1 \\
\hline Silicon & 2.139 & 34.433 & 57.3 & 2.36 & 165.567 & 275.4 & 354.147 & 11.34 & 1260.000 & 2096.0 & 2695.140 & 86.30 & 1460.000 & 2428.7 \\
\hline Silver & 1.074 & $\angle D L$ & & & $<D L$ & & & & $<D L$ & & & & $<D L$ & \\
\hline Sodium & 1.000 & 118250.000 & 196709.8 & 99.09 & 1083.333 & 1802.1 & 1083.333 & 0.91 & 0.000 & 0.0 & 0.000 & 0.00 & 119333.333 & 198511.9 \\
\hline Strontium & 1.183 & 0.635 & 1.1 & 0.15 & 329.698 & 548.5 & 390.033 & 78.78 & 88.167 & 146.7 & 104.301 & 21.07 & 418.500 & 696.2 \\
\hline Zine & 1.245 & 12.383 & 20.6 & 3.81 & 8.567 & 14,3 & 10.665 & 2.63 & 304.250 & 506.1 & 378.791 & 93.56 & 325.200 & 541.0 \\
\hline Zirconium & 1.351 & 1.020 & 1.7 & 0.16 & 643.547 & 1070.5 & 869.432 & 99.84 & 0.000 & 0.0 & 0.000 & 0.00 & 644.567 & 1072.2 \\
\hline Uranlum & 1.202 & NR & NR & & 6616.667 & 11006.9 & 7953.233 & $<100.00$ & 0.000 & 0.0 & 0.000 & 0.00 & 6616.667 & 11006.9 \\
\hline Nitrate & 1.000 & 185666.667 & 308857.9 & $\$ 100.00$ & NR & NR & & & NR & NR & & & $\geq 185666.667$ & 308857.9 \\
\hline Chloride & 1.000 & 3173.333 & 5278.9 & $\leq 100.00$ & NR & NR & & & NR & NR & & & $\geq 3173.333$ & 5278.9 \\
\hline Fluoride & 1.000 & 145.333 & 241.8 & $\leq 100.00$ & NR & NR & & & NR & NR & & & $\geq 145.333$ & 241.8 \\
\hline Free $\mathrm{OH}$ & 1.000 & NR & NR & & NR & NR & & & NR & NR & & & NR & NR \\
\hline Carbonate & 1.000 & 20973.333 & 34889.3 & $\leq 100.00$ & NR & NR & & & NR & NR & & & $\geq 20973.333$ & 34889.3 \\
\hline TOC & 2.450 & 3768.917 & 2559.0 & $\leq 100.00$ & NR & NR & & & NR & NR & & & $\geq 1538.333$ & 2559.0 \\
\hline Nitrite & 1.000 & 20816.667 & 34628.7 & $\leq 100.00$ & NR & NR & & & NR & NR & & & $\geq 20816.667$ & 34628.7 \\
\hline Sulfate & 1.000 & 2365.000 & 3934.2 & 100.00 & 0,000 & 0.0 & 0.000 & 0.00 & 0,000 & 0.0 & 0.000 & 0.00 & 2365.000 & 3934.2 \\
\hline \multicolumn{2}{|l|}{ Total } & \multicolumn{3}{|c|}{$\begin{array}{c}362261.418 \\
55.56 \% \text { \%total solids } \\
\text { in wash }\end{array}$} & \multirow[b]{2}{*}{$\mathbf{C i / g}$} & \multicolumn{3}{|c|}{$\begin{array}{r}112852.134 \\
17.31 \% \\
\text { in }\end{array}$} & \multicolumn{4}{|c|}{$\begin{array}{c}189192.664 \\
29.02 \% \text { total solids } \\
\text { in fusion }\end{array}$} & \multicolumn{2}{|c|}{$\begin{array}{c}101.89 \% \text { mass } \\
\text { balance }\end{array}$} \\
\hline \multicolumn{2}{|l|}{ Radionuclides } & $u C i / g$ & Ci/tank & $\%$ dissolved & & Ci/tank & & \% dissolved & uCi/g & Ci/tank & & $\%$ dissolved & $\mathrm{uCi} / \mathrm{g}$ & Ci/tank \\
\hline Pu-239,240 & & NR & NR & & NA & & & & 0.284 & 473.0 & & $\leq 100.00$ & 0.284 & 473.0 \\
\hline C-14 & & 0.001 & 1.6 & $\leq 100.00$ & NA & & & & NR & NR & & & $\geq 0.001$ & 1.6 \\
\hline Sir 90 & & NR & NR & & NA & & & & 303.333 & 504597.3 & & $\leq 100.00$ & 303.333 & $504597: 3$ \\
\hline Tc-99 & & NR & NR & & NA & & & & 0.025 & 40.8 & & $\leq 100.00$ & 0.025 & 40.8 \\
\hline$A m-241$ & & NR & NR & & NA & & & & 0.118 & 1963 & & $\leq 100.00$ & 0.118 & 1963 \\
\hline Co-60 & & NR & NR & & NA & & & & $<0.033$ & $<55.6$ & & & $<0.033$ & $<55.6$ \\
\hline Cs-137. & & NR & NR & & NA & & & & 63.182 & 105103.2 & & $\leq 100.00$ & 63.182 & 105103.2 \\
\hline $1-129$ & & NR & NR & & NA & & & & 0.013 & 22.3 & & $\leq 100.00$ & 0.013 & 22.3 \\
\hline
\end{tabular}

1. Acid soluble oxide factor $2.79(85 \% \mathrm{Al}(\mathrm{OH}) 3,15 \% \mathrm{AlOOH})$; fusion soluble oxide factor $2.22(\mathrm{~A} / \mathrm{OOH})$

2. Uranium arialysis performed on fused sample only; uranium assumed to be acid soluble

3. Radionuclide analyses on fused sample only, with exception of $C_{-14}$

NR - Not Reported

4. Co-60 value less than 


\begin{tabular}{|c|c|c|c|c|c|c|c|c|c|c|c|c|c|c|}
\hline 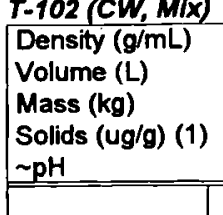 & $\begin{array}{r}1.79 \\
71915 \\
128728 \\
985000 \\
12.0 \\
\end{array}$ & (estimated) & & \multirow[b]{2}{*}{$\begin{array}{l}\text { \% element } \\
\text { dissolved }\end{array}$} & \multirow[b]{2}{*}{$\begin{array}{r}\text { ug/g sludge } \\
\text { (element) }\end{array}$} & \multirow{2}{*}{\multicolumn{2}{|c|}{$\begin{array}{c}\mathrm{kg} / \mathrm{tank} \text { ug/g sludge } \\
\text { (element) }\end{array}$}} & \multirow[b]{2}{*}{$\begin{array}{l}\text { \% element } \\
\text { dissolved }\end{array}$} & \multirow[b]{2}{*}{$\begin{array}{r}\text { ug/g sludge } \\
\text { (element) }\end{array}$} & & \multirow[b]{2}{*}{$\begin{array}{l}\text { \% element } \\
\text { dissolved }\end{array}$} & \multirow[b]{2}{*}{$\begin{array}{c}\text { ug/g sludge } \\
\text { (element) }\end{array}$} & \multirow[b]{2}{*}{$\begin{array}{r}\mathrm{kg} / \operatorname{tank} \\
\text { (element) }\end{array}$} \\
\hline Component & $\begin{array}{l}\text { Oxide } \\
\text { Factor }\end{array}$ & $\begin{array}{r}\text { ug/g sludge } \\
\text { (element) }\end{array}$ & $\begin{array}{r}\text { WATER - } \\
\text { kg/tank } \\
\text { (element) }\end{array}$ & & & & & & & $\begin{array}{r}\mathrm{kg} / \text { tank } \\
\text { (element) }\end{array}$ & $\begin{array}{r}\text { ug/g sludge } \\
\text { (oxide) }\end{array}$ & & & \\
\hline Aluminum (1) & 1.889 & 791.750 & 101.9 & 0.26 & 157322.000 & 202517 & 438928.380 & 52.14 & 143609.750 & 18486.6 & 400671.203 & 47.60 & 301723.500 & 38840.2 \\
\hline Barium & 1.117 & $<2$ & $<0.4$ & & 12.000 & 1.5 & 13.404 & 100.00 & $<111$ & $<24$ & & & 12.000 & 1.5 \\
\hline Bismuth & 1.115 & $<101$ & $<219$ & & $<199$ & $<43$ & & & $\angle D L$ & & & & $<D L$ & \\
\hline Boron & 3.220 & $<4$ & $<0.9$ & & $>0$ & $>0$ & & & $>0$ & $>0$ & & & $>0$ & $>0$ \\
\hline Cadmium & 1.142 & $<1$ & $<0.2$ & & 12.750 & 1.6 & 14.561 & 100.00 & $>0$ & $>0$ & & & 12.750 & 1.6 \\
\hline Calcium & 1.399 & 13.500 & 1.7 & 1.83 & 318.000 & 40.9 & 444.882 & 43.03 & 407.500 & 52.5 & 570.093 & 55.14 & 739.000 & 95.1 \\
\hline $\begin{array}{l}\text { Chromium } \\
\text { Cobalt }\end{array}$ & $\begin{array}{l}1.462 \\
1.407\end{array}$ & $\begin{array}{r}771.750 \\
<2\end{array}$ & $\begin{array}{l}99.3 \\
<0.4\end{array}$ & 98.12 & $\begin{array}{r}0.000 \\
<4\end{array}$ & $\begin{array}{l}0.0 \\
<0.9\end{array}$ & 0.000 & 0.00 & $\begin{array}{r}14.750 \\
<111\end{array}$ & $\begin{array}{r}1.9 \\
<24\end{array}$ & 21.565 & 1.88 & 786.500 & 101.2 \\
\hline $\begin{array}{l}\text { Coban } \\
\text { Copper }\end{array}$ & 1.252 & $<1$ & $<0.2$ & & 17.000 & 2.2 & 21.284 & 29.82 & 40.000 & 5.1 & 50.080 & 70.18 & $\begin{array}{r}<\mathrm{LL} \\
57.000\end{array}$ & 7.3 \\
\hline Iron & 1.430 & 104.333 & 13.4 & 0.52 & 20095.417 & 2586.8 & 28736.446 & 99.48 & 0.000 & 0.0 & 0.000 & 0.00 & 20199.750 & 2600.3 \\
\hline Lead & 1.077 & $<12$ & $<2.6$ & & 421.500 & 54.3 & 453.956 & 100.00 & 0.000 & 0.0 & 0.000 & 0.00 & 421.500 & 54.3 \\
\hline Magnesium & 1.658 & $<20$ & $<4.3$ & & 107.000 & 13.8 & 177.406 & 100.00 & $<D L$ & & & & 107.000 & 13.8 \\
\hline Manganese & 1.582 & 8.000 & 1.0 & 0.82 & 789.250 & 1016 & 1248.594 & 81.12 & 175.750 & 22.6 & 278.037 & 18.06 & 973.000 & 125.3 \\
\hline Nickel & 1.409 & $<6$ & $<1.3$ & & 73.000 & 9.4 & 102.857 & 100.00 & NA & & & & 73.000 & 9.4 \\
\hline Phosphate & 1.000 & 1239.750 & 159.6 & 26.95 & 553.500 & 713 & 553.500 & 1203 & 2807.250 & 361.4 & 2807.250 & 61.02 & 4600,500 & 592.2 \\
\hline Potassium & 1.000 & $<202$ & $<43.8$ & & $<399$ & $<86.5$ & & & NA & & & & $<D L$ & \\
\hline Silticon & 2.139 & 46.000 & 5.9 & 1.42 & 807000 & 103.9 & 1726.173 & 24.91 & 2386.500 & 307.2 & 5104.724 & 73.87 & 3239.500 & 417.0 \\
\hline Silver & 1.074 & & $<0.4$ & & 15.000 & 1.9 & 16.110 & 10.31 & 130.500 & 16.8 & 140.157 & 89.69 & 145.500 & 18.7 \\
\hline Sodium & 1.000 & 28813.750 & 3709,1 & 100.00 & 0.000 & 0.0 & 0.000 & 0.00 & 0.000 & 0.0 & 0.000 & 0.00 & 28813.750 & 3709.1 \\
\hline Strontium & 1.183 & $<1$ & $<0.2$ & & 17.750 & 2.3 & 20.998 & 100.00 & $<55$ & $<12$ & & & 17.750 & 2.3 \\
\hline Zinc & 1.245 & $<4$ & $<09$ & & 112.500 & 14.5 & 140.063 & 49.78 & 113.500 & 14.6 & 141,308 & 50.22 & 226.000 & 29.1 \\
\hline Zirconium & 1.351 & $<2$ & $<0.4$ & & 41.750 & 5.4 & 56.404 & 100.00 & $<111$ & $<24$ & & & 41.750 & 5.4 \\
\hline Uranium & 1.202 & $<403$ & $<873$ & & 734.000 & 945 & 882.268 & 100.00 & $<D L$ & & & & 734,000 & 94.5 \\
\hline Nitrate & 1.000 & 35000.000 & 4505.5 & $\leq 100.00$ & NR & NR & & & NR & NR & & & $\geq 35000.000$ & 4505.5 \\
\hline Chloride & 1.000 & 300.000 & 38.6 & $\$ 10000$ & NR & NR & & & NR & NR & & & $\geq 300.000$ & 38.6 \\
\hline Fluoride & 1.000 & 220.000 & 28.3 & $\leq 100.00$ & NR & NR & & & NR & NR & & & $\geq 220.000$ & 28.3 \\
\hline Free $\mathrm{OH}$ & 1.000 & NR & NR & & NR & NR & & & NR & NR & & & NR & NR \\
\hline Carbonate & 1.000 & 12350.000 & 1589.8 & $\leq 100.00$ & 0.000 & 0.0 & 0.000 & 0.00 & NR & NR & & & $\geq 12350.000$ & 1589.8 \\
\hline TOC & 2.450 & 1604750 & 843 & $\leq 100.00$ & NR & NR & & & NR & NR & & & $\geq 655,000$ & 84.3 \\
\hline Nitrite & 1.000 & 8000.000 & 1029.8 & $\leq 100.00$ & NR & NR & & & NR & NR & & & $\geq 8000.000$ & 1029.8 \\
\hline Sulfate & 1,000 & 1670.000 & 215.0 & $\$ 100.00$ & NR & NR & & & NR & NR & & & $\geq 1670.000$ & 215.0 \\
\hline Total & & $\begin{array}{r}90933.583 \\
9.23 \\
\end{array}$ & $\begin{array}{l}\text { total solids } \\
\text { wash }\end{array}$ & & & & $\begin{array}{r}473537.284 \\
48.07\end{array}$ & $\begin{array}{l}\text { \%total solids } \\
\text { in acid }\end{array}$ & & & $\begin{array}{r}409784.414 \\
41.60\end{array}$ & $\begin{array}{l}\text { \%total solids } \\
\text { in fusion }\end{array}$ & 98.91 & $\begin{array}{l}\% \text { mass } \\
\text { balance }\end{array}$ \\
\hline Radionuclides & & $\mathrm{uCi} / \mathrm{g}$ & CI/tank & $\%$ dissolved & $\mathrm{uCi} / \mathrm{g}$ & Ci/tank & & \% dissolved & uCi/g & Ci/tank & & \% dissolved & $\mathrm{uCi} / \mathrm{g}$ & Ci/tank \\
\hline$P u-239,240$ & & NR & NR & & NA & & & & 0.061 & 79 & & $\leqslant 100.00$ & 0.061 & 7.9 \\
\hline C-14 & & 0.031 & 4.0 & $\leq 100.00$ & NA & & & & NR & NR & & & $\geq 0.031$ & 4.0 \\
\hline Sr-90 & & NR & NR & & NA & & & & 238.000 & 30637.2 & & $\leq 100.00$ & 238.000 & 30637.2 \\
\hline Tc-99 & & NR & NR & & NA & & & & 0.018 & 2.3 & & $\leqslant 100.00$ & 0.018 & 2.3 \\
\hline Am-241 & & NR & NR & & NA & & & & 0.257 & 33.0 & & $\leqslant 100.00$ & 0.257 & 33.0 \\
\hline $\mathrm{Co}-60$ & & 0.001 & 0.1 & 3.06 & NA & & & & 0.026948 & 3.5 & & 96.94 & 0.028 & 3.6 \\
\hline Cs-137 & & 26.400 & 3398.4 & 83.02 & NA & & & & 5.400 & 695.1 & & 16.98 & 31.800 & 4093.5 \\
\hline $1-129$ & & NR & NR & & NA & & & & NR & NR & & & NR & NR \\
\hline
\end{tabular}

NOTES

1. \% Solids as received was $723,000 \mathrm{ug} / \mathrm{g}$ sludge; however, samples were store in hot cell for four months prior to analysis. \% Solids was determined to be 985,000 ug/g at time of analysis.

2. Oxide factor $2.79(85 \% \mathrm{Al}(\mathrm{OH}) 3,15 \% \mathrm{AOOH}))$ 


\begin{tabular}{|c|c|c|c|c|c|c|c|c|c|c|c|c|c|c|}
\hline $\begin{array}{l}\text { Density (g/mL) } \\
\text { Volume (L) } \\
\text { Mass (kg) } \\
\text { Solids (ug/g) } \\
\sim \text { pH }\end{array}$ & $\begin{array}{r}1.15 \\
1672970 \\
1923916 \\
293500 \\
10.0 \\
\end{array}$ & estimated & & & & & & & & & & & & \\
\hline Component & $\begin{array}{l}\text { Oxide } \\
\text { Factor }\end{array}$ & $\begin{array}{r}\text { ug/g sludge } \\
\text { (element) }\end{array}$ & $\begin{array}{r}\text { WATER - } \\
\text { kg/tank } \\
\text { (element) }\end{array}$ & $\begin{array}{l}\text { \% element } \\
\text { dissolved }\end{array}$ & $\begin{array}{r}\text { ug/g sludge } \\
\text { (element) }\end{array}$ & \multicolumn{2}{|c|}{$\begin{array}{l}\mathrm{kg} / \text { tank ug/g sludge } \\
\text { (element) } \\
\text { (oxide) }\end{array}$} & $\begin{array}{l}\text { \% element } \\
\text { dissolved }\end{array}$ & $\begin{array}{l}\text { ug/g sludge } \\
\text { (element) }\end{array}$ & \multicolumn{2}{|c|}{$\begin{array}{r}\mathrm{kg} / \operatorname{tank} \mathrm{ug} / \mathrm{g} \text { sludge } \\
\text { (element) }\end{array}$} & $\begin{array}{l}\text { \% element } \\
\text { dissolved }\end{array}$ & $\begin{array}{l}\text { ug/g sludge } \\
\text { (element) }\end{array}$ & $\begin{array}{l}\overline{\mathrm{kg} / \mathrm{tank}} \\
\text { (element) }\end{array}$ \\
\hline Aluminum & 1.889 & 155.000 & 298.2 & 0.98 & 16045.000 & 30869.2 & 30309.005 & $\begin{array}{r}99.04 \\
\end{array}$ & 0.000 & 0.0 & 0.000 & 0.00 & 16200.000 & 31167.4 \\
\hline Barium & 1.117 & 0.308 & 0.6 & 3.49 & 7.468 & 14.4 & 8.341 & 84.86 & 1.025 & 2.0 & 1.145 & 11.65 & 8.800 & 16.9 \\
\hline Bismuth & 1.115 & 166.750 & 320.8 & 0.88 & 18708.250 & 35993.1 & 20859.699 & 99.12 & 0.000 & 0.0 & 0.000 & 0.00 & 18875.000 & 36313.9 \\
\hline Boron & 3.220 & 11.600 & 22.3 & 83.15 & 2.350 & 4.5 & 7.567 & 16.85 & $<D L$ & & & & 13.950 & 26.8 \\
\hline Cadmium & 1.142 & $<D L$ & & & 1725 & 3.3 & 1.970 & 31.22 & 3.800 & 7.3 & 4.340 & 68.78 & 5.525 & 10.6 \\
\hline Calcium & 1.399 & 183.500 & 353.0 & 27.65 & 98.250 & 189.0 & 137.452 & 14.80 & 382.000 & 734.9 & 534.418 & 57.55 & 663.750 & 1277.0 \\
\hline Chromium & 1.462 & 144750 & 278.5 & 15.80 & 771,250 & 1483.8 & 1127.568 & 84.20 & 0.000 & 0.0 & 0.000 & 0.00 & 916.000 & 1762.3 \\
\hline Cobalt & 1.407 & $\angle D L$ & & & 1.625 & 3.1 & 2.286 & 14.84 & 9.325 & 17.9 & 13.120 & 85.16 & 10.950 & 21.1 \\
\hline Copper & 1.252 & $<D L$ & & & 12.675 & 24.4 & 15.869 & 27.01 & 34.250 & 65.9 & 42.881 & 72.99 & 46.925 & 90.3 \\
\hline Iron & 1.430 & 79.550 & 153.0 & 0.88 & 8935.450 & 17191.1 & 12777.694 & 99.12 & 0.000 & 0.0 & 0.000 & 0.00 & 9015.000 & 17344.1 \\
\hline Lead & 1.077 & $<D L$ & & & 61.175 & 1177 & 65.885 & 100.00 & 0.000 & 0.0 & 0.000 & 0.00 & 61.175 & 117.7 \\
\hline Magnesium & 1.658 & 6.425 & 12.4 & 4.03 & 97.550 & 187.7 & 161.738 & 61.16 & 55.525 & 106.8 & 92.060 & 34.81 & 159.500 & 306.9 \\
\hline Manganese & 1.582 & 0.410 & 0.8 & 0.69 & 31.190 & 60,0 & 49.343 & 52.33 & 28.000 & 53.9 & 44.296 & 46.98 & 59.600 & 114.7 \\
\hline Nickel & 1.409 & $<D L$ & & & 12.675 & 24.4 & 17.859 & 100.00 & NA & & & & 12.675 & 24.4 \\
\hline Phosphate & 1.000 & 19215.000 & 36968.0 & 25.98 & 52710.000 & 101409.6 & 52710.000 & 71.28 & 2025.000 & 3895.9 & 2025.000 & 2.74 & 73950.000 & 142273.6 \\
\hline Potassium & 1.000 & $\mathbf{3 7 . 3 5 0}$ & 71.9 & 43.59 & 48.325 & 93.0 & 48.325 & 56.41 & NA & & & & 85.675 & 164.8 \\
\hline Silicon & 2.139 & 172.000 & 330.9 & 2.67 & 851.000 & 1637.3 & 1820.289 & 13.21 & 5417.000 & 10421.9 & 11586.963 & $84: 11$ & 6440.000 & 12390.0 \\
\hline Silver & 1.074 & $<D L$ & & & $<D L$ & & & & $<D L$ & & & & $<D L$ & \\
\hline Sodium & 1.000 & 46500.000 & 894621 & 72.12 & 17975.000 & 34582.4 & 17975.000 & 27.88 & 0.000 & 0.0 & 0.000 & 0.00 & 64475.000 & 124044.5 \\
\hline Strontium & 1.183 & 1.230 & & 1.25 & 97.495 & 187.6 & 115.337 & 98.75 & 0.000 & 0.0 & 0.000 & 0.00 & 98.725 & 189.9 \\
\hline Zinc & 1,245 & 4.325 & 8.3 & 2.84 & 20.575 & 39.6 & 25.616 & 13.53 & 127.175 & 244.7 & 158.333 & 83.63 & 152.075 & 292.6 \\
\hline Zirconium & 1.351 & 2.550 & 4.9 & 3.69 & 66.525 & 128.0 & 89.875 & 96.31 & 0.000 & 0.0 & 0.000 & $0 . \infty$ & 69.075 & 132.9 \\
\hline Uranlum (i) & 1.202 & NR & NR & & 866.750 & 1667.6 & 1041.834 & $<100,00$ & 0.000 & 0.0 & 0.000 & 0.00 & 866.750 & 1667.6 \\
\hline Nitrate & 1.000 & 58025.000 & 111635.2 & $\leq 100.00$ & NR & NR & & & NR & NR & & & $\geq 58025.000$ & 111635.2 \\
\hline Chloride & 1,000 & 670.250 & 1289.5 & $\leq 100.00$ & NR & NR & & & NR & NR & & & $\geq 670.250$ & 1289.5 \\
\hline Fluoride & 1.000 & 8582.500 & 16512.0 & $\leq 100.00$ & NR & NR & & & NR & NR & & & $\geq 8582.500$ & 16512.0 \\
\hline Free OH & 1.000 & NR & NR & & NR & NR & & & NR & NR & & & NR & NR \\
\hline Carbonate (3) & 1.000 & $<2750$ & $<5330$ & & NR & NR & & & NR & NR & & & $<2750$ & $<5330$ \\
\hline$T O C$ (3) & 2.450 & $<1220$ & $<969$ & & NR & NR & & & NR & NR & & & $<1220$ & $<969$ \\
\hline Nitrite & 1.000 & 4067.500 & 7825.5 & $\leq 100.00$ & NR & NR & & & NR & NR & & & $\geq 4067.500$ & 7825.5 \\
\hline Sulfate & 1000 & & 7421.5 & 99.29 & \multirow[t]{2}{*}{27.500} & \multicolumn{2}{|c|}{$\frac{21.000}{139396.050}$} & 0.71 & 0.000 & 0.0 & & & 3885,000 & 7474.4 \\
\hline \multicolumn{2}{|l|}{ Total } & \multicolumn{3}{|c|}{$\begin{array}{c}141883.498 \\
48.34 \% \text { total solids } \\
\text { in wash }\end{array}$} & & & $\begin{array}{r}139396.050 \\
47.49\end{array}$ & $\begin{array}{l}\text { \%total solids } \\
\text { in acid }\end{array}$ & \multicolumn{4}{|c|}{14502.556} & \multicolumn{2}{|c|}{$\begin{array}{c}100.78 \% \text { mass } \\
\text { balance }\end{array}$} \\
\hline \multicolumn{2}{|l|}{ Radionuclides } & $\mathbf{u C i} / \mathrm{g}$ & Ci/tank & 6 dissolved & $\mathbf{u C i} / \mathbf{g}$ & Ci/tank & & \% dissolved & uCi/g & Ci/tank & & \% dissolved & uC̄i/g & Ci/tank \\
\hline & NA & & & NA & & & & $<0.018$ & $<34.9$ & & & $<0.018$ & $<34.9$ \\
\hline C-14 & & $\angle D L$ & & & NA & & & & NR & NR & & & $\geq 0.000$ & $\geq 0.0$ \\
\hline Sr 90 & & NA. & & & NA & & & & 2.608 & 5016.6 & & $\leq 100.00$ & 2.608 & 5016.6 \\
\hline Tc-99 & & NA & & & NA & & & & 0.001 & 1.2 & & $\leq 100.00$ & 0.001 & 1.2 \\
\hline Am-241 & & NA & & & NA & & & & 0.017 & 32.5 & & $\leq 100.00$ & 0.017 & 32.5 \\
\hline Co-60 & & NA & & & NA & & & & $<0.0003$ & $<0.6$ & & & $<0.0003$ & $<0.6$ \\
\hline Cs -137 & & NA & & & NA & & & & $<0.2$ & $<386$ & & & $<0.2$ & $<386$ \\
\hline $1-129$ & & NA & & & NA & & & & $<0.020$ & $<38.7$ & & & $<0.02$ & $<38.7$ \\
\hline
\end{tabular}

1. Uranium analysis performed on fused sample only; uranium assumed to be acid soluble

2. Radionuclide analyses on fused sample only, with exception of $\mathrm{C}-14$

NR - Not Reported

NA - No Analysis Performed

4. Cs-137, Co-60, $1-129$ and $P u 239,240$ values are less than

Letion 


\begin{tabular}{|c|c|c|c|c|c|c|c|c|c|c|c|c|c|c|}
\hline $\begin{array}{l}\text { Density }(\mathrm{g} / \mathrm{mL}) \\
\text { Volume }(\mathrm{L}) \\
\text { Mass (kg) } \\
\text { Solids (ug/g) } \\
\sim \text { pH }\end{array}$ & $\begin{array}{r}1.50 \\
647235 \\
970853 \\
501750 \\
11.500\end{array}$ & & & & & & & & & & & & & \\
\hline Component & $\begin{array}{l}\text { Oxide } \\
\text { Factor }\end{array}$ & $\begin{array}{r}\text { ug/g sludge } \\
\text { (element) }\end{array}$ & $\begin{array}{r}\text { WATER - } \\
\text { kg/tank } \\
\text { (element) }\end{array}$ & $\begin{array}{l}\text { \% element } \\
\text { dissolved }\end{array}$ & $\begin{array}{r}\text { ug/g sludge } \\
\text { (element) }\end{array}$ & $\begin{array}{r}\mathrm{kg} / \operatorname{tank} \\
\text { (element) }\end{array}$ & $\begin{array}{l}\text { CID } \\
\text { ug/g sludge } \\
\text { (oxide) }\end{array}$ & $\begin{array}{l}\text { \% element } \\
\text { dissolved }\end{array}$ & $\begin{array}{l}\text { ug/g sludge } \\
\text { (element) }\end{array}$ & $\begin{array}{r}\text { kg/tank } \\
\text { (element) }\end{array}$ & $\begin{array}{l}\text { SION } \\
\text { ug/g sludge } \\
\text { (oxide) }\end{array}$ & $\begin{array}{l}\text { \% element } \\
\text { dissolved }\end{array}$ & $\begin{array}{l}\text { ug/g sludge } \\
\text { (element) }\end{array}$ & $\begin{array}{r}\text { kg/tank } \\
\text { (element) }\end{array}$ \\
\hline Aluminum(1) & 1.889 & 651.000 & 632.0 & 3.98 & 13719.000 & 13319.1 & 38276.010 & 83.96 & 1970.000 & 1912.6 & $\mathbf{3 7 2 1 . 3 3 0}$ & 12.06 & 16340.000 & 15863.7 \\
\hline Barium & 1.117 & NR & NR & & NR & NR & & & NR & NR & & & NR & \\
\hline Bismuth & 1.115 & 243.375 & 236.3 & 2.03 & 10611.625 & 10302.3 & 11831.962 & 88.45 & 1142.500 & 1109.2 & 1273.888 & 9.52 & 11997.500 & 11647.8 \\
\hline $\begin{array}{l}\text { Boron } \\
\text { Cadmium }\end{array}$ & $\begin{array}{l}3.220 \\
1.142\end{array}$ & $\begin{array}{r}12.970 \\
\text { NR }\end{array}$ & $\begin{array}{l}12.6 \\
\text { NR }\end{array}$ & 100.00 & $\begin{array}{r}0.000 \\
\text { NR }\end{array}$ & $\begin{array}{l}0.0 \\
\text { NR }\end{array}$ & 0.000 & 0.00 & $\begin{array}{r}0.000 \\
N R\end{array}$ & $\begin{array}{l}0.0 \\
\text { NR }\end{array}$ & 0.000 & 0.00 & 12.970 & 12.6 \\
\hline Calcium & 1.399 & 270.500 & 262.6 & 35.57 & 452.000 & 438.8 & 632.348 & 59.43 & 38.000 & 36.9 & 53.162 & 5.00 & $\begin{array}{r}\text { NR } \\
760.500\end{array}$ & 738.3 \\
\hline Chromium & 1.462 & 210.750 & 204.6 & 58.54 & 143.000 & 138.8 & 209.066 & 39.72 & 6.250 & 6.1 & 9.138 & 1.74 & 360.000 & 349.5 \\
\hline Cobalt & 1.407 & NR & NR & & NR & NR & & & NR & 0.0 & & & NR & \\
\hline Copper & 1.252 & NR & NR & & NR & NR & & & NR & 0.0 & & & NR & \\
\hline Iron & 1.430 & 355.500 & 345.1 & 1.13 & 31119.500 & 30212.4 & 44500.885 & 98.87 & 0.000 & 0.0 & 0.000 & 0.00 & 31475.000 & 30557.6 \\
\hline Lea & 1.077 & 500 & 40.3 & 5,21 & 754.750 & 732.8 & 366 & 94.79 & 0.000 & 0.0 & 0.000 & 0.00 & 250 & 773.0 \\
\hline $\begin{array}{l}\text { Magnesium } \\
\text { Manoanese }\end{array}$ & $\begin{array}{l}1.658 \\
1.582\end{array}$ & $\begin{array}{r}31.950 \\
2558\end{array}$ & $\begin{array}{r}31.0 \\
25\end{array}$ & $\begin{array}{r}14.12 \\
1.15\end{array}$ & $\begin{array}{l}181.550 \\
218.943\end{array}$ & $\begin{array}{l}176.3 \\
2126\end{array}$ & $\begin{array}{l}301.010 \\
346.367\end{array}$ & $\begin{array}{l}80.24 \\
98.85\end{array}$ & $\begin{array}{r}12.750 \\
0.000\end{array}$ & $\begin{array}{r}12.4 \\
0.0\end{array}$ & $\begin{array}{r}21.140 \\
0.000\end{array}$ & $\begin{array}{l}5.64 \\
0.00\end{array}$ & $\begin{array}{l}250 \\
500\end{array}$ & $\begin{array}{l}219.7 \\
215.0\end{array}$ \\
\hline Nickel & 1.409 & 8.843 & 8.6 & 0.34 & 283 & 274.9 & 398.969 & 10.74 & 2345.500 & 2277.1 & 3304.810 & 88.93 & .500 & 2560.6 \\
\hline Phosphate & 1.000 & 71175.000 & 69100.4 & 73.97 & 18825.000 & 18276.3 & 18825.000 & 19.56 & 6225.000 & 6043.6 & 6225.000 & 6.47 & 95225.000 & 93420.3 \\
\hline Potassium & 1.000 & 315.500 & 306.3 & 100.00 & 0.000 & 0.0 & 0.000 & 0.00 & NA & & & & 315.500 & 306.3 \\
\hline Silicon & 2.139 & 1980.600 & 1922.9 & 32.70 & 0.000 & 0.0 & 0.000 & 0.00 & 4076.900 & 3958.1 & 8720.489 & 67.30 & 6057.500 & 5880.9 \\
\hline Silver & 1.074 & NR & NR & & NR & NR & & & NR & NR & & & NR & \\
\hline Sodium & 1.000 & 107900.000 & 104755.0 & 82.84 & 22350,000 & 21698.6 & 22350.000 & 17.16 & 0.000 & 0.0 & 0.000 & 0.00 & 130250.000 & 126453.5 \\
\hline Strontium & $\begin{array}{l}1.183 \\
1245\end{array}$ & 5.530 & $\begin{array}{l}5.4 \\
\text { NR }\end{array}$ & 0.57 & $\begin{array}{r}956.720 \\
\text { NR }\end{array}$ & $\begin{array}{r}928.8 \\
\text { NR }\end{array}$ & 1131.800 & 99.43 & $\begin{array}{r}0.000 \\
\text { NR }\end{array}$ & 0.0 & 0.000 & 0.00 & 962.250 & 934.2 \\
\hline $\begin{array}{l}\text { Zinc } \\
\text { Zirconium }\end{array}$ & $\begin{array}{l}1.245 \\
1.351\end{array}$ & $\begin{array}{l}\mathrm{NR} \\
6.323\end{array}$ & $\begin{array}{l}N R \\
6.1\end{array}$ & 6.79 & $\begin{array}{r}\text { NR } \\
65.228\end{array}$ & $\begin{array}{r}\text { NR } \\
63.3\end{array}$ & 88.122 & 70.01 & $\begin{array}{r}\text { NR } \\
21.625\end{array}$ & $\begin{array}{l}\text { NR } \\
21.0\end{array}$ & 29.215 & 23.21 & $\begin{array}{r}\text { NR } \\
93175\end{array}$ & 905 \\
\hline Uranium & 1.202 & NR & NR & & 25425.000 & 24683.9 & 30560.850 & 100.00 & 0.000 & 0.0 & 0.000 & 0.00 & 254 & 24683.9 \\
\hline Nitrate & 1.000 & 74525.000 & 72352.8 & $\leq 100.00$ & NR & NR & & & NR & NR & & & $\geq 74$ & 52.8 \\
\hline Chloride & 1000 & 540.500 & 524.7 & $\leq 10000$ & NR & NR & & & NR & NR & & & $\geq 540.500$ & 524.7 \\
\hline Fluoride & 1.000 & 11412.500 & 11079.9 & $\leq 100.00$ & NR & NR & & & NR & NR & & & $\geq 11412.500$ & 11079.9 \\
\hline & 1.000 & 76.075 & 73.9 & $\leq 100.00$ & NR & NR & & & NR & NR & & & $\geq 76.075$ & 73.9 \\
\hline Carbonate & 1.000 & 4225.000 & 4101.9 & $\leq 100.00$ & NR & NR & & & NR & NR & & & $\geq 4225.000$ & 4101.9 \\
\hline TOC & 2.450 & 4158.875 & 1648.0 & $\leq 100.00$ & NR & NR & & & NR & NR & & & $\geq 1697.500$ & 1648.0 \\
\hline Nitrite & 1.000 & 11717.500 & 6.0 & $\leq 100.00$ & NR & NR & & & NR & NR & & & $\geq 11717.500$ & 11376.0 \\
\hline Sulfate & 1.000 & 000 & 0.5 & & 0.000 & 0.0 & 0.000 & 0,00 & 0.000 & 0.0 & 0.000 & 0.00 & 10620,000 & 10310.5 \\
\hline Total & & $\begin{array}{r}300487.348 \\
59.89\end{array}$ & solids & & & & $\begin{array}{r}170265.255 \\
33.93 \\
\end{array}$ & solids & & & $\begin{array}{r}23358.170 \\
4.66 \\
\end{array}$ & olids & $98.48 \%$ & $\begin{array}{l}\% \text { mass } \\
\text { balance }\end{array}$ \\
\hline Radi & & $\mathrm{uCi} / \mathrm{g}$ & Ci/tank & $\%$ dissolved & uCi/g & Ci/tank & & \% dissolved & $u \overline{\mathbf{C i}} / \mathbf{g}$ & Ci/tank & & \% dissolved & $\mathrm{uCi} / \mathrm{g}$ & Ci/tank \\
\hline $\mathrm{Pu} 239,240$ & & NR & NR & & NA & & & & 0.152 & 147.8 & & $\leq 100.00$ & 0.152 & 147.8 \\
\hline C-14 & & 0.000144 & 0.1 & $\leq 100.00$ & NA & & & & NR & NR & & & $\geq 0.000144$ & \\
\hline Sr-90 & & NR & NR & & NA & & & & 108.700 & 105531.7 & & 0.00 & 108.700 & 105531.7 \\
\hline TC-99 & & NR & NR & & NA & & & & 0.051 & 49.0 & & $\leq 100.00$ & 0.051 & 49.0 \\
\hline Am-241 & & NR & NR & & NA & & & & 0.014 & 13.6 & & $\$ 100.00$ & & 13.6 \\
\hline $\mathrm{Co}-60$ & & 0.005 & 5.0 & 35.76 & NA & & & & 0.009 & 9.1 & & & 0.015 & 14.1 \\
\hline Cs-137 & & 9.245 & 8975.5 & 43.01 & NA & & & & 12.250 & 11892.9 & & 56.99 & 21.495 & 20868.5 \\
\hline $1-129$ & & NR & NR & & NA & & & & NR & NR & & & NR & NR \\
\hline
\end{tabular}

1. Aluminum oxide factor for acid 2.79 [based on $85 \% \mathrm{Al}(\mathrm{OH}) 3$ and $15 \% \mathrm{AlOOH}$ ]

2. Nitrate on water leach only, would not account for any nitrate associated with cancrinite

3. Sulfur analyses were performed on water, acid, and fusion analyses

NR - Not Reported

4. Co-60 value is less than 
T-111 (BIPO4 2C, BIPO4 224)

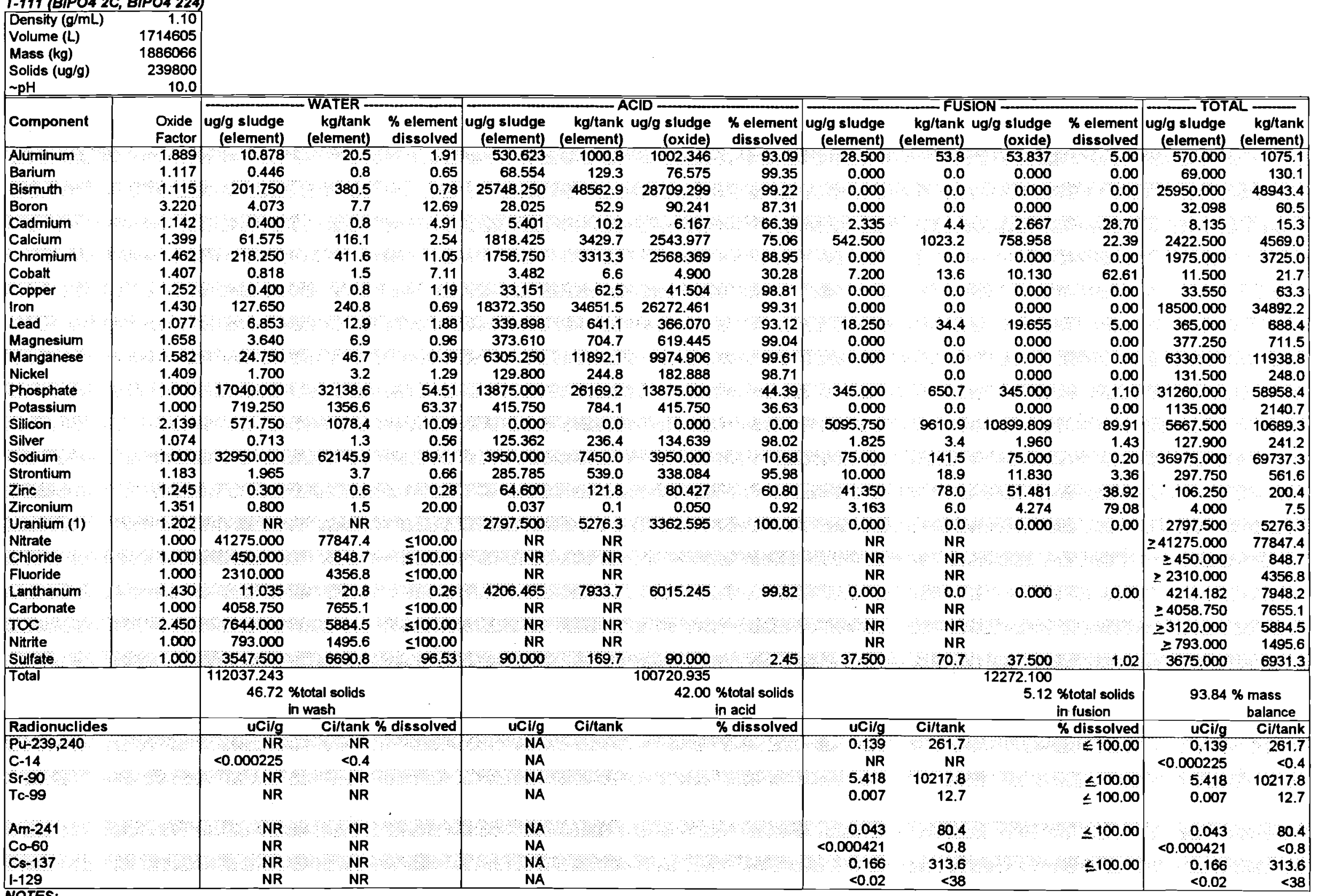




\section{TY-101 (BIPO4 1C, EB)}

\begin{tabular}{|lr|}
\hline Density (g/mL) & 1.64 \\
Volume (L) & 446630 \\
Mass (kg) & 732473 \\
Solids (ug/g) & 565000 \\
\hline
\end{tabular}

$\sim \mathrm{pH} \quad$ NR (drainable liquor $\mathrm{pH} 9.9$ )

Conteren

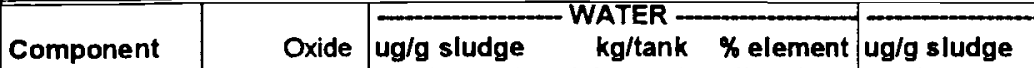
Factor (element)

Aluminum

Bismuth

Con

Cadmium

Chromium

Cobalt

Coppe

Iron

Lead

Manganese

Nickel

Phosphate

N Potassil

Silicon

Silver
Sodium

Strontium

Zine

Zirconium

Uranilum

Nitrate

Chloride

Fluoride

Free $\mathrm{OH}$.

TOC

Nitrite 1.889

(element) (element) dissolved (element)

ACID - w sludge \% element ug/g sludge

FUSION

dissolved (ement) (element) (oxide) dissolved

\begin{tabular}{r|rrrr|rrrrrr} 
(element) & (element) & (oxide) & dissolved & (element) & (element) & (oxide) & dissolved & (element) & (element) \\
0.13 & 13400.000 & 9815.1 & 25312.600 & 46.14 & 15600.000 & 11426.6 & 29468.400 & 53.72 & 29039.000 & 21270.3
\end{tabular}

\begin{tabular}{rrrr|rrrrr}
1.117 & 0.392 & 0.3 & 0.02 & 16.700 & 12.2 & 18.654 & 0.87 & 1910.000
\end{tabular}

\begin{tabular}{r|r}
53.72 & 29039.000 \\
99.11 & 1927.092
\end{tabular}

$\begin{array}{rr}1399.0 & 2133.470 \\ 525.2 & 799.455\end{array}$

\begin{tabular}{r|r}
99.11 & 1927.092 \\
2.63 & 27225.630 \\
\hline
\end{tabular}

21270.3

$\begin{array}{rrrrr}12.2 & 18.654 & 0.87 & 1910.000\end{array}$

525.2
NR

$\begin{array}{rr}8.630 & 6.3 \\ \text { NR } & \text { NR }\end{array}$

3.220
NR
1.950

NR
1.4
NR

0.0326500 .000

NR 29547.500

NR

1.4
NR
310.6

1.399

424.000

18.46 $\quad 3.140$

23
NR

3.586

29.57

NR
5.520

NR
4.0
NR

6.304

\begin{tabular}{r|r}
51.98 & NR \\
10.620
\end{tabular}

19942.0

1.462
1.407

NR
NR

NR

53.100
6.360

$5.05 \quad 500.000$

$366.2 \quad 731.000$

1.430

NR
38.9

NR NR

\begin{tabular}{r|rr}
1.430 & 53.100 & 38.9 \\
1.077 & 6.360 & 4.7
\end{tabular}

$\begin{array}{rrr}1.658 & \text { NR } & \text { NR } \\ 1.582 & 15.700 & 115\end{array}$

15.700

11.5
56.2

$0.13 \quad 13000.000$

NR
9522.18590 .000

$5.95 \quad 7480.000$
NR

NR
5478.9
NR

10935.760

\begin{tabular}{r|rrr}
2.84 & 157.000 & 115.0 & 169.089
\end{tabular}

NR

NR0.0 38753.000

\begin{tabular}{r|r}
32.38 & 27100.000 \\
70.04 & 60.800
\end{tabular}

$28.1 \quad 60.591$

\begin{tabular}{r|r} 
NR \\
6.86 & 504.000
\end{tabular}

504.000

44.5
NR

65.482

NR
$89.01 \quad 8404.000$

\begin{tabular}{r|rr}
1.409 & 76.700 & 56.2 \\
1.000 & 16170.000 & 11844.1
\end{tabular}

\begin{tabular}{r|rr}
1.000 & NR & NR \\
2.139 & 322.000 & 235.9
\end{tabular}

322.000

235.9
0.6

$151 \quad 38.300$

$655.6 \quad 1261.055$

17.58

369.2
3017.8

797.328
5805.080

5805.080

$\begin{array}{ll}553.7 & 756.000\end{array}$

\begin{tabular}{r|r}
21.78 & 57300.000 \\
NR
\end{tabular}

$1970.7 \quad 57300.000$

\begin{tabular}{r|rr}
1.000 & 75000.000 & 54935.5 \\
1.183 & NR & NR
\end{tabular}

$\begin{array}{llll}0.83 & 657.000 & 481.2 & 1405.323\end{array}$

$77.20 \quad 756.000$

NR
37700.000

553.7

$0.7 \quad 1.054$

\begin{tabular}{r|r}
1.70 & 37700.000
\end{tabular}

27614.2

80640.300

$\begin{array}{rr}1.5 & 2.234 \\ 9522.2 & 13000.000\end{array}$

\begin{tabular}{l|l}
1.183 & NR \\
1.245 & NR
\end{tabular}

\begin{tabular}{r|r}
1.351 & 3.610 \\
1.202 & 11.200
\end{tabular}

11.200

1.000145000 .000

\begin{tabular}{r|r}
1.000 & 145000.000 \\
1.000 & 757.000 \\
1.000 & 3370.000
\end{tabular}

$1.000 \quad 3370.000$

NR

2.6

$61.83 \quad 33300.000$

43914
NR

NR $\quad$ NR

NR

\begin{tabular}{r|rrrr}
0.94 & 4.510 & 3.3 & 6.093 \\
0.48 & 2290.000 & 1677.4 & 2752.580 \\
$\leq 100.00$ & NR & NR &
\end{tabular}

$\begin{array}{ll}554.5 & \leq 100.00\end{array}$ NR $\quad$ NR

$\leq 100.00$

2468.4

$\leq 100.00$

1.000
1.000

NR

$\$ 100.00$

$2.450 \quad 619.850 \quad 185.3$

\begin{tabular}{r|r}
1.000 & NR \\
1.000 & 5490,000
\end{tabular}

NR

38.16

$\leq 100,00$

$\begin{array}{ll}N R & N R \\ N R & N R\end{array}$

NR

Sulfate 247370.499

43.78 \%total solids in wash

NR NR

410.000
NR

NR NR

Radionuclides

\begin{tabular}{|c|c|c|c|c|}
\hline \multicolumn{2}{|c|}{ in wash } & \multicolumn{3}{|r|}{ in acid } \\
\hline uCi/g & Cl/tank \% dissolved & $\mathrm{uCi} /$ & Ci/tank & \% dissolve \\
\hline 0.001 & 0.71 & 0.119 & 872 & 61.98 \\
\hline
\end{tabular}

\begin{tabular}{|c|c|c|c|c|}
\hline \multicolumn{2}{|c|}{ in wash } & \multicolumn{3}{|r|}{ in acid } \\
\hline uCi/g & Cl/tank \% dissolved & $\mathrm{uCi} /$ & Ci/tank & \% dissolve \\
\hline 0.001 & 0.71 & 0.119 & 872 & 61.98 \\
\hline
\end{tabular}

171463.624

$30.35 \%$ to

$\begin{array}{lr}0.119 & 872 \\ 0.000 & 0.3\end{array}$

89362

\begin{tabular}{rrr|rr}
0.000 & 0.2 & 42.86 & 0.000 & 8936.2 \\
0.004 & 0.0 & 0.00 & 12.200 & 0.9 \\
& 2.9 & 57.14 & 0.003 & 2.2
\end{tabular}

Sr-90

Tc-99

Am-241

Co-60

Cs-137

NOTES

0.004

0.002

$0.000 \quad 0.0$

0.000

0.0

$0.03 \quad 0.00$

$\begin{array}{ll}0.00 & 0.005 \\ 0.00 & 0.163\end{array}$

1.5

1.5
3.7
119.4
$N R$

1. Nitrate on water leach only, would not account for any nitrate associated with cancrinite

2 No analyses for $\mathrm{Ca}$ B, total $\mathrm{Co}, \mathrm{Cu}, \mathrm{Mg} \mathrm{K}$ total $\mathrm{Sr}$, total $\mathrm{Cs}, \mathrm{Zn}$

3. Reported value of $4.7 \mathrm{e}-5 \mathrm{uCV} / \mathrm{g}$ for I is direct report value for total sample

NR - Not Reported

NA - No Analysis Performed

$<D L$ - Less than Detection Limit 
TY-103 (TBP, BIPO4 1C-F)

\begin{tabular}{|lr|}
\hline Density $(\mathrm{g} / \mathrm{mL})$ & 1.70 \\
Volume (L) & 613170 \\
Mass (kg) & 1042389 \\
Solids (ug/g) & 475000 \\
$\sim \mathrm{pH}$ &
\end{tabular}

\begin{tabular}{lr}
$\begin{array}{l}\text { Solids }(\mathrm{ug} / \mathrm{g}) \\
\sim \mathrm{pH}\end{array}$ & 475000 \\
\hline
\end{tabular}

\begin{tabular}{|c|c|c|c|c|c|c|c|c|c|c|c|c|c|c|}
\hline \multirow[b]{2}{*}{ Component } & \multirow[b]{2}{*}{$\begin{array}{l}\text { Oxide } \\
\text { Factor }\end{array}$} & \multicolumn{3}{|c|}{ - WATER — } & \multicolumn{4}{|c|}{ ACID $\ldots$} & \multicolumn{4}{|c|}{-FUSION } & \multicolumn{2}{|c|}{ ב TOTAL _ _ } \\
\hline & & $\begin{array}{r}\text { ug/g sludge } \\
\text { (element) }\end{array}$ & $\begin{array}{r}\text { kg/tank } \\
\text { (element) }\end{array}$ & $\begin{array}{l}\% \text { element } \\
\text { dissolved }\end{array}$ & $\begin{array}{r}\text { ug/g sludge } \\
\text { (element) }\end{array}$ & $\begin{array}{r}\mathrm{kg} / \operatorname{tank} \\
\text { (element) }\end{array}$ & $\begin{array}{l}\text { ug/g sludge } \\
\text { (oxide) }\end{array}$ & $\begin{array}{c}\text { \% element } \\
\text { dissolved }\end{array}$ & $\begin{array}{l}\text { ug/g sludge } \\
\text { (element) }\end{array}$ & $\begin{array}{r}\mathrm{kg} / \operatorname{tank} \\
\text { (element) }\end{array}$ & $\begin{array}{l}\text { ug/g sludge } \\
\text { (oxide) }\end{array}$ & $\begin{array}{l}\% \text { element } \\
\text { dissolved }\end{array}$ & $\begin{array}{l}\text { ug/g sludge } \\
\text { (element) }\end{array}$ & $\begin{array}{r}\mathrm{kg} / \operatorname{tank} \\
\text { (element) }\end{array}$ \\
\hline Aluminum & 1.889 & 4.240 & 4.4 & 0.05 & 7780.000 & 8109.8 & $14696: 420$ & 94.74 & 428.000 & 446.1 & 808.492 & 5.21 & 8212.240 & 8560.3 \\
\hline Barium & 1.117 & 7.070 & 7.4 & 1.41 & 41.300 & 43.1 & 46.132 & 8.25 & 452.000 & 471.2 & 504.884 & 90.33 & 500.370 & 521.6 \\
\hline Bismuth & 1.115 & 7.040 & 7.3 & 0.03 & 27400.000 & 28561.5 & 30551,000 & 99.95 & 5.510 & 5.7 & 6.144 & 0.02 & 27412.550 & 28574.5 \\
\hline Boron & 3.220 & NR & NR & & NR & NR & & & NR & NR & & & NR & NR \\
\hline Cadmium & 1.142 & 1.610 & 1.7 & 21.10 & 4.870 & 5.1 & 5.562 & 63.83 & $1: 150$ & 1.2 & 1.313 & $15: 07$ & 7.630 & 8.0 \\
\hline Calcium & 1.399 & NR & NR & & NR & NR & & & NR & NR & & & NR & NR \\
\hline Chromium & 1.462 & 406.000 & 423.2 & 40.49 & 588.000 & 612.9 & 859.656 & 58.64 & 8.730 & 9.1 & 12.763 & 0.87 & 1002.730 & 1045.2 \\
\hline Cobalt & 1.407 & NR & NR & & NR & NR & & & NR & NR & & & NR & NR \\
\hline Iron & 1.430 & 156.000 & 162.6 & 0.66 & 22600.000 & 23558.0 & 32318.000 & 94.93 & 1050.000 & 1094.5 & 1501.500 & 4.41 & 23806.000 & 24815.1 \\
\hline Lead: & 1.077 & 5.210 & 5.4 & 1.11 & 441.000 & 459.7 & 474.957 & 94.13 & 22.300 & 23.2 & 24.017 & 4.76 & 468.510 & 488.4 \\
\hline Magnesium & 1.658 & NR & NR & & NR & NR & & & NR & NR & & & NR & NR \\
\hline Manganese & 1.582 & 12.900 & 13.4 & 8.06 & 138.000 & 143.8 & 218.316 & 86.21 & 9.180 & 9.6 & 14.523 & 5.73 & 160.080 & 166.9 \\
\hline Nickel & 1.409 & 63.600 & 66.3 & 2.51 & 1350.000 & 1407.2 & 1902.150 & 53.28 & 1120.000 & 1167.5 & 1578.080 & 44.21 & 2533.600 & 2641.0 \\
\hline Phosphate & 1.000 & 18930.000 & 19732.4 & 30.10 & 43800.000 & 45656.6 & 43800.000 & 69.65 & 158.400 & 165.1 & 158.400 & 0.25 & 62888.400 & 65554.2 \\
\hline Potassium & 1.000 & NR & NR & & NR & NR & & & NR & NR & & & NR & NR \\
\hline Silicon & 2.139 & 72.600 & 75.7 & 0.77 & 514.000 & 535.8 & 1099.446 & 5.46 & 8820.000 & 9193.9 & $18865: 980$ & 93.76 & 9406.600 & 9805.3 \\
\hline Silver & 1.074 & 0.643 & 0.7 & 7.14 & 7.440 & 7.8 & 7.991 & 82.66 & 0.918 & 1.0 & 0.986 & 10.20 & 9.001 & 9.4 \\
\hline Sodium & 1.000 & 73900.000 & 77032.5 & 69.77 & 28600,000 & 29812.3 & 28600.000 & 27.00 & 3420,000 & 3565.0 & 3420.000 & 3.23 & 105920.000 & 110409.8 \\
\hline Strontium & 1.183 & NR & NR & & NR & NR & & & NR & NR & & & NR & NR \\
\hline Zirconium & 1.351 & 2.960 & 3.1 & 5.73 & 4.220 & 4.4 & 5.701 & 8.17 & 44.500 & 46.4 & 60.120 & 86.11 & 51.680 & 53.9 \\
\hline Uranium & 1.202 & 41.400 & 43.2 & 0.25 & 16200.000 & 16886.7 & 19472.400 & 99.71 & 6,060 & 6.3 . & 7.284 & 0.04 & 16247.460 & 16936.2 \\
\hline Nitrate & 1.000 & 149000.000 & 155316.0 & 95.54 & 6950.000 & 7244.6 & 6950.000 & 4.46 & NR & NR & & & 155950.000 & 162560.6 \\
\hline Chloride & 1.000 & 1200.000 & 1250.9 & $\$ 100.00$ & NR & NR & & & NR & NR & & & $=1200,000$ & 1250.9 \\
\hline Fluoride & 1.000 & 860.000 & 896.5 & $\leq 100.00$ & NR & NR & & & NR & NR & & & $>860.000$ & 896.5 \\
\hline Free OH & 1.000 & 86.800 & 90.5 & $\$ 100, \infty$ & NR & NR & & & NR & NR & & & $\geq 86.800$ & 90.5 \\
\hline Carbonate & 1.000 & 4780.000 & 4982.6 & $\leq 100.00$ & NR & NR & & & NR & NR & & & $\geq \overline{4780.000}$ & 4982.6 \\
\hline TOC & 2.450 & 2425.500 & 1032.0 & 66.40 & 501.000 & 522.2 & 1227.450 & 33.60 & NR & NR & & & 1491,000 & 1554.2 \\
\hline Nitrite & 1.000 & 8100.000 & 8443.4 & $\leqslant 100.00$ & NR & NR & & & NR & NR & & & $\geq 8100.000$ & 8443.4 \\
\hline Sulfate & 1.000 & 9260.000 & 9652.5 & 94.56 & \multirow{2}{*}{\multicolumn{4}{|c|}{$\begin{array}{c}182768.180 \\
38.48 \text { \%total solids } \\
\text { in acid }\end{array}$}} & NR: & NR & & & 9793.000 & 10208.1 \\
\hline \multicolumn{2}{|l|}{ Total } & \multicolumn{3}{|c|}{$\begin{array}{c}269323.573 \\
56.70 \text { \%total solids } \\
\text { in wash }\end{array}$} & & & & & \multicolumn{4}{|c|}{$\begin{array}{c}26964.486 \\
5.68 \text { \%total solids } \\
\text { in fusion }\end{array}$} & \multicolumn{2}{|c|}{$\begin{array}{c}100.85 \% \text { mass } \\
\text { balance }\end{array}$} \\
\hline \multicolumn{2}{|l|}{ Radionuclides } & uCi/g & \multicolumn{2}{|c|}{ Ci/tank \% dissolved } & \multicolumn{4}{|r|}{$\%$ dissolved } & uCi/g & $C i / \tan k$ & \multirow{2}{*}{\multicolumn{2}{|c|}{$\begin{array}{r}\% \text { dissolved } \\
0.57\end{array}$}} & uCi/g & Cij/tank \\
\hline Pu-239,240 & & 0.000 & 0.2 & 0.10 & 0.175 & 182.4 & & 99.34 & 0.001 & 1.0 & & & 0.176 & 183.6 \\
\hline \multicolumn{2}{|l|}{$c-14$} & 0.001 & 1.0 & 71.43 & 0.0004 & 0.4 & & 28.57 & NR & NR & & & 0.001 & 1.5 \\
\hline \multirow{2}{*}{\multicolumn{2}{|c|}{$\begin{array}{l}\text { St-90 } \\
\text { TC-99 }\end{array}$}} & 0.009 & 9.4 & 0.01 & 105.000 & 109450.8 & & 99.97 & 0.024 & 25.0 & & 0.02 & 105.033 & 109485.2 \\
\hline & & 0.011 & 11.5 & 68.75 & 0.005 & 5.2 & & 31.25 & 0.000 & 0.0 & & 0.00 & 0.016 & 16.7 \\
\hline \multicolumn{2}{|l|}{ Am-241. } & 0.001 & 10 & 3.70 & 0.025 & 26.1 & & 92.59 & 0.001 & 1.0 & & 3.70 & 0.027 & 28.1 \\
\hline \multicolumn{2}{|l|}{ Co-60 } & 0.001 & 1.0 & 20.00 & 0.003 & 3.1 & & 60.00 & 0.001 & 1.0 & & 20.00 & 0.005 & 5.2 \\
\hline
\end{tabular}

NOTES:

1. No analyses for $\mathrm{Ca}, \mathrm{B}$, total $\mathrm{Co}, \mathrm{Cu}, \mathrm{Mg}, \mathrm{K}$, total $\mathrm{Sr}$, total $\mathrm{Cs}, \mathrm{Zn}$

2. Reported value of $<2.7 \mathrm{e}-3 \mathrm{uCi} / \mathrm{g}$ for $I$ is direct report value for total sample

NR - Not Reported

NA - No Analysis Performed

$<D L$ - Less than Detection Limit 


\begin{tabular}{|c|c|c|c|c|c|c|c|c|c|c|c|c|c|c|}
\hline $\begin{array}{l}\text { Density (g/mL) } \\
\text { Volume (L) } \\
\text { Mass (kg) } \\
\text { Solids (ug/g) } \\
\sim \text { pH } \\
\end{array}$ & $\begin{array}{r}1.69 \\
162755 \\
275056 \\
430250 \\
\text { NR } \\
\end{array}$ & (drainable liqu & $\mathrm{pH}$ 12.1) & & & & & & & & & 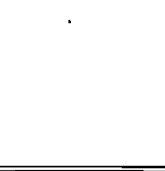 & & \\
\hline Component & $\begin{array}{l}\text { Oxide } \\
\text { Factor }\end{array}$ & $\begin{array}{r}\text { ug/g sludge } \\
\text { (element) }\end{array}$ & $\begin{array}{r}\text { WATER } \\
\text { kg/tank } \\
\text { (element) }\end{array}$ & $\begin{array}{l}\text { \% element } \\
\text { dissolved }\end{array}$ & $\begin{array}{r}\text { ug/g sludge } \\
\text { (element) }\end{array}$ & \multicolumn{2}{|c|}{$\begin{array}{c}\text { kg/tank ug/g sludge } \\
\text { (element) }\end{array}$} & $\begin{array}{l}\text { \% element } \\
\text { dissolved }\end{array}$ & $\begin{array}{l}\text { ug/g sludge } \\
\text { (element) }\end{array}$ & \multicolumn{2}{|c|}{$\begin{array}{l}\text { kg/tank ug/g sludge } \\
\text { (element) }\end{array}$} & $\begin{array}{l}\text { \% element } \\
\text { dissolved }\end{array}$ & $\begin{array}{l}\text { ug/g sludge } \\
\text { (element) }\end{array}$ & $\begin{array}{r}\overline{\mathrm{AL}} \overline{\mathrm{kg} / \operatorname{tank}} \\
\text { (element) }\end{array}$ \\
\hline Aluminum & 1.889 & 414.500 & 114.0 & 4.56 & 6957.500 & 1913.7 & 13142.718 & 76.54 & 1718.500 & 472.7 & 3246.247 & 18.90 & 9090.500 & 2500.4 \\
\hline Barium & 1.117 & 0.948 & 0.3 & 0.21 & 52.950 & 14.6 & 59.145 & 11.96 & 389.000 & 107.0 & 434.513 & 87.83 & 442.898 & 121.8 \\
\hline Bismuth & 1.115 & 10.055 & 28 & 0.06 & 17650.000 & 48547 & 19679.750 & 9942 & 92.758 & 25.5 & 103.425 & 0.52 & .17752 .813 & 4883.0 \\
\hline Boron & 3.220 & NR & NR & & NR & NR & & & NR & NR & & & NR & NR \\
\hline Cadmium & 1.142 & 3.140 & 0.9 & 24.48 & 8.398 & 2.3 & 9.590 & 65.48 & 1.288 & 0.4 & 1.470 & 10.04 & 12.825 & 3.5 \\
\hline Calcium & 1.399 & NR & NR & & NR & NR & & & NR & NR & & & NR & NR \\
\hline Chromium & 1.462 & 1794.000 & 493.5 & 77.63 & 509.250 & 140.1 & 744.524 & 22,04 & 7.813 & 2.1 & 11.422 & 0.34 & 2311.063 & 635.7 \\
\hline Cobalt & 1.407 & NR & NR & & NR & NR & & & NR & NR & & & NR & NR \\
\hline Copper & 1.252 & NR & NR & & NR & NR & & & NR & NR & & & NR & NR \\
\hline Iron & 1.430 & 85.775 & 23.6 & 0.26 & 31325.000 & 8616.1 & 44794.750 & 96.18 & 1159.750 & 319.0 & 1658.443 & 3.56 & 32570.525 & 8958.7 \\
\hline Lead & 1.077 & 10.263 & 2.8 & 177 & 558,500 & 153.1 & 599.351 & 96.02 & 12.818 & 3.5 & 13.804 & 2.21 & 579.580 & 159.4 \\
\hline Magnesium & 1.658 & NR & NR & & NR & NR & & & NR & NR & & & NR & NR \\
\hline Manganese & 1582 & 15.018 & 4.1 & 0.72 & 2060.000 & 566.6 & 3258.920 & 98.63 & 13.645 & 3.8 & 21.586 & 0.65 & 2088.663 & 574.5 \\
\hline Nickel & 1.409 & 16.650 & 4.6 & 1.07 & 1440.000 & 396.1 & 2028.960 & 92.69 & 96.985 & 26.7 & 136.652 & 6.24 & 1553.635 & 427.3 \\
\hline Phosphate & 1.000 & 53332.500 & 14669.4 & 67.87 & 24825.000 & 6828.3 & 24825.000 & 31.59 & 422.775 & 116.3 & 422.775 & 0.54 & 78580.275 & 21614.0 \\
\hline Potassium & 1.000 & NR & NR & & NR & NR & & & NR & NR & & & NR & NR \\
\hline Silicon & 2.139 & 162.650 & 44.7 & 2.01 & 229.750 & 63.2 & 491.435 & 2.84 & 7700.000 & 2117.9 & 16470.300 & 95.15 & 8092.400 & 2225.9 \\
\hline Silver & 1.074 & 2.785 & 0.8 & 22.48 & 8.818 & 2.4 & 9.470 & 71.17 & 0.788 & 0.2 & 0.846 & 6.36 & 12.390 & 3.4 \\
\hline Sodium & 1.000 & 86150,000 & 23696.1 & 76.15 & 24025,000 & 6608.2 & 24025.000 & 21.24 & 2962,500 & 814.9 & 2962.500 & 2.62 & 113137.500 & 31119.1 \\
\hline Strontium & 1.183 & NR & NR & & NR & NR & & & NR & NR & & & NR & NR \\
\hline Zinc & 1.245 & NR & NR & & NR & $\mathrm{NR}$ & & & NR & NR & & & NR & NR \\
\hline Zirconium & 1.351 & 4.710 & 1.3 & 2.59 & 5.300 & 1.5 & 7.160 & 2.92 & 171.500 & 47.2 & 231.697 & 94.49 & 181.510 & 49.9 \\
\hline Uranlum & 1202 & 37.950 & 10.4 & 0,19 & 19300.000 & 5308.6 & 23198.600 & 99.16 & 124.708 & 34.3 & 149.899 & 0.64 & 19462.658 & 5353.3 \\
\hline Nitrate & 1.000 & 44675.000 & 12288.1 & 95.87 & 1922.500 & 528.8 & 1922.500 & 4.13 & NR & NR & & & 46597.500 & 12816.9 \\
\hline Chloride & 1.000 & 598.000 & 164.5 & $\$ 100.00$ & NR & NR & & & NR & NR & & & $\geq 598.000$ & 164.5 \\
\hline Fluoride & 1.000 & 5352.500 & 1472.2 & $\leq 100.00$ & NR & NR & & & NR & NR & & & $\geq 5352.500$ & 1472.2 \\
\hline Free OH & 1.000 & 1289,500 & 3547 & $\leq 100.00$ & NR & NR & & & NR & NR & & & $\geq 1289.500$ & 354.7 \\
\hline Carbonate & 1.000 & 18250.000 & 5019.8 & $\leq 100.00$ & NR & NR & & & NR & NR & & & $\geq 18250.000$ & 5019.8 \\
\hline TOC & 2.450 & 4012.488 & 450.5 & 84.54 & 299.500 & 82.4 & 733.775 & 15.46 & NR & NR & & & 1937.250 & 532.9 \\
\hline Nitrite & 1.000 & 12217.500 & 3360.5 & $\leq 100.00$ & NR & NR & & & NR & NR & & & $\geq 12217.500$ & 3360.5 \\
\hline Sulfate & 1.000 & & 1113.3 & 7746 & \multirow[t]{2}{*}{1177500} & 323.9 & O & 22.54 & NR & NR & & & 5225.000 & 1437.2 \\
\hline \multicolumn{2}{|l|}{ Total } & \multicolumn{3}{|c|}{$\begin{array}{c}232483.431 \\
54.03 \% \text { \%otal solids } \\
\text { in wash }\end{array}$} & & & $\begin{array}{r}160708.147 \\
37.35 \\
\end{array}$ & $\begin{array}{l}\text { \%total solids } \\
\text { in acid }\end{array}$ & & & $\begin{array}{r}25865.578 \\
6.01 \\
\end{array}$ & $\begin{array}{l}\text { \%total solids } \\
\text { in fusion }\end{array}$ & \multicolumn{2}{|c|}{$\begin{array}{c}97.40 \% \text { mass } \\
\text { balance }\end{array}$} \\
\hline \multicolumn{2}{|l|}{ Radionuclides } & uCi/g & Ci/tank & 6 dissolved & uCi/g & Ci/tank & & \% dissolved & uCi/g & Ci/tank & & \% dissolved & $\mathbf{u C i} / \mathrm{g}$ & Ci/tank \\
\hline \multicolumn{2}{|l|}{ Pu-23: } & 0.001 & 0.2 & 0.47 & 0.173 & 476 & & 95.66 & 0.007 & 1.9 & & 3.87 & 0.181 & 49.7 \\
\hline \multicolumn{2}{|l|}{$C-14$} & 0.002 & 0.6 & 64.52 & 0.001 & 0.3 & & 35.48 & 0.000 & 0.0 & & 0.00 & 0.003 & 0.9 \\
\hline St-90 & & 0.039 & 10.8 & 0.03 & 131250 & 361011 & & 99.69 & 0.366 & 100.5 & & 0.28 & 131.655 & 36212.4 \\
\hline Tc-99 & & 0.024 & 6.6 & 73.28 & 0.007 & 1.9 & & 20.61 & 0.002 & 0.6 & & 6.11 & 0.033 & \\
\hline Am-241 & & 0.002 & 0.6 & 5.97 & 0.029 & 78 & & 85.07 & 0.003 & 0.8 & & 8.96 & 0.034 & 9.2 \\
\hline Co-60 & & 0.003 & 0.8 & 12.17 & 0.018 & 5.0 & & 80.75 & 0.002 & 0.4 & & 7.08 & 0.023 & 6.2 \\
\hline Cs-137 & & 16,455 & 4526,0 & 36.26 & 10.195 & 2804.2 & & 22.47 & 18.727 & 5151.0 & & 41.27 & 45.377 & 12481.2 \\
\hline $1-129$ & & 0.0000335 & 0.01 & $\leq 100.00$ & NR & NR & & & NR & NR & & & $\geq 0.0000335$ & 0.01 \\
\hline
\end{tabular}

NOTES

1. No analyses for $\mathrm{Ca}, \mathrm{B}$, total $\mathrm{Co}, \mathrm{Cu}, \mathrm{Mg}, \mathrm{K}$, total $\mathrm{Sr}$, total $\mathrm{Cs}, \mathrm{Zn}$

2. Reported value of $3.35 e-5 \mathrm{uCi} / \mathrm{g}$ for I is direct report value for total sample

NR - Not Reported

NA - No Analysis Performed

$<D L$ - Less than Detection Limit 


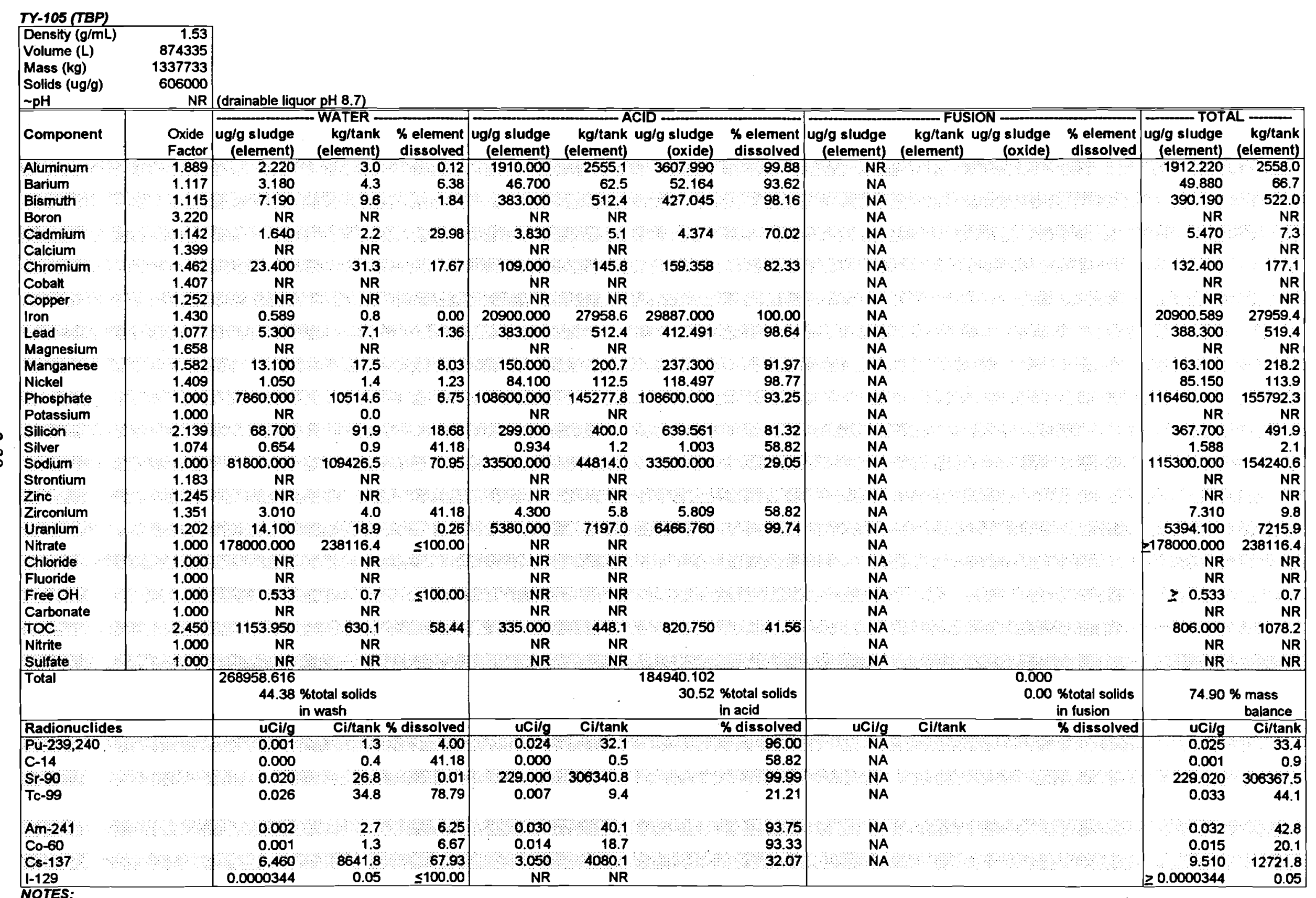

1. No analyses for $\mathrm{Ca}, \mathrm{B}$, total $\mathrm{Co}, \mathrm{Cu}, \mathrm{Mg}, \mathrm{K}$, total $\mathrm{Sr}$, total $\mathrm{Cs}, \mathrm{Zn}$

2. Reported value of $4.7 \mathrm{e}-5 \mathrm{uCi} / \mathrm{g}$ for $\mathrm{I}$ is direct report value for total sample
NR - Not Reported

NA - No Analysis Performed

$<D L$ - Less than Detection Limit 
TY-106 (TBP, $27 t$ dlatomaceous earth)

Density $(\mathrm{g} / \mathrm{mL}) \quad 1.37$

Volume (L) $\quad 64345$

Mass (kg) $\quad 88153$

$\begin{array}{lr}\text { Solids }(\mathrm{ug} / \mathrm{g}) & 638500 \\ \sim \mathrm{pH} & \mathrm{NR} \text { (drainable liquor } \mathrm{pH} \sim 9 \text { ) }\end{array}$

\begin{tabular}{|c|c|c|c|c|c|c|c|c|c|c|c|c|c|c|}
\hline \multirow{2}{*}{ Component } & \multirow[b]{2}{*}{$\begin{array}{l}\text { Oxide } \\
\text { Factor }\end{array}$} & \multicolumn{3}{|c|}{ WATER ___ W_ } & \multirow[b]{2}{*}{$\begin{array}{l}\text { ug/g sludge } \\
\text { (element) }\end{array}$} & \multirow{2}{*}{\multicolumn{2}{|c|}{ kg/tank ug/g sludge }} & \multirow[b]{2}{*}{$\begin{array}{l}\% \text { element } \\
\text { dissolved }\end{array}$} & \multirow{2}{*}{$\begin{array}{l}\text { ug/g s ludge } \\
\text { (element) }\end{array}$} & \multirow{2}{*}{\multicolumn{2}{|c|}{$\begin{array}{r}\text { kg/tank ug/g sludge } \\
\text { (element) }\end{array}$}} & \multirow[b]{2}{*}{$\begin{array}{l}\text { \% element } \\
\text { dissolved }\end{array}$} & \multicolumn{2}{|c|}{ TOTAL } \\
\hline & & $\begin{array}{l}\text { ug/g sludge } \\
\text { (element) }\end{array}$ & $\begin{array}{r}\mathrm{kg} / \mathrm{tank} \\
\text { (element) }\end{array}$ & $\begin{array}{l}\text { \% element } \\
\text { dissolved }\end{array}$ & & & & & & & & & $\begin{array}{l}\text { ug/g sludge } \\
\text { (element) }\end{array}$ & $\begin{array}{r}\text { kg/tank } \\
\text { (element) }\end{array}$ \\
\hline Juminur & 1.889 & 3.075 & 0.3 & 0.04 & 1241.000 & 109.4 & 2344.249 & 18.12 & 5605.000 & 494.1 & 10587.845 & 81.84 & 6849.075 & 603.8 \\
\hline Sarium & 1.117 & 7.879 & 0.7 & 0.60 & 570.500 & 50.3 & 637.249 & 43.55 & 731.500 & 64.5 & 817.086 & 55.84 & 1309.879 & 115.5 \\
\hline Bismuth & 1.115 & 9.840 & 0.9 & 188 & 425.000 & 37.5 & 473.875 & 81.12 & 89.050 & 7.8 & 99.291 & 1700 & 523.890 & 46.2 \\
\hline Boron & 3.220 & NR & NR & & NR & NR & & & NR & NR & & & NR & NR \\
\hline Sadmium & 1.142 & 2.260 & 0.2 & 7.94 & 5.865 & 0.5 & 6.698 & 20.60 & 20.350 & 1.8 & 23.240 & 71.47 & 28.475 & 2.5 \\
\hline alcium & 1.399 & NR & NR & & NR & NR & & & NR & NR & & & NR & NR \\
\hline Chromium & 1.462 & 1.505 & 0.1 & 1.06 & 112.450 & 9.9 & 164.402 & 79.05 & 28.300 & 2.5 & 41.375 & 19.89 & 142.255 & 12.5 \\
\hline Cobalt & 1.407 & NR & NR & & NR & NR & & & NR & NR & & & NR & NR \\
\hline pper: & 1.252 & NR & NR & & NR & NR & & & NR & NR & & & NR & NR \\
\hline on & 1.430 & 4.363 & 0.4 & 0.01 & 24200.000 & 2133.3 & 34606.000 & 52.84 & 21590.000 & 1903.2 & 30873.700 & 47.15 & 45794.363 & 4036.9 \\
\hline$e a$ & 1.077 & 7.330 & 0.6 & 191 & 280,000 & 24.7 & 301 & 73.02 & $96: 150$ & 8.5 & 10 & 25.07 & 383.480 & 33.8 \\
\hline sium & 1.658 & NR & NR & & NR & NR & & & NR & NR & & & NR & NR \\
\hline Manganese & 1.582 & 18.100 & 16 & 3.27 & 181.000 & 16.0 & 286.342 & 32.67 & 355.000 & 31,3 & 561.610 & 64.07 & 554.100 & 48.8 \\
\hline ick & 1.409 & 30 & 0.1 & 2.12 & .050 & 4.2 & 67 & 70.40 & 750 & 1.7 & & 27.47 & 68.250 & 6.0 \\
\hline Ph & 1.000 & 12555.000 & 1106.8 & 21.71 & 43800,000 & 3861.1 & 43800.000 & 75.72 & 1488.000 & 131.2 & 1488 & 2.57 & 57843.000 & 5099.0 \\
\hline Potas & 1.000 & NR & NR & & NR & NR & & & NR & NR & & & NR & NR \\
\hline Silicon & 2139 & 55900 & 49 & 0.08 & 632.000 & 55.7 & 1351. & 0.71 & 88250.000 & .7779 .5 & 188766 & 9.23 & 88937.900 & 7840.1 \\
\hline & 1.074 & & 0.1 & 3.27 & 523 & 1.9 & 5 & 77.73 & 5.260 & 0.5 & & 9.00 & 27.689 & 2.4 \\
\hline So & 1.000 & 79600.000 & 7017.0 & 75.32 & 17950.000 & 1582.3 & 17950000 & 16.99 & 8130,000 & 716.7 & 8130.000 & 7.69 & 105680.000 & 9316.0 \\
\hline um & 1.183 & NR & NR & & NR & NR & & & NR & NR & & & NR & NR \\
\hline & 1245 & NR & NR & & NR & NR & & & NR & NR & & & NR & NR \\
\hline Zir & 1.351 & 4.160 & 0.4 & 0.64 & 9.625 & 0.8 & 13.003 & 1.49 & 633.500 & 55.8 & 359 & 97.87 & 647.285 & 57.1 \\
\hline & 1202 & 28 & 0.2 & 0.03 & 8125 & 7162 & 976 & 97.31 & 000 & 19.6 & 266.844 & 266 & 9.801 & 736.1 \\
\hline $\mathrm{Nit}$ & 1.000 & 166500.000 & 14677.4 & 97.28 & 4662.000 & 411.0 & 4662.000 & 2.72 & NR & NR & & & 171162.000 & 15088.4 \\
\hline & 1000 & 1232.100 & 108.6 & $\leq 100.00$ & NR & NR & & & NR & NR & & & $\geq 1232.100$ & 108.6 \\
\hline Flu & 1.000 & 699.300 & 61.6 & 0.00 & NR & NR & & & NR & NR & & & 99.300 & 61.6 \\
\hline Fre & 1.000 & 19 & 0.1 & 000 & NR & NR & & & NR & NR & & & $\geq 1.049$ & 0.1 \\
\hline onate & 1.000 & 1015.650 & 89.5 & $\leq 100.00$ & NR & NR & & & NR & NR & & & $>1015.650$ & 89.5 \\
\hline TO & 2,450 & 3797.500 & 136.6 & 6794 & 731.500 & 64.5 & 1792.175 & 32.06 & NR & NR & & & 2281.500 & 201.1 \\
\hline & 1.000 & 5661.000 & 499.0 & 100.00 & NR & NR & & & NR & NR & & & 1.000 & 499.0 \\
\hline su & 1.000 & 11988.000 & 1056.8 & & $\infty 00$ & 1719 & 1950.000 & 13,99 & NF & NR & & & .000 & 1228.7 \\
\hline \multicolumn{2}{|l|}{ Total } & \multicolumn{3}{|c|}{$\begin{array}{c}283169.166 \\
44.35 \% \text { \%total solids } \\
\text { in wash }\end{array}$} & & \multicolumn{3}{|c|}{$\begin{array}{c}120196.468 \\
18.82 \text { \%total solids } \\
\text { in acid }\end{array}$} & \multicolumn{4}{|c|}{$\begin{array}{c}242647.220 \\
38.00 \text { \%total solids } \\
\text { in fusion }\end{array}$} & \multicolumn{2}{|c|}{$\begin{array}{r}101.18 \% \text { mass } \\
\text { balance }\end{array}$} \\
\hline \multicolumn{2}{|l|}{ Radionuclides } & $\mathrm{uCi} / \mathrm{g}$ & Ci/tank 9 & solved & $\mathrm{uCi} / \mathrm{g}$ & Ci/tank & & \% dissolved & $\mathrm{uCi} / \mathrm{g}$ & Ci/tank & & \% dissolved & $\mathrm{uCi} / \mathrm{g}$ & Ci/tank \\
\hline \multicolumn{2}{|l|}{$\mathrm{Pu}-239,240$} & 0.001 & 01 & 1.72 & 0.033 & 2.9 & & 81.08 & 0.007 & 0. & & 17.20 & 0.041 & 3.6 \\
\hline \multicolumn{2}{|l|}{ C.14 } & & 0.0 & 40.00 & & 0.1 & & & & 0. & & 0.00 & 0.001 & 0.1 \\
\hline \multicolumn{2}{|l|}{ Sr-90 } & 0.058 & 51 & 0.04 & 133.500 & 11768.4 & & 98.34 & 2195 & 193.5 & & 1.62 & 135.752 & 11966.9 \\
\hline \multicolumn{2}{|l|}{ Tc-99 } & 0.105 & 9.3 & 86.42 & 0.017 & 1.5 & & 13.58 & 0.000 & 0.0 & & 0.00 & 0.122 & 10.7 \\
\hline Am-241 & & 0.006 & 0.5 & 12.37 & & 22 & & & & 1. & & 11 & 0.0 & \\
\hline $00-60$ & & 0.001 & 0.1 & 3.69 & 0.007 & 0.6 & & & 0.024 & 2.1 & & 44 & 0.031 & 2.7 \\
\hline Cs- 137 & & 4.930 & 434,6 & 2077 & 9705 & 8555 & & 40.89 & 9100 & 802.2 & & 38.34 & 23.735 & 2092.3 \\
\hline & & 0.001 & & 0.00 & & & & & & & & & 001 & \\
\hline
\end{tabular}

i-129:

0.001

$0.1 \leq 100.00$

NR NR

NR NR

$\geq 0.001$

0.1

1. No analyses for $\mathrm{Ca}, \mathrm{B}$, total $\mathrm{Co}, \mathrm{Cu}, \mathrm{Mg}, \mathrm{K}$, total $\mathrm{Sr}$, total $\mathrm{Cs}, \mathrm{Zn}$

NR - Not Reported

NA - No Analysis Performed

$<D L$ - Less than Detection Limit 


\begin{tabular}{ll}
$\sim \mathrm{pH}$ & $12.6-13$ \\
\hline
\end{tabular}

\section{ugra sludge}

WATER

kg/tank \% element ug/g sludge $\mathrm{ACID}$

ludge \% element ug/g sludge kg/OUN

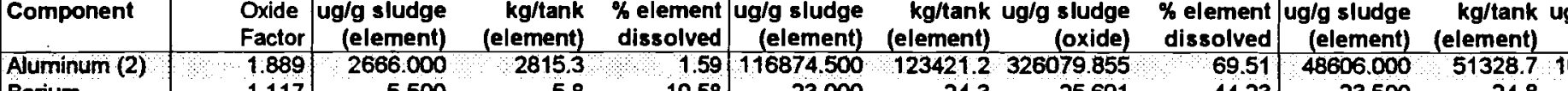
Barium Bismuth Boron

Cadmium

Calcium

Chromium

Cobalt

Copper

Iron

Lead

Magnesium

Mangan
Nickel

Phosphate (3)

Potassium

Silicon

Sodium

Strontium

Zinc:

Zirconium

Uranium

Nitrate

Chloride

Free $\mathrm{OH}$ (4)

Carbonate

TOC

Nitrite

\begin{tabular}{ll} 
Nitrite & 1.000 \\
sulfate & 1.000 \\
\hline Total &
\end{tabular}

$\begin{array}{lll}23.000 & 24.3 & 25.691\end{array}$

$<\mathrm{DL}$

$5.8 \quad 10.58$

9421.500

$9949.2 \quad 10504.973$

1.115
3.220
1.142

285.500

301.5

109.000

232.500

115.1

$100.00 \quad 0.000$

16.000
206.500

$\begin{array}{rr}0.0 & 0.000\end{array}$

123.6

\begin{tabular}{r|r}
3.83 & 206.500 \\
59.39 & 159.000
\end{tabular}

$\begin{array}{rr}16.9 & 18.272 \\ 218.1 & 288.894\end{array}$

1.462

117.000
$<D L$

100.00

NR
184.500

$\begin{array}{ll}167.9 & 232.458\end{array}$

32.000

338

\begin{tabular}{r|r}
184.500 \\
0.32 & 7603.000
\end{tabular}

$57.000 \quad 60.2$

$1.077 \quad 57.000$

334.8

$\angle D L$

1.582
1.409

$1.409 \quad<D L$

\begin{tabular}{r|r}
1.000 & 43880.000 \\
1.000 & $<600$
\end{tabular}

\begin{tabular}{r|r}
1.000 & $<600$ \\
2.139 & 822.500
\end{tabular}

46337 :

11.60
23.31

$194.8 \quad 230.994$

$8028.9 \quad 10872.290$

$\begin{array}{llll}34.500 & 458.8 & 467.957\end{array}$

$\begin{array}{rrr}2357.500 & 0.0 & 0.000\end{array}$

$\begin{array}{rrr}2357.500 & 2489.6 & 3729.565 \\ 93.500 & 98.7 & 131.742\end{array}$

$0.000 \quad 0.0 \quad 0.000$

$<633$
868.6

100.00

$\begin{array}{llll}1210.000 & 1277.8 & 1210.000\end{array}$

$<D L$

\begin{tabular}{r|r}
1.000 & 65779.500 \\
1.183 & 3.500
\end{tabular}

\begin{tabular}{rr|r} 
& 7.53 & NR \\
694641 & 60.61 & 67.500
\end{tabular}

NR

72.495

$\begin{array}{rrr}11697.000 & 12352.2 & 11697.000 \\ 294.000 & 310.5 & 347.802\end{array}$

\begin{tabular}{r|r}
0.87 & 294.000
\end{tabular}

$\begin{array}{ll}119.9 & 347.802 \\ 149.4 & 141.308\end{array}$

3.7
26.9

$13.97 \quad 113.500$

141.500

$\begin{array}{ll}119.9 & 141.308 \\ 149.4 & 191.167\end{array}$

1.245
1.351 $\quad \begin{array}{r}25.500 \\ 1.202\end{array}$

$<3000$

$<3170$

$\begin{array}{lll}1.000 & 31750.000 & 33528.5\end{array}$

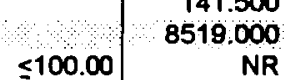

$\begin{array}{rr}149.4 & 191.167 \\ 8996.2 & 10239.838\end{array}$

$1.000 \quad 975.000$

1.000
1.000

1029.6

$\$ 100.00$

$\leq 100.00$

$5280.1 \quad \leq 100.00$

$1000 \quad 16025.000 \quad 16922$

$\leq 100.00$

$\leq 100.00$

450 3317300

$1.000 \quad 6620.000$

$\leq 100.00$

1277.8

93.80

186657.300

NR $\quad$\begin{tabular}{rr}
64.46 \\
\hline
\end{tabular}$\quad 100.00$

$\begin{array}{r}23.500 \\ \hline 6.23 \quad 4.500\end{array}$

6754

23.500
4528.500

(oxide)

933.200

\begin{tabular}{r|r}
67.54 & 4528.500 \\
0.00 & 0.000
\end{tabular}

7.26
2530.500

5049.278

$\begin{array}{rr}0.0 & 0.000\end{array}$

$\begin{array}{rr}0.0 & 0.000 \\ 2672.2 & 3540.170 \\ 0.0 & 0.000\end{array}$

0.000
$<250$
103.500

$\begin{array}{rrrrr}64.06 & 103.500 & 109.3 & 129.582\end{array}$

$\begin{array}{llll}75.44 & 2443.500 & 2580.4 & 3494.205\end{array}$

$\begin{array}{lrrrr}88.40 & 0.000 & 0.0 & 0.000\end{array}$

$0.00 \quad 1043.000$

$100.00 \quad 0.000$

\begin{tabular}{r|r}
2.62 & 3473.000 \\
0.00 & NR
\end{tabular}

100.00

$100 \quad \begin{array}{r}\text { NA } \\ 10097.500\end{array}$

\begin{tabular}{l|r}
10.78 & 31059.500 \\
73.13
\end{tabular}

$73.13 \quad 104.500$

$6210 \quad 43.500$

64.46

43.500

78.000
0.000

1101.4

0.0
3667.5

NR

1729.294

1729.294
0.000

10663.1 21598.553

$<130$

$32799.3 \quad 31059.500$

$110.4 \quad 123.624$

$45 . \quad 54.158$

$82 . \quad 105.378$

$0.0 \quad 0.000$

element ug/g sludge

(element) (element)

NR NR

$\begin{array}{ll}\text { NR } & \text { NR } \\ \text { NR } & \text { NR }\end{array}$

NR $\quad$ NR

NR NR NR

\begin{tabular}{r|r}
25.17 \%total solids \\
in wash
\end{tabular}

0.000

N.0.

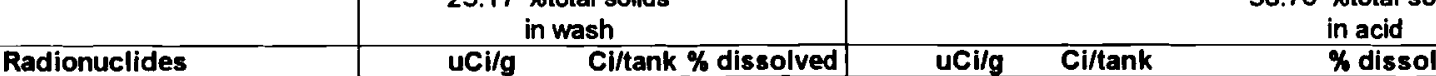

Pu-239,240

C-14

Sr-90

TC-99

Am-241

Co-60

Cs-137

Cs- 137
I-129

0.001

$1 / \operatorname{tank}$

\begin{tabular}{r|r}
0.65 & $\mathrm{NA}$ \\
100.00 & $\mathrm{NA}$
\end{tabular}

NA

NA

0.003

243.9

0.09
68.20

$-0.002 \curvearrowright 21 \% 0.00$

NR N NR

$<0.005$

$<5.3$

22.55

NA

NA

NA

a

1. Core Composites 7 and 14 had good segement recovery and were chosen to represent $U-110$

2. Acid soluble oxide factor 2.79 (85\% Al(OH)3,15\% AlOOH); fusion soluble oxide factor 2.22 (AIOOH)

3. Na7F(PO4)2 has been I.D.'d (XRD); this sait dissolves in water
4. Free $\mathrm{OH}$ estimated based on concentration of $\mathrm{Al}$ in water wash 


\subsection{Data References}

Tank I.D.

A-102 Weiss, R. L. and K. E. Schull. 1988. Data Transmittal Package for 241-A-102 Waste Tank Characterization, SD-RE-TI-201, Rev. 0.

A-103 Weiss, R. L. and K. E. Schull. 1988. Data Transmittal Package for 241-A-103 Waste Tank Characterization, SD-RE-TI-198, Rev. 0.

A-106 Weiss, R. L. and K. E. Schull. 1988. Data Transmittal Package for 241-A-106 Waste Tank Characterization, SD-RE-TI-200, Rev. 0.

B-110 Jones, T. E., S. G. McKinley, J. M. Tingey, T. M. Longaker, J. A. Gibson. 1990. SST Waste Characterization Project: (241-B-110) Cores 1 - 4 Data Reports.

B-111 Giamberardini, K. K. 1993. PNL 325 Laboratory Single-Shell Tank Waste Characterization, Tank B-111, Cores 29 and 30, WHC-SDWM-DP-041.

B-201 1993. PNL 325 Laboratory Single-Shell Tank Waste Characterization Tank 241-B-201, Cores 26 and 27 Data Package, WHC-SD-WM-DP037, Rev. 0.

BX-104 Weiss, R. L. and K. E. Schull. 1988. Data Transmittal Package for 241-BX-104 Waste Tank Characterization, SD-RE-TI-206, Rev. 0.

BX-105 Weiss, R. L. and K. E. Schull. 1988. Data Transmittal Package for 241-BX-105 Waste Tank Characterization, SD-RE-TI-202, Rev. 0.

BX-107 Kristofski, J. G. 1993. Single-Shell Tank Waste Characterization 241-BX-107 Cores 40 and 41, "Sample Data Summary," WHC-SDWM-DP-028, Addendum 2, Rev. 0.

C-103 Weiss, R. L. and K. E. Schull. 1988. Data Transmittal Package for 241-C-103 Waste Tank Characterization, SD-RE-TI-203, Rev. 0.

C-104 Weiss, R. L. and K. E. Schull. 1988. Data Transmittal Package for 241-C-104 Waste Tank Characterization, SD-RE-TI-199, Rev. 0.

C-105 Weiss, R. L. and K. E. Schull. 1988. Data Transmittal Package for 241-C-105 Waste Tank Characterization, SD-RE-TI-204, Rev. 0. 
C-106 Weiss, R. L. and K. E. Schull. 1988. Data Transmittal Package for 241-C-106 Waste Tank Characterization, SD-RE-TI-205, Rev. 0.

C-109 Simpson, B. C., G. L. Borsheim, L. Jensen. 1993. Tank

Characterization Report: Tank 241-C-109, WHC-EP-0668.

C-110 1993. Westinghouse 222-S Analytical Laboratory Single-Shell Tank Waste Characterization Tank C-110 Cores 37, 38 and 39, WHC-SDWM-DP-027, Addendum 1, Rev. 0.

C-112 Simpson, B. C., G. L. Borsheim, L. Jensen. 1993. Tank

Characterization Data Report: Tank 241-C-112, WHC-EP-0640.

S-104 Kocher, K. L. 1993. Single-Shell Tank Waste Characterization Tank 241-S-104 Data Package, "222-S Laboratory Single-Shell Tank Waste Characterization, Tank S-104 Cores 42, 43 and 44 Validation Summary," WHC-SD-WM-DP-031, Addendum 2 Rev. 0.

T-102 1993. PNL 325 Laboratory Single-Shell Tank Waste Characterization Tank T-102, Cores 55 and 56 Validation Summary, WHC-SD-WMDP-052, Addendum 1, Rev. 0.

T-104 1993. Westinghouse 222-S Laboratory Single-Shell Tank Waste Characterization, Tank 241-T-104 Cores 45 and 46 Validation Summary, WHC-SD-WM-DP-032, Addendum 1, Rev. 0.

T-107 1993. Westinghouse 222-S Laboratory Single-Shell Tank Waste Characterization, Tank T-107 Cores 50, 51, and 52 Validation Summary, WHC-SD-WM-042, Addendum 1A, Rev. 1.

T-111 1993. Westinghouse 222-S Laboratory Single-Shell Tank Waste Characterization, Tank 241-T-111 Cores 31 and 33 Validation Report, WHC-SD-WM-DP-024, Addendum 1, Rev. 0.

TY-101 Weiss, R. L. and B. M. Mauss. 1987. Data Transmittal Package for 241-TY-101 Waste Tank Characterization, SD-RE-TI-185, Rev. 0.

TY-103 Weiss, R. L. and B. M. Mauss. 1987. Data Transmittal Package for 241-TY-103 Waste Tank Characterization, SD-RE-TI-184, Rev. 0.

TY-104 Weiss, R. L. and B. M. Mauss. 1987. Data Transmittal Package for 241-TY-104 Waste Tank Characterization, SD-RE-TI-182, Rev. 0. 
TY-105 Weiss, R. L. and B. M. Mauss. 1987. Data Transmittal Package for 241-TY-105 Waste Tank Characterization, SD-RE-TI-186, Rev. 0.

TY-106 Weiss, R. L. and B. M. Mauss. 1987. Data Transmittal Package for 241-TY-106 Waste Tank Characterization, SD-RE-TI-181, Rev. 0.

U-110 Brown, T. M. and L. Jensen. 1993. Tank Characterization Report for Single-Shell Tank 241-U-110, WHC-EP-0643, Rev. 1. 


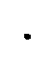




\subsection{Sludge Wash Factors Evaluation}




\subsection{Sludge Wash Factors Evaluation}

The analytical water wash data, contained in the "WATER" column of the characterization worksheets, provided tank-specific information about the fraction of each component that dissolves with water, i.e., an estimate of tank-specific wash factors for evaluating tank-by-tank processing. The wash factors tend to vary from tank to tank, a reflection of the differences in elemental inventories as well the differences in chemical species. An important point to note is that wash factors derived from the analytical data may be misleading for some components. When analyses are performed solely on the water-digested portion of the sludge, as is the case with many anions, the wash factors for these components appear as $100 \%$. However, qualitative analysis by scanning electron microscopy indicates fluorine and sulfur may be present in some washed sludge samples. Because of their potential impact on $\mathrm{HLW}$ vitrification, the partitioning behavior of anions, such as $\mathrm{F}^{-}, \mathrm{SO}_{4}{ }^{2-}$, and $\mathrm{Cl}^{-}$, needs to be better established.

The characterization wash data can be used collectively to evaluate some of the wash factors that are assumed for the overall SST waste inventory; specifically, wash factors for elements that would be found primarily in sludges. For example, tank wastes listed in Table 2.1 represent approximately $40 \%$ of the SST sludge-type wastes. If the bulk of an element is expected to be in sludge-type wastes, e.g., bismuth, then a wash factor based on this $40 \%$ sludge inventory might be expected to be similar to the wash factor assumed for the overall SST waste inventory. As shown in Table 3.1, which contains wash factors calculated from sludge characterization data, the calculated wash factor for bismuth shows that less than $1 \%$ of the bismuth inventory is removed with washing. The currently assumed wash factor for bismuth for the overall SST inventory is $25 \%$ (see Table 3.2). Table 3.2 provides calculated wash factors, for selected elements, as compared with current wash factors assumed for systems engineering studies. 
Table 3.1. Calculated Sludge Wash Factors Based on Characterization Data

\begin{tabular}{|c|c|c|c|c|c|c|c|c|c|c|c|c|c|c|}
\hline Tank ID & $\begin{array}{c}\text { Al Total } \\
\text { (kg) }\end{array}$ & $\begin{array}{c}\text { A) Wash } \\
\text { (kg) }\end{array}$ & $\begin{array}{c}\text { Ba Total } \\
\text { (kg) }\end{array}$ & $\begin{array}{c}\begin{array}{c}\text { Ba Wash } \\
\text { (kg) }\end{array} \\
\end{array}$ & $\begin{array}{c}\text { BI Total } \\
(\mathrm{kg})\end{array}$ & $\begin{array}{r}\text { BI Wash } \\
(\mathbf{k g})\end{array}$ & $\begin{array}{r}\text { B Total } \\
(\mathrm{kg})\end{array}$ & $\begin{array}{r}\text { B Wash } \\
(\mathrm{kg})\end{array}$ & $\begin{array}{c}\text { Cd Total } \\
\text { (kg) }\end{array}$ & $\begin{array}{r}\text { Cd Wash } \\
\text { (kg) }\end{array}$ & $\begin{array}{c}\text { Ca Total } \\
(\mathrm{kg})\end{array}$ & $\begin{array}{r}\text { Ca Wash } \\
(\mathrm{kg})\end{array}$ & $\begin{array}{c}\text { Cr Total } \\
(\mathbf{k g})\end{array}$ & $\begin{array}{r}\text { Cr Wash } \\
\text { (kg) }\end{array}$ \\
\hline$A-102$ & 5181 & 2661 & 196 & 1 & 387 & 15 & 3 & 3 & 14 & 3 & 577 & 19 & 1290 & 29 \\
\hline A-103 & 30874 & 27669 & 1072 & 8 & 329 & 161 & 41 & 41 & 169 & 129 & 3196 & 262 & 2852 & 85 \\
\hline A-106 & 16713 & 7561 & 1578 & 2 & 171 & 36 & 14 & 14 & 28 & 8 & 3864 & 47 & 3713 & 303 \\
\hline B-110 & 1535 & 0 & 31 & $<\mathrm{DL}$ & 25190 & 53 & 43 & 5 & 38 & 8 & 1211 & 42 & 1217 & 88 \\
\hline B-111 & 1519 & 0 & 47 & $\angle D L$ & 22544 & 54 & 76 & 16 & 22 & $<D L$ & 762 & 10 & 1279 & 298 \\
\hline B-201 & 643 & 7 & 53 & 0.1 & 13700 & 2 & 9 & 1 & 5 & 0.1 & 1824 & 5 & 443 & 114 \\
\hline BX-104 & 31085 & 784 & 1079 & 1 & 919 & 7 & 7 & 4 & 16 & 2 & 3178 & 39 & 2484 & 388 \\
\hline BX-105 & 9940 & 419 & 938 & 1 & 228 & 4 & 2 & 2 & 13 & 1 & 1823 & 26 & 2537 & 44 \\
\hline$B X-107$ & 22382 & 209 & 14 & 1 & 37733 & 365 & 73 & 39 & 7 & 1 & 1622 & 677 & 1513 & 236 \\
\hline C-103 & 4545 & 3 & 1555 & 1 & 226 & 21 & 1 & 0 & 160 & 2 & 3481 & 12 & 193 & 5 \\
\hline C.104 & 40722 & 731 & 5277 & 4 & 4991 & 81 & 23 & 4 & 1731 & 89 & 15229 & 30 & 1513 & 61 \\
\hline C-105 & 54422 & 90 & 2256 & 3 & 550 & 62 & 11 & 3 & 139 & 50 & 6864 & 63 & 830 & 412 \\
\hline C-106 & 43633 & 23 & 5210 & 3 & 534 & 16 & 21 & 5 & 395 & 13 & 12715 & 49 & 1050 & 2 \\
\hline C-109 & 34266 & 73 & 18 & $<D L$ & & & 26 & 6 & 3 & $<D L$ & 5565 & 37 & 69 & 49 \\
\hline C-110 & 12367 & 1028 & 6 & 1 & 14014 & 100 & 21 & 21 & 4 & 1 & 540 & 159 & 396 & 221 \\
\hline C-112 & 10688 & 333 & 51 & $<D L$ & & & 75 & 20 & 3 & $<D L$ & 14575 & 185 & 148 & 94 \\
\hline S-104 & 196294 & 7871 & 55 & 1 & $<D L$ & & 65 & 15 & $<\mathrm{DL}$ & & 3183 & 164 & 7132 & 3184 \\
\hline$T-102$ & 38840 & 102 & 2 & $<D L$ & $<D L$ & & & & 2 & $<D L$ & 95 & 2 & 101 & 99 \\
\hline$T-104$ & 34467 & 298 & 17 & 1 & 36314 & 321 & 27. & 22 & 11 & $<D L$ & 1277 & 353 & 1762 & 278 \\
\hline$T-107$ & 15864 & 632 & & & 11648 & 237 & 13 & 13 & & & 738 & 263 & 349 & 205 \\
\hline$T-111$ & 1075 & 21 & 130 & 1 & 48943 & 381 & 61 & 8 & 15 & 1. & 4569 & 116 & 3725 & 412 \\
\hline TY-101 & 21270 & 29 & 1411 & 0.3 & 19942 & 6 & & & 8 & 1 & & & 6156 & 311 \\
\hline$T Y-103$ & 8560 & 4 & 522 & 7.4 & 28575 & 7 & & & 8 & 2 & & & 1045 & 423 \\
\hline TY-104 & 2500 & 114 & 122 & 0.3 & 4883 & 3 & & & 3 & 1 & & & 636 & 493 \\
\hline TY-105 & 2558 & 3 & 67 & 4 & 522 & 10 & & & 7 & 2 & & & 177 & 31 \\
\hline$T Y-106$ & 604 & 0.3 & 115 & 1 & 46 & 1 & & & 3 & 0.2 & & & 13 & 0.1 \\
\hline U-110 & 177565 & 2815 & 55 & 6 & 14731 & $<D L$ & 301 & 301 & 17 & $<\mathrm{DL}$ & 3005 & 115 & 413 & 245 \\
\hline & 820112 & 53480.3 & 21877 & 48.1 & 287120 & 1943 & 913 & 543 & 2821 & 314.3 & 89893 & 2675 & 43036 & 8110.1 \\
\hline $\begin{array}{l}\text { \% SST Inv (a) } \\
\text { Wash Factor }\end{array}$ & 31 & 6.52 & & 0.22 & 110 & 0.68 & & 59.47 & 73 & 11.14 & 70 & 2.98 & 45 & 18.84 \\
\hline
\end{tabular}


Table 3.1. Contd.

\begin{tabular}{|c|c|c|c|c|c|c|c|c|c|c|c|c|c|c|}
\hline Tank ID & $\begin{array}{c}\text { Co Total } \\
(\mathrm{kg})\end{array}$ & $\begin{array}{r}\text { Co Wash } \\
(\mathrm{kg})\end{array}$ & $\begin{array}{c}\text { Cu Total } \\
(\mathrm{kg})\end{array}$ & $\begin{array}{r}\text { Cu Wash } \\
(\mathbf{k g})\end{array}$ & $\begin{array}{c}\text { Fe Total } \\
(\mathrm{kg})\end{array}$ & $\begin{array}{r}\text { Fe Wash } \\
\text { (kg) }\end{array}$ & $\begin{array}{r}\text { La Total } \\
(\mathrm{kg})\end{array}$ & $\begin{array}{r}\text { La Wash } \\
(\mathbf{k g})\end{array}$ & $\begin{array}{c}\text { Pb Total } \\
(\mathrm{kg})\end{array}$ & $\begin{array}{r}\text { Pb Wash } \\
(\mathbf{k g})\end{array}$ & $\begin{array}{c}\text { Mg Total } \\
\text { (kg) }\end{array}$ & $\begin{array}{c}\text { Mg Wash } \\
(\mathbf{k g})\end{array}$ & $\begin{array}{c}\text { Mn Total } \\
\text { (kg) }\end{array}$ & $\begin{array}{c}\begin{array}{c}\text { Mn Wash } \\
(\mathrm{kg})\end{array}\end{array}$ \\
\hline A-102 & $\frac{\operatorname{lng} /}{6}$ & $\mathbf{l}^{1} \mid$ & $\frac{\ln 19}{19}$ & में 1 & $\frac{3 y}{3103}$ & $\begin{array}{r}\text { (ng) } \\
1\end{array}$ & & & 264 & $\begin{array}{r}\text { (n) } 11 \\
\end{array}$ & 308 & $\begin{array}{r}\text { my) } \\
0.3\end{array}$ & $\frac{\ln }{479}$ & $\begin{array}{r}27 \\
27\end{array}$ \\
\hline A-103 & 3 & 0 & 25 & 6 & 661 & 12 & & & 678 & 482 & 1482 & 5 & 232 & 53 \\
\hline A-106 & 10 & 3 & 61 & 3 & 19326 & 3 & & & 791 & 27 & 2079 & 0.4 & 1103 & 66 \\
\hline B-110 & $<D L$ & & 74 & 35 & 24181 & 149 & & & 823 & $<\mathrm{DL}$ & 239 & 5 & 113 & $<D L$ \\
\hline B-111 & 18 & $<D L$ & 247 & 5 & 18267 & 91 & & & 2062 & $<D L$ & 824 & $\angle D L$ & 124 & $<D L$ \\
\hline B-201 & 9 & 0.1 & 16 & 0.1 & 2001 & 1 & 2040 & 3 & 200 & 1 & 452 & 2 & 3058 & 0.5 \\
\hline$B X-104$ & 8 & 0 & 63 & 5 & 4697 & 1 & & & 375 & 7. & 1621 & 1 & 643 & 14 \\
\hline$B X-105$ & 0.2 & 0.2 & 8 & 1 & 1834 & 1 & & & 165 & 13 & 955 & 1 & 438 & 8 \\
\hline$B X-107$ & 11 & 2 & 81 & 1 & 17292 & 185 & & & 116 & 12 & 248 & 26 & 101 & 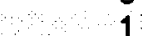 \\
\hline $\mathrm{C}-103$ & 20 & 11 & 268 & 5 & 27150 & 2 & & & 1116 & 8 & 1940 & 3 & 776 & 4 \\
\hline C-104 & 22 & D & 151 & 4 & 25231 & 9 & & & 1347 & 243 & 7382 & 6 & 4456 & 27 \\
\hline C-105 & 9 & 0 & 136 & 0 & 10605 & 5 & & & 790 & 187 & 3250 & 21 & 2176 & 21 \\
\hline C-106 & 5 & 0 & 137 & 2 & 55511 & 1 & & & 2564 & 48 & 6994 & 12 & 1963 & 5 \\
\hline C-109 & $<D L$ & & 20 & $<D L$ & 5334 & 253 & & & 1333 & 7 & 138 & 2 & 35 & $<\mathrm{DL}$ \\
\hline C.110 & 5 & 2 & 70 & 2 & 9302 & 263 & & & 227 & 17 & 127 & $1 \overline{2}$ & 45 & 2 \\
\hline C-112 & $\angle D L$ & & 34 & $<\mathrm{DL}$ & 14425 & 893 & & & 1293 & $<\mathrm{DL}$ & 332 & 22 & 139 & 1 \\
\hline S-104 & 11 & & 86 & 1. & 4136 & 5 & & & 64 & 14 & 89 & 7 & 1821 & 0.5 \\
\hline T-102 & $<\mathrm{DL}$ & & 7 & $<\mathrm{DL}$ & 2600 & 13 & & & 54 & $<D L$ & 14 & $<D L$ & 125 & 1 \\
\hline$T-104$ & 21 & $<\mathrm{DL}$ & 90 & $<\mathrm{DL}$ & 17344 & 153 & & & 118 & $<\mathrm{DL}$ & 307 & 12 & 115 & 1 \\
\hline T-107 & & & & & 30558 & 345 & & & 773 & 40 & 220 & 31 & 215 & 3 \\
\hline$T-111$ & 22 & 2 & 63 & 1 & 34892 & 241 & 7948 & 21 & 688 & 13 & 711 & 7 & 11939 & 47 \\
\hline TY-101 & & & & & 29411 & 39 & & & 164 & $\begin{array}{r}5 \\
5\end{array}$ & & & 409 & $\begin{array}{l}41 \\
11\end{array}$ \\
\hline$T Y-103$ & & & & & 24815 & 163 & & & 488 & 5 & & & 167 & 13 \\
\hline TY-104 & & & & & 8959 & 24 & & & 159 & 3 & & & 575 & 4 \\
\hline TY-10S & & & & & 27959 & 1 & & & 519 & 7 & & & & \\
\hline TY-106 & & & & & 4037 & 0.4 & & & 34 & 1 & & & 49 & 2 \\
\hline $0-110$ & & & 304 & $<D L$ & 10643 & 34 & & & 519 & 60 & 1436 & 335 & 2490 & $<D L$ \\
\hline & 180.2 & 23.3 & 1960 & 72.1 & 434274 & 2888.4 & 9988 & 24 & 17724 & 1211 & 31148 & 5107 & 33786 & 312 \\
\hline $\begin{array}{l}\text { \% SST Inv (a) } \\
\text { Wash Factor }\end{array}$ & & 12.93 & & 3.68 & 59 & 0.67 & & 0.24 & 4 & 6.83 & & 1.64 & 28 & 0.92 \\
\hline
\end{tabular}


Table 3.1. Contd.

\begin{tabular}{|c|c|c|c|c|c|c|c|c|c|c|c|c|c|c|}
\hline Tank ID & $\begin{array}{c}\begin{array}{c}\text { Ni Total } \\
(\mathbf{k g})\end{array} \\
\end{array}$ & $\begin{array}{r}\text { NI Wash } \\
\text { (kg) }\end{array}$ & $\begin{array}{l}\text { P04 Total } \\
\text { (kg) }\end{array}$ & $\begin{array}{r}\text { P04 Wash } \\
\text { (kg) }\end{array}$ & $\begin{array}{c}\text { K Total } \\
(\mathrm{kg})\end{array}$ & $\begin{array}{r}\text { K Wash } \\
\text { (kg) }\end{array}$ & $\begin{array}{r}\text { SI Total } \\
(\mathrm{kg})\end{array}$ & $\begin{array}{r}\text { SI Wash } \\
\text { (kg) }\end{array}$ & $\begin{array}{c}\text { Ag Total } \\
(\mathrm{kg})\end{array}$ & $\begin{array}{r}\text { Ag Wash } \\
(\mathrm{kg})\end{array}$ & $\begin{array}{r}\text { Na Total } \\
(\mathbf{k g})\end{array}$ & $\begin{array}{r}\text { Na Wash } \\
\text { (kg) }\end{array}$ & $\begin{array}{c}\text { Sr Total } \\
\text { (kg) }\end{array}$ & $\begin{array}{r}\text { St Wash } \\
(\mathrm{kg})\end{array}$ \\
\hline A-102 & 117 & 3 & 3499 & 2979 & 627 & 542 & 3681 & 29 & 55 & 1 & 41650 & 38411 & 22 & 0.1 \\
\hline A-103 & 174 & 69 & 12132 & 11906 & 4721 & 4397 & 20585 & 714 & 46 & 4 & 388682 & 380101 & 22 & 0 \\
\hline A-106 & 458 & 9 & 38532 & 36521 & 1647 & 1243 & 28143 & 56 & 207 & 3 & 89321 & 74911 & 39 & 0.3 \\
\hline B-110 & 111 & $\angle D L$ & 65417 & 29369 & 585 & 388 & 12337 & 471 & 109 & 10 & 125366 & 113717 & 271 & $<D L$ \\
\hline B-111 & 21 & $\angle D L$ & 50891 & 25187 & 685 & 685 & 10763 & 730 & 109 & $<D L$ & 97648 & 89737 & 247 & 1 \\
\hline B-201 & 99 & 0.4 & 2408 & 171 & 793 & 603 & 3222 & 88 & 8 & 0.1 & 5502 & 4062 & 123 & 0.1 \\
\hline$B \times-104$ & 102 & 11 & 7521 & 6287 & 692 & 329 & 21238 & 86 & 59 & 1 & 45025 & 32301 & 41 & 0 \\
\hline BX-105 & 43 & 8 & 17529 & 13545 & 422 & 230 & 12165 & 210 & 8 & 0.4 & 32286 & 23265 & 17 & 0.2 \\
\hline$B X-107$ & 19 & 1 & 110035 & 22452 & 411 & 231 & 10597 & 459 & 9 & 2 & 158198 & 106403 & 263 & 4 \\
\hline$c-103$ & 891 & 14 & 3925 & 718 & 440 & 54 & 22124 & 29 & 69 & 4 & 15822 & 2291 & 37 & 0 \\
\hline C-104 & 2581 & 105 & 12666 & 353 & 1820 & 469 & 76186 & 2891 & 634 & 2 & 129026 & 78442 & 110 & 0 \\
\hline$c-105$ & 1884 & 5 & 6687 & 3485 & 974 & 339 & 35079 & 43 & 59 & 1 & 63159 & 30721 & 224 & 0 \\
\hline C-106 & 1038 & 34 & 9309 & 1407 & 1573 & 166 & 75718 & 26 & 564 & 1 & 124871 & 48494 & 110 & 0 \\
\hline C-109 & 4239 & 25 & 16506 & 4735 & 146 & 138 & 2544 & 26 & $<D L$ & & 23718 & 17979 & 52 & 0.3 \\
\hline$C-110$ & 21 & 2 & 52541 & 20420 & 482 & 337 & 5991 & 204 & 4 & 1 & 71091 & 56200 & 107 & 5 \\
\hline C-112 & 9839 & 529 & 52008 & 20220 & 369 & 320 & 1503 & 43 & $<D L$ & & 69565 & 62971 & 188 & 2 \\
\hline s-104 & 8123 & 3 & 481 & 45 & 499 & 421 & 2429 & 57 & $<D L$ & & 198512 & 151185 & 696 & 1 \\
\hline$T-102$ & 9 & $\angle D L$ & 592 & 160 & $\angle D L$ & & 417 & 6 & 19 & $<D L$ & 3709 & 3709 & 2 & $<D L$ \\
\hline$T-104$ & 24 & $\angle D L$ & 142274 & 36968 & 165 & 72 & 12390 & 331 & $<D L$ & & 124045 & 89462 & 190 & 2 \\
\hline$T-107$ & 2561 & 9 & 93420 & 69100 & 306 & 306 & 5881 & 1923 & & & 126454 & 104755 & 934 & 5 \\
\hline$T-111$ & 248 & 3 & 58958 & 32139 & 2141 & 1357 & 10689 & 1078 & 241 & 1 & 69737 & 62146 & 562 & 4 \\
\hline TY-101 & 3729 & 56 & 54369 & 11844 & & & 28331 & 236 & 3 & 1 & 88849 & 54935 & & \\
\hline$T Y-103$ & 2641 & 66 & 65554 & 19732 & & & 9805 & 76 & 9 & 1 & 110410 & 77033 & & \\
\hline TY-104 & 427 & 5 & 21614 & 14669 & & & 2226 & 45 & 3 & 1 & 31119 & 23696 & & \\
\hline TY.105 & 114 & 1 & 155792 & 10515 & & & 492 & 92 & 2 & 1 & 154241 & 109427 & & \\
\hline TY-106 & & 0.1 & 5099 & 1107 & & & 7840 & 5 & 2 & 0.1 & 9316 & 7017 & & \\
\hline $0-110$ & 3766 & $\angle D L$ & 46338 & 46338 & 1278 & 600 & 11532 & 869 & 71 & CDL & 114616 & 69464 & 425 & 4 \\
\hline & 43285 & 958.5 & 1106097 & 442372 & 20776 & 13227 & 433908 & 10823 & 2290 & 35.6 & 2511938 & 1912835 & 4682 & 29 \\
\hline $\begin{array}{l}\text { \% SST Inv (a) } \\
\text { Wash Factor }\end{array}$ & 24 & 2.21 & 24 & 39.99 & 35 & 63.66 & 97 & 2.49 & & 1.55 & 4 & 76.15 & 13 & 0.62 \\
\hline
\end{tabular}


Table 3.1. Contd

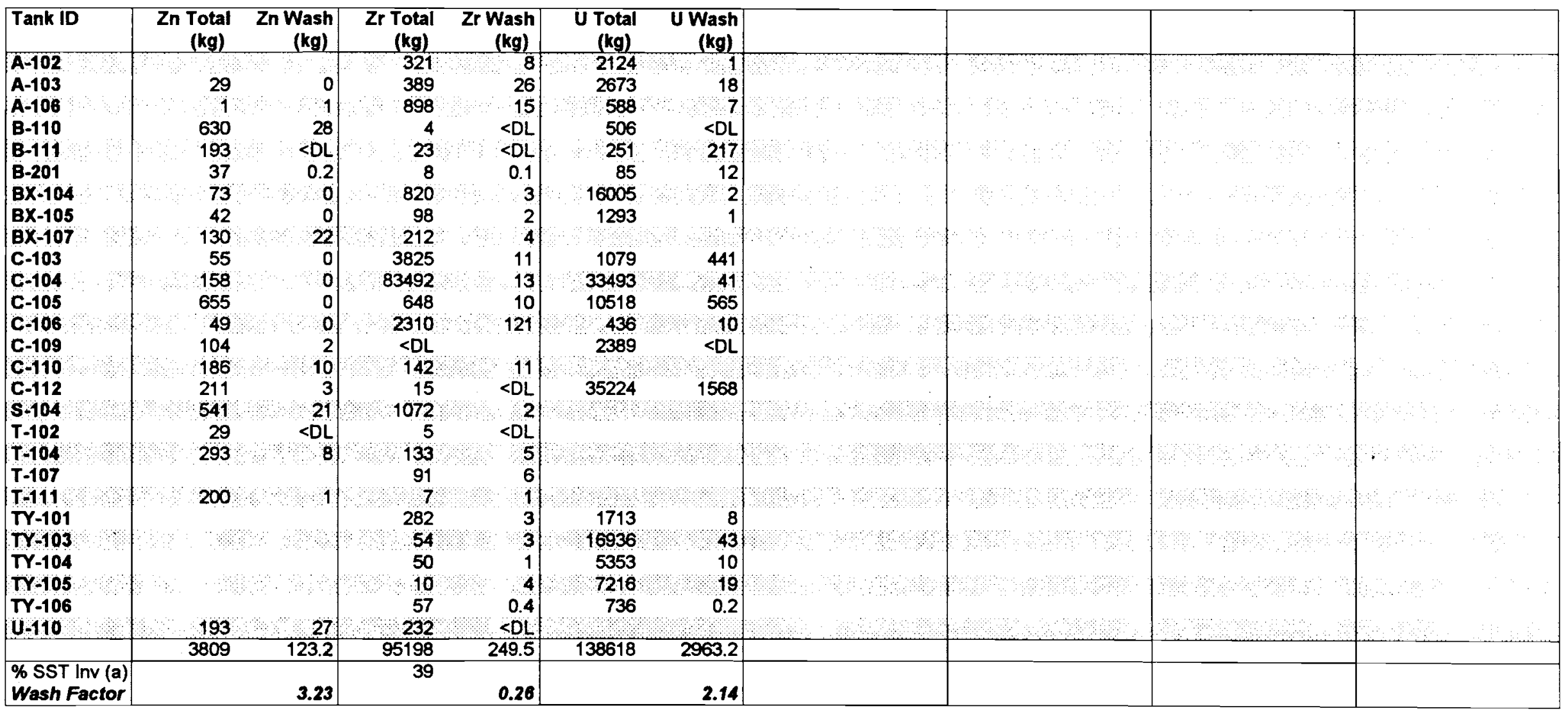

(a) Chemical inventories based on Stordeur (1986). Reported inventories for $\mathrm{Al}, \mathrm{Cr}$, and PO4 are being investigated by WHC Systems Engineering group. 
Table 3.2. Calculated vs. Assumed Wash Factors (percent soluble in wash)

\begin{tabular}{|c|c|c|c|}
\hline & Assumed ${ }^{(\boldsymbol{a})}$ & Calculated & $\begin{array}{l}\text { \% SST Inv } \\
\text { Calculation } \\
\text { Based On }\end{array}$ \\
\hline $\mathrm{Ag}^{+}$ & 0 & 2 & \\
\hline$* \mathrm{Al}^{+3}$ & 25 & 7 & 31 \\
\hline $\mathrm{B}^{+3}$ & 0 & 59 & \\
\hline $\mathrm{Ba}^{+2}$ & 0 & $<1$ & \\
\hline $\mathrm{Bi}^{+3}$ & 25 & $<1$ & 110 \\
\hline $\mathrm{Ca}^{+2}$ & 5 & 3 & 70 \\
\hline $\mathrm{Cd}^{+2}$ & 50 & 11 & 73 \\
\hline $\mathrm{Co}^{+3}$ & 0 & 13 & \\
\hline$* \mathrm{Cr}^{+3 /+6}$ & 10 & 19 & 45 \\
\hline $\mathrm{Cu}^{+2}$ & 0 & 4 & \\
\hline $\mathrm{Fe}^{+3}$ & 1 & $<1$ & 59 \\
\hline $\mathrm{K}^{+}$ & 0 & 64 & 35 \\
\hline $\mathrm{La}^{+3}$ & 1 & $<1$ & \\
\hline $\mathrm{Mg}^{+2}$ & 0 & 2 & \\
\hline $\mathrm{Mn}^{+2 /+3 /+4}$ & 5 & $<1$ & 28 \\
\hline $\mathrm{Ni}^{+3}$ & 0 & 2 & 24 \\
\hline $\mathrm{Pb}^{+2}$ & 0 & 7 & 4 \\
\hline$* \mathrm{PO}_{4}^{-3} / \mathrm{P}_{2} \mathrm{O}_{7}^{-4}$ & 50 & 40 & 24 \\
\hline $\mathrm{Si}^{+4}$ & 0 & 2 & 97 \\
\hline $\mathrm{Sr}^{+2}$ & 1 & $<1$ & 13 \\
\hline $\mathrm{UO}_{2}^{+2}$ & 5 & 2 & \\
\hline $\mathrm{Zn}^{+2}$ & 0 & 3 & \\
\hline $\mathrm{Zr}^{+4}$ & 0 & $<1$ & 39 \\
\hline
\end{tabular}

* Note that these may be primary constituents in salt cake- as well as sludge-type wastes.

(a) Boomer, et al. (1993).

(b) Stordeur, et al. (1986). 
4.0 Spreadsheet Evaluation of the Enhanced Sludge Wash Process 


\subsection{Spreadsheet Evaluation of the Enhanced Sludge Wash Process}

A spreadsheet was developed to evaluate the effect of enhanced sludge washing on tankspecific sludges and different waste types. Input to the spreadsheet originated from the characterization data and consisted of 1) component concentrations prior to any treatment and 2) component concentrations that were reported to be removed with water washes. Output from the spreadsheet consisted of the estimated mass and percentage of each element that would be removed with washing and leaching and the estimated compositions, both in terms of concentration and dry weight percent, of the final wash and leach streams and the residual solids.

\subsection{Assumptions}

For this evaluation, enhanced sludge washing was defined as a $0.1 \underline{\mathrm{M}} \mathrm{NaOH}$ wash followed by a $3 \mathrm{M} \mathrm{NaOH}$ leach, and the following assumptions were made:

- Cation-to-anion ratios are balanced within experimental error. (Note, this is not necessarily true for all characterized wastes; e.g., in C-106, the sodium concentration appears too high. This excess sodium shows up in the wash and is reflected in the sodium wash factor).

- Separation of liquid and solid phases is ideal.

- Mass of water removed from sludge at a particular step is proportional to mass of solids removed from sludge at that step.

- Volume wash or leachate out $=$ volume wash or leachate in + water removed from sludge

- Sodium from the wash or leachate remains in the wash or leachate.

- Calcium and magnesium not removed in the wash are tied to phosphorus in a 3:2 mole ratio and will not leach with caustic.

- 4 volumes dilute caustic wash:1 volume sludge.

- With the exception of anions, tank-specific characterization wash factors provide a reasonable estimate of pretreatment wash factors. When anion inventories are based solely on analyses performed on the water-digested portion of the sludge, wash factors for these components are assumed to be $\leq 100 \%$. In cases where characterization wash data were reported as "less than detection limit," wash factors are assumed to be $\geq 0 \%$.

- Only aluminum, chromium, and phosphorus leach with caustic:

- Water-insoluble phosphorus that is not tied to calcium will leach with caustic.

- Aluminum and silicon not removed in the wash are tied up at a 1:1 mole ratio; 85\% of excess aluminum will leach with caustic.

- 75\% of water-insoluble chromium will leach with caustic.

These leaching assumptions are consistent with current systems engineering assumptions. To date, no assumptions have been made as to the leaching behavior of components such as sodium, silicon, zinc, chloride, fluoride, sulfate, and the radionuclides. 
- Calculated volume of caustic leach is based on enough $\mathrm{NaOH}$ (up to $3 \underline{\mathrm{M}}$ free hydroxide) to provide:

3 moles $\mathrm{OH}^{*}: 1$ mole $\mathrm{PO}_{4}{ }^{3-}$ (after wash)

1 mole $\mathrm{OH}^{-}: 1$ mole $\mathrm{Cr}$ [after wash, assumes $\mathrm{Cr}$ exists as $\mathrm{Cr}(\mathrm{OH})_{3}$ ]

1 mole $\mathrm{OH}: 1$ mole $\mathrm{Al}$ [after wash, assumes $\mathrm{Al}$ exists as $\mathrm{Al}(\mathrm{OH})_{3}$ ].

\subsection{Verification}

Characterization data for five core composites were evaluated with the enhanced sludge washing spreadsheet to verify spreadsheet performance. The results from these evaluations were compared with results from recent washing and leaching experiments performed with samples from these same core composites. Selected results, in terms of percent element removed, are summarized in Table 4.1. The data summaries from the spreadsheet evaluation of the five core composites are provided at the back of this section.

Table 4.1. Calculated vs. Experimental Enhanced Sludge Washing Results

\begin{tabular}{|c|c|c|c|c|c|c|c|c|c|c|}
\hline \multicolumn{11}{|c|}{ \% Component Dissolved with Enhanced Sludge Washing } \\
\hline & \multicolumn{2}{|c|}{$\begin{array}{l}\text { B-110 } \\
\text { Core } 1\end{array}$} & \multicolumn{2}{|c|}{$\begin{array}{c}\text { C-109 } \\
\text { Core } 47\end{array}$} & \multicolumn{2}{|c|}{$\begin{array}{c}\text { C-112 } \\
\text { Core } 36\end{array}$} & \multicolumn{2}{|c|}{$\begin{array}{c}\text { U-110 } \\
\text { Core } 14\end{array}$} & \multicolumn{2}{|c|}{$\begin{array}{c}\text { B-201 } \\
\text { Core } 27\end{array}$} \\
\hline & $\exp ^{(a)}$ & cal & $\exp ^{(\mathbf{a})}$ & cal & $\exp ^{(a)}$ & cal & $\exp ^{(\mathbf{a}, \mathbf{b})}$ & cal & $\exp ^{(c)}$ & cal \\
\hline Al & $<53$ & 0 & 81 & 74 & 85 & 63 & 79 & 80 & $18-41$ & 1 \\
\hline $\mathbf{C a}$ & 19 & 9 & 1 & 1 & 1 & 2 & 52 & 3 & 0 & 0 \\
\hline $\mathbf{C r}$ & 64 & 77 & $84-75$ & 91 & 88 & 90 & $74-47$ & 94 & $48-65$ & 82 \\
\hline $\mathbf{F e}$ & 0 & 1 & 5 & 3 & 5 & 5 & 1 & 0 & 1 & 0 \\
\hline $\mathbf{P}$ & 97 & 97 & 42 & 35 & 84 & 70 & $49(51)^{(d)}$ & 100 & $16-39$ & 44 \\
\hline $\mathbf{S i}$ & 74 & 4 & 16 & 1 & 11 & 4 & 8 & $15^{(e)}$ & $36-55$ & 3 \\
\hline
\end{tabular}

(a) Lumetta, et al. (1994).

(b) Experimental results for U-110 are for a sample treated with a $5 \underline{\mathrm{M}} \mathrm{NaOH}$ leach followed a $\mathrm{K}_{2} \mathrm{CO}_{3}$ wash.

(c) Lumetta and Rapko (1994).

(d) $49 \%$ of the phosphate was removed with $5 \underline{\mathrm{M}} \mathrm{NaOH} ; 51 \%$ of the phosphate was subsequently removed with a $\mathrm{K}_{2} \mathrm{CO}_{3}$ wash.

(e) No reported value for total silicon in Core 14; total silicon estimated based on Core 7.

The information provided by the comparison of spreadsheet calculations and experimental data is discussed below for several individual elements. 
Aluminum. Calculated dissolution values, based on the assumption that $85 \%$ of excess aluminum will leach with caustic, compare favorably with experimental values. In most cases, the estimated values appear to be more conservative than the experimental values. In the experimental studies, some of the aluminum that is tied to silicon may have dissolved with caustic leaching, and/or some of these species may have been in the form of colloids that did not readily settle out of solution. The spreadsheet evaluation assumes ideal separation of liquid and solid phases and does not account for dissolution of any aluminum that is tied to silicon.

Calcium. Calculated dissolution values, based on the assumption that calcium not removed in the wash is tied to phosphorus in a 3:2 mole ratio and will not leach, compare favorably with experimental values for wastes from Tanks C-109, C-112, and B-201. The differences between the calculated and experimental values for wastes from B-110 and U-110 may occur because some of the calcium species in these wastes may have been in the form of colloids that did not settle out of solution in experimental studies. Also, the total concentrations for sludge samples in earlier experimental studies were obtained by summing the fractions of the wash, leach, and residual solids portions. If reported analytical concentrations for any fraction were less than or greater than actual concentrations, the percent dissolved values would reflect these discrepancies. Also, wastes from B-110 and U-110 contained lower calcium concentrations per gram of sludge than the other wastes.

Chromium. Calculated dissolution values, based on the assumption that $75 \%$ of water-insoluble chromium will leach with caustic, compare reasonably (within a factor of $1.1-1.2$ ) with experimental values. However, in most cases, the calculated dissolution value is greater than the experimental.

Iron. Calculated dissolution values compare favorably with experimental values.

Phosphorus. Calculated dissolution values, based on the assumption that water-insoluble phosphorus not tied to calcium will leach with caustic, compare favorably with experimental values. However, several important points should be noted. First, some phosphorus that may be tied to bismuth may metathesize with the $0.1 \mathrm{M} \mathrm{NaOH}$ wash. Bismuth phosphate has been identified in Tank $\mathrm{B}-110$ by $\mathrm{X}$-ray diffraction analysis, and experimental studies showed that approximately $84 \%$ of the phosphate in this waste was removed with the dilute caustic wash and an additional $13 \%$ of the phosphate was removed with the caustic leach. On the other hand, wash factors for the spreadsheet are based on water wash characterization data, and water does not metathesize bismuth phosphate. Therefore, the spreadsheet shows only $45 \%$ of the phosphate is removed with the wash, and an additional $52 \%$ of the phosphate is removed with the alkaline leach. As a result, the calculated volume of leachate on the spreadsheet is higher than will actually be needed to pretreat this waste.

Note that all tanks that contain bismuth and phosphate do not necessarily contain bismuth phosphate. In tanks where the $\mathrm{pH}$ was originally high, e.g., $\mathrm{pH}$ of 13 , the bismuth may have already metathesized to a bismuth oxide species. Also, wastes that contain significant concentrations of fluoride, e.g., $7000 \mu \mathrm{g} \mathrm{F/g} \mathrm{sludge} \mathrm{as} \mathrm{in} \mathrm{waste} \mathrm{from} \mathrm{Tank} \mathrm{U-110,} \mathrm{may} \mathrm{contain} \mathrm{the} \mathrm{double} \mathrm{salt} \mathrm{sodium}$ fluoride phosphate $\left[\mathrm{Na}_{7} \mathrm{~F}\left(\mathrm{PO}_{4}\right)_{2} \cdot \mathrm{xH}_{2} \mathrm{O}\right]$. Because of the seven sodiums, the solubility of this salt may decrease with increased sodium concentrations. The results in Table 4.1 show that only half of the 
phosphate was removed from U-110 sludge with $5 \underline{\mathrm{M}} \mathrm{NaOH}$, while almost all the phosphate was removed with water.

Silicon. Calculated dissolution values, based on the assumption that no silicon leaches with caustic, do not compare favorably with experimental results. The discrepancies most likely occur because some silicon leaches with caustic, and some of the silicon may be in the form of colloids that do not readily settle out of solution.

\subsection{Results}

After the spreadsheet results were compared with experimental results, the spreadsheet was used to evaluate the effect of enhanced sludge washing on individual tank sludges, as well as for groups of sludges that may be representative of different waste types.

\subsubsection{Specific Tank Sludges}

Only sludges with $\geq 90$ dry weight percent solids identified (refer to Table 2.1) were included in this evaluation, and tank compositions were derived by averaging core composites for a particular tank. The data summaries from these evaluations are provided at the end of this section. These summaries provide the estimated mass and cumulative percentage of each element that would be removed with washing and leaching and the estimated compositions, both in terms of concentration and dry weight percent, of the final wash and leach streams and the residual solids. The residual solids information may be used to evaluate the impact of not blending any tank sludges during pretreatment processing and to assess which tank sludges might be blended together. Note that wash factors based on averaged core composites may differ from wash factors for a specific core.

\subsubsection{Different Waste Types}

In this evaluation, sludges were grouped according to waste type. This classification method is based on the assumption that the primary and secondary solids-forming waste types in a tank are responsible for the bulk chemical composition and physical properties of the sludge contained in that tank. Waste types for each tank were identified by Hill and Simpson (1994) with the Sort On Radioactive Waste Type (SORWT) model. Table 4.2 lists, for each sludge group, the primary and secondary waste types; the specific tanks that comprise a group; and the total volume of waste. Available tank-specific compositional data for each group were averaged (mass-weighted basis) to form a representative composition for that particular group, and the representative sludges were evaluated with the spreadsheet.

Data summaries that include wash factors are provided at the end of this section for the waste types listed on the following page. Note that these waste-type wash factors based on mass-weightedaveraged tank compositions may differ slightly from wash factors for individual tanks that belong to a waste-type group. 
Waste Type

Aluminum Cladding Waste

Aluminum Cladding Waste - Mix

$1^{\text {st }}$ Cycle $\mathrm{BiPO}_{4}$

$1^{\text {at }}$ Cycle $\mathrm{BiPO}_{4}$ - Aluminum Cladding Waste

$1^{\text {st }}$ Cycle $\mathrm{BiPO}_{4}$ - Uranium Recovery

$1^{\text {th }}$ Cycle $\mathrm{BiPO}_{4}$ - Evaporator Bottoms

$2^{\text {nd }}$ Cycle $\mathrm{BiPO}_{4}$ - Tank 5-6, B-Plant

$2^{\text {nd }}$ Cycle $\mathrm{BiPO}_{4}$ - Concentration Cycle $\mathrm{BiPO}_{4}$

Concentration Cycle $\mathrm{BiPO}_{4}$

PUREX - PUREX Sludge Supernatant

Uranium Recovery - Diatomaceous Earth

Uranium Recovery - Cladding Waste - Portland Cement

Uranium Recovery - $1^{\text {st }}$ Cycle $\mathrm{BiPO}_{4}$ (ferrocyanide scavenged)

Uranium Recovery (ferrocyanide scavenged) - $1^{\text {at }}$ Cycle $\mathrm{BiPO}_{4}$ REDOX
Tanks Providing

Compositional Data

(U-110 Core 8 Composite)

(T-102)

(T-104)

(U-110)

(BX-107, C-110)

(TY-101)

(B-110, B-111)

(T-111)

(B-201)

(C-103, C-106)

(TY-106)

(BX-105)

(TY-103, TY-104)

(C-109, C-112, T-107)

(S-104) 
Table 4.2. Single-shell Tanks Containing Predominantly Sludge-type Wastes

$\begin{array}{lll}\text { KEY: } & \text { CC } & \text { Complexed Concentrate Waste } \\ \text { CW } & \text { Cladding Waste } \\ \text { DSSF } & \text { Double-shell Slurry Feed } \\ \text { EB } & \text { Evaporator Bottom } \\ \text { Evap } & \text { Evaporator Feed } \\ \text { F } & \text { Ferrocyanide scavenged } \\ \text { HS } & \text { Hot Semi Works } \\ \text { IX } & \text { Ion exchange Waste } \\ \text { NC } & \text { Non-complexed Waste }\end{array}$

\begin{tabular}{|c|c|c|}
\hline POS & \\
\hline PSS & \multicolumn{2}{|c|}{ Purex Sludge Supernatant } \\
\hline SRS & \multicolumn{2}{|c|}{ Strontium Recovery Sludge } \\
\hline TBP & \multicolumn{2}{|c|}{ Uranium Recovery } \\
\hline $1 \mathrm{C}$ & \multicolumn{2}{|c|}{ 1st Cycle, $\mathrm{BiPO}_{4}$} \\
\hline 2C & \multicolumn{2}{|c|}{ 2nd Cycle, $\mathrm{BiPO}_{4}$} \\
\hline 224 & \multicolumn{2}{|c|}{ Concentration Cycle, $\mathrm{BiPO}_{4}$} \\
\hline $5-6$ & \multicolumn{2}{|c|}{ Tank 5-6, B Plant } \\
\hline * & \multicolumn{2}{|c|}{$\begin{array}{l}\text { Enhanced Sludge Washing Data } \\
\text { Summary Available for this Group }\end{array}$} \\
\hline kgal & \multicolumn{2}{|c|}{ 2nd Cycle BiPO, } \\
\hline \multirow[t]{3}{*}{$511 *$} & B-110 & $\overline{5-6}$ \\
\hline & B-111 & $5-6$ \\
\hline & B-112 & $5-6$ \\
\hline 370 & B-104 & EB \\
\hline \multirow[t]{3}{*}{$889^{*}$} & $\mathrm{~T}-111$ & 224 \\
\hline & $\mathrm{T}-110$ & 224 \\
\hline & T-112 & 224 \\
\hline
\end{tabular}

\begin{tabular}{|c|c|c|c|c|c|}
\hline kgal & $\begin{array}{l}\text { Cladding Waste } \\
\text { (CW) }\end{array}$ & 2nd Waste & kgal & $\begin{array}{l}\text { 2nd Cycle BiPO, } \\
\text { (2C) }\end{array}$ & 2nd Waste \\
\hline \multirow[t]{3}{*}{$13^{*}$} & U-201 & & $\overline{511} *$ & B-110 & $5-6$ \\
\hline & $\mathrm{U}-202$ & & & B-111 & $5-6$ \\
\hline & U-203 & & & B-112 & $5-6$ \\
\hline \multirow[t]{4}{*}{200} & B-101 & EB & 370 & B-104 & EB \\
\hline & B-102 & EB & & & \\
\hline & B-103 & EB & $889 *$ & $\mathrm{~T}-111$ & 224 \\
\hline & & & & $T-110$ & 224 \\
\hline 423 & $C-102$ & TBP & & $\mathrm{T}-112$ & 224 \\
\hline \multirow[t]{2}{*}{295} & $C-104$ & POS & & Concentration & \\
\hline & & & kgal & BiPO, (224) & 2nd Waste \\
\hline \multirow[t]{5}{*}{$143 *$} & $\mathrm{~T}-101$ & Mix & $276^{*}$ & B-201 & \\
\hline & $\mathrm{T}-102$ & Mix & & B-202 & \\
\hline & $T-103$ & Mix & & B-203 & \\
\hline & & & & B-204 & \\
\hline & 1st Cycle BiPO & & & $\mathrm{T}-201$ & \\
\hline kgal & (1C) & 2nd Waste & & $\mathrm{T}-202$ & \\
\hline \multirow[t]{2}{*}{$442 *$} & T-104 & & & $T-203$ & \\
\hline & & & & T-204 & \\
\hline \multirow[t]{4}{*}{$303 *$} & $U-110$ & $\mathrm{CW}$ & & & \\
\hline & $\mathrm{T}-105$ & $\mathrm{CW}$ & kgal & PUREX & 2nd Waste \\
\hline & $T-106$ & $\mathrm{CW}$ & 11 & $\mathrm{C}-201$ (HS) & \\
\hline & & & & C-202 (HS) & \\
\hline \multirow[t]{5}{*}{$691^{*}$} & BX-107 & TBP & & C-203 (HS) & \\
\hline & $C-110$ & TBP & & C-204 (HS) & \\
\hline & B-106 & TBP & & & \\
\hline & $T-108$ & TBP & $259 *$ & C-103 (SRS) & PSS \\
\hline & & & & C-106 (SRS) & PSS \\
\hline \multirow[t]{5}{*}{$701 *$} & B-107 & EB & & & \\
\hline & B-108 & EB & 28 & A-104 (PSS) & Purex \\
\hline & B-109 & $\mathrm{EB}$ & & & \\
\hline & BX-110 & EB & 19 & $A-105$ & IX \\
\hline & TY-101(F) & EB & & & \\
\hline 275 & $C-107$ & Purex (SRS) & & & \\
\hline
\end{tabular}


Table 4.2. Contd.

\begin{tabular}{|c|c|c|c|c|c|}
\hline kgal & $\begin{array}{l}\text { Uranium Recovery } \\
\text { (TBP) }\end{array}$ & 2nd Waste & kgal & REDOX & 2nd Waste \\
\hline 231 & TY-105 & & $694^{*}$ & $\begin{array}{l}\text { S-104 } \\
\text { SX-107 }\end{array}$ & \\
\hline \multirow{2}{*}{$17 *$} & TY-106 & dia-earth & & SX-108 & \\
\hline & & & & $S X-112$ & \\
\hline \multirow[t]{7}{*}{538} & BX-104 & $\mathrm{CW}$ & & $S X-115$ & \\
\hline & BX-101 & $\mathrm{CW}$ & & TX-101 & \\
\hline & BX-103 & $\mathrm{CW}$ & & U-101 & \\
\hline & BX-106 & $\mathrm{CW}$ & & & \\
\hline & BX-108 & $\mathrm{CW}$ & 368 & SX-110 & Redox IX \\
\hline & BX-109 & $\mathrm{CW}$ & & $S X-111$ & Redox IX \\
\hline & C-101 & $\mathrm{CW}$ & & $S X-114$ & Redox IX \\
\hline \multirow[t]{2}{*}{96} & BX-102 & $\mathrm{CW}$, dia-earth & 777 & S-101 & $\mathrm{EB}$ \\
\hline & & & & S-107 & EB \\
\hline \multirow[t]{2}{*}{$46^{*}$} & BX-105 & $\mathrm{CW}$, cement & & & \\
\hline & & & 148 & SX-113 & dia-earth \\
\hline \multirow[t]{3}{*}{$205 *$} & TY-103 & $1 \mathrm{C}-\mathrm{F}$ & & U-104 & dia-earth \\
\hline & TY-104 & $1 \mathrm{C}-\mathrm{F}$ & & & \\
\hline & & & 2 & U-204 & $2 \mathrm{C}$ \\
\hline \multirow[t]{5}{*}{$460 *$} & C-109(F) & $1 \mathrm{C}$ & kgal & Miscellaneous & Waste Type \\
\hline & $\mathrm{C}-112(\mathrm{~F})$ & $1 \mathrm{C}$ & $\overline{403}$ & A-102 & DSSF \\
\hline & $\mathrm{C}-108(\mathrm{~F})$ & $1 \mathrm{C}$ & & A-103 & DSSF \\
\hline & $\mathrm{C}-111(\mathrm{~F})$ & $1 \mathrm{C}$ & & & \\
\hline & $\mathrm{T}-107(\mathrm{~F})$ & $1 \mathrm{C}$ & 125 & A-106 & CC,NC,EB,B-Plant \\
\hline \multirow[t]{3}{*}{443} & BY-108(F) & EB & 7 & AX-104 & Evap, NC, Purex \\
\hline & $\mathrm{T}-109$ & EB & 45 & $\mathrm{U}-112$ & Unknown \\
\hline & TX-103 & EB & & & \\
\hline 15 & $C-105$ & PSS & & & \\
\hline
\end{tabular}




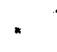

. 


\section{Enhanced Sludge Washing Spreadsheet Evaluation}

Data Summaries for 5 Core Composites 
B-110 Core 1

\begin{tabular}{|lr|}
\hline Density (kg/L) & 1.36 \\
Volume (L)/tank & 927325 \\
Mass (kg)/tank & 1261162 \\
Solids (mg/kg) & 408000 \\
Water (mg/kg) & 592000 \\
\hline
\end{tabular}

Density (kg/L)

Mass (kg)/tank

Solids (mg/kg)

W UNTREATED -

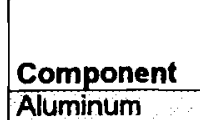

Component

Barium

Bismuth

Boron

Cadmium

Calcium

Chromium

Cobalt

Copper

Iron

Lead

Magnesium

Manganese

Nickel

Phosphate

Potassium

Silicon

Silver

Sodium

Strontium

Zinc

Zirconium

Uranium

Nitrate

Chloride

Fluoride

Carbonate

Organic Salts

Nitrite

Sulfate

Water

Radionucildes

Pu-239,240

C-14

si-90

Tc-99

Am-241

Co-60

Cs-137

1-129

(element) (element)

1158.000

19.500

1460

<DL

$<\mathrm{DL}$

869.000

824.000

$<D L$

70.000

19430.000

1130.000

203.000

102.000

$\angle D L$

50760.000

$<D L$
9420.000

26.000

97950.000

208.000

1370.000

$<D L$

161500.000

$\geq 1020.000$

$\geq 1650.000$

NR

25

$\geq 6000.000$

1030.000

$\because 9860.000$

10500.000

24504

1425

256

123531

1728

2081

12435

$592000.000 \quad 746608$

$\geq 0.024 \quad 30.268$

217273672.154

$0.135 \quad 170.257$

NR

$14.6 \quad 18412.965$

0.000022

0.028
$0.1 \mathrm{M}$ NaOH Wash

Volume wash (L) hank

(4 volume wash: 1 volume sludge)

Mass removed $(\mathrm{kg})$ )tank

3709300

932249

M NaOH Leach

\begin{tabular}{lr} 
Volume leachate (L)/ank & 396753 \\
Mass removed (kg)/ank & 63328 \\
Vol leachate (L)/kg sludge (after wash) & 1.21 \\
\hline
\end{tabular}

Residual Solids

Residual solids^ank (kg)

271289

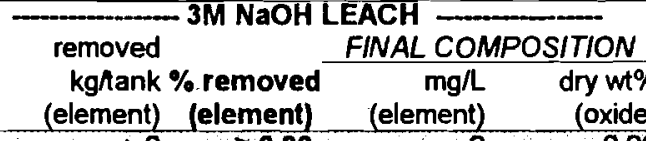

removed FINAL COMPOSITION

$\begin{array}{crr}\mathrm{kghank} \% \text { removed } & \begin{array}{r}\mathrm{mg} / \mathrm{L} \\ \text { (element) }\end{array} & \begin{array}{r}\mathrm{d} r y \mathrm{wt} \% \\ \text { (element) }\end{array} \\ \text { (element) }\end{array}$

(element)

$\geq 0 \quad \geq 0$

$1096 \quad 100 \quad 9.09$

$1039 \quad 82 \quad 7.89$

$88 \quad 45 \quad 51.43$

$64017 \quad 28621$

11880
33 $\quad 491$

109205

4.13
80

$27630 \quad 29.79$

$\geq 0$
0.58

$2 \quad 0.00$

wt\%

- RESIDUAL SOLIDS

$\mathrm{kg} / \mathrm{Ank}$
$\mathrm{mg} / \mathrm{kg}$ res
(oxide)

$:$

$0 \geq 0$

$2081 \leq 100.00$

$47798 \quad 51.54$

$302 \quad 0.33$

$\begin{array}{rr}488 & 0.53 \\ 1480 & 160\end{array}$

$\begin{array}{lr}1480 \\ 1776 & 1.60 \\ 3 & 1.91\end{array}$

$7567 \quad 7567 \leq 100.00$

$1299 \quad 1299 \quad \leq 100.00$

$12435 \leq 100.00$

305

0.33

3.15 551891

$\mathbf{7 3 . 9 2}$

3108

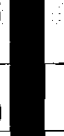

37490

Cinank

0.92

$30.268 \leqslant 100.00$

$277456 \quad 0.10$

\begin{tabular}{|r|r|}
6.306 & 3.70 \\
NR & \\
10618.984 & 57.67 \\
0.001 & 4.50 \\
\hline
\end{tabular}

7194

$63043 \quad 58.82$

194
100.00
1

$\begin{array}{rrr}1460 & 10169 & 2.42 \\ 25 & 101 & 0.02 \\ 22222 & 91331 & 21.72\end{array}$

$\begin{array}{lll}\geq 0 & & \\ 90 & 5138 & 1.22\end{array}$

$\begin{array}{llllll}33184 & 51.84 & 76418 & 39.51\end{array}$

$63043 \quad 58.82$

33
14326
262
1718
$\geq 0$
$\geq 0$
$\geq 0$
$\geq 0$
$\geq 0$
$\geq 0$
$\geq 0$
$\geq 0$
$\geq 0$
$\geq 0$
$\geq 0$

1290

$\begin{array}{rrr}20 & 198 & 0.05 \\ 43 & 198 & 30.47 \\ 24301 & 128095 & 1.35 \\ 1425 & 5658 & 1.35\end{array}$

$\begin{array}{rrr}243 & 1488 & 0.35\end{array}$

$\begin{array}{rrr}129 & 750 & 0.18 \\ 20 & & \\ 2212 & 6090 & 1.45\end{array}$

89802

$130 \quad 21.36$

$71182 \quad 16.93$

$\begin{array}{ll}1144 & 0.27\end{array}$

$\begin{array}{rr}7883 & 1.87 \\ \geq 0 & \geq 0.00\end{array}$

$\begin{array}{ll}\geq 0 & \geq 0.00 \\ \geq 0 & \geq 0.00\end{array}$

$\geq 0 \quad \geq 0.00$

$\geq 0 \quad \geq 0.00$

$\geq 0 \quad \geq 0.00$

$\begin{array}{ll}\geq 0 & \geq 0.00 \\ >0 & >0.00\end{array}$

$\geq 0 \quad \geq 0.00$

$\geq 0 \quad \geq 0.00$

1000000

100.00

1


C-109 Core 47

Density (kg/L)

Volume (L)hank

Mass (kg)/ank

Solids (mg/kg)

Water $(\mathrm{mg} / \mathrm{kg})$

$\begin{array}{r}1.2 \\ 234670 \\ 281604 \\ 785000 \\ 215000 \\ \hline\end{array}$

$0.1 \mathrm{M} \mathrm{NaOH}$ Wash

Volume wash (L) hank

938680

volume sludge)

Mass removed (kg)/tank
$3 \mathrm{M} \mathrm{NaOH} \mathrm{Leach}$

75934

\begin{tabular}{|lr|}
\hline Volume leachate (L)/ank & 520655 \\
Mass removed $(\mathrm{kg})$ /ank & 86221 \\
Vol leachate $(\mathrm{L}) / \mathrm{kg}$ sludge (after wash) & 2.53 \\
\hline
\end{tabular}

Residual Sollds

Residual solids/tank (kg) 108413

Vol leachate $(\mathrm{L}) / \mathrm{kg}$ sludge (after wash) $\quad 2.53$

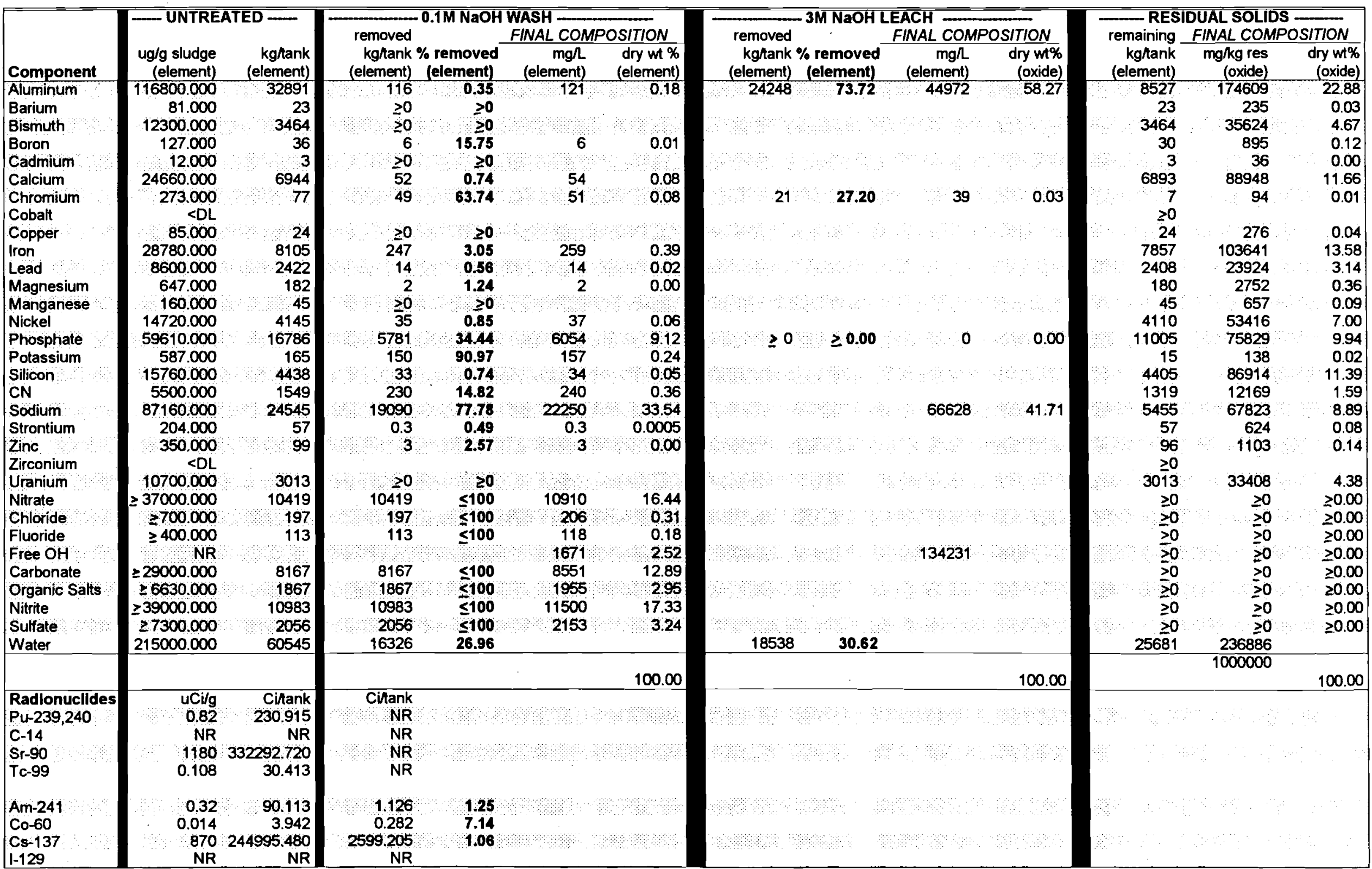


C-112 Core 36

Density (kg/L)

Volume (L)Aank

Mass $(\mathrm{kg})$ /tank

Solids ( $\mathrm{mg} / \mathrm{kg}$ )

Water ( $\mathrm{mg} / \mathrm{kg})$
$0.1 \mathrm{M}$ NaOH Wash

Volume wash (L)hank

(4 volume wash: 1 volume sludge)

Mass removed (kg)hank

1574560

393640

551096

550000

450000
$3 \mathrm{M} \mathrm{NaOH}$ Leach

\begin{tabular}{|lr|}
\hline Volume leachate (L)hank & 397745 \\
Mass removed (kg)/hank & 30631 \\
Vol leachate (L)/kg sludge (after wash) & 1.83 \\
\hline
\end{tabular}
Residual Sollds

Residual solids/tank (kg) 163668$$
\text { L_- - — - - }
$$

$0.1 \mathrm{M} \mathrm{NaOH} \mathrm{WASH}$

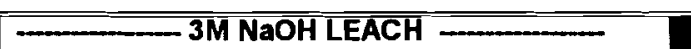

(2)

RESIDUAL SOLIDS \begin{tabular}{rr}
$\begin{array}{r}\text { ug/g sludge } \\
\text { (element) }\end{array}$ & $\begin{array}{r}\mathrm{kg} / \text { tank } \\
\text { (element) }\end{array}$ \\
\hline 6400.000 & 3527 \\
76.800 & 42
\end{tabular} kg/tank \% removed $\frac{\text { FINAL COMPOSITION }}{\mathrm{mg} / \mathrm{dry} w \%}$ (element) (element) (element) (element)

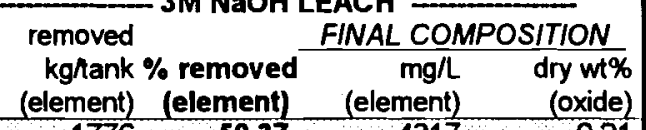

Component Aluminum Barium

Bismuth

Boron

Cadmium

Calcium

Chromium

Cobalt

Copper

Iron

Lead

Magnesium

Manganese

Nickel

Phosphate

Polassium

Silicon

CN

Sodium

Strontium

Zinc

Zirconium

Uranium

Nitrate

Chloride

Fluoride

Free OH

Carbonate

Organic Salts

Nitrite

Sulfate

Water

\begin{tabular}{|l} 
\\
\hline Radionuclldes
\end{tabular}

Put239,240

C-14

Sr-90

Tc-99

Am-241

Co-60

Cs-137

i-129

\begin{tabular}{|c|c|}
\hline 76.800 & 42 \\
\hline NR & NR \\
\hline 143.000 & 79 \\
\hline 23.200 & 13 \\
\hline 20390.000 & 11237 \\
\hline 182.000 & 100 \\
\hline$<D L$ & \\
\hline 50.100 & 28 \\
\hline 26010.000 & 14334 \\
\hline 1050.000 & 579 \\
\hline 610.000 & 336 \\
\hline 266.000 & 147 \\
\hline 10570.000 & 5825 \\
\hline 110280.000 & 60775 \\
\hline 645.000 & 355 \\
\hline 1930.000 & 1064 \\
\hline 7100.000 & 3913 \\
\hline 120700.000 & 66517 \\
\hline 396.000 & 218 \\
\hline 370,000 & 204 \\
\hline 33.600 & 19 \\
\hline 10490.000 & 5781 \\
\hline$\geq 71500.000$ & 39403 \\
\hline$>1050.000$ & 579 \\
\hline$\geq 450.000$ & 248 \\
\hline NR & NR \\
\hline$\geq 19750.000$ & 10884 \\
\hline$\geq 7590.000$ & 4183 \\
\hline$>52500.000$ & 28933 \\
\hline 13700.000 & 7550 \\
\hline 450000.000 & 247993 \\
\hline $\mathrm{uCi} / \mathrm{g}$ & Citank \\
\hline 0.059 & 32515 \\
\hline 0.004 & 2.204 \\
\hline 507 & 279405.672 \\
\hline 0.107 & 58.967 \\
\hline 0.061 & 33.617 \\
\hline 0.006 & 3.307 \\
\hline 792 & 436468.032 \\
\hline NR & 10 \\
\hline
\end{tabular}

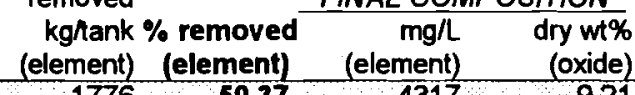

177

$\begin{array}{lrrr}449 & 12.72 & \text { (element) } & \text { (element) } \\ \geq 0 & \geq 0 & 0.240\end{array}$

$16 \quad 20.77$

16
$\geq 0$
212

$\begin{array}{rrrr}212 & 1.89 & 123 & 0.11\end{array}$

$\begin{array}{llll}61 & 60.99 & 35 & 0.03\end{array}$

$\geq 0$
768

$768 \quad 5.36$

$\frac{20}{16}$

2
436

7.49
43.81

77.98

3.62

18.52

89.77

725

9715

1.52
1.57

1.57
$>0$

$\geq 0$

2533

39403

$\leq 100$
$\leq 100$

579
248

$\leq 100$
$\leq 100$

$10884 \leq 100$

4183

$28933<100$

$7550 \quad \leq 100$

150266

60.59

100.00

13784

Cifank
NR
NR
NR
NR
NR $\quad$
NR
NR
NR




U-110 Core 14
\begin{tabular}{|lr|}
\hline Density (kg/L) & 1.5 \\
Volume (L)/tank & 704010 \\
Mass (kg)/tank & 1056015 \\
Solids $(\mathrm{mg} / \mathrm{kg})$ & 739500 \\
Water $(\mathrm{mg} / \mathrm{kg})$ & 260500 \\
\hline
\end{tabular}

$0.1 M \mathrm{NaOH}$ Wash

Volume wash (L)/tank

( 4 volume wash: 1 volume sludge)

Mass removed (kg)hank

2816040

289858

$3 \mathrm{M} \mathrm{NaOH}$ Leach

\begin{tabular}{|lr|}
\hline Volume leachate $(\mathrm{L})$ /tank & 2145942 \\
Mass removed $(\mathrm{kg})$ /tank & 527187 \\
Vol leachate $(\mathrm{L}) \mathrm{kg}$ sludge (after wash) & 2.80 \\
\hline
\end{tabular}

ResIdual Sollds

Residual solidstank (kg) 253724

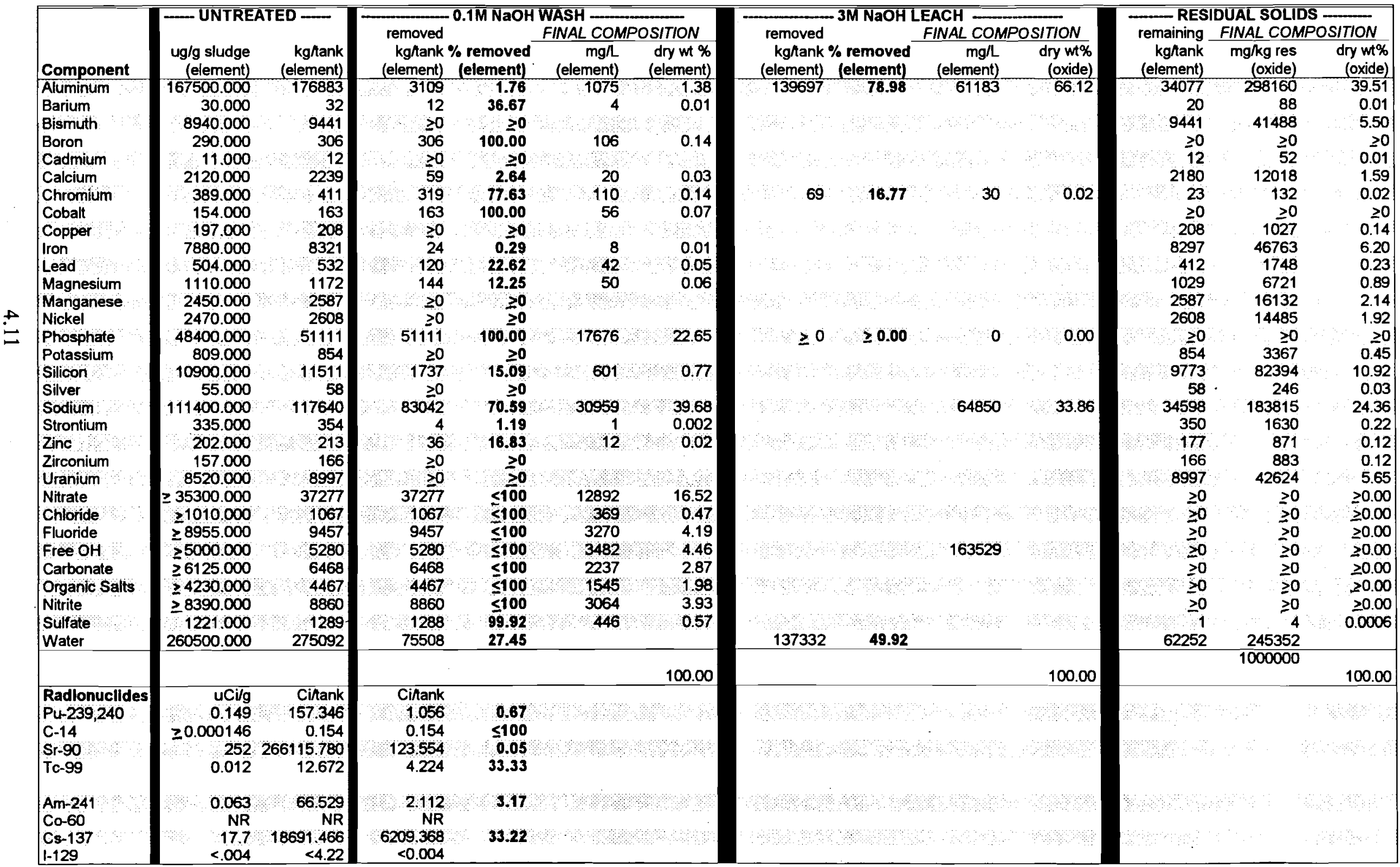


B-201 Core 27

Density (kg/L) Volume (L)hank Mass (kg)/tank Solids (mg/kg) Water (mg/kg)
$0.1 \mathrm{M}$ NaOH Wash

Volume wash (L)/tank

(4 volume wash: 1 volume sludge)

Mass removed $(\mathrm{kg})$ )tank

423920

105980

132475

627000
$3 \mathrm{M} \mathrm{NaOH} \mathrm{Leach}$

37376
ResIdual Soilds

Residual solids/tank (kg) $\quad 84580$

Volume leachate (L)hank $\quad 21769$

Mass removed $(\mathrm{kg})$ /ank $\quad 1850$

Vol leachate (L)/kg sludge (after wash) $\quad 0.23$

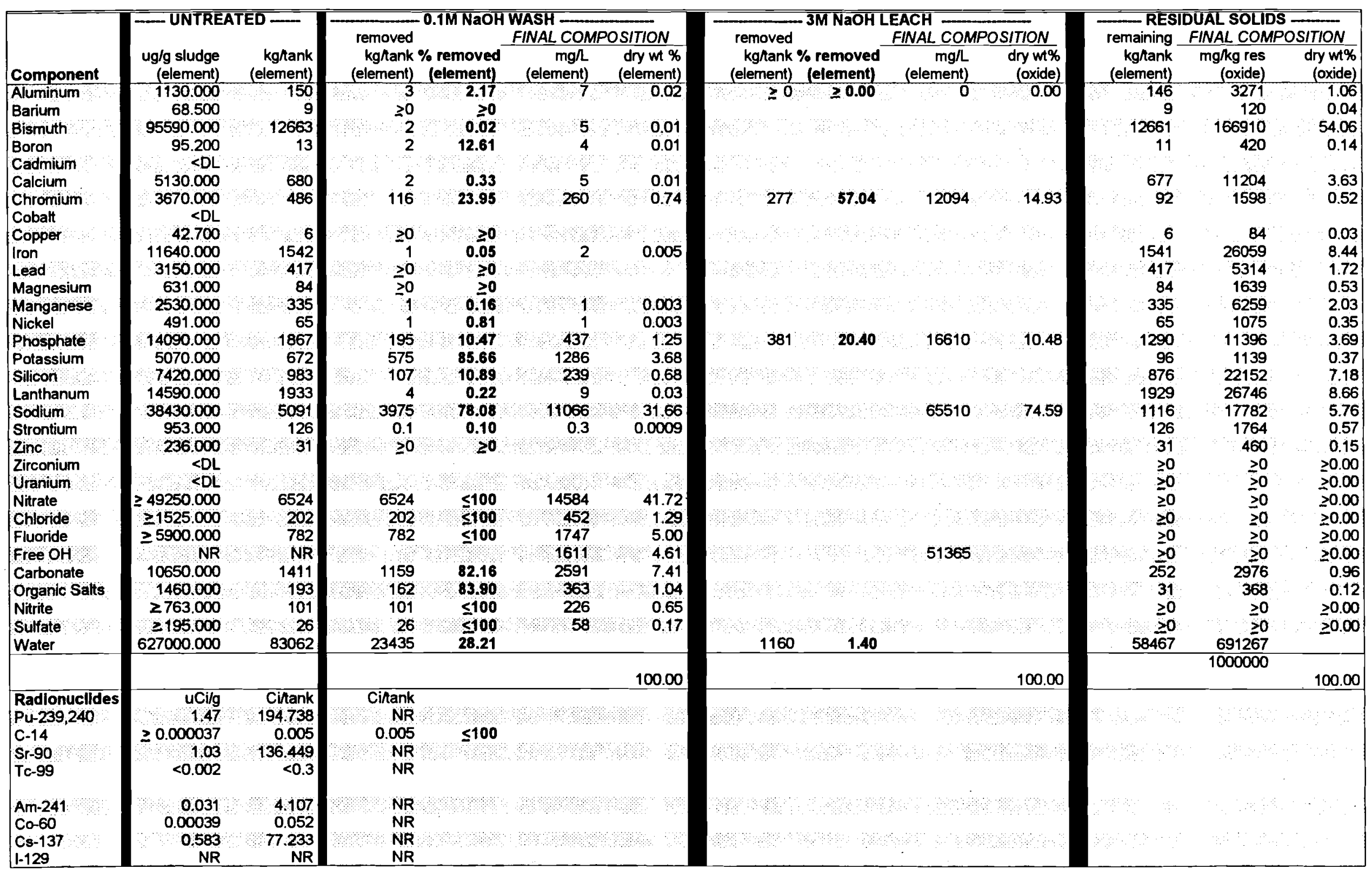


Enhanced Sludge Washing Spreadsheet Evaluation

Data Summaries for 20 Specific Tank Sludges 
B-110

\begin{tabular}{|lr|}
\hline Density (kg/L) & 1.35 \\
Volume (L)/tank & 927325 \\
Mass (kg)/tank & 1251889 \\
Solids (mg/kg) & 430000 \\
Water (mg/kg) & 570000 \\
\hline
\end{tabular}

$0.1 \mathrm{M}$ NaOH Wash

Volume wash (L)tank

(4 volume wash:1 volume sludge)

Mass removed (kg)/ank
$3 \mathrm{M} \mathrm{NaOH}$ Leach

709300

952011
Volume leachate (L) rank

Mass removed $(\mathrm{kg})$ /tank

Vol leachate $(L) / k g$ sludge (after wash)
Residual Sollids

Residual solids/tank (kg)

250074

1214

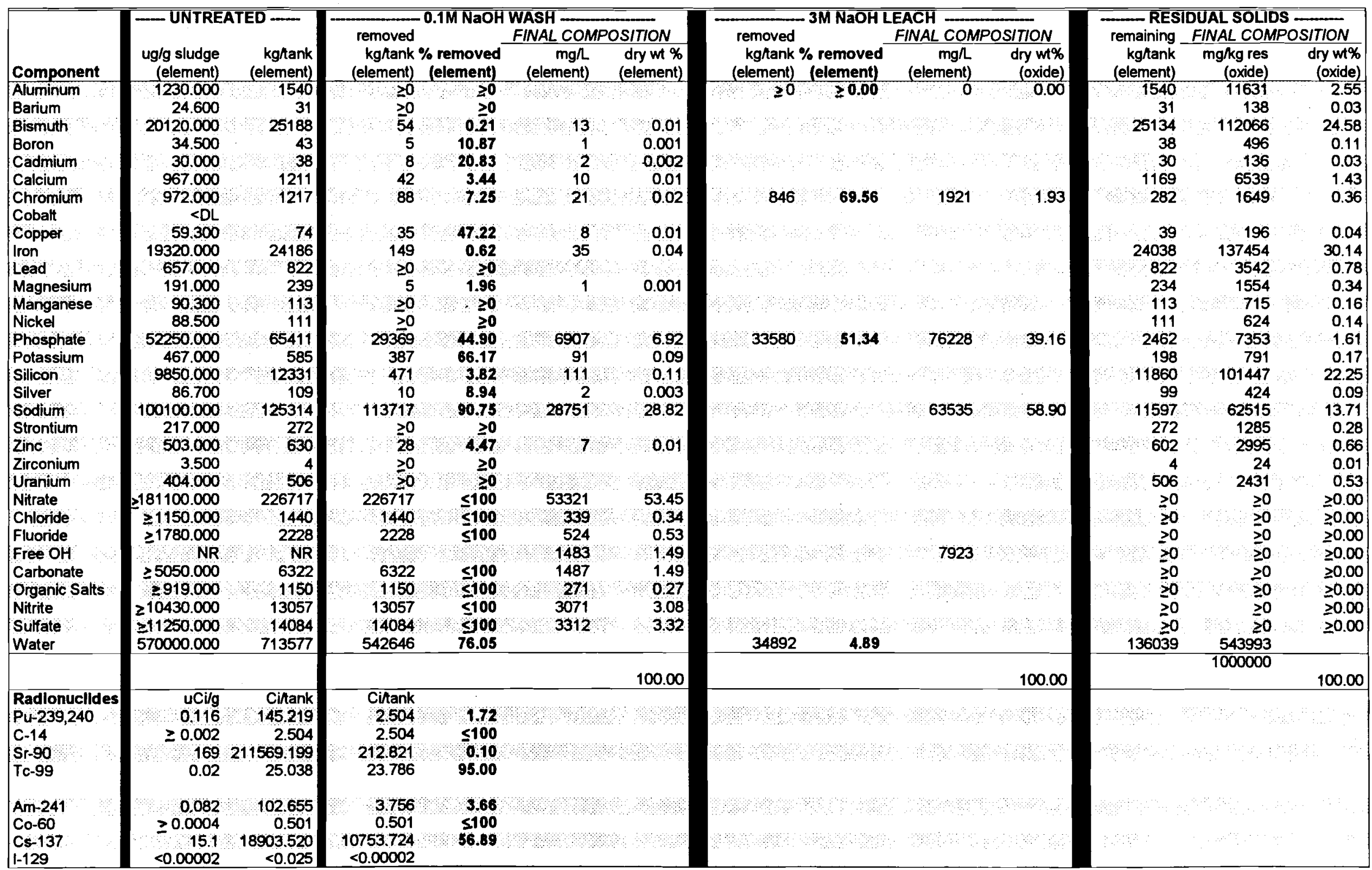




\begin{tabular}{|lr|}
\hline B-111 \\
\hline Density (kg/) & 1.25 \\
Volume (L) Nank & 893260 \\
Mass (kg)/ank & 1116575 \\
Solids (mg/kg) & 363000 \\
Water (mg/kg) & 637000 \\
\hline
\end{tabular}

$0.1 \mathrm{M} \mathrm{NaOH}$ Wash

Volume wash (L)/tank

(4 volume wash:1 volume sludge)

Mass removed (kg)/tank

3573040

3573040

834993

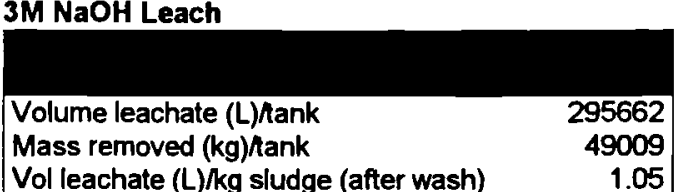

Residual Sollds

Residual solids/tank (kg)

243251

Vol leachate (L)/kg sludge (after wash) $\quad 1.05$

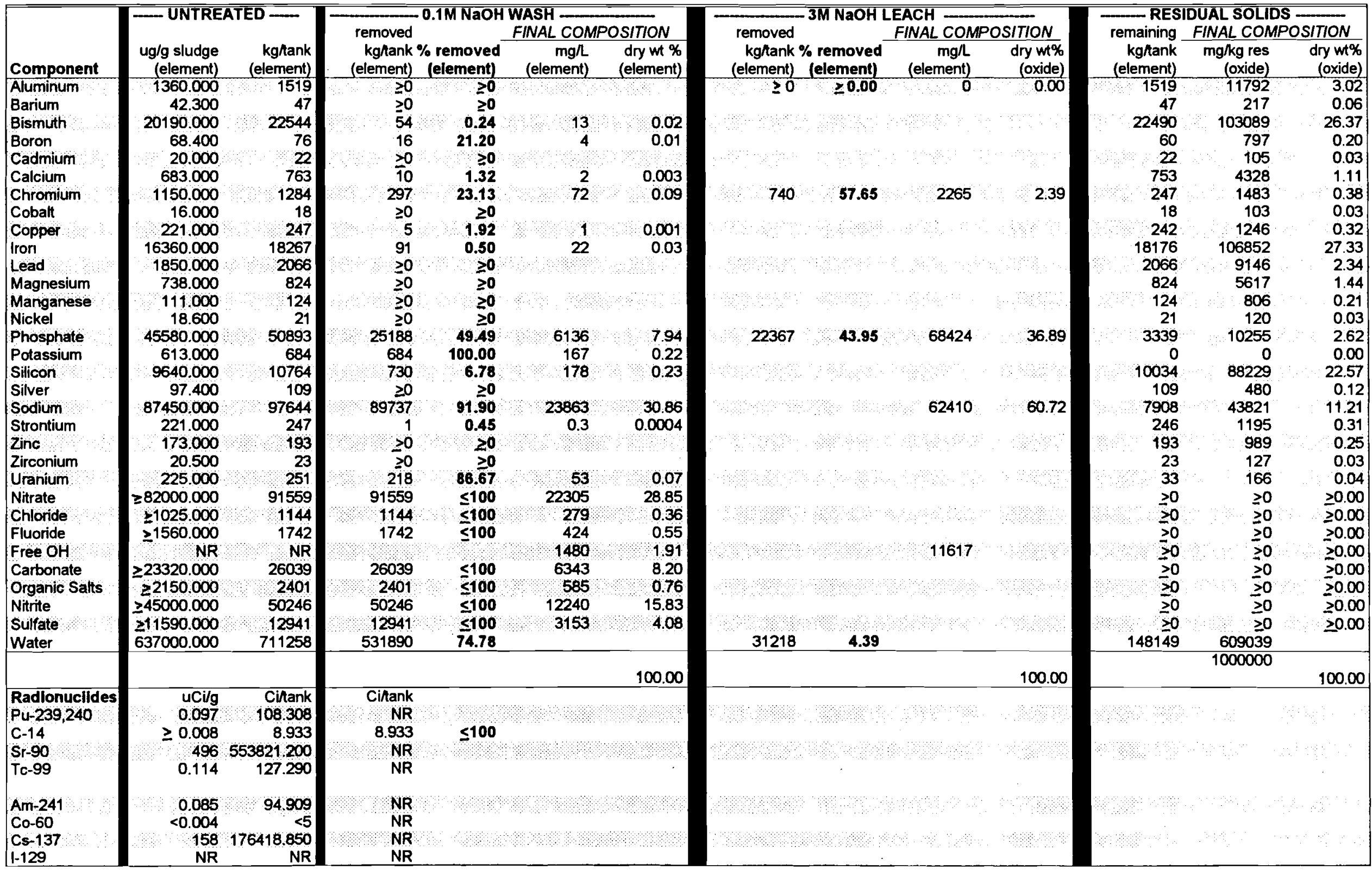


B-201

Density (kg/L)

Volume (L)Aank $\quad 1.25$

Mass (kg)/ank $\quad 132475$

Solids (mg/kg) $\quad 396000$

Water $(\mathrm{mg} / \mathrm{kg}) \quad 604000$
$0.1 \mathrm{M}$ NaOH Wash

Volume wash (L) Mank

(4 volume wash:1 volume sludge)

Mass removed (kg)hank
$3 \mathrm{M} \mathrm{NaOH}$ Leach

36736

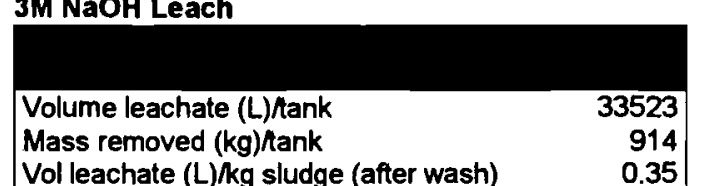

Residual Solids

Residual solids/ank (kg)

101118

Vol leachate (L) $/ \mathrm{kg}$ sludge (after wash)

0.35

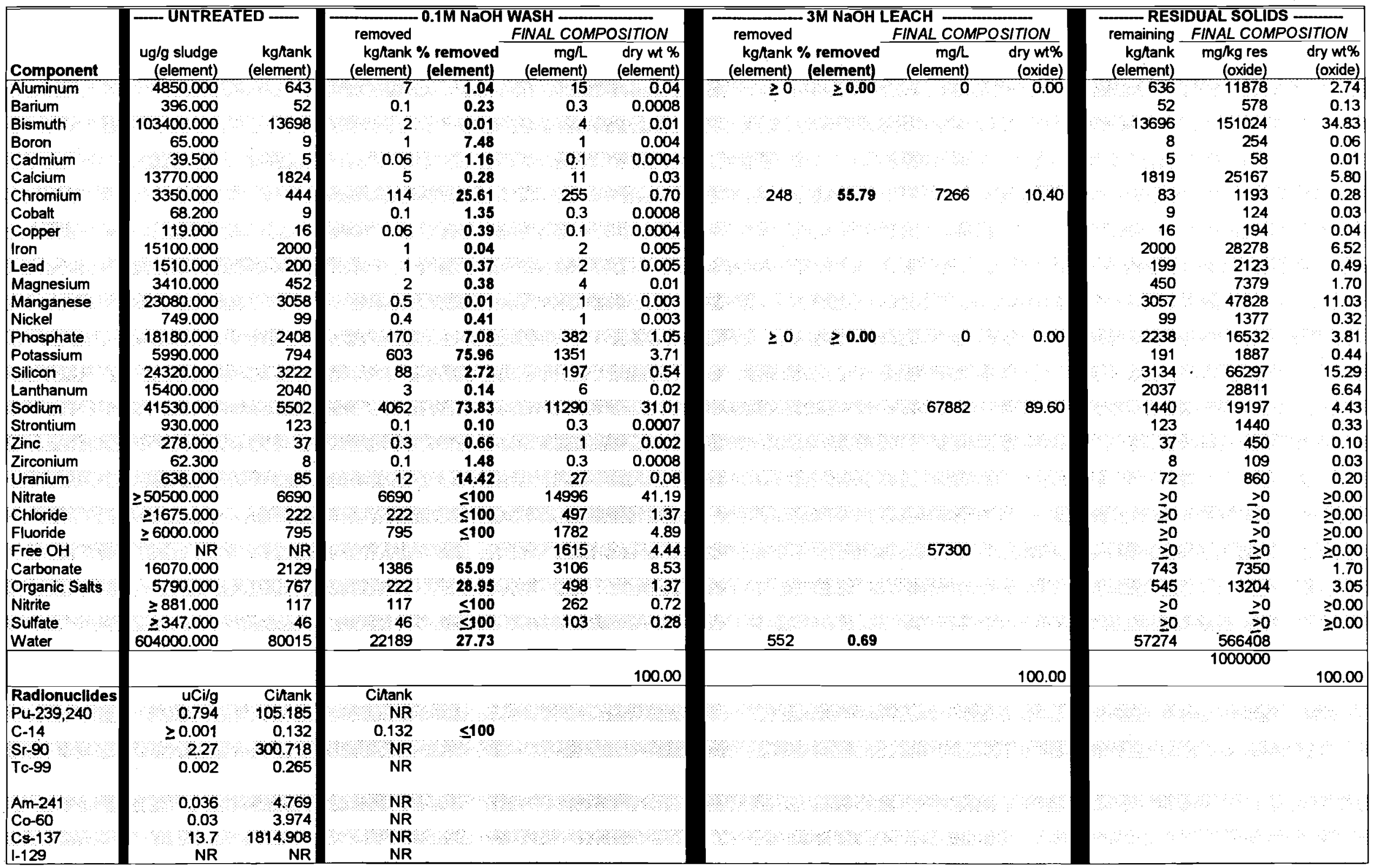




\section{BX-105}

\begin{tabular}{|lr|}
\hline Density (kg/L) & 1.69 \\
Volume (L)/tank & 174110 \\
Mass (kg)/tank & 294246 \\
Solids (mg/kg) & 430000 \\
Water (mg/kg) & 570000 \\
\hline
\end{tabular}

Water $(\mathrm{mg} / \mathrm{kg}) \quad \mathbf{5 7 0 0 0 0}$
$0.1 \mathrm{M}$ NaOH Wash

Volume wash (L)/tank

(4 volume wash:1 volume sludge)

Mass removed $(\mathrm{kg})$ hank

696440

696440

118006
$3 \mathrm{M} \mathrm{NaOH}$ Leach

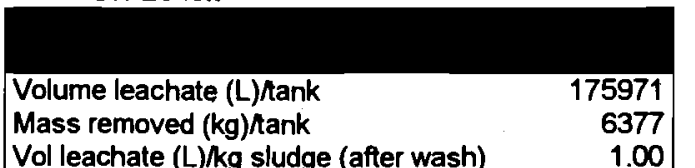

Residual Sollds

Residual solids/tank (kg) 167500

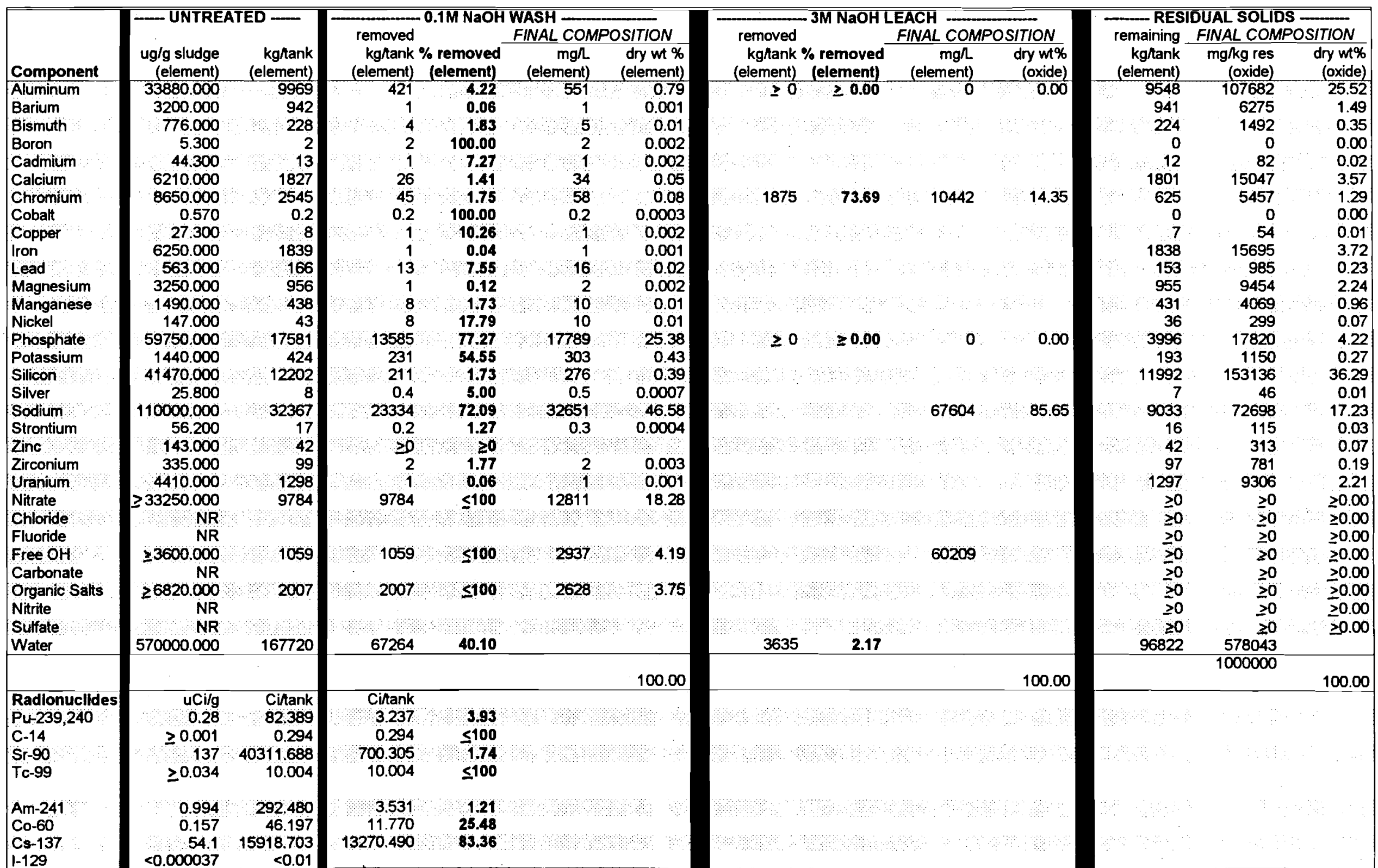




\section{BX-107}

Density $(\mathrm{kg} / \mathrm{L})$

Volume (L)hank $\quad 1302040$

Mass (kg)/tank 1562448

Solids (mg/kg)

432600

$0.1 \mathrm{M}$ NaOH Wash

Volume wash (L)/tank
(4 volume wash: 1 volume sludge)

Mass removed $(\mathrm{kg})$ )tank
$3 \mathrm{M} \mathrm{NaOH}$ Leach

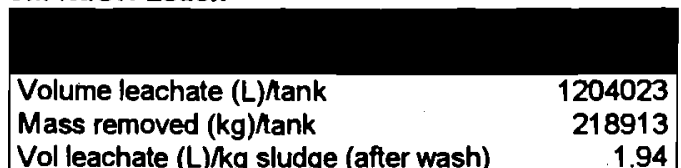

Residual Sollds

Residual solids/tank (kg)

416897

942948

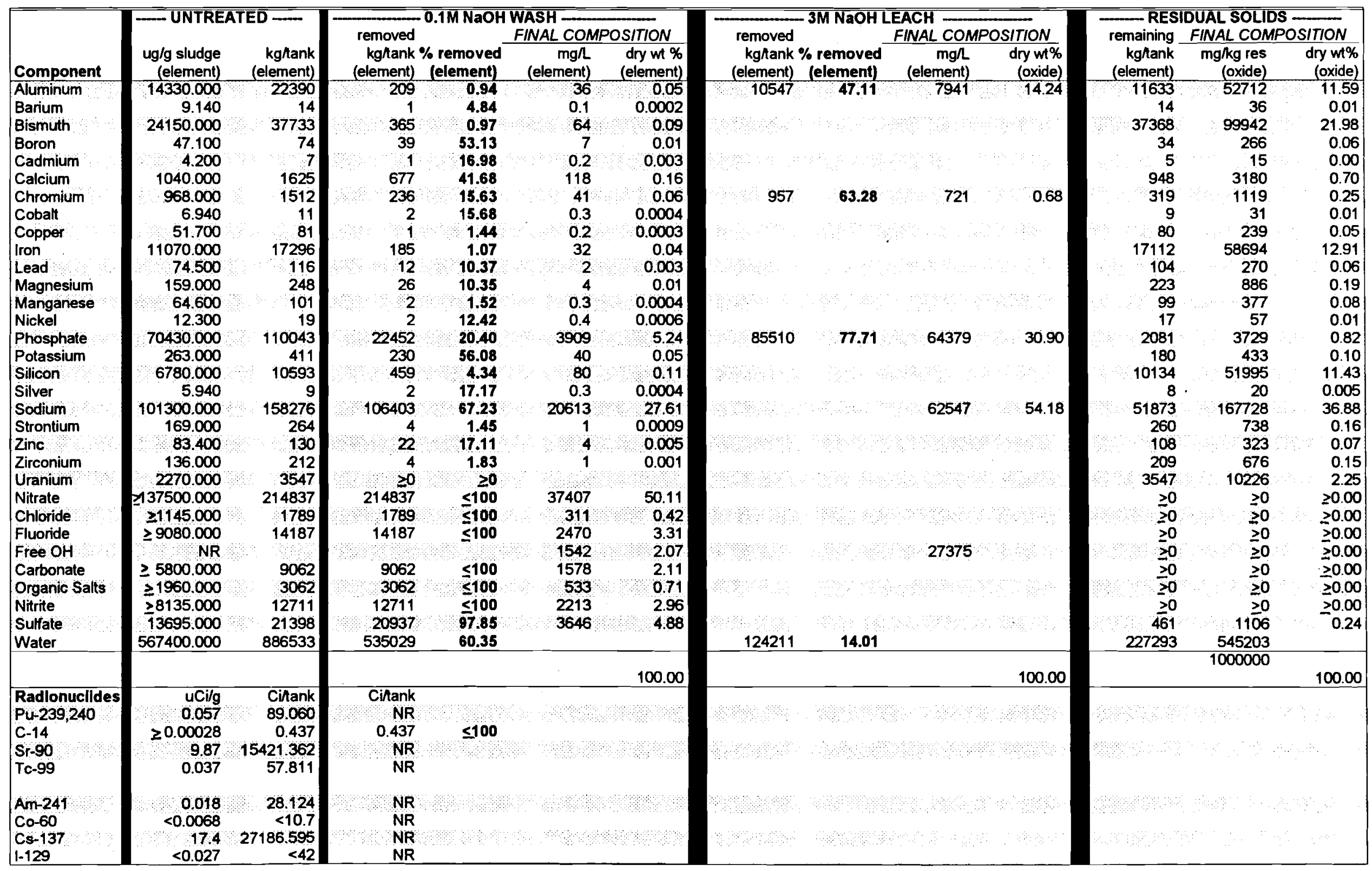




\section{C.103}

\begin{tabular}{|lr|}
\hline Density (kg/L) & 1.34 \\
Volume (L)/tank & 234670 \\
Mass (kg)/tank & 314458 \\
Solids (mg/kg) & 389000 \\
Water (mg/kg) & 611000 \\
\hline
\end{tabular}

$0.1 \mathrm{M} \mathrm{NaOH}$ Wash

Volume wash (L)hank

(4 volume wash:1 volume sludge)

Mass removed (kg)/ank
3M NaOH Leach

938680

18124

Volume leachate (L)Aank 91404

Mass removed $(\mathrm{kg})$ /tank $\quad 532$

Vol leachate (L)/kg sludge (after wash) $\quad 0.31$

Resldual Sollds

Residual solidstank (kg) $\quad 317302$

(1)

RESIDUAL SOLIDS -

\begin{tabular}{|c|c|c|c|}
\hline 0. & $\overline{\mathbf{A S H}}$ & $-3 \mathrm{~N}$ & $\overline{\overline{\mathbf{C H}}}$ \\
\hline removed & FINAL COMPOSITION & removed & FINAL COMPOSITION \\
\hline $\begin{array}{c}\text { kgAtank \% removed } \\
\text { (element) (element) }\end{array}$ & $\begin{array}{r}\mathrm{mg} / \mathrm{L} \\
\text { (element) }\end{array}$ & $\begin{array}{l}\text { kg/tank \% removed } \\
\text { (element) (element) }\end{array}$ & $\begin{array}{r}\mathrm{mg} / \mathrm{L} \\
\text { (element) }\end{array}$ \\
\hline
\end{tabular}

Component

Aluminum

Barium

Bismuth

Boron

Cadmium

Calcium

Chromium

Cobalt

Copper

Iron

Magnesium

Manganese

Nickel

Phosphate.

Potassium

Silicon

Silver

Sodium

Strontium

Zinc

Zirconium

Uranium

Nitrate

Chloride

Fluoride

Free $\mathrm{OH}$

Carbonate

Organic Salts

Nitrite

Sulfate

Water

Radionuclldes

Pu-239,240

C-14

Sr-90

Tc-99

Am-241

Co-60

Cs-137

I-129

\begin{tabular}{|c|c|c|c|c|c|}
\hline \multirow{2}{*}{\multicolumn{2}{|c|}{ - UNTREATED — }} & \multirow{2}{*}{\multicolumn{4}{|c|}{$0.1 \bar{M} \mathrm{NaOH}$ WASH }} \\
\hline & & & & & FINAL COMPOSITION \\
\hline $\begin{array}{r}\text { ug/g sludge } \\
\text { (element) }\end{array}$ & $\begin{array}{r}\text { kg/tank } \\
\text { (element) }\end{array}$ & \multicolumn{2}{|c|}{$\begin{array}{l}\text { removed } \\
\text { kgAank \% removed } \\
\text { (element) (element) }\end{array}$} & $\begin{array}{r}\mathrm{mg} / \mathrm{l} \\
\text { (element) }\end{array}$ & $\begin{array}{l}\text { dry wt \% } \\
\text { (element) }\end{array}$ \\
\hline 14510.000 & 4563 & $\frac{2}{3}$ & 0.06 & 133 & 0.02 \\
\hline 4960.000 & 1560 & 1 & 0.04 & 1 & 0.01 \\
\hline 722.000 & 227 & 21 & 9.26 & 22 & 0.19 \\
\hline 2.400 & 1 & $\geq 0$ & $\geq 0$ & & \\
\hline 509.000 & 160 & 2 & 1.36 & 2 & 0.02 \\
\hline 11110.000 & 3494 & 12 & 0.34 & 12 & 0.11 \\
\hline 618.000 & 194 & 6 & 2.85 & 6 & 0.05 \\
\hline 63.800 & 20 & 11 & 56.90 & 12 & 0.11 \\
\hline 855.000 & 269 & 5 & 1.91 & 5 & 0.05 \\
\hline 86660.000 & 27251 & 2 & 0.01 & 2 & 0.02 \\
\hline 3560.000 & 1119 & 8 & 0.73 & 9 & 0.08 \\
\hline 6190.000 & 1946 & 3 & 0.15 & 3 & 0.03 \\
\hline 2480.000 & 780 & 4 & 0.48 & 4 & 0.03 \\
\hline 2850.000 & 896 & 14 & 1.58 & 15 & 0.13 \\
\hline 12530.000 & 3940 & 720 & 18.28 & 758 & 6.66 \\
\hline 1400.000 & 440 & 54 & 12.21 & 57 & 0.50 \\
\hline 70620.000 & 22207 & 29 & 0.13 & 30 & 0.27 \\
\hline 222.000 & 70 & 4 & 5.99 & 4 & 0.04 \\
\hline 50500.000 & 15880 & 2299 & 14.48 & 4693 & 41.26 \\
\hline 117.000 & 37 & $\geq 0$ & $\geq 0$ & & \\
\hline 175.000 & 55 & $\geq 0$ & $\geq 0$ & & \\
\hline 12210.000 & 3840 & 12 & 0.30 & 12 & 0.11 \\
\hline 3440.000 & 1082 & 442 & 40.89 & 466 & 4.09 \\
\hline$\geq 2810.000$ & 884 & 884 & $\leq 100$ & 930 & 8.18 \\
\hline NR & & & & & \\
\hline NR & & & & & \\
\hline NR & & & & 1680 & 14.77 \\
\hline NR & & & & & \\
\hline$\geq 8000.000$ & 2516 & 2516 & 5100 & 2649 & 23.28 \\
\hline NR & & & & & \\
\hline NR & & & & & \\
\hline 611000.000 & 192134 & 11074 & 5.76 & & \\
\hline & & & & & 100.00 \\
\hline $\mathrm{uCi} / \mathrm{g}$ & Cihank & Citank & & & \\
\hline 136 & 4276.626 & 2.201 & 0.05 & & \\
\hline$\geq 0$ & $\geq 0$ & & & & \\
\hline 2710 & 852180.638 & 326722 & 0.04 & & \\
\hline 0.264 & 83.017 & 5.346 & 6.44 & & \\
\hline 1.35 & 424.518 & 2.516 & 0.59 & & \\
\hline 3.95 & 1242.108 & 24.842 & 2.00 & & \\
\hline 59.4 & 18678.793 & 7672770 & 41.08 & & \\
\hline$\geq 0.000011$ & 0.003 & 0.003 & $\leq 100.00$ & & \\
\hline
\end{tabular}

remaining FINAL COMPOSITION

kgtank $\mathrm{mg} / \mathrm{kg}$ res dry wt\%

\begin{tabular}{rrr} 
(element) & (oxide) & (oxide) \\
\hline 4560 & 27148 & 631
\end{tabular}

$\begin{array}{rrr}4560 & 27148 & 6.31 \\ 1559 & 5489 & 1.28\end{array}$

$\begin{array}{rrr}206 & 724 & 0.17 \\ 1 & 8 & 0.00\end{array}$

$\begin{array}{rrrr}158 & 568 & 0.00 \\ 3482 & 15351 & & 3.57\end{array}$

$\begin{array}{lll}3482 & 15351 & 3.57\end{array}$

$\begin{array}{llll}142 & 72.86 & 1544 & 2.38\end{array}$

$68755 \quad 97.62$

$\begin{array}{rrr}47 & 217 & 0.05 \\ 9 & 38 & 0.01 \\ 264 & 1041 & 0.24\end{array}$

$27249 \quad 122803 \quad 28.53$

$\begin{array}{lll}1111 & 3772 & 0.88\end{array}$

$\begin{array}{lll}1943 & 10155 & 2.36\end{array}$

$\begin{array}{lll}776 & 3870 & 0.90 \\ 882 & 3917 & 0.91 \\ 3220 & 7581 & 1.76\end{array}$

$\begin{array}{llll}3220 & 7581 & & \\ 386 & 1218 & 1.76\end{array}$

$\begin{array}{lll}386 & 1218 & 0.28\end{array}$

$\begin{array}{lll}22178^{\circ} & 149508 & 34.74\end{array}$

$\begin{array}{rrr}66 & 222 & 0.05 \\ 13581 & 57698 & 13.41\end{array}$

$\begin{array}{rrr}13581 & 57698 & 13.41 \\ 37 & 137 & 0.03 \\ 55 & 216 & 0.05\end{array}$

$\begin{array}{rrr}55 & 216 & 0.05 \\ 3828 & 16299 & 3.79\end{array}$

$639 \quad 2422$

$\geq 0 \quad \geq 0 \quad \geq 0.00$

$\begin{array}{lll}\geq 0 & \geq 0 & \geq 0.00\end{array}$

$\begin{array}{lll}\geq 0 & \geq 0 & \geq 0.00\end{array}$

$\geq 0.00$

$\geq 0.00$

$\geq 0.00$

$\geq 0.00$

$325 \quad 0.17$

100.00

$\begin{array}{rrr}\geq 0 & \geq 0 & \geq 0.00 \\ 180735 & 569598 & \end{array}$


C-106

\begin{tabular}{|lr|}
\hline Density (kg $/$ ) & 1.43 \\
Volume (L)/tank & 745645 \\
Mass (kg)/tank & 1066272 \\
Solids (mg/kg) & 475000 \\
Water (mg/kg) & 525000 \\
\hline
\end{tabular}

$0.1 \mathrm{M}$ NaOH Wash

Volume wash (L)/tank

(4 volume wash:1 volume sludge)

Mass removed $(\mathrm{kg})$ ) tank
3M NaOH Leach

2982580

133635
Volume leachate (L)/tank $\quad 628267$

Mass removed (kg)/tank

Vol leachate (L)/kg sludge (after wash)
Resldual Sollds

Residual solidstank (kg) 972019

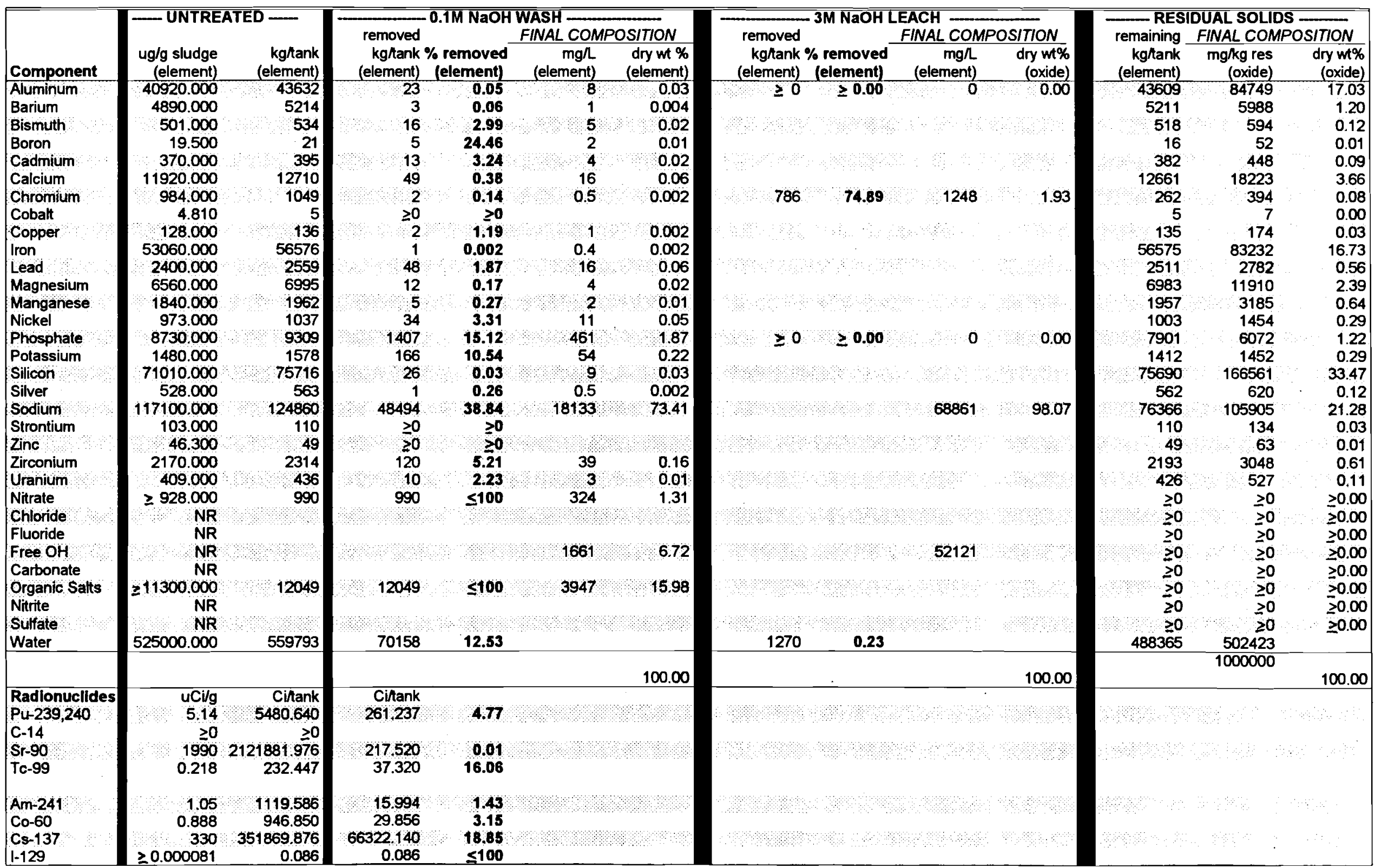




C-109
\begin{tabular}{|lr|}
\hline Density (kg/L) & 1.2 \\
Volume (L)/tank & 234670 \\
Mass (kg)/tank & 281604 \\
Solids (mg/kg) & 753500 \\
Water (mg/kg) & 246500 \\
\hline
\end{tabular}

$0.1 \mathrm{M}$ NaOH Wash

Volume wash (L)hank

(4 volume wash:1 volume sludge)

Mass removed (kg)/tank

938680
74503
$3 \mathrm{M} \mathrm{NaOH}$ Leach

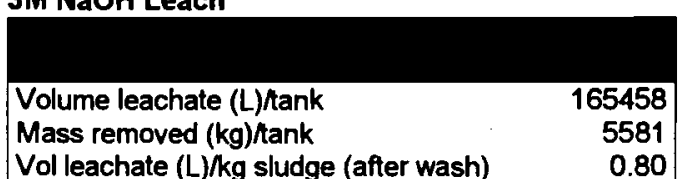

Vol leachate (L)/kg sludge (after wash) $\quad 0.80$
Residual Solids

Residual solids/tank (kg) 106466

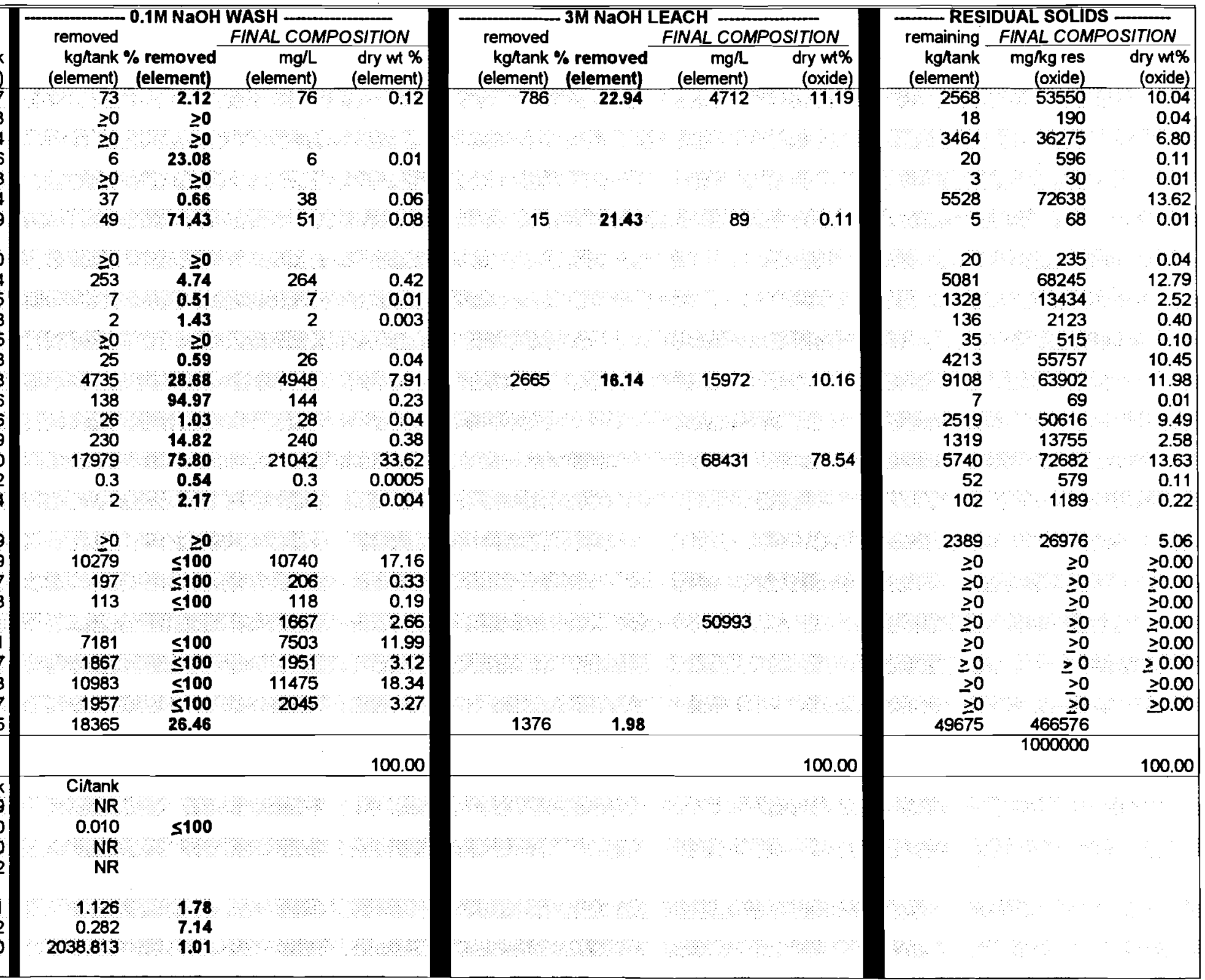

Water $(\mathrm{mg} / \mathrm{kg}) \quad 246500$

\begin{tabular}{|c|c|c|c|c|c|c|}
\hline \multirow[b]{2}{*}{ Component } & \multicolumn{2}{|c|}{-- UNTREATED - } & \multicolumn{4}{|c|}{ removed $0.1 \mathrm{M}$ NaOH WASH } \\
\hline & $\begin{array}{r}\text { ug/g sludge } \\
\text { (element) }\end{array}$ & $\begin{array}{r}\text { kg/tank } \\
\text { (element) }\end{array}$ & \multicolumn{2}{|c|}{$\begin{array}{l}\text { kghtank \% removed } \\
\text { (element) (element) }\end{array}$} & $\begin{array}{r}\mathrm{mg} / \mathrm{L} \\
\text { (element) }\end{array}$ & $\begin{array}{l}\text { dry wt \% } \\
\text { (element) }\end{array}$ \\
\hline Aluminum & 12170.000 & 3427 & 73 & 2.12 & 76 & 0.12 \\
\hline Barium & 64.300 & 18 & $\geq 0$ & $\geq 0$ & & \\
\hline Bismuth & 12300.000 & 3464 & $\geq 0$ & $>0$ & & \\
\hline Boron & 91.000 & 26 & 6 & 23.08 & 6 & 0.01 \\
\hline Cadmium & 10.000 & & $\geq 0$ & $\geq 0$ & & \\
\hline Calcium & 19760.000 & 5564 & 37 & $0 . \overline{66}$ & 38 & 0.06 \\
\hline Chromium & 245.000 & 69 & 49 & 71.43 & 51 & 0.08 \\
\hline Cobalt & $<D L$ & & & & & \\
\hline Copper: & 71,000 & 20 & $\geq 0$ & $\geq 0$ & & \\
\hline Iron & 18940.000 & 5334 & 253 & 4.74 & 264 & 0.42 \\
\hline Lead & 4740.000 & 1335 & 7 & 0.51 & 7 & 0.01 \\
\hline Magnesium & 491.000 & 138 & 2 & 1.43 & 2 & 0.003 \\
\hline Manganese & 123.000 & 35 & $\geqslant 0$ & $\geq 0$ & & \\
\hline Nickel & 15050.000 & 4238 & 25 & $0 . \overrightarrow{59}$ & 26 & 0.04 \\
\hline Phosphate & 58620.000 & 16508 & 4735 & 28.68 & 4948 & 7.91 \\
\hline Potassium & 517.000 & 146 & 138 & 94.97 & 144 & 0.23 \\
\hline Silicon & 9040.000 & 2546 & 26 & 1.03 & 28 & 0.04 \\
\hline $\mathrm{CN}$ & 5500.000 & 1549 & 230 & 14.82 & 240 & 0.38 \\
\hline Sodium & 84230.000 & 23720 & 17979 & 75.80 & 21042 & 33.62 \\
\hline Strontium & 186.000 & 52 & 0.3 & 0.54 & 0.3 & 0.0005 \\
\hline Zinc & 369.000 & 104 & 2 & 2.17 & 2 & 0.004 \\
\hline Zirconium & $<D L$ & & & & & \\
\hline Uranium & 8485.000 & 2389 & $\geq 0$ & $\geq 0$ & & \\
\hline Nitrate & $\geq 36500.000$ & 10279 & 10279 & $\leq 100$ & 10740 & 17.16 \\
\hline Chloride & $\geq 700.000$ & 197 & 197 & $\leqslant 100$ & 206 & 0,33 \\
\hline Fluoride & $>400.000$ & 113 & 113 & $<100$ & 118 & 0.19 \\
\hline Free $\mathrm{OH}$ & NR & & & & 1667 & 2.66 \\
\hline Carbonate & $\geq 25500.000$ & 7181 & 7181 & $\leq 100$ & 7503 & 11.99 \\
\hline Organic Salts & $\geq 6630,000$ & 1867 & 1867 & $\leq 100$ & 1951 & 3.12 \\
\hline Nitrite & $\geq 39000.000$ & 10983 & 10983 & $\leq 100$ & 11475 & 18.34 \\
\hline Sulfate & $\geq 6950.000$ & 1957 & 1957 & $\leq 100$ & 2045 & 3.27 \\
\hline & & & & & & \\
\hline & & & & & & 100.00 \\
\hline Radionuclldes & $u C i / g$ & Cintank & Ciftank & & & \\
\hline Pu-239,240 & 0.448 & 126.159 & NR & & & \\
\hline C-14 & $\geq 0.000034$ & 0.010 & 0.010 & $\leq 100$ & & \\
\hline $\begin{array}{l}\text { Sr-90 } \\
\text { Tc-99 }\end{array}$ & $\begin{array}{l}1055 \\
0.101\end{array}$ & $\begin{array}{r}297092.220 \\
28.442\end{array}$ & $\begin{array}{l}\text { NR } \\
\text { NR }\end{array}$ & & & \\
\hline$A m>A 1$ & & & 1126 & & & \\
\hline Co-60 & 0.014 & 3.942 & 0.282 & 7.14 & & \\
\hline C\$-137 & 715 & 201346.860 & 2038.813 & 1.01 & & \\
\hline $1-129$ & NR & & & & & \\
\hline
\end{tabular}


C-110

\begin{tabular}{|lr|}
\hline Density (kg/L) & 1.2 \\
Volume (L)/tank & 707795 \\
Mass (kg)/tank & 849354 \\
Solids (mg/kg) & 401600 \\
Water (mg/kg) & 598400 \\
\hline
\end{tabular}

$0.1 \mathrm{M}$ NaOH Wash

Volume wash (L)/tank

(4 volume wash:1 volume sludge)

Mass removed (kg)/ank
3M NaOH Leach

$2831 \overline{180}$

528716

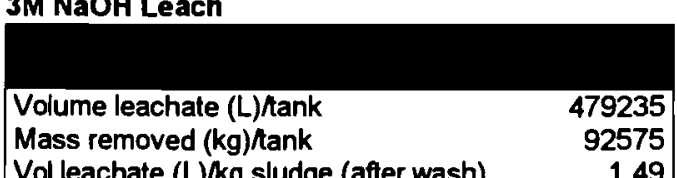

Residual Sollds

Residual solids/tank (kg) 213608

Vol leachate (L)/kg sludge (after wash)

$3 \mathrm{M}$ NaOH LEACH

-UNTREATED -

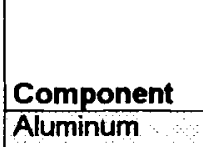

Aluminum

Barium

Bismuth

Cadmium

Calcium

Chromium

Cobalt

Copper

Iron

Lead

Magnesium

Manganese

Nickel

Phosphate

Potassium

Silicon:

Silver

Sodium.

Strontium

Zinc

Zirconium

Uranium

Nitrate

Chloride

Fluoride

Free OH

Carbonate

Organic Salts

Nitrite

Sulfate

UNTREATED -
ug/g sludge $\quad \mathrm{kg} / \mathrm{tank}$

removed

FINAL COMPOSITION

$\frac{1 \text { element) (element) }}{14560.000}$

kgtank $\%$ removed $\mathrm{mg} /$ dry w \%

\section{re}

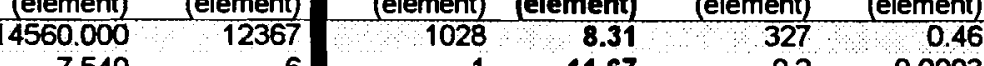

7.540

16500.000

24.900

5.200

636.000

467.000

4.110

82200

10950.000

267.000

149.000

53.000

24.200

61860.000

567.000

7050.000

4.990

83700,000

126.000

219.000

167.000

1510.000

6
14014

8.31

kg/tank \%

$\mathrm{mg} / \mathrm{L}$ dry $\mathrm{wt} \%$

$\begin{array}{rrr}4897 & \text { (element) (oxide) }\end{array}$

109080.000

$\$ 1380.000$

$\geq 7520.000$

, 10490.000

$\geq 2180.000$

$\geq 7260.000$

$\begin{array}{rrr}11.67 & 0.2 & 0.0003 \\ 0.71 & 32 & 0.04\end{array}$

489

ide)

$\begin{array}{rrr}21 & 21 & 99.84 \\ 4 & 1 & 15.62\end{array}$

29.53

55.59

66.91

2.84

2.84

7.43

7.43
9.51
3.92

11.48

38.86

69.97

3.41

23.01

79.05

4.39

5.14
7.45
$>0$

5.14
$\geq 0$
5100

$\leq 100$

$\$ 100$

$\leq 100$

$92648 \quad 92648$

6387

6387

$\leq 100$

8910

8910

$\leq 100$

$6166 \leq 100$

32
7

0.04
0.01
0.0003

$>19050.000 \quad 1618$

16180

$\leq 100$

51

0.0003
0.07

Water

598400.000

508253

316384

62.25

0.001

0.001
0.0009

$\begin{array}{rr}1 & 0.0009 \\ 84 & 0.12 \\ 5 & 0.01\end{array}$

$\begin{array}{rr}4 & 0.01 \\ 1 & 0.0008\end{array}$

0.0008

$6487 \quad 9.13$

$107 \quad 0.15$

0.15
0.09
0.0004

$\begin{array}{ll}0.3 & 0.0004\end{array}$

$1 \quad 0.002$

0.002

0.004
0.005

$29435 \quad 41.42$

$372 \quad 0.52$

$2029 \quad 2.86$

$1529 \quad 215$

$2831 \quad 3.98$

$\begin{array}{ll}580 & 0.83 \\ 1959 & 2.76\end{array}$

$5141+723$

Radionuclides

Pu-239,240

C-14

Sr.90

Tc-99

Am-241.

Co-60

Cs-137

I-129

\begin{tabular}{rr} 
uCi/g & Cintank \\
0,08 & 67.948 \\
20.005 & 4.247 \\
4.98 & 4229.783 \\
0.035 & 29.727 \\
& \\
0.005 & 4247 \\
$<0.04$ & $<34$ \\
19.4 & 16477.468 \\
$<0.019$ & $<16$ \\
\hline
\end{tabular}

Ci/tank

$4.247 \leq 100.00$

NR

NR

NR

NR

31911

55397

10.90

100.00

100.00

\begin{tabular}{lll|l}
31220 & 59.42 & 58396 & 28.53
\end{tabular}

61850

54.52

RESIDUAL SOLIDS

kghank mg/kg res dry wt\%

$\begin{array}{rrr}\text { element) } & \text { (oxide) } & \text { (oxide) } \\ 6442 & 56964 & 15.77\end{array}$

$\begin{array}{rrr}6442 & 56964 & 15.77 \\ 6 & 30 & 0.01\end{array}$

$\begin{array}{rrr}6 & 30 & 0.01 \\ 13914 & 72630 & 20.11\end{array}$

$\begin{array}{rrr}13914 & 72630 & 20.11 \\ 0.03 & 1 & 0.0001 \\ 4 & 20 & 0.01 \\ 381 & 2493 & 0.69\end{array}$

$\begin{array}{rrr}44 & 301 & 0.08 \\ 1 & 8 & 0.002 \\ 68 & 398 & 0.11\end{array}$

$\begin{array}{rrr}68 & 398 & 0.11 \\ 9037 & 60498 & 16.75 \\ 210 & 1058 & 0.29 \\ 115 & 889 & 0.25\end{array}$

$43 \quad 320 \quad 0.09$

$\begin{array}{ll}3152 & 0.03 \\ 677 & 0.87\end{array}$

$\begin{array}{rrr}145 & 677 & 0.19 \\ 5784 & 57918 & 16.04\end{array}$

$\begin{array}{rrr}5784 & 57918 & 16.04\end{array}$

$\begin{array}{rrr}4891 & 93971 & 0.00 \\ 102 & 567 & 0.02\end{array}$

$\begin{array}{rrr}102 & 567 & 0.16\end{array}$

$\begin{array}{rrr}176 & 1028 & 0.28 \\ 131 & 830 & 0.23\end{array}$

$\begin{array}{rrr}131 & 830 & 0.23 \\ 1283 & 7217 & 2.00\end{array}$

$\begin{array}{lll}\geq 0 & \geq 0 & \geq 0.00\end{array}$

$\geq 0 \quad \geq 0.00$

$\geq 0.00$

$\geq 0.00$

$\geq 0.00$

$\geq 0.00$

$\geq 0.00$

100.00 


\section{C-112}

Density (kg/L)

Volume (L)Mank

Mass (kg) hank

Solids (mg/kg)

Water (mg/kg)

415000

$0.1 \mathrm{M}$ NaOH Wash

Volume wash (L)/tank

(4 volume wash:1 volume sludge)

Mass removed $(\mathrm{kg})$ /ank
$3 \mathrm{M} \mathrm{NaOH}$ Leach

1574560

341049
Residual Sollds

Residual solids^ank (kg)

$223 \overline{71}$

Mass removed (kg)/hank

Vol leachate (L)/kg sludge (after wash)

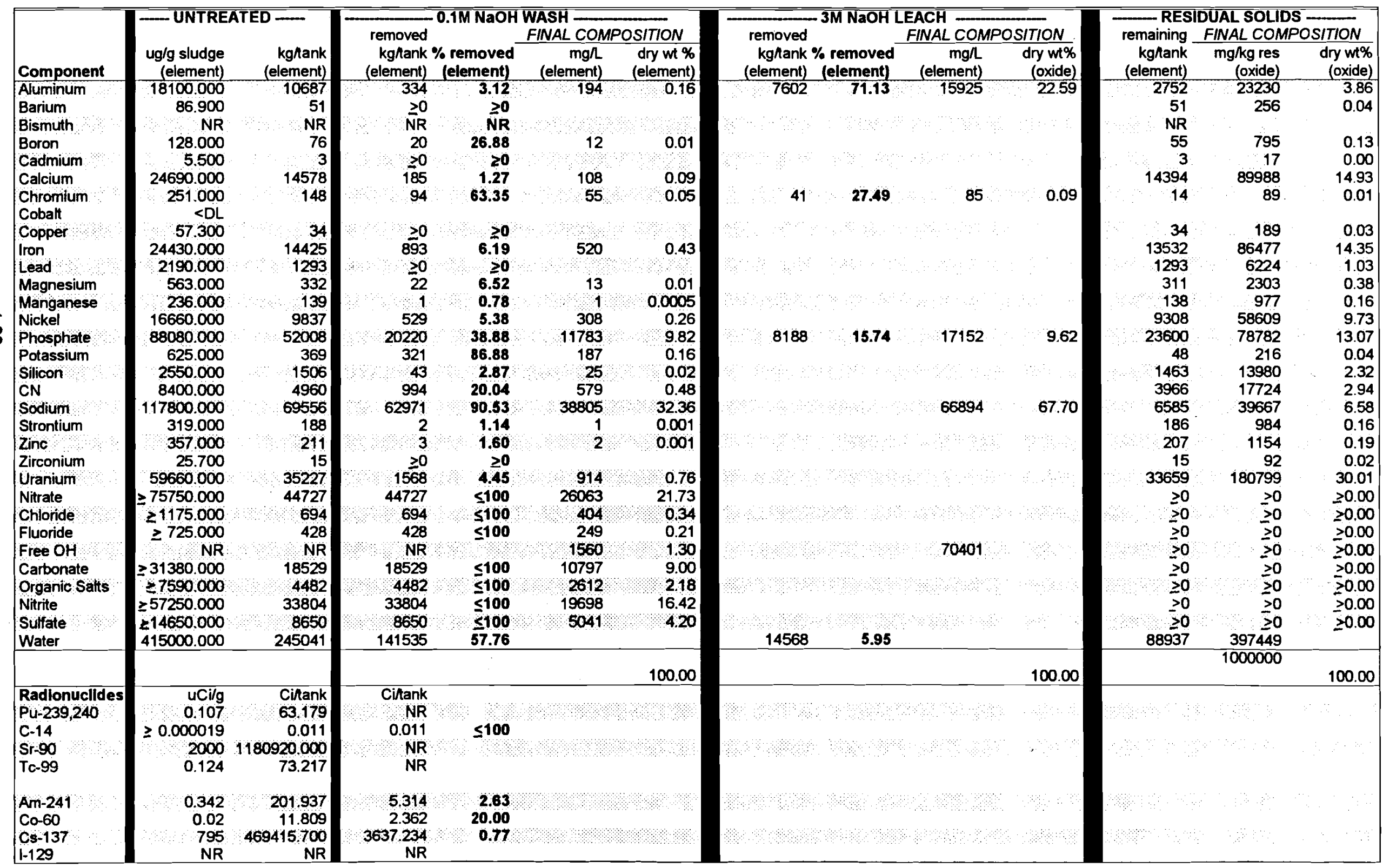


S-104

Density (kg几)

Volume (L) Hank

Mass (kg)/tank

Solids (mg/kg)

Water (mg/kg)

- UNTREATED -

Component

Aluminum

Barium

Bismuth

Boron

Cadmium

Calcium

Chromium

Cobalt

Copper

Iron

Lead

Magnesium

Manganese

Nickel

Phosphate

Potassium

Silicon

Sodium

Strontium

Zinc

Zirconium

Uranium

Nitrate

Chloride

Fluoride

Free $\mathrm{OH}$

Carbonate

Organic Salts

Nitrite

Sulfate

Water

Radionuclides

Pu-239,240

C-14

Sr-90

Tc-99

Am-241.

Co-60

Co-60

Cs-137
I-129

\begin{tabular}{|c|c|c|c|c|c|}
\hline \multirow{2}{*}{\multicolumn{2}{|c|}{ - UNTREATED - }} & \multicolumn{4}{|c|}{$0.1 \mathrm{M} \mathrm{NaOH}$ WASH _- } \\
\hline & & \multicolumn{4}{|c|}{$\begin{array}{ll}\text { removed } & \text { FINAL COMPOSITION }\end{array}$} \\
\hline $\begin{array}{l}\text { ug/g sludge } \\
\text { (element) }\end{array}$ & $\begin{array}{r}\text { kg/tank } \\
\text { (element) }\end{array}$ & $\begin{array}{l}\text { kg/tank } \\
\text { (element) }\end{array}$ & $\begin{array}{l}\% \text { removed } \\
\text { (element) }\end{array}$ & $\begin{array}{r}\mathrm{mg} / \mathrm{L} \\
\text { (element) }\end{array}$ & $\begin{array}{l}\text { dry wt \% } \\
\text { (element) }\end{array}$ \\
\hline 118000.000 & 196294 & 7872 & 4.01 & 1663 & 137 \\
\hline 33.300 & 55 & 1 & 0.93 & 0.1 & 0.00009 \\
\hline$<D L$ & & & & & \\
\hline 39.200 & 65 & 15 & 22.91 & 3 & 0.003 \\
\hline 1910.000 & 3177 & 164 & 5.17 & 35 & 0.03 \\
\hline 4290.000 & 7136 & 3184 & $\mathbf{4 4 . 6 2}$ & 673 & 0.55 \\
\hline 6.730 & 11 & 2 & 17.83 & 0.4 & 0.0003 \\
\hline 51.800 & 86 & 1 & 1.64 & 03 & 0,0002 \\
\hline 2490.000 & 4142 & 6 & 0.13 & 1 & 0.001 \\
\hline 38.500 & 64 & 14 & 21.17 & 3 & 0.002 \\
\hline 53.500 & 89 & 7 & 8.37 & 2 & 0.001 \\
\hline 1090.000 & 1813 & 1 & 0.03 & 0.1 & 0.00009 \\
\hline 4880.000 & 8118 & 3 & 0.03 & 1 & 0.0005 \\
\hline 289.000 & 481 & 45 & 9.43 & 10 & 0.01 \\
\hline 300.000 & 499 & 421 & 84.27 & 89 & 0.07 \\
\hline 1460.000 & 2429 & 57 & 2.36 & 12 & 0.01 \\
\hline$<D L$ & & & & & \\
\hline 119333.000 & 198511 & 151185 & 76.16 & 34096 & 28.07 \\
\hline 418.000 & 695 & 1 & 0.15 & 0.2 & 0.0002 \\
\hline 325.000 & 541 & 21 & 3.82 & 4 & 0.004 \\
\hline 645.000 & 1073 & 2 & 0.16 & 0.4 & 0.0003 \\
\hline 6620000 & 11012 & $\geq 0$ & $\geq 0$ & & \\
\hline 185700.000 & 308913 & 308913 & $\leq 100$ & 65263 & 53.73 \\
\hline$\geq 3170000$ & 5273 & 5273 & $\leq 100$ & 1114 & 0,92 \\
\hline$\geq 145.000$ & 241 & 241 & $\leq 100$ & 51 & 0.04 \\
\hline NR & & & & 1593 & 1.31 \\
\hline$\geq 20970.000$ & 34884 & 34884 & $\leq 100$ & 7370 & 6.07 \\
\hline$\geq 3760.000$ & 6255 & 6255 & $\leq 100$ & 1321 & 1.09 \\
\hline$\geq 20820.000$ & 34634 & 34634 & $\leq 100$ & 7317 & 6.02 \\
\hline$\geq 2370.000$ & 3943 & 3943 & $\leq 100$ & 833 & 0.69 \\
\hline 348000.000 & 578901 & 297371 & 51.37 & & \\
\hline & & & & & 100.00 \\
\hline & Cirtank & Cintank & & & \\
\hline 0.284 & 472.436 & NR & & & \\
\hline$=0.001$ & 1.664 & 1.664 & $\leq 100$ & & \\
\hline 303 & 504042.773 & NR & & & \\
\hline 0.025 & 41.588 & NR & & & \\
\hline & 196294 & NR & & & \\
\hline 0.033 & 54.896 & NR & & & \\
\hline 63.2 & 105133.674 & NR & & & \\
\hline 0.013 & 21.626 & NR & & & \\
\hline
\end{tabular}

$0.1 \mathrm{M} \mathrm{NaOH}$ Wash

Volume wash (L) hank

(4 volume wash:1 volume sludge)

Mass removed $(\mathrm{kg})$ /ank
$3 \mathrm{M} \mathrm{NaOH}$ Leach

4436020

1109005

663508 348000
854513
Residual Sollds

Residual solidshank (kg)

222507

Volume leachate (L)/Aank $\quad 2356119$

683672
2.91

Vol leachate $(L) / k g$ sludge (after wash) $\quad 2.91$

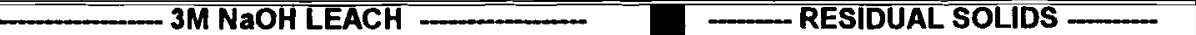

removed FINAL COMPOSITION

$\mathrm{kg}$ tank \% removed $\frac{\mathrm{mg} / \mathrm{L} \quad \mathrm{dry} w \mathrm{w} \%}{2}$

\begin{tabular}{rrrr} 
(element) & (element) & (element) & (oxide) \\
\hline 158215 & $\mathbf{8 0 . 6 0}$ & 60992 & 66.39
\end{tabular}

remaining FINAL COMPOSITION

$\mathrm{kghank} \mathrm{mg} / \mathrm{kg}$ res dry $\mathrm{wt} \%$

\begin{tabular}{rrr} 
(element) & (oxide) & (oxide) \\
\hline 30207 & 301383 & 37.49 \\
55 & 275 & 0.03
\end{tabular}

$\begin{array}{rrr}55 & 275 & 0.03\end{array}$

50

727

0.09

2964

41.54

1143

18945
6493

2.36

$\begin{array}{llll}988 & 6493 & 0.81\end{array}$

85

4137
50

58
477

26585

0.01

26585
244

0.06
3.31
0.03

38.500

0.002

00009

$\geq 0 \geq 0.00$

82

82
1813
8115

$\begin{array}{rrr}8115 & 51388 & 6.39 \\ 435 & 1462 & 0.18 \\ 79 & 353 & 0.04\end{array}$

608
12888

51388

0.03
0.08

$\begin{array}{rrr}8115 & 51388 & 6.39 \\ 435 & 1462 & 0.18 \\ 79 & 353 & 0.04\end{array}$

1.60
6.39

$\begin{array}{rrr}2371 & 22798 & 0.04 \\ & & 2.84\end{array}$

$62672 \quad 32.96$

237918

41.10

$47327 \quad 286717 \quad 35.66$

$\begin{array}{lll}694 & 3691 & 0.46\end{array}$

520

1071
11012

2910

6504
59490

0.36
0.81

0.81
7.40

70.00

162650

\begin{tabular}{lll}
237918 & 41.10 & \\
& 100.00 \\
\hline
\end{tabular}


T-102

Density (kg/L)

Volume (L) Mank

Mass (kg)/ank

Solids (mg/kg)

Water (mg/kg)
$0.1 \mathrm{M} \mathrm{NaOH}$ Wash

Volume wash (L) tank

(4 volume wash:1 volume sludge)

Mass removed $(\mathrm{kg})$ hank
3M NaOH Leach

\begin{tabular}{|lr|}
\hline & \\
\hline Volume leachate (L)/hank & 354448 \\
Mass removed $(\mathrm{kg}) /$ /ank & 62705 \\
Vol leachate $(\mathrm{L}) / \mathrm{kg}$ sludge (after wash) & 3.03 \\
\hline
\end{tabular}

Residual Sollds

Residual solids/tank (kg)

$274 \overline{30}$

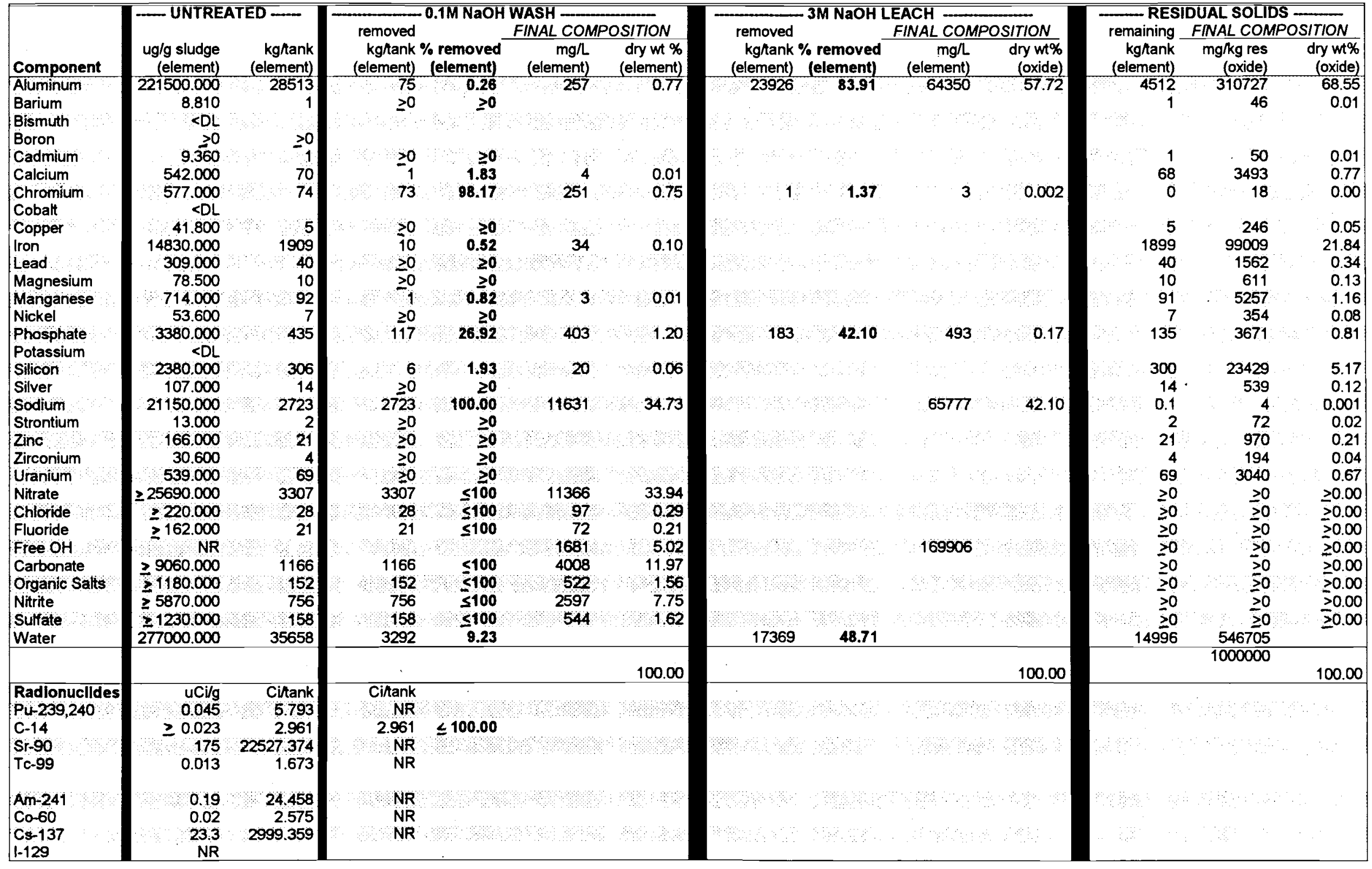


T-104

\begin{tabular}{|lr|}
\hline Density (kg/L) & 1.15 \\
Volume (L)/tank & 1672970 \\
Mass (kg)hank & 1923916 \\
Solids (mg/kg) & 293500 \\
Water (mg/kg) & 706500 \\
\hline
\end{tabular}

$1 \mathrm{M} \mathrm{NaOH} \mathrm{Wash}$

Volume wash (L)/tank

(4 volume wash:1 volume sludge)

Mass removed (kg)Mank

6691880

930086

$3 \mathrm{M} \mathrm{NaOH}$ Leach

$\begin{array}{lr}\text { Volume leachate (L)/Aank } & 1499092 \\ \text { Mass } & 373149\end{array}$

$\begin{array}{lr}\text { Mass removed (kg)/ank } & 373149 \\ \text { Vol leachate (L)/kg sludge (after wash) } & 1.51\end{array}$
Residual Sollds

Residual solids/ank (kg)

610519

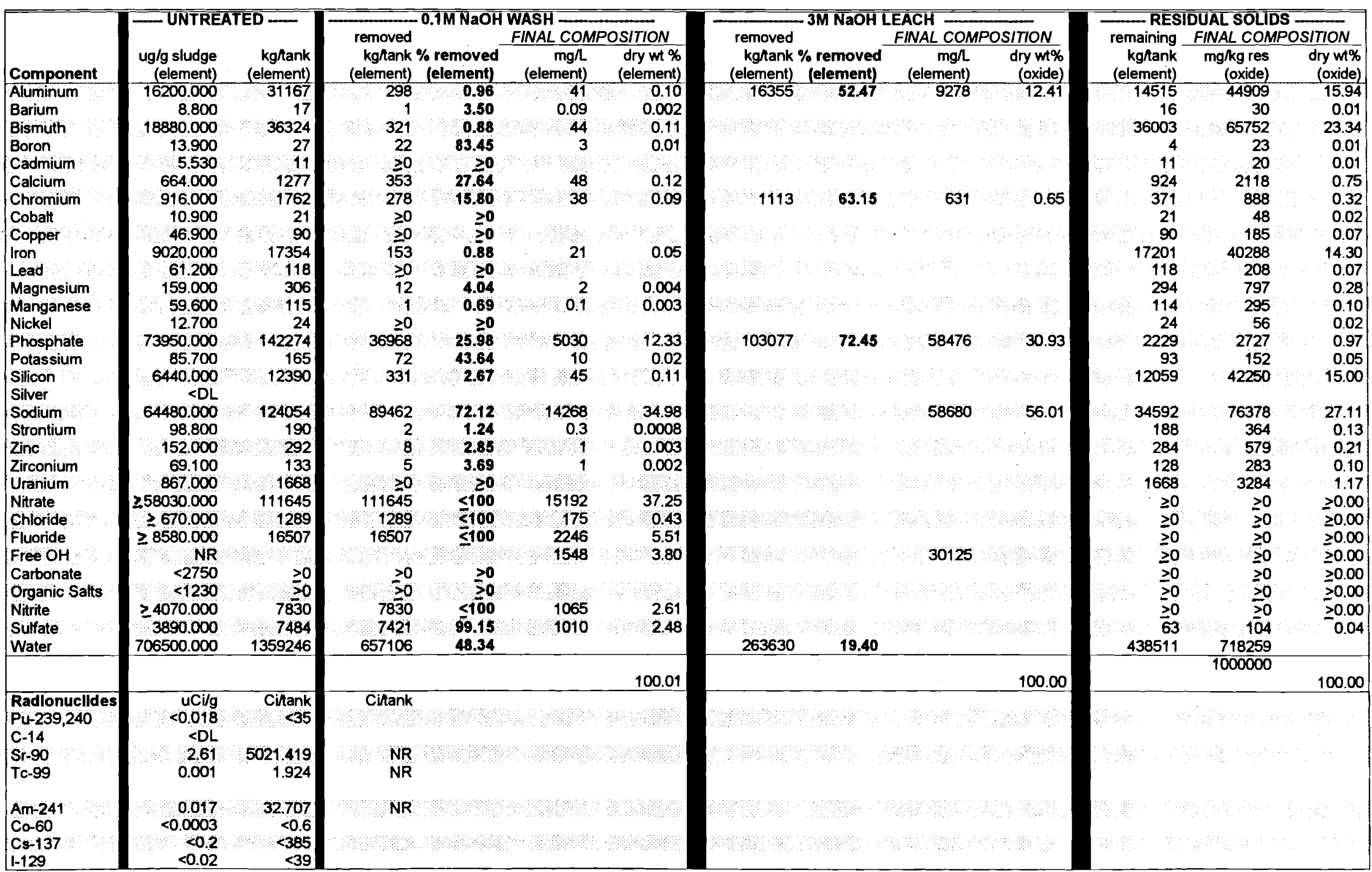


T-107

\begin{tabular}{|lr|}
\hline Density (kg/L) & 1.5 \\
Volume (L)/tank & 647235 \\
Mass (kg)/tank & 970853 \\
Solids (mg/kg) & 501750 \\
Water (mg/kg) & 498250 \\
\hline
\end{tabular}

0.1 M NaOH Wash

Volume wash (L) Mank

(4 volume wash:1 volume sludge)

Mass removed (kg)/tank
3M NaOH Leach

2588940

581443

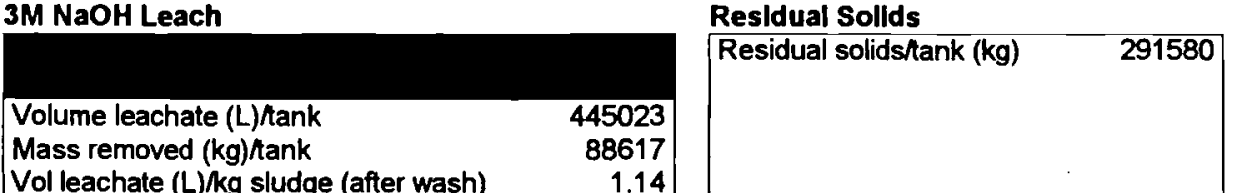

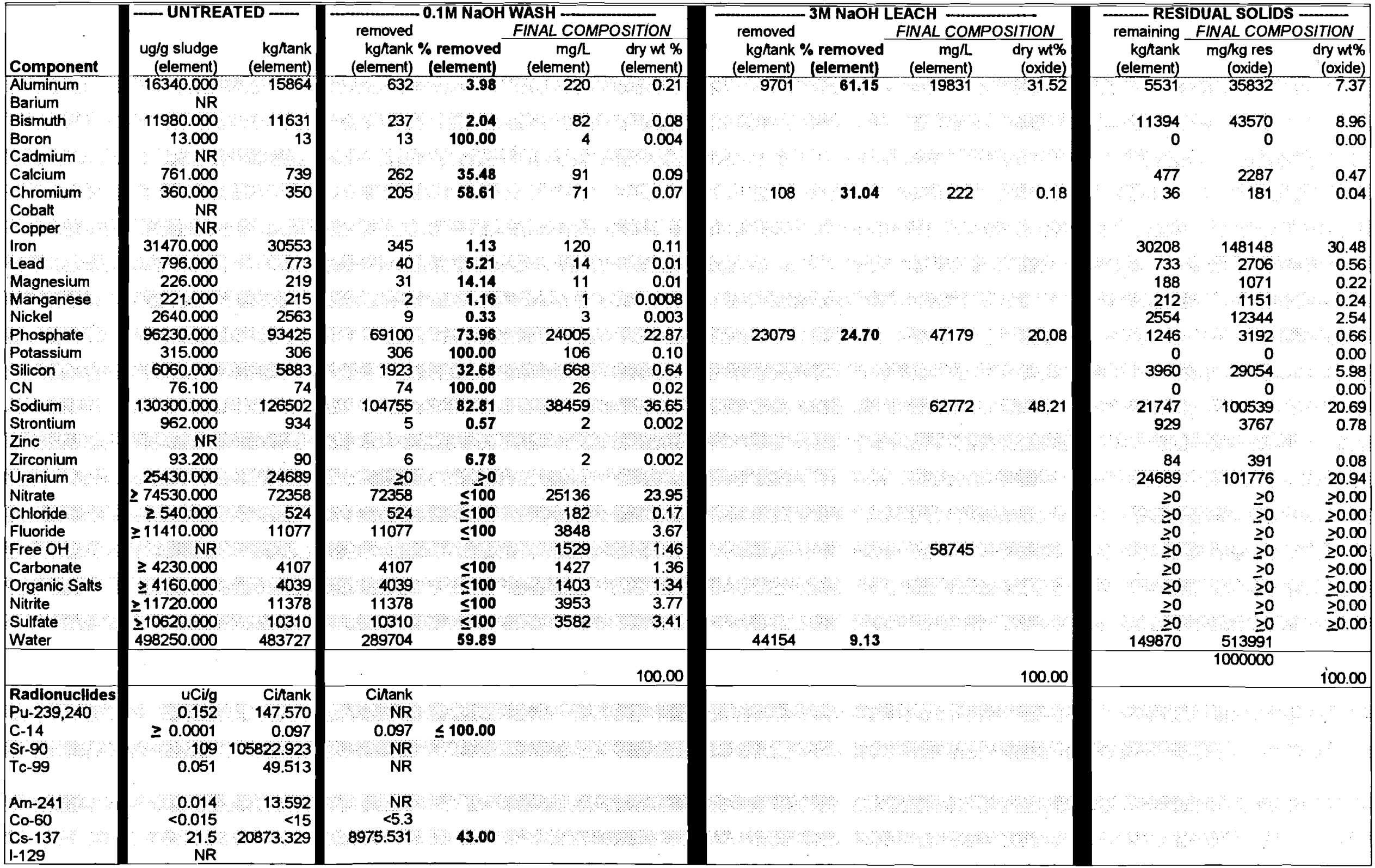




T-111
\begin{tabular}{|lr|}
\hline Density (kg/L) & 1.1 \\
Volume (L)/ank & 1714605 \\
Mass (kg)/ank & 1886066 \\
Solids (mg/kg) & 239800 \\
Water (mg/kg) & 760200 \\
\hline
\end{tabular}

$0.1 \mathrm{M} \mathrm{NaOH}$ Wash

Volume wash (L) hank

(4 volume wash:1 volume sludge)

Mass removed $(\mathrm{kg})$ /hank
3M NaOH Leach

6858420

881201
Volume leachate (L)/tank

Mass removed $(\mathrm{kg})$ Mank

Vol leachate (L)/kg sludge (after wash)
Residual Sollds

Residual solids/tank (kg) $\quad 899789$

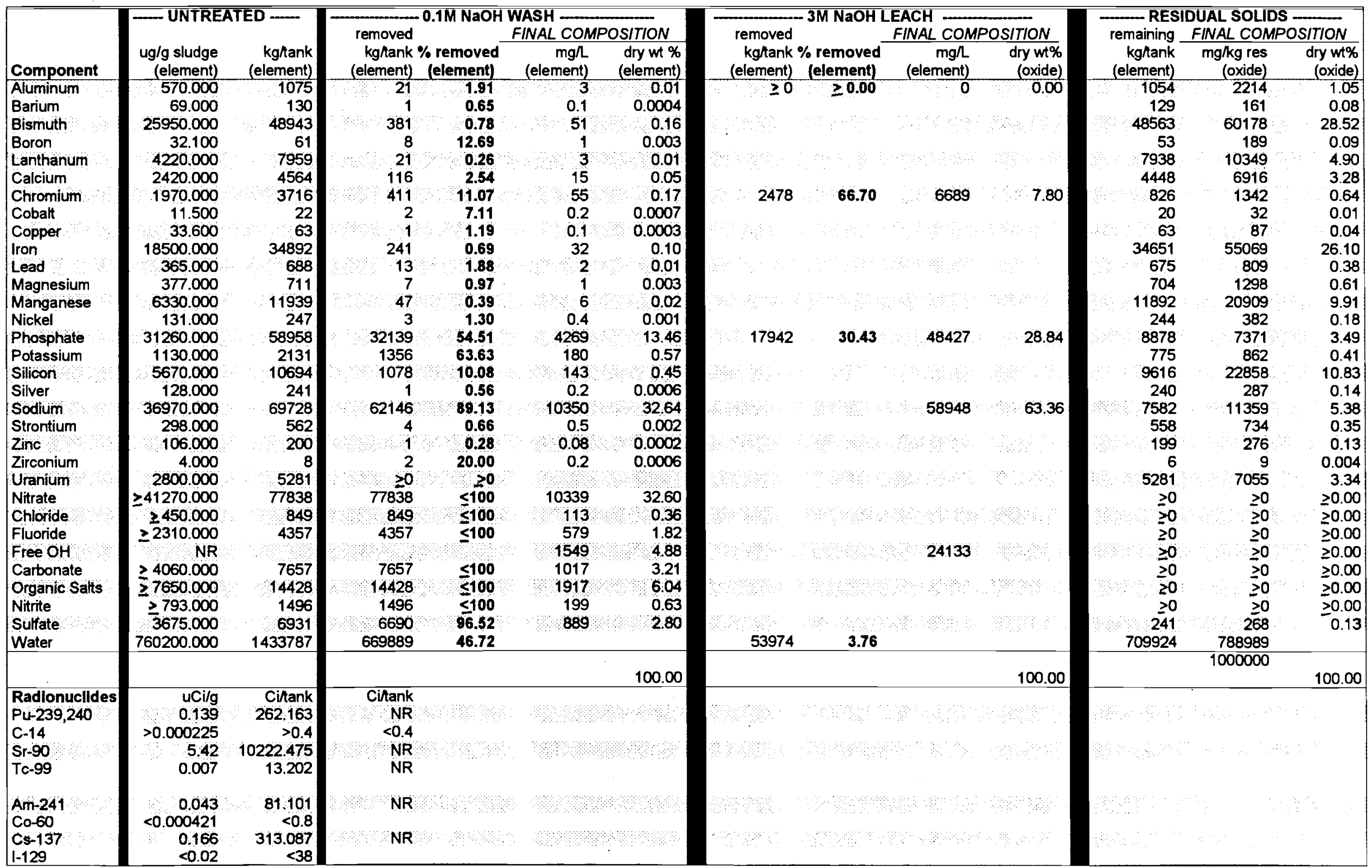




TY-101
\begin{tabular}{|lr|}
\hline Density (kg/L) & 1.64 \\
Volume (L)/ank & 446630 \\
Mass (kg)/ank & 732473 \\
Solids (mg/kg) & 565000 \\
Water (mg/kg) & 435000 \\
\hline
\end{tabular}

$0.1 \mathrm{M}$ NaOH Wash

Volume wash (L)/tank

(4 volume wash:1 volume sludge)

Mass removed (kg)/tank

1786520

320696

3M NaOH Leach

\begin{tabular}{|lr|}
\hline & \\
\hline Volume leachate (L)/Aank & 752055 \\
Mass removed (kg)/Aank & 68986 \\
Vol leachate (L)/kg sludge (after wash) & 1.83 \\
\hline
\end{tabular}
Residual Sollds

Residual solidsfank (kg) $\quad 372418$

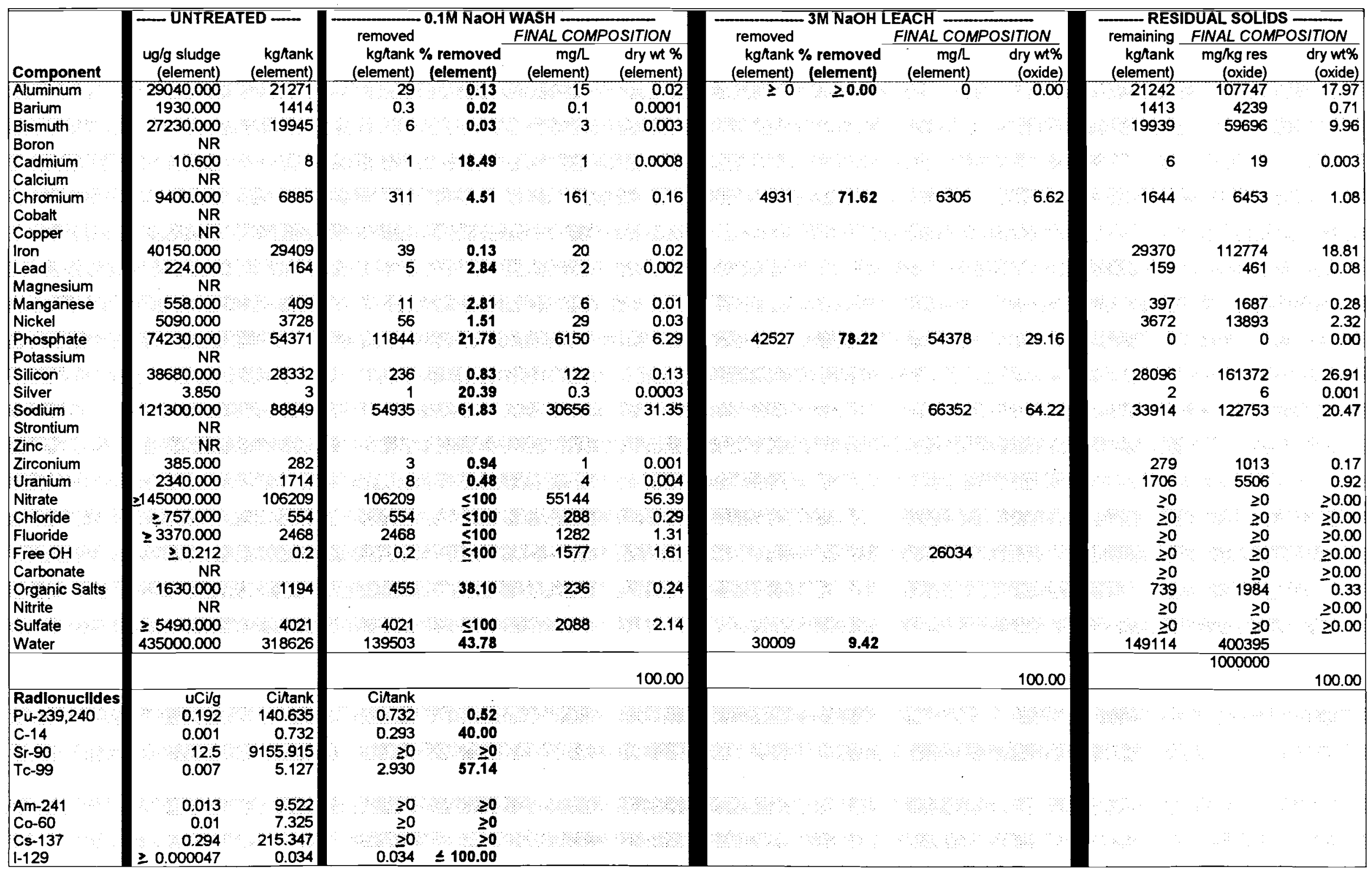


TY-103

Density (kg/L) Volume (L) tank Mass $(\mathrm{kg})$ Hank Solids (mg/kg) Water $(\mathrm{mg} / \mathrm{kg})$
$0.1 \mathrm{M}$ NaOH Wash

Volume wash (L)/tank

( 4 volume wash:1 volume sludge)

Mass removed (kg)/ank
$3 \mathrm{M} \mathrm{NaOH} \mathrm{Leach}$

591029

Residual Sollds

Residual solidstank (kg) $\quad 3 \overline{82033}$

Volume leachate (L)/ank $\quad 591947$

Mass removed (kg)/tank 73493

1.31

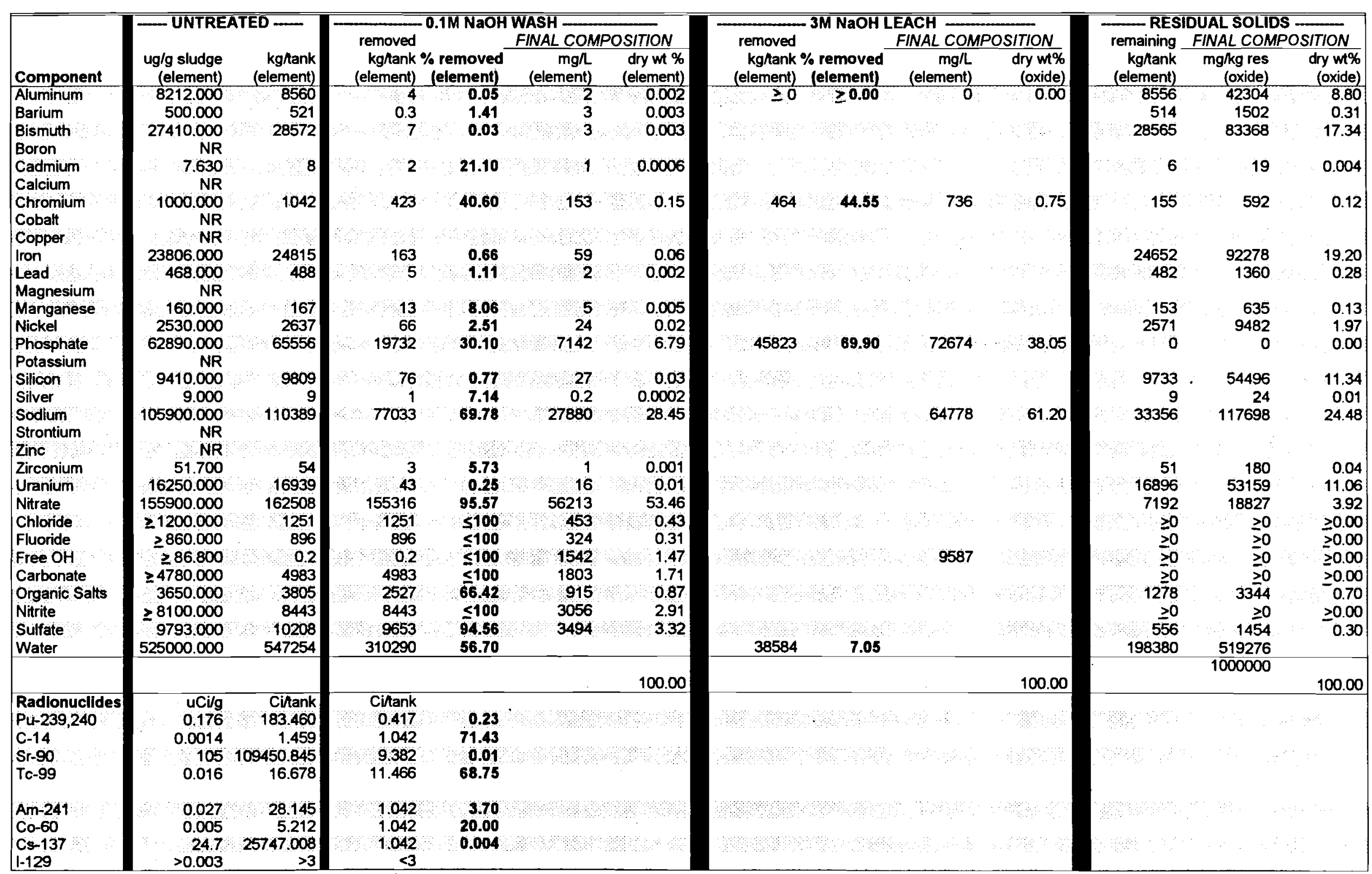


TY-104

\begin{tabular}{|lr|}
\hline Density $(\mathrm{kg} / \mathrm{L})$ & 1.69 \\
Volume (L) & 162755 \\
Mass (kg)/hank & 294246 \\
Solids (mg/kg) & 430250 \\
Water (mg/kg) & 569750 \\
\hline
\end{tabular}

$0.1 \mathrm{M} \mathrm{NaOH}$ Wash

Volume wash (L) hank

(4 volume wash:1 volume sludge)

Mass removed (kg)/tank
$3 \mathrm{M} \mathrm{NaOH}$ Leach

651020

\begin{tabular}{|lr|}
\hline & \\
\hline Volume leachate (L)/Aank & 110686 \\
Mass removed $(\mathrm{kg})$ /Aank & 14417 \\
Vol leachate $(\mathrm{L}) \mathrm{kg}$ sludge (after wash) & 0.82 \\
\hline
\end{tabular}

Residual Sollds

Residual solids/tank (kg) 118397

0.82

Vol leachate $(\mathrm{L}) / \mathrm{kg}$ sludge (after wash)

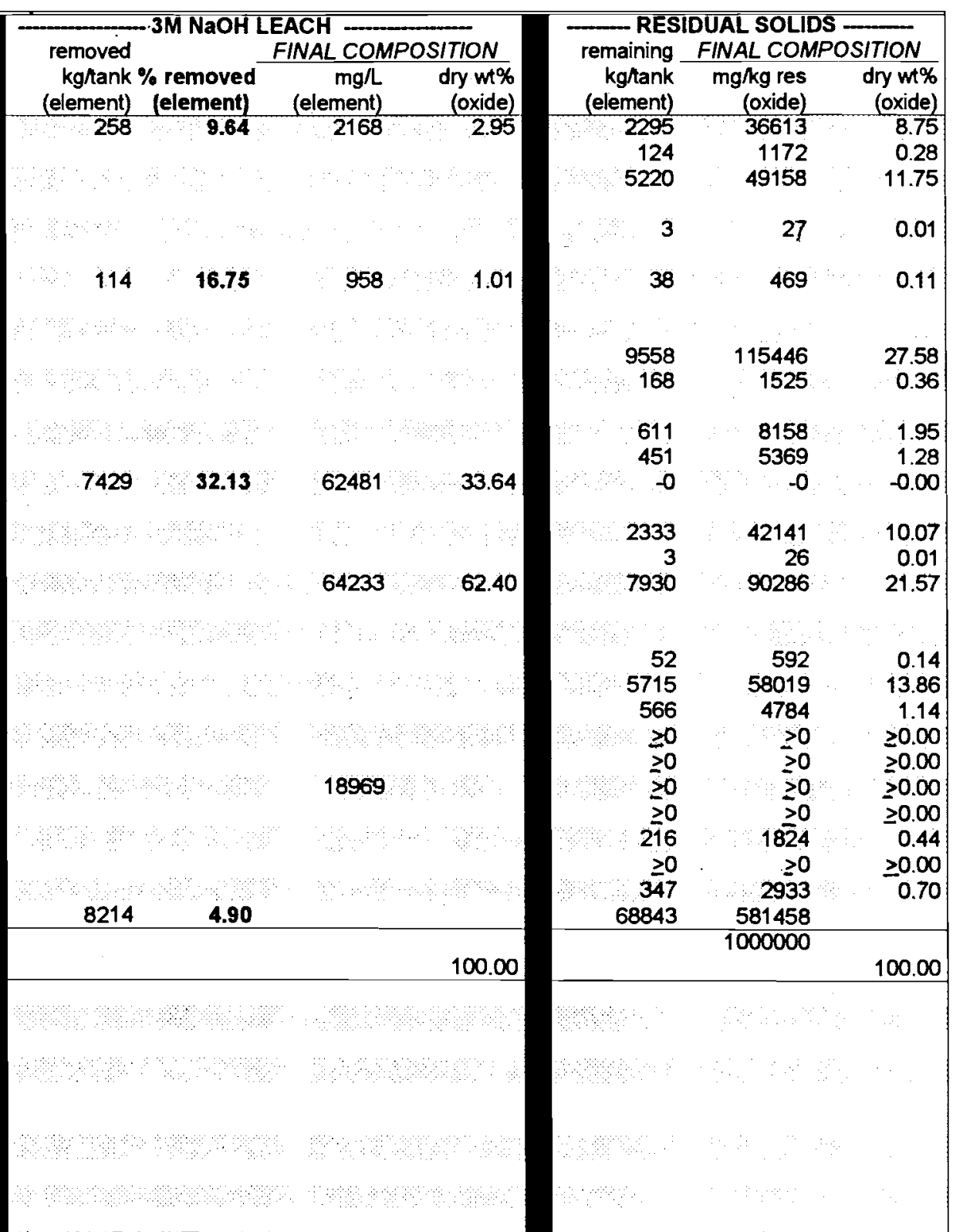

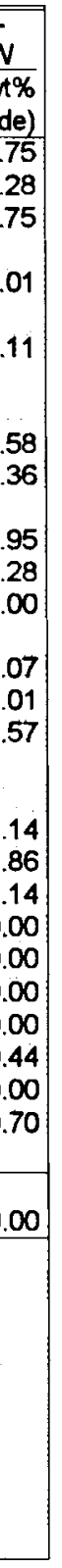

\begin{tabular}{|c|c|c|}
\hline & - UNTR & IED - \\
\hline Componer & $\begin{array}{l}u g / g \text { sludge } \\
\text { (element) }\end{array}$ & $\begin{array}{r}\mathrm{kg} \text { /tank } \\
\text { (element) }\end{array}$ \\
\hline Aluminum & 9090.000 & 2675 \\
\hline Barium & 423.000 & 124 \\
\hline Bismuth & 17750.000 & 5223 \\
\hline Boron & $\begin{array}{l}\text { NR } \\
8 M\end{array}$ & \\
\hline $\begin{array}{l}\text { Cadmium } \\
\text { Calcium }\end{array}$ & $\begin{array}{r}12.800 \\
\text { NR }\end{array}$ & 4 \\
\hline Chromium & 2310.000 & 680 \\
\hline $\begin{array}{l}\text { Cobalt } \\
\text { Copper: }\end{array}$ & $\begin{array}{l}\text { NR } \\
\text { NR }\end{array}$ & \\
\hline $\begin{array}{l}\text { Lopper. } \\
\text { Iron }\end{array}$ & 32570.000 & 9584 \\
\hline Lead & 580.000 & 171 \\
\hline Magnesium & NR & \\
\hline $\begin{array}{l}\text { Manganese } \\
\text { Nickel }\end{array}$ & $\begin{array}{l}2090.000 \\
1550.000\end{array}$ & $\begin{array}{l}615 \\
456\end{array}$ \\
\hline Phosphate & 78580.000 & 23122 \\
\hline Potassium & NR & \\
\hline Silicon & 8090.000 & 2380 \\
\hline Silver & 12.400 & \\
\hline $\begin{array}{l}\text { Sodium } \\
\text { Strontium }\end{array}$ & $\begin{array}{r}113100.000 \\
\text { NR }\end{array}$ & 33279 \\
\hline Zinc & NR & \\
\hline Zirconium & 181.000 & 53 \\
\hline Uranium & 19460.000 & 5726 \\
\hline Nitrate & 46600.000 & 13712 \\
\hline Chloride & $\geq 598.000$ & 176 \\
\hline Fluoride & $\geq 5350.000$ & 1574 \\
\hline Free OH & $\geq 1290.000$ & 380 \\
\hline Carbonate & $>18250.000$ & 5370 \\
\hline Organic Salts & 4750.000 & 1398 \\
\hline Nitrite & $\geq 12220.000$ & 3596 \\
\hline Sulfate & 5230.000 & 1539 \\
\hline Water & 569750.000 & 167647 \\
\hline \begin{tabular}{|l|} 
Radionuclldes \\
\end{tabular} & $\mathrm{uCi} / \mathrm{g}$ & Ciftank \\
\hline Pu-239;240 & 0.181 & 53.259 \\
\hline C-14 & 0.003 & 0.883 \\
\hline Si-90 & 132 & 38840.472 \\
\hline Tc-99 & 0.033 & 9.710 \\
\hline Am-241 & 0.034 & 10.004 \\
\hline Co-60 & $\begin{array}{r}0.023 \\
454\end{array}$ & $\begin{array}{r}6.768 \\
58768\end{array}$ \\
\hline $\begin{array}{l}\text { Cs-137 } \\
1-129\end{array}$ & $\begin{array}{r}45.4 \\
00335\end{array}$ & $\begin{array}{r}13358.768 \\
>0.010\end{array}$ \\
\hline
\end{tabular}

\begin{tabular}{|c|c|c|c|}
\hline & $0.1 \bar{M} \mathrm{NaOH}$ & NASH - & $\bar{E}$ \\
\hline removed & & FINAL COM & OSITION \\
\hline $\begin{array}{c}\mathrm{kg} / \text { tank } \\
\text { (element) }\end{array}$ & $\begin{array}{l}\% \text { removed } \\
\text { (element) }\end{array}$ & $\begin{array}{r}\mathrm{mg} / \mathrm{L} \\
\text { (element) }\end{array}$ & $\begin{array}{l}\text { dry } w t \% \\
\text { (element) }\end{array}$ \\
\hline 122 & 4.57 & $\frac{-155}{155}$ & 0.17 \\
\hline 0.3 & 0.22 & 0.4 & 0.0004 \\
\hline 3 & 0.06 & 4 & 0.004 \\
\hline 1 & 24.53 & 1 & 0.001 \\
\hline 528 & 77.66 & 671 & 0.74 \\
\hline $\begin{array}{r}25 \\
3\end{array}$ & $\begin{array}{l}0.26 \\
1.77\end{array}$ & 32 & $\begin{array}{r}0.04 \\
0.004\end{array}$ \\
\hline & 0.72 & 6 & 0.01 \\
\hline 15693 & 67.87 & 19939 & 22.04 \\
\hline 48 & 2.01 & 61 & 0.07 \\
\hline 1 & 22.46 & 1 & 0.001 \\
\hline 25349 & 76.17 & 34244 & 37.86 \\
\hline 1 & 2.60 & 2 & 0.002 \\
\hline 11 & 0.20 & 14 & 0.02 \\
\hline 13145 & 95.87 & 16703 & 18.46 \\
\hline 176 & $\leq 100$ & 224 & 0.25 \\
\hline 1574 & $\leq 100$ & 2000 & 2.21 \\
\hline 380 & $\leq 100$ & 1987 & 2.20 \\
\hline 5370 & $\leq 100$ & 6823 & 7.54 \\
\hline 1182 & 84.55 & 1501 & 1.66 \\
\hline 3596 & $\leq 100$ & 4569 & 5.05 \\
\hline 1192 & 77.44 & 1514 & 1.67 \\
\hline 90590 & 54.04 & & \\
\hline & & & 100.00 \\
\hline Ciltank & & & \\
\hline 0.294 & 0.55 & & \\
\hline 0.588 & 66.67 & & \\
\hline 11.476 & 0.03 & & \\
\hline 7.062 & 72.73 & & \\
\hline $\begin{array}{l}0.588 \\
0.883\end{array}$ & $\begin{array}{r}5.88 \\
13.04\end{array}$ & & \\
\hline 4855.059 & 36.34 & & \\
\hline$<0.010$ & $\leq 100$ & & \\
\hline
\end{tabular}


TY-106

\begin{tabular}{|lr|}
$\mid Y-106$ \\
\hline Density (kg/L) & 1.37 \\
Volume (L)/tank & 64345 \\
Mass (kg)/ank & 88153 \\
Solids (mg/kg) & 638500 \\
Water (mg/kg) & 361500 \\
\hline
\end{tabular}

$0.1 \mathrm{M} \mathrm{NaOH}$ Wash

Volume wash (L) hank

(4 volume wash:1 volume sludge)

Mass removed $(\mathrm{kg})$ /ank
$3 \mathrm{M} \mathrm{NaOH}$ Leach

257380

39095
Residual Sollds

Residual solids/ank (kg)

$44 \overline{24}$

Volume leachate (L)tank 49552

Mass removed (kg)/tank 4692

Vol leachate $(\mathrm{L}) / \mathrm{kg}$ sludge (after wash)

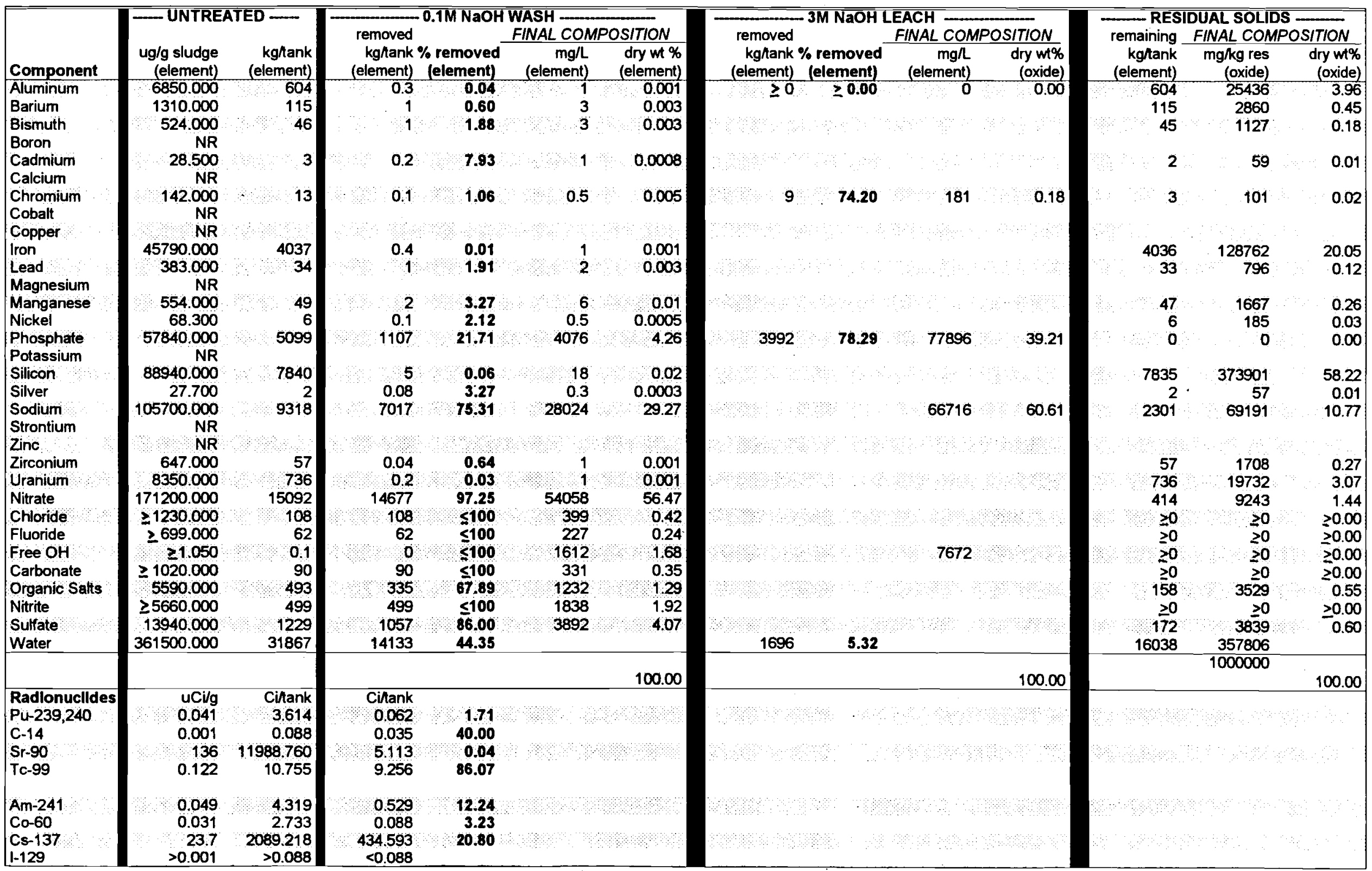




\section{U-110}

Density (kg/)

Volume (L)hank $\quad 704010$

Mass (kg)/Aank

Solids (mg/kg)

Water $(\mathbf{m g} / \mathrm{kg}$ )
$0.1 \mathrm{M}$ NaOH Wash

Volume wash (L)hank

(4 volume wash: 1 volume sludge)

Mass removed (kg)/tank
$3 \mathrm{M} \mathrm{NaOH}$ Leach

2816040

265774
Resldual Solids

Residual solids/tank (kg)

322799

2158521

Mass removed (kg) Mank

\begin{tabular}{lr} 
\\
$V$ ol leachate $(L) / k g$ sludge (after wash) & 356299 \\
\hline
\end{tabular}

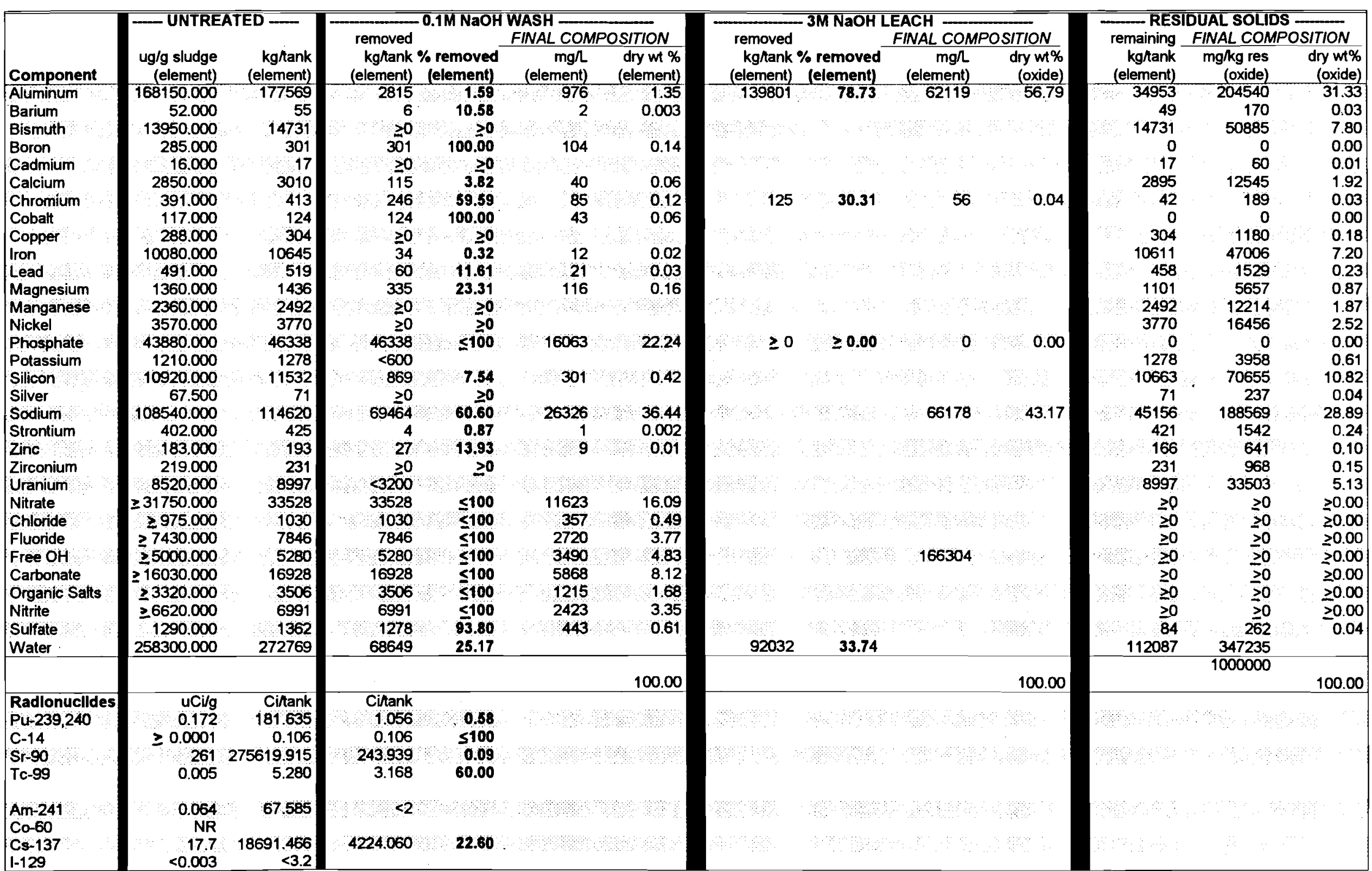


Enhanced Sludge Washing Spreadsheet Evaluation Data Summaries for 15 Different Waste Types 


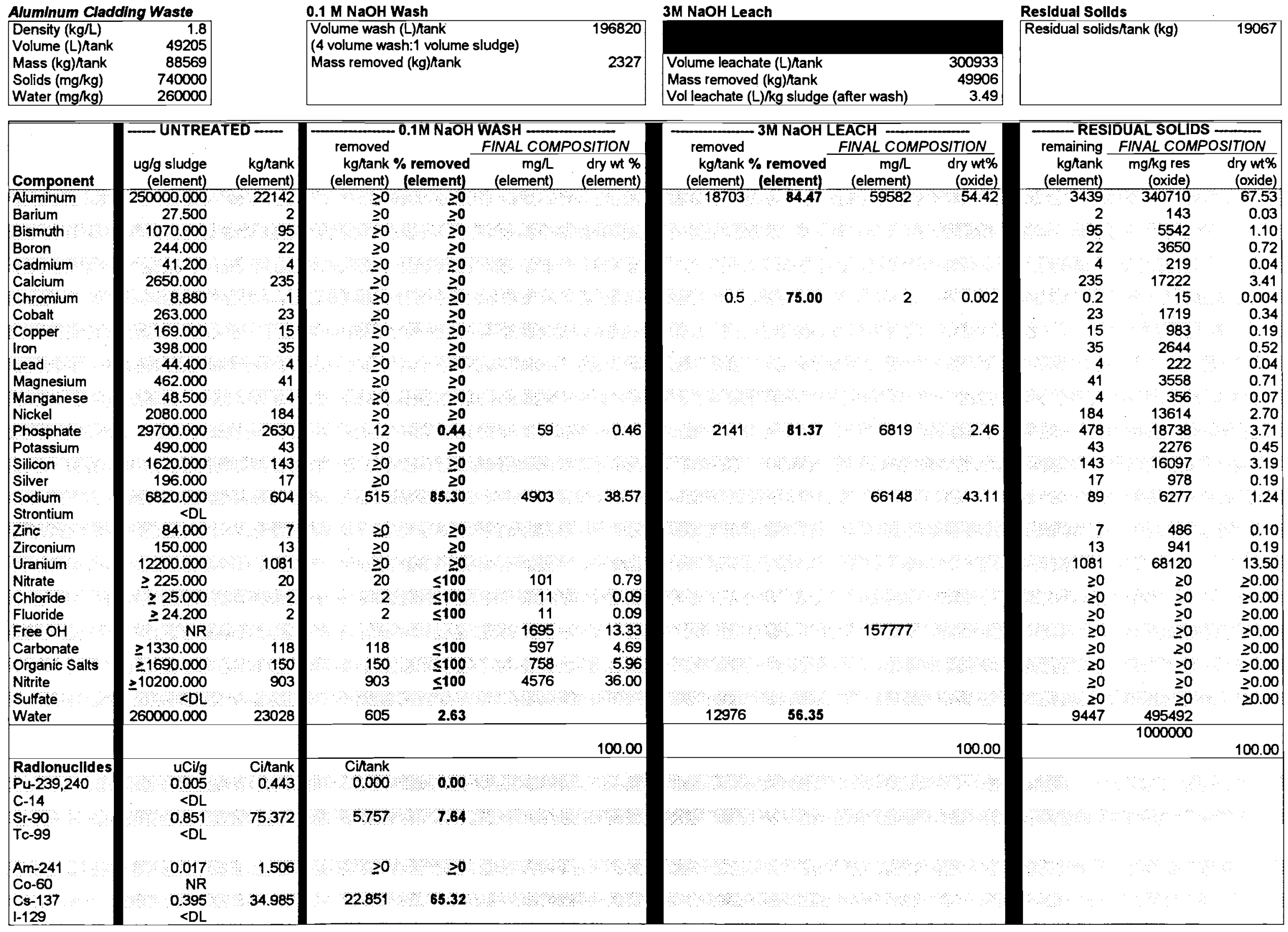




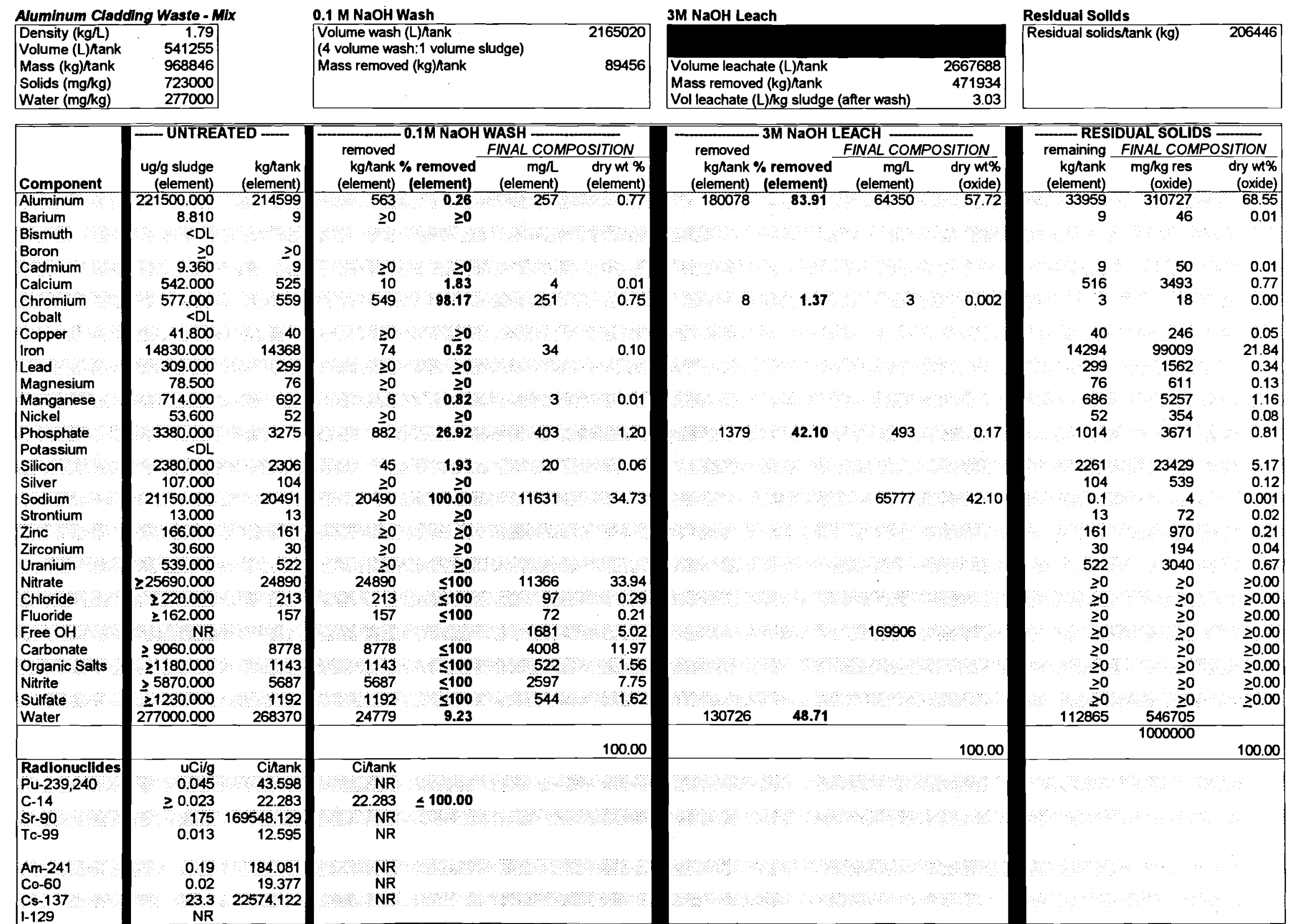




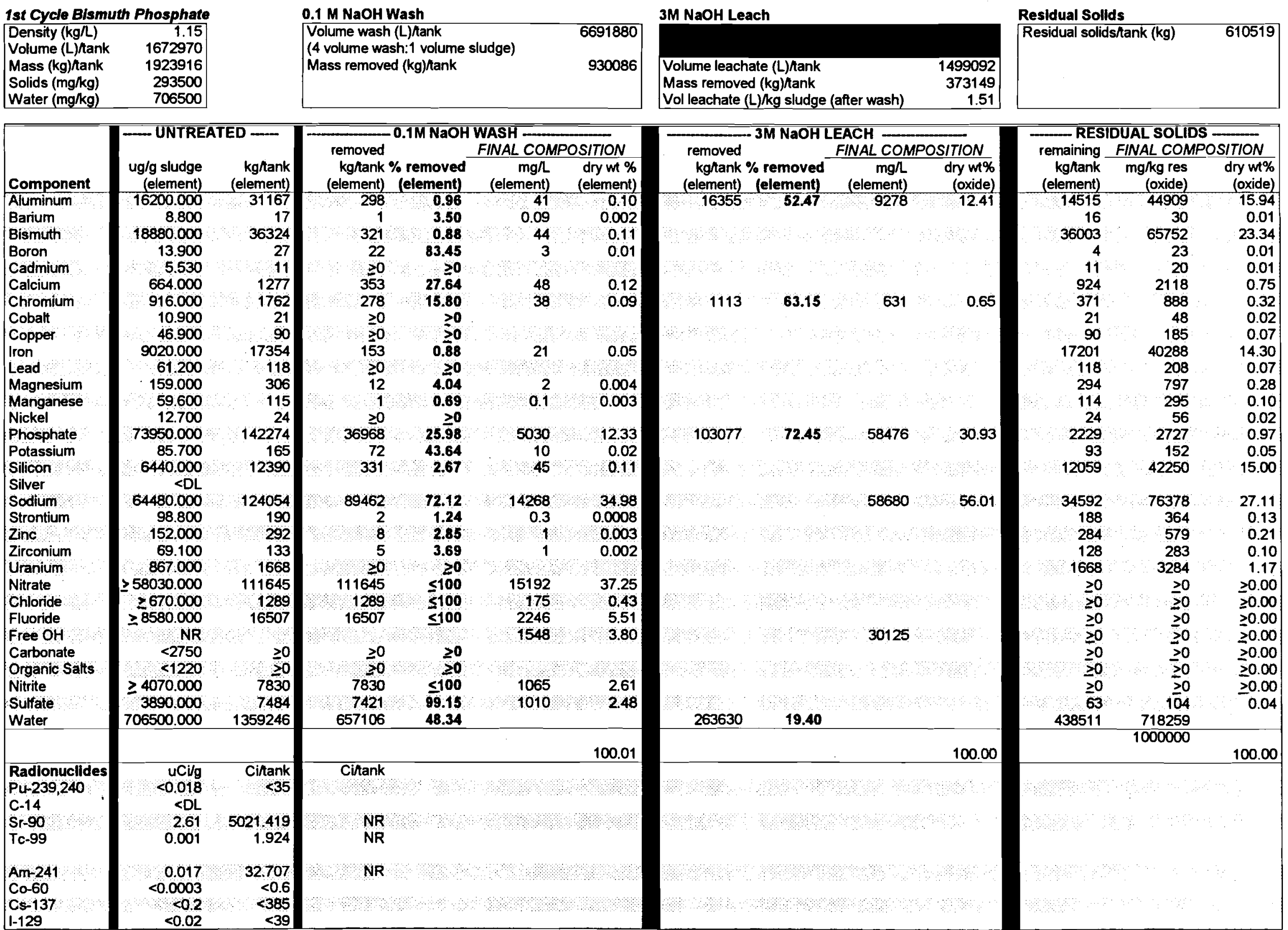


1st Cycle BIPO4 - Al Cladding Waste Density (kg/L)

Volume (L)Mank 114685

Mass (kg)hank $\quad 1146855$

Mass (kg)/ank 1720283

Solids (mg/kg)

741700

Water $(\mathrm{mg} / \mathrm{kg}) \quad 258300$
$0.1 \mathrm{M}$ NaOH Wash

Volume wash (L) hank

(4 volume wash: 1 volume sludge)

Mass removed $(\mathrm{kg})$ /tank

4587420

4587420

432954
$3 \mathrm{M}$ NaOH Leach

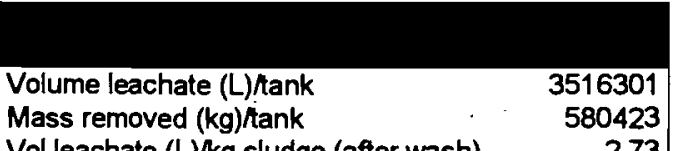

Residual Sollds

Residual solids/tank (kg) $\quad 525851$

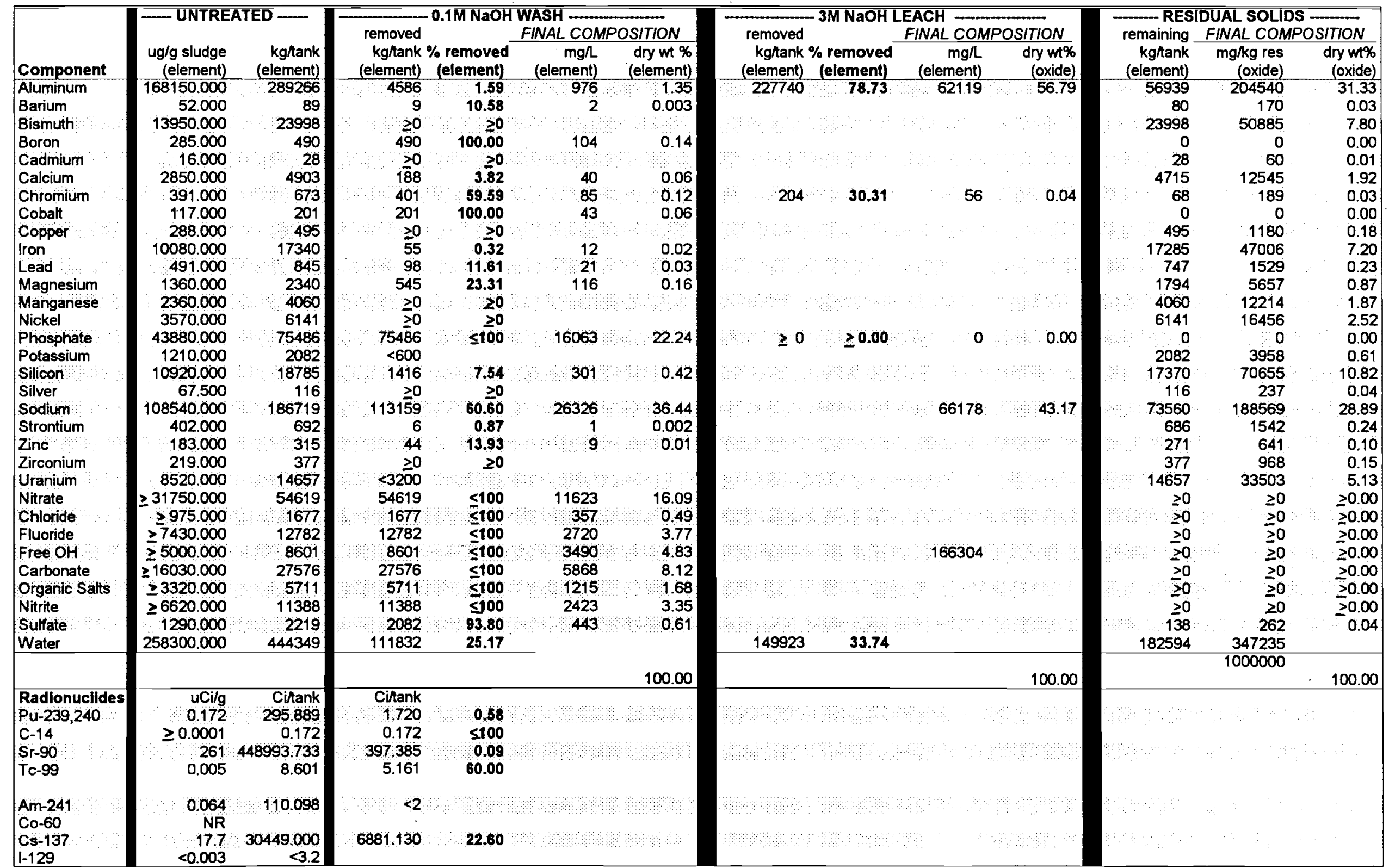


1st Cycle BIPO4 - Uranlum Recovery Density (kg/L) Volume (L)Mank $\quad 2615435$ Mass (kg)hank $\quad 3138522$

Solids $(\mathrm{mg} / \mathrm{kg}$ ) 421750 578250
$0.1 \mathrm{M}$ NaOH Wash

Volume wash (L)/tank

Mass removed $(\mathrm{kg})$ hank

1913960

\begin{tabular}{|lr|}
\hline Volume leachate (L)Mank & 2191832 \\
Mass removed $(\mathrm{kg})$ Mank & 364288 \\
Vol leachate (L) $/ \mathrm{kg}$ sludge (after wash) & 1.79 \\
\hline
\end{tabular}

Residual Sollds

Residual solids/tank (kg) $\quad \mathbf{8 4 7 8 4 8}$

Vol leachate (L) $/ \mathrm{kg}$ sludge (after wash) $\quad 1.79$

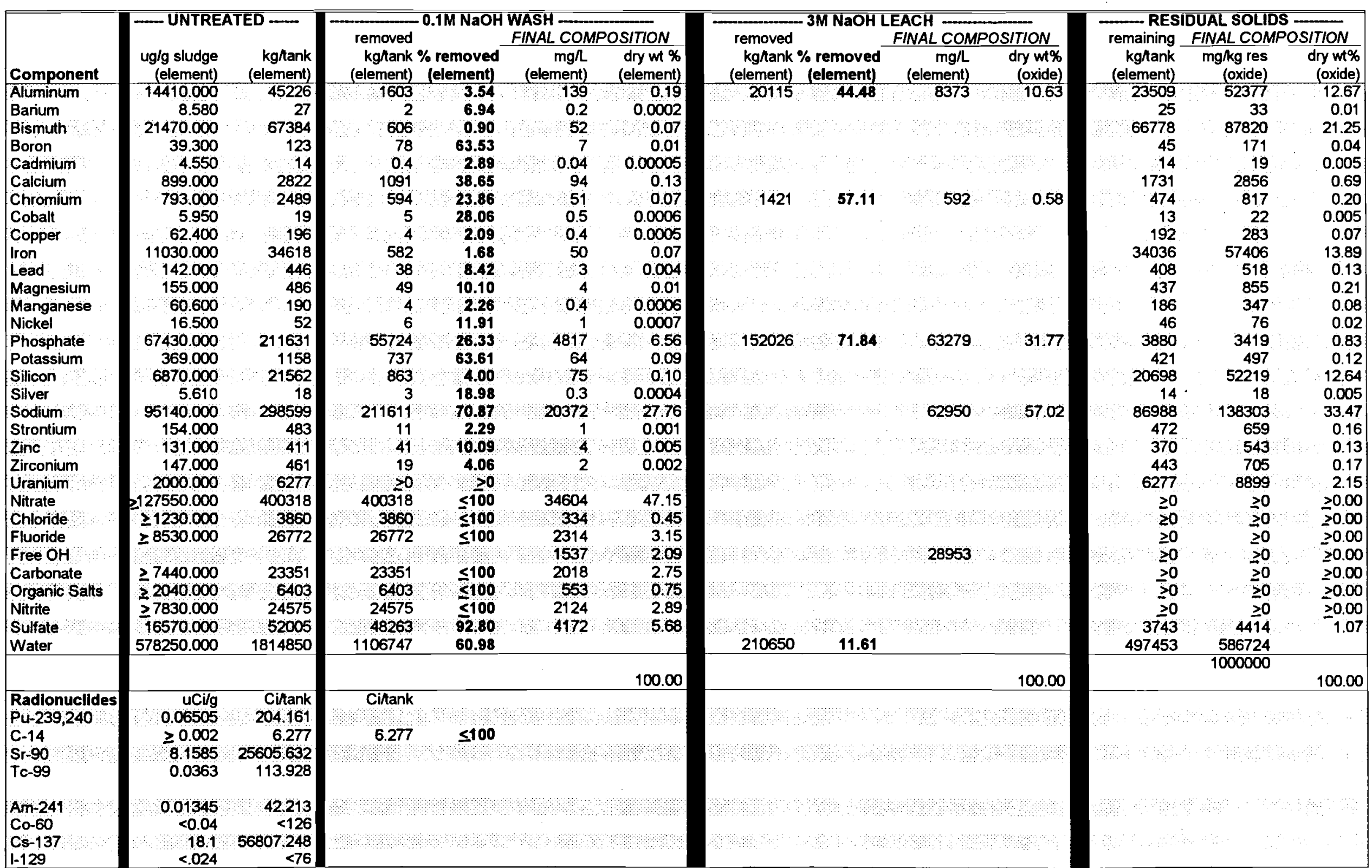


1 st Cycle BIPO4 - Evaporator Bottoms Density (kg/L) Volume (L)hank 1.64 Mass (kg)hank 2653285 Solids (mg/kg) $\quad 565000$ 435000
$0.1 \mathrm{M}$ NaOH Wash Volume wash (L)/tank (4 volume wash:1 volume sludge)

Mass removed (kg)Mank
3M NaOH Leach

0613140

1905141

\begin{tabular}{|lr|}
\hline & \\
\hline Volume leachate (L)/ank & 4467715 \\
Mass removed (kg)/ank & 409824 \\
Vol leachate (L)/kg sludge (after wash) & 1.83 \\
\hline
\end{tabular}

Resldual Sollds

Residual solids/tank (kg)

2212379

.83

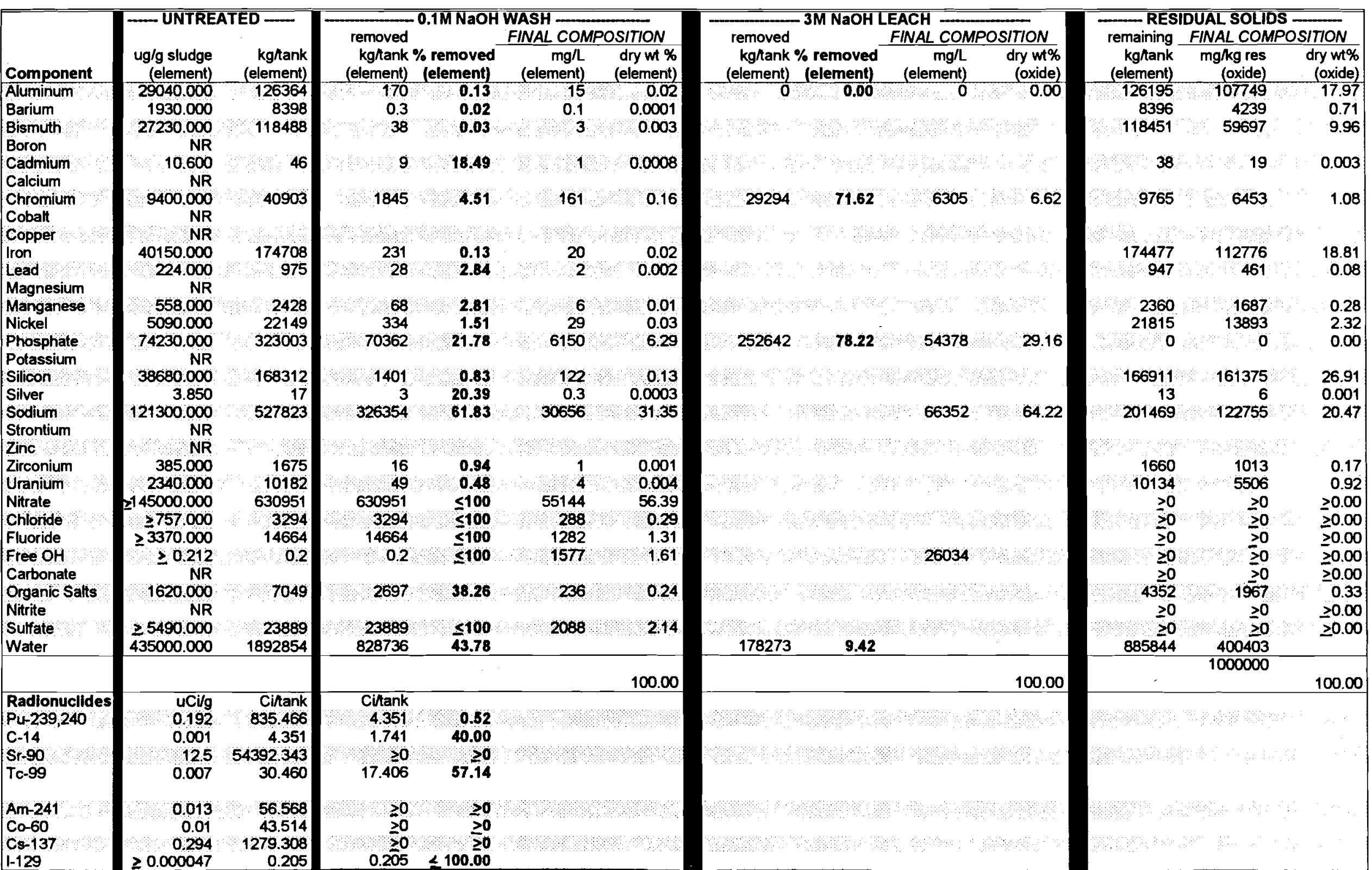


2nd Cycle BIPO4 - Tank 5-6, B-Plant Density (kg Volume (L)hank $\quad 1.303$

Solids (mg/kg)

Water (mg/kg)

1934135

2520178

398510

601490
$0.1 \mathrm{M} \mathrm{NaOH}$ Wash

Volume wash (L)hank

(4 volume wash:1 volume sludge)

Mass removed (kg) hank
$3 \mathrm{M} \mathrm{NaOH}$ Leach

7736540

1902715
Residual Sollds

Residual solidshank (kg)

523027

olume leachate (L)Mank

Mass remo

$\begin{array}{lr}\text { Vol leachate (L) } / \mathrm{kg} \text { sludge (after wash) } & 1.21\end{array}$

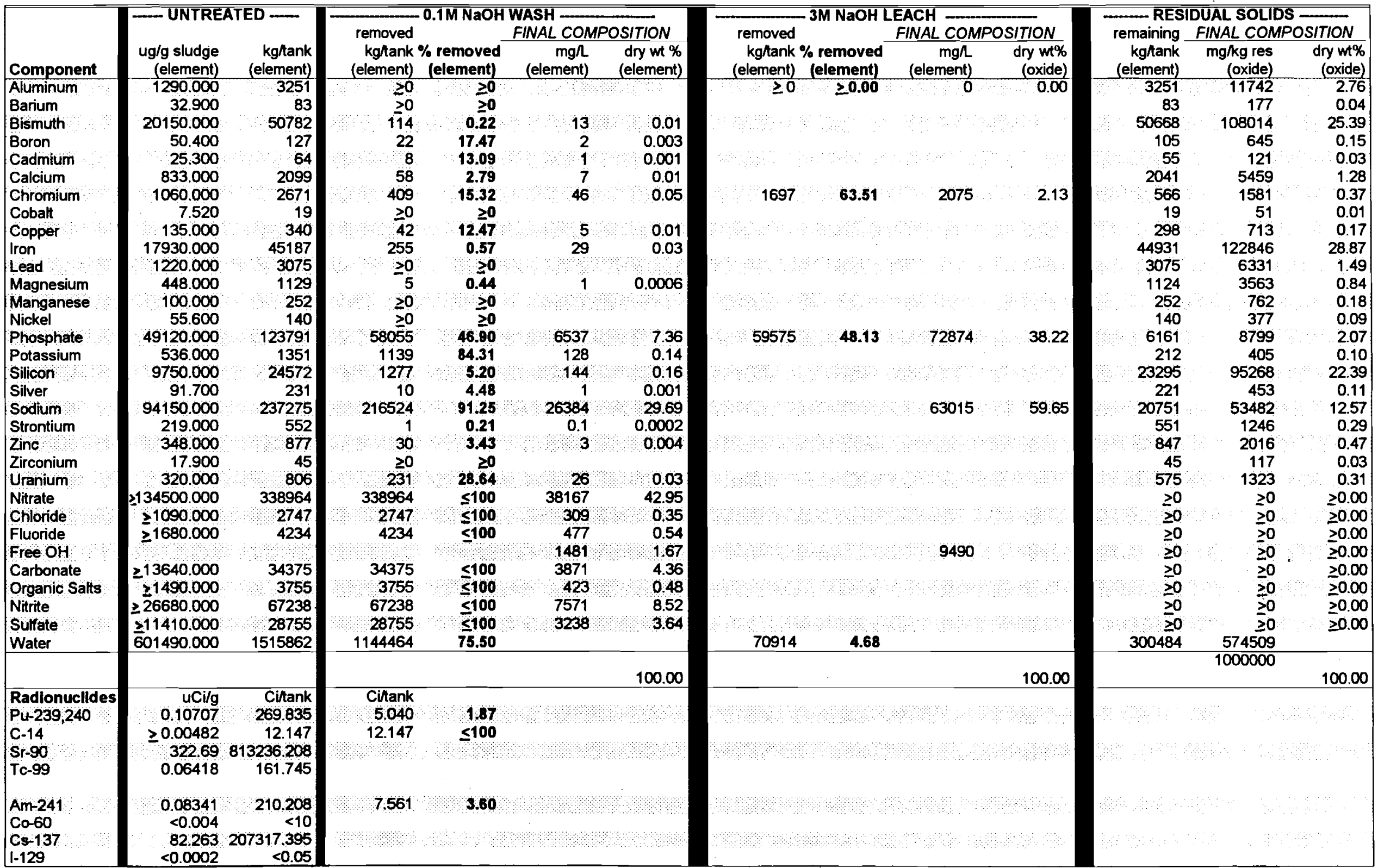


2nd Cycle BIPO4 - 224 Cycle BIPO4 Density (kg/L)

Volume (L)hank 3364865

Mass (kg)/tank $\quad 3701352$

Solids (mg/kg)

239800

\begin{tabular}{l}
239800 \\
760200 \\
\hline
\end{tabular}
0.1 M NaOH Wash

Volume wash (L)hank

(4 volume wash:1 volume sludge)

Mass removed (kg)/Aank
3M NaOH Leach

13459460

1729332

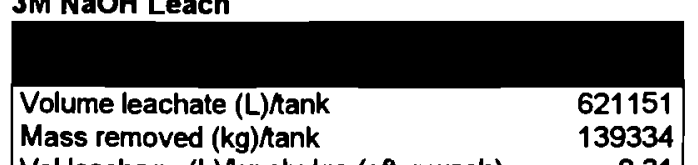

Residual Solids

Residual solids/tank (kg)

1769815

\begin{tabular}{lr} 
Mol leachage $(L) / \mathrm{kg}$ sludge (after wash) & 0.31 \\
\hline
\end{tabular}

\section{- UNTREATED -}

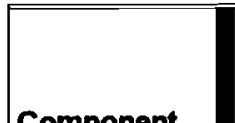

Aluminum

Barium

Boron

Lanthanum

Calcium

Chromium

Cobalt

Copper

Iron

Lead

Magnesium

Manganese

Nickel

Phosphate

Potassium

Silicon

Silver

Sodium

Strontium

Zine.

Zirconium

Uranium

Nitrate

Chloride.

Fluoride

Free $\mathrm{OH}$

Carbonate

Organic Salts

Nitrite

Sulfate

Water

Radionuclides

Pu 239,240

C-14

Sr-90

Tc-99

Am-241

Co-60

Cs-137

1-129

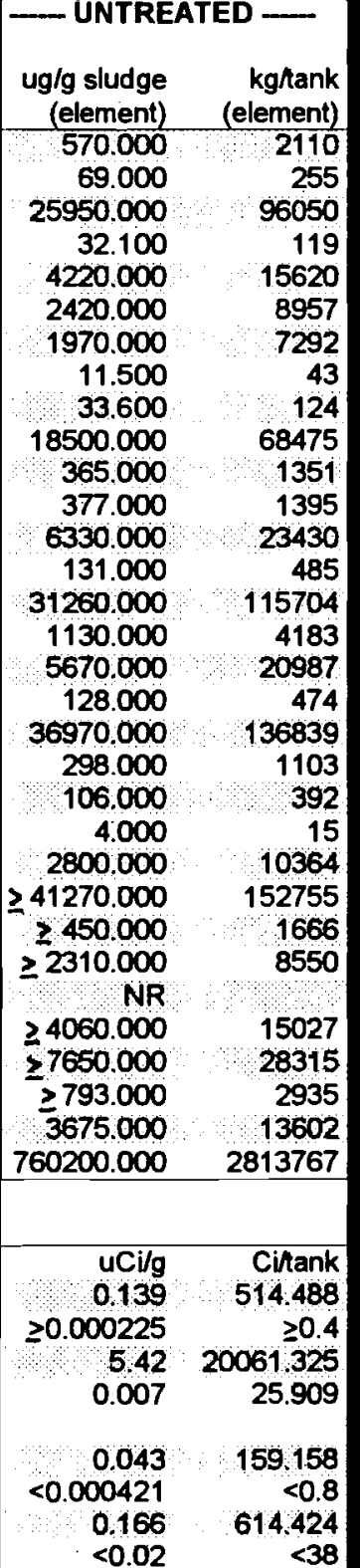

\section{M NaOH LEACH}

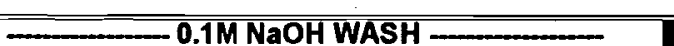

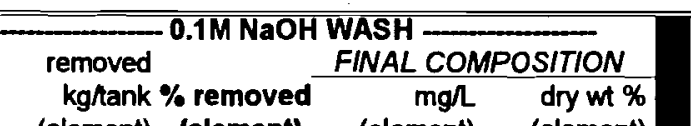
(element) (element) (element) (element)

\begin{tabular}{rrrr|}
\hline 40 & 1.91 & 3 & 0.01 \\
2 & 0.65 & 0.1 & 0.0004 \\
747 & 0.78 & 51 & 0.16
\end{tabular}

$\begin{array}{lr}747 & 0.78 \\ 15 & 12.69\end{array}$

$\begin{array}{rr}15 & 12.69 \\ 41 & 0.26\end{array}$

$\begin{array}{rr}41 & 0.26 \\ 228 & 2.54\end{array}$

$807 \quad 11.07$

$3 \quad 7.11$

1.19

0.69

1.88

0.97

0.39

1.30

54.51

63.63

10.08

$\begin{array}{cr}2116 & 10.08 \\ .3 & 0.56\end{array}$

$\begin{array}{cc}121960 & 89.13 \\ 7 & 0.66 \\ 1 & 0.88\end{array}$

0.28

20.00

$\begin{array}{rr}51 & 0.16 \\ 1 & 0.003\end{array}$

removed

kgtank \% removed

FINAL COM

COMPOSITION

\begin{tabular}{rrrr}
\multicolumn{2}{l}{$\begin{array}{r}\text { kg/tank \% removed } \\
\text { (element) }\end{array}$} & $\begin{array}{r}\mathrm{mg} / \mathrm{L} \\
\text { (element) }\end{array}$ & $\begin{array}{r}\text { dry wt\% } \\
\text { (element) }\end{array}$ \\
\hline$\geq 0$ & $\geq 0.00$ & 0 & 0.00
\end{tabular}

- RESIDUAL SOLIDS

remaining FINAL COMPOSITION

kghank mg/kg res

dry wt\% $\begin{array}{rrr}\text { (element) } & \text { (oxide) } & \text { (oxide) } \\ 2069 & 2209 & 1.04\end{array}$

\begin{tabular}{rrr|}
2069 & 2209 & 1.04 \\
254 & 160 & 0.08 \\
95303 & 60042 & 28.22
\end{tabular}

$\begin{array}{rrr}95303 & 60042 & 28.22 \\ 104 & 189 & 0.09\end{array}$

$\begin{array}{rrrr}104 & 18579 & 12588 & 5.92\end{array}$

$\begin{array}{rrr}15579 & 12588 & 5.92 \\ 8729 & 6900 & 3.24\end{array}$

0.0007

0.0003

$\begin{array}{ll}0.1 & 0.0003 \\ 32 & 0.10\end{array}$

$2 \quad 0.01$

$\begin{array}{rr}1 & 0.003 \\ 6 & 0.02\end{array}$

$0.4 \quad 0.001$

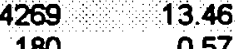

$\begin{array}{ll}180 & 0.57\end{array}$

$0.2 \quad 0.0006$

$10350 \% 32.64$

$0.5 \quad 0.002$

0.0002
0.0006

20
152755

$\begin{array}{lll}\geq 0 & 10339 & 32.60\end{array}$

1666

$\leq 100$
$\leq 100$

113

32.60
0.36

$\begin{array}{ll}579 & 1.82\end{array}$

$1549 \lcm{4.88}$

$\begin{array}{llll}15027 & \leq 100 & 1017 & 3.21 \\ 28315 & <100 & 1917 & 6.04\end{array}$

2935

199

6.04
0.63

$\begin{array}{rrr}13129 & 96.52 & 889\end{array} 2.80$

$\begin{array}{lll}1314639 & 46.72 & \\ & & 100.00\end{array}$

100.00

.

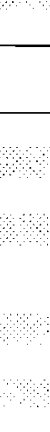

105922

3.76

24133

$1621 \quad 1339$ 
224 (Concentration) Cycle BIPO4 Density (kg/L)

Volume (L)hank 1.25

Mass (kg)tank

Solids (mg/kg)

Water (mg/kg)

604000
$0.1 \mathrm{M} \mathrm{NaOH}$ Wash

Volume wash (L)hank

(4 volume wash: 1 volume sludge)

Mass removed (kg)hank
$3 \mathrm{M} \mathrm{NaOH}$ Leach

4178640

362112
Residual Sollds

Residual solidskank (kg) $\quad 988195$

me leachate (L) Mank 330440

Mass removed (kg)hank 9010

Vol leachate (L) $/ \mathrm{kg}$ sludge (after wash) $\quad 0.35$

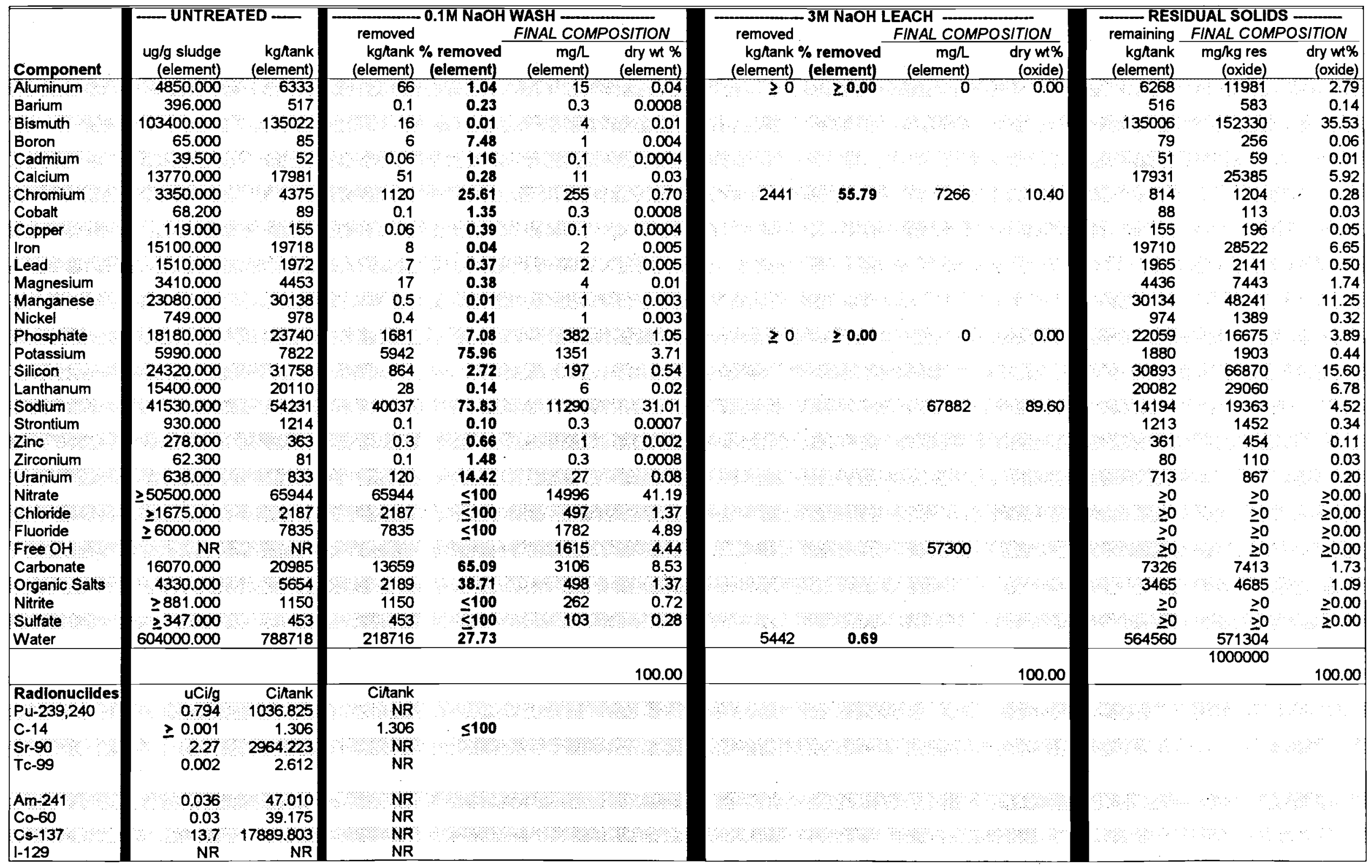


PUREX - PUREX Sludge SUpematant Density (kg/L) $\quad 1.39$ Volume (L) Kank Mass (kg)/tank $\quad 1362638$ Solids (mg/kg) $\quad 437160$ 562840
$0.1 \mathrm{M}$ NaOH Wash (4 volume wash:1 volume sludge)

Mass removed $(\mathrm{kg})$ /tank
Volume wash (L)/tank
$3 \mathrm{M} \mathrm{NaOH}$ Leach

3921260
Volume leachate (L)hank $\quad \mathbf{6 2 3 8 7 3}$

Mass removed $(\mathrm{kg})$ /tank 2784

Residual Solids

Residual solids/ank (kg) 1296082

Vol leachate (L) $/ \mathrm{kg}$ sludge (after wash) $\quad 0.51$

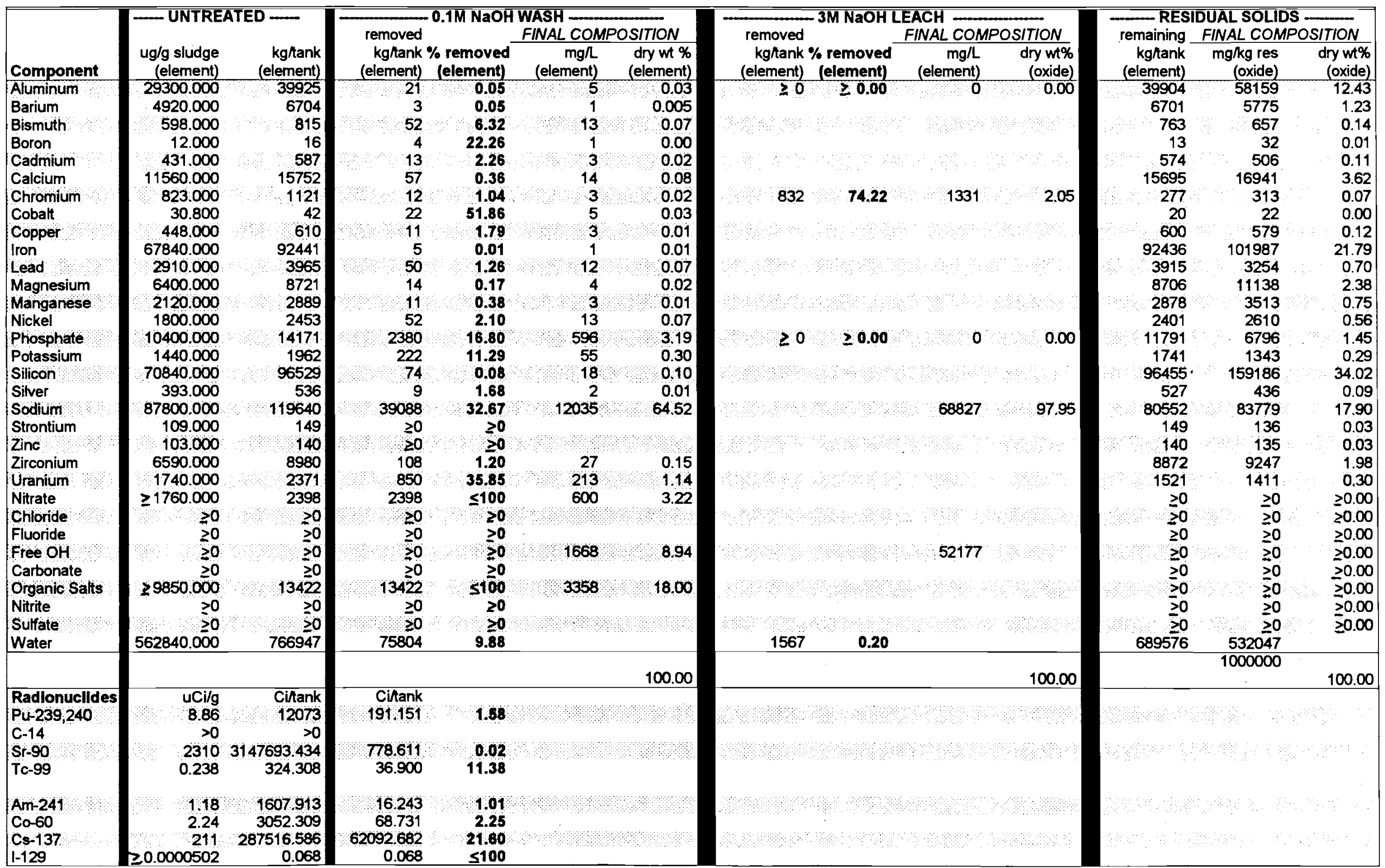


Uranium Recovery - Dlatomaceous Earth $0.1 \mathrm{M} \mathrm{NaOH}$ Wash

Density (kg/L)

Volume (L) tank

Mass $(\mathrm{kg})$ ) hank

Solids (mg/kg)

Water $(\mathrm{mg} / \mathrm{kg})$
Volume wash (L) Mank

(4 volume wash:1 volume sludge)

Mass removed $(\mathrm{kg})$ /tank

257380

64345

88153

638500

361500
$3 \mathrm{M} \mathrm{NaOH}$ Leach

39095
Volume leachate (L) hank

Mass removed $(\mathrm{kg})$ ) hank

Vol leachage (L) $/ \mathrm{kg}$ sludge (after wash)
Residual Sollds

Residual solids/tank (kg) $\quad 44824$

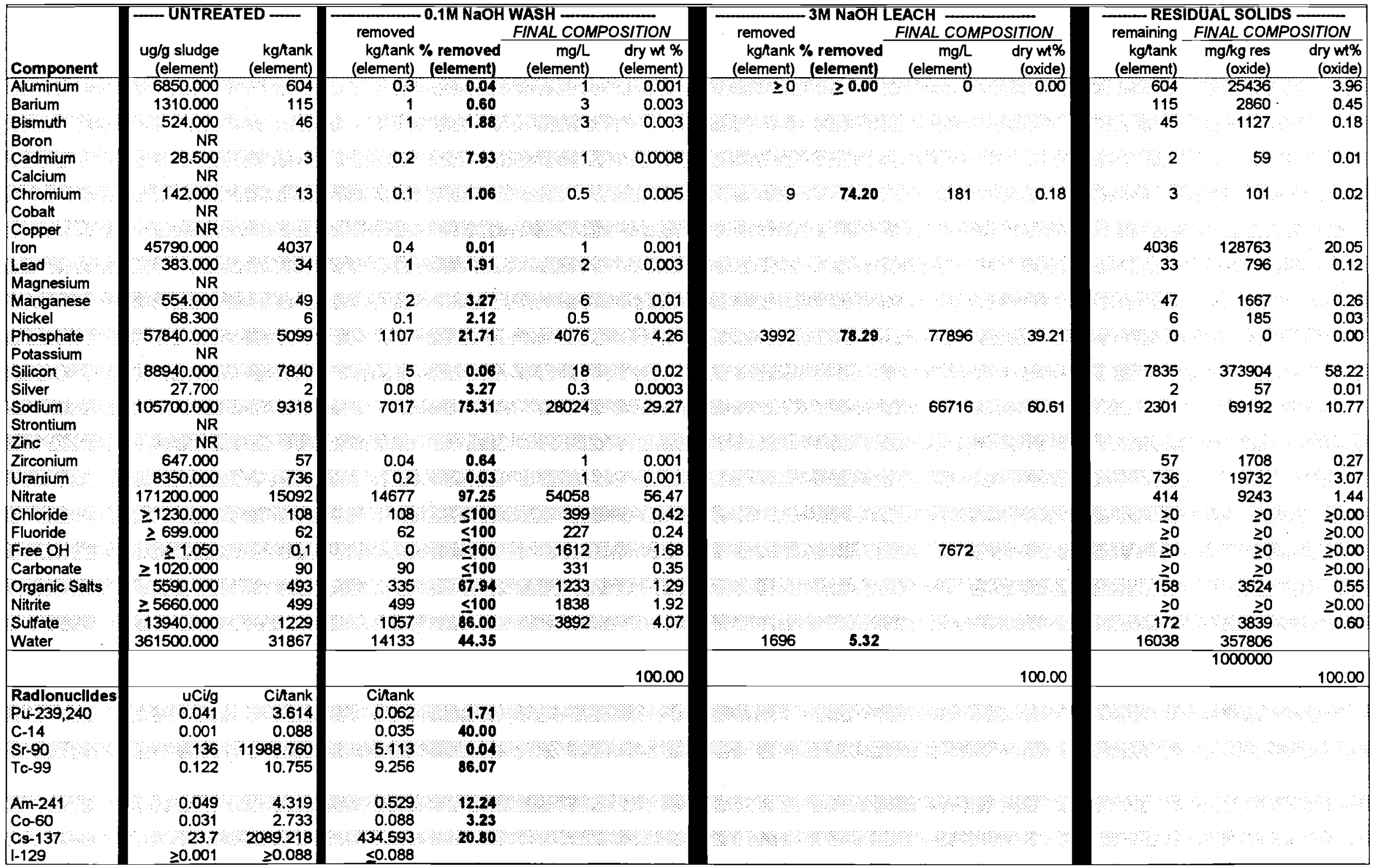


Uranlum Recovery - CW - Portand Cement 0.1 M NaOH Wash

Density (kg/L)

Volume (L)hank

Mass (kg)/rank

1.69
174110

294246

430000

570000

Solids (mg/kg)
Volume wash (L) hank

( 4 volume wash: 1 volume sludge)

Mass removed (kg)/ank

696440
$3 \mathrm{M} \mathrm{NaOH}$ Lach

118006

Volume leachate (L) Mank 175971

Mass removed (kg) Mank

Vol leachate $(\mathrm{L}) / \mathrm{kg}$ sludge (after wash) $\quad 1.00$

Residual Solids

Residual solidsıank (kg) 167500

—UNTREATED _-

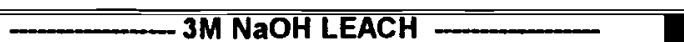

RESIDUAL SOLIDS -.. removed $\%$ removed $\frac{\text { FINAL COMPOSITION }}{\mathrm{mg} / \mathrm{L}}$ (element) (element

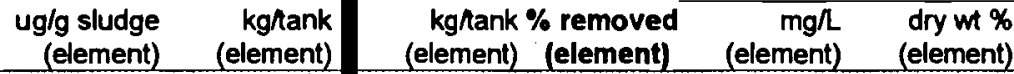

Component Aluminum Barium Bismuth

Boron

Cadmium

Calcium

Chromium

Cobalt

Copper

Iron

read

Magnesium

Manganese

Nickel

\& Phosphate

Silicon

Sodium

Strontium

Zinc

Zirconium

Uranium

Nitrate

Chloride

Fluoride

Free $\mathrm{OH}$

Carbonate

Organic Salts

Nitrite

Sulfate

Water

(element) (element)

33880.000

9969

776.000

5.300

44.300

6210.000

8650.000

0.570

27,300
6250.000

6250.000

563.000

3250.000

1490.000

147.000

59750.000

1440.000

41470.000

25.800

1100000.000

56.200

143.000

335.000

4410.000

$\geq 33250.000$

$\geq 33250.000$
NR

1298
9784

$\geq 3600.000$

000 $\quad 1059$

228
2
13

13
1827

2545

0.2

1839

166

956

43

17581

12202

8

32367

$\begin{array}{rr}276 & 0.39 \\ 0.5 & 0.0007\end{array}$

32651

0.3

0.79
0.001

$\begin{array}{rrr}1.83 & 5 & 0.01 \\ 100.00 & 2 & 0.002\end{array}$

727

1.41

1.75

100.00

10.26

0.04

7.55

0.12

1.73

17.79

77.27

54.55

72.09

1.27

$\begin{array}{lll}1.77 & 2 & 0.003\end{array}$

0.06

1
12811 $\quad \begin{array}{r}0.001 \\ 18.28\end{array}$

$9784 \leq 100$

18.28

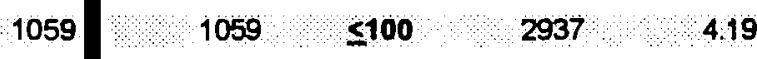

$\$ 6820.000 \quad 2007$

$2007, \leq 100 \quad 2628 \quad 3.75$

$\begin{array}{ll}\text { NR } & \\ 570000.000 & 167720\end{array}$

6726

40.10

3.7

Radionuclldes Pu-239,240

C-14

Sr-90

Tc-99

Am-241

Co-60

Cs-137

1-129

$\begin{array}{rr}u \text { ui } / g & \text { Cinank } \\ 0,28 & 82.389 \\ \geq 0.001 & 0.294 \\ 137 & 40311.688 \\ \geq 0.034 & 10.004 \\ & \\ 0.994 & 292.480 \\ 0.157 & 46.197 \\ 54.1 & 15918.703 \\ <0.000037 & <0.01\end{array}$

100.00

3635

$\geq 0.00$

(oxide)

remaining FINAL COMPOSITION

kghank mg/kg res dry wt\%

(element) (oxide) (oxide)

$\begin{array}{rrr}9548 & 107682 & 25.52 \\ 941 & 6275 & 1.49\end{array}$

$\begin{array}{rrr}941 & 6275 & 1.49 \\ 224 & 1492 & 0.35 \\ 0 & & 0.00\end{array}$

$\begin{array}{rrr}0 & 0 & 0.00 \\ 12 & 82 & 0.02\end{array}$

$\begin{array}{lll}1801 & 15047 & 3.57\end{array}$

$\begin{array}{lll}625 & 5457 & 1.29\end{array}$

$\begin{array}{rrr}0 & 0 & 0.00 \\ 7 & 54 & 0.01\end{array}$

$\begin{array}{lll}1838 & 15695 & 3.72\end{array}$

$\begin{array}{lll}153 & 985 & 0.23\end{array}$

$\begin{array}{lll}955 & 9454 & 2.24 \\ 331 & 4069 & 0.96\end{array}$

$\begin{array}{rrr}431 & 4069 & 0.96 \\ 36 & 299 & 0.07\end{array}$

$\geq 0 \geq 0.00 \quad 0 \quad 0.00$

$67604 \quad 85.65$

$193 \quad 1150$

$11992 \quad 153136$

$\begin{array}{rrr}9033 & 72698 & 0.01\end{array}$

$\begin{array}{rrr}16 & 115 & 17.23 \\ 42 & 313 & 0.03\end{array}$

$\begin{array}{lll}42 & 313 & 0.07 \\ 97 & 781 & 0.19\end{array}$

$1297 \quad 9306 \quad 221$

$\geq 0 \quad \geq 0 \quad \geq 0.00$

$\geq 0 \quad \geq 0 \quad \geq 0.00$

60209

Cinank
3.237

$\begin{array}{rr}0.294 & \leq 100 \\ 700305 & 174\end{array}$

$10.004 \leq 100$

$3.531 \quad 1.21$

$11.770 \quad 25.48$

$13270.490 \quad 83.36$ 
Uranlum Recovery - 1st Cycle BIPO4 (F)

\begin{tabular}{|lr|}
\hline Density (kg/L) & 1.7 \\
Volume (L)/ank & 775925 \\
Mass (kg)/ank & 1319073 \\
Solids (mg/kg) & 465155 \\
Want & 534845 \\
\hline
\end{tabular}

534845
$0.1 \mathrm{M}$ NaOH Wash

Volume wash (L)/tank

(4 volume wash:1 volume sludge)

Mass removed (kg)/tank

3103700

741481

$3 \mathrm{M} \mathrm{NaOH}$ Leach

\begin{tabular}{|lr|}
\hline Volume leachate $(\mathrm{L})$ /)ank & 693342 \\
Mass removed $(\mathrm{kg})$ /ank & 86196 \\
Vol leachate (L)/kg sludge (after wash) & 1.20 \\
\hline
\end{tabular}
Residual Solids

Residual solidstank (kg) 494634

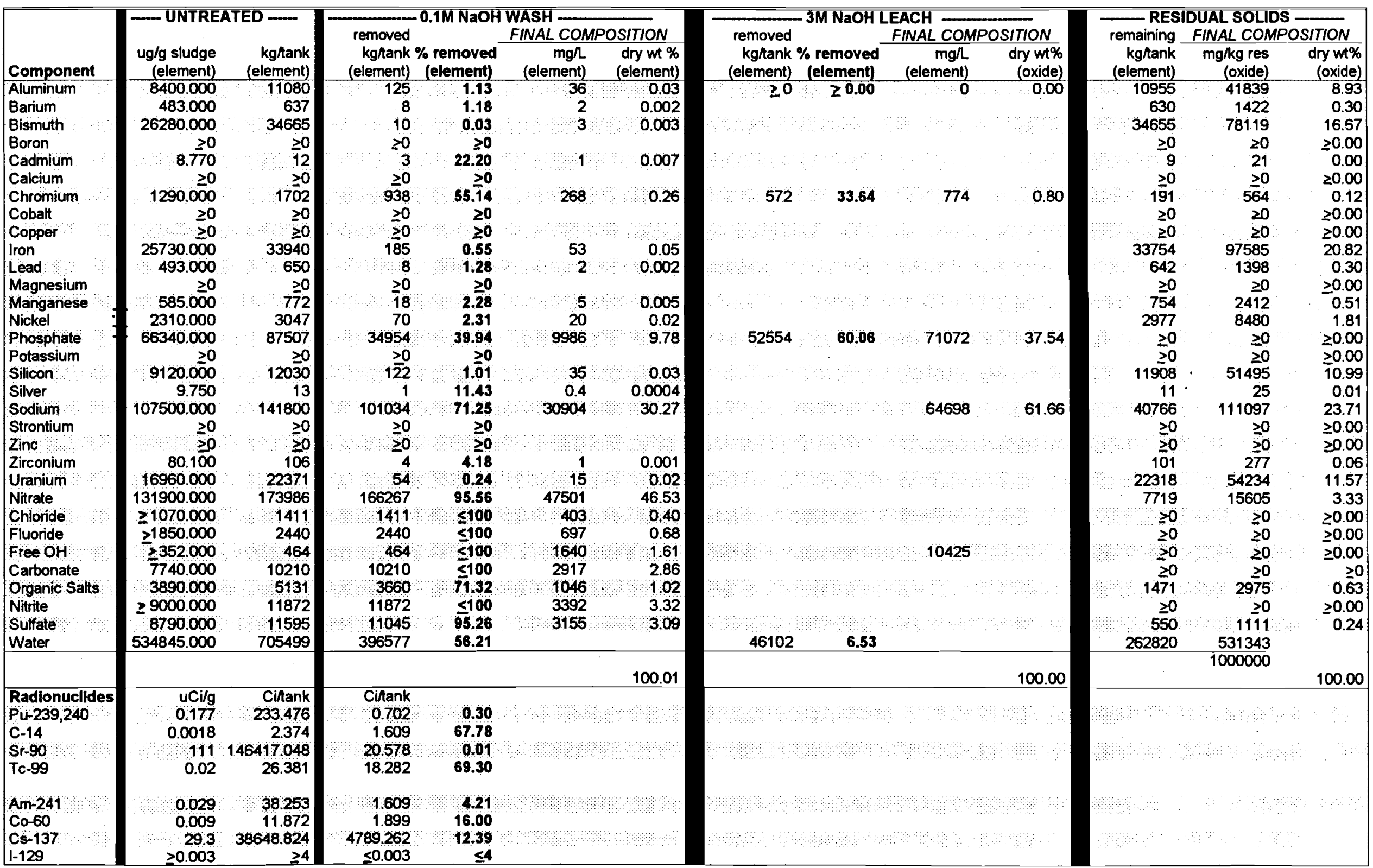




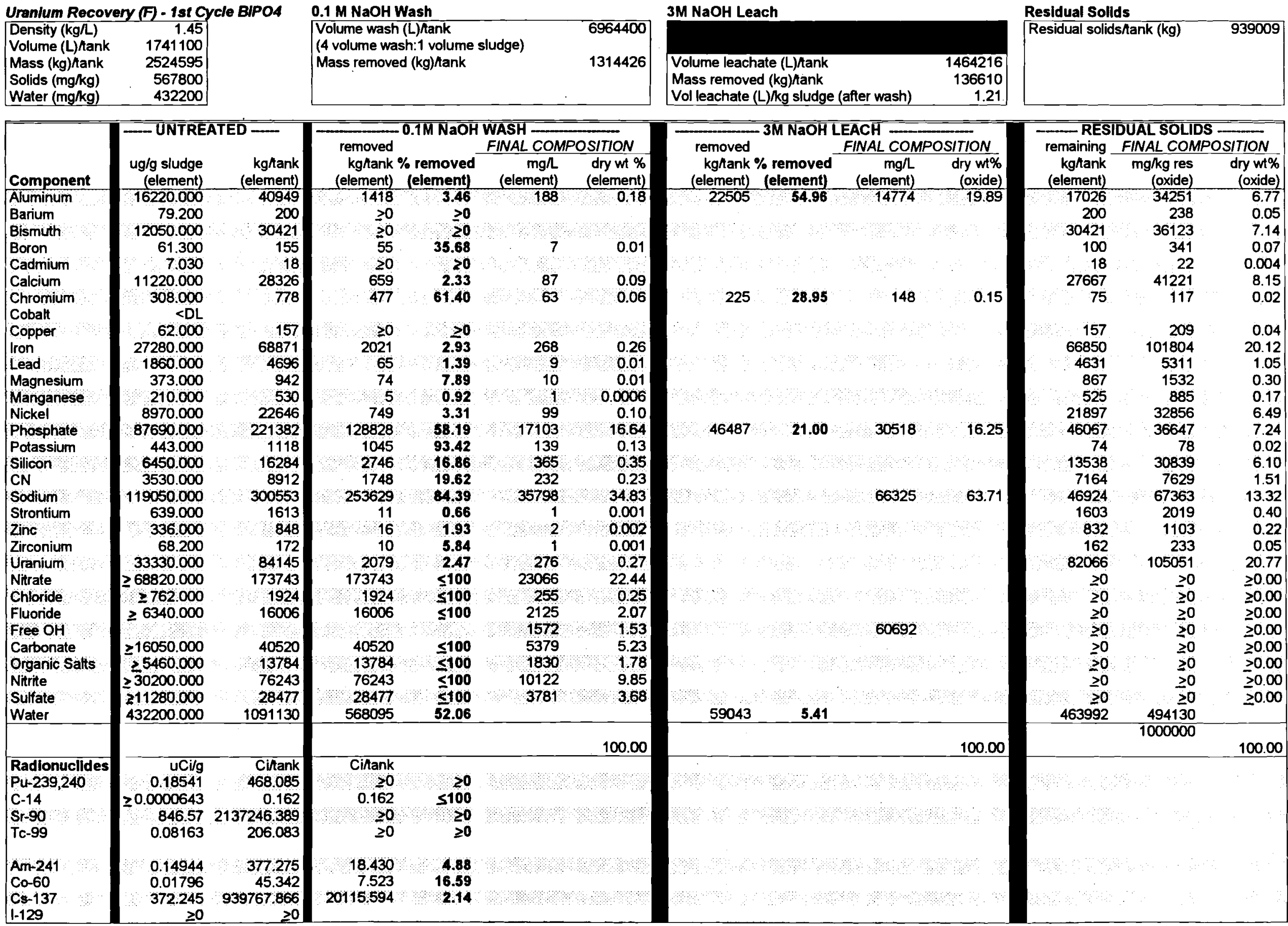


REDOX

\begin{tabular}{|lr|}
\hline Density (kg/L) & 1.5 \\
Volume (L)/Mank & 2626790 \\
Mass (kg)/ank & 3940185 \\
Solids (mg/kg) & 652000 \\
Water (mg/kg) & 348000 \\
\hline
\end{tabular}

$0.1 \mathrm{M} \mathrm{NaOH}$ Wash

Volume wash (L) Mank

(4 volume wash:1 volume sludge)

Mass removed (kg)/tank
$3 \mathrm{M} \mathrm{NaOH}$ Leach

\begin{tabular}{|lr|}
\hline Volume leachate $(\mathrm{L})$ Mank & 5580706 \\
Mass removed $(\mathrm{kg})$ /ank & 1619345 \\
Vol leachate $(\mathrm{L}) / \mathrm{kg}$ sludge (after wash) & 3.19 \\
\hline
\end{tabular}

Vol leachate (L)/kg sludge (after wash) $\quad 3.19$
Residual Solids

Residual solids/tank (kg) $\quad 324120$

2189386

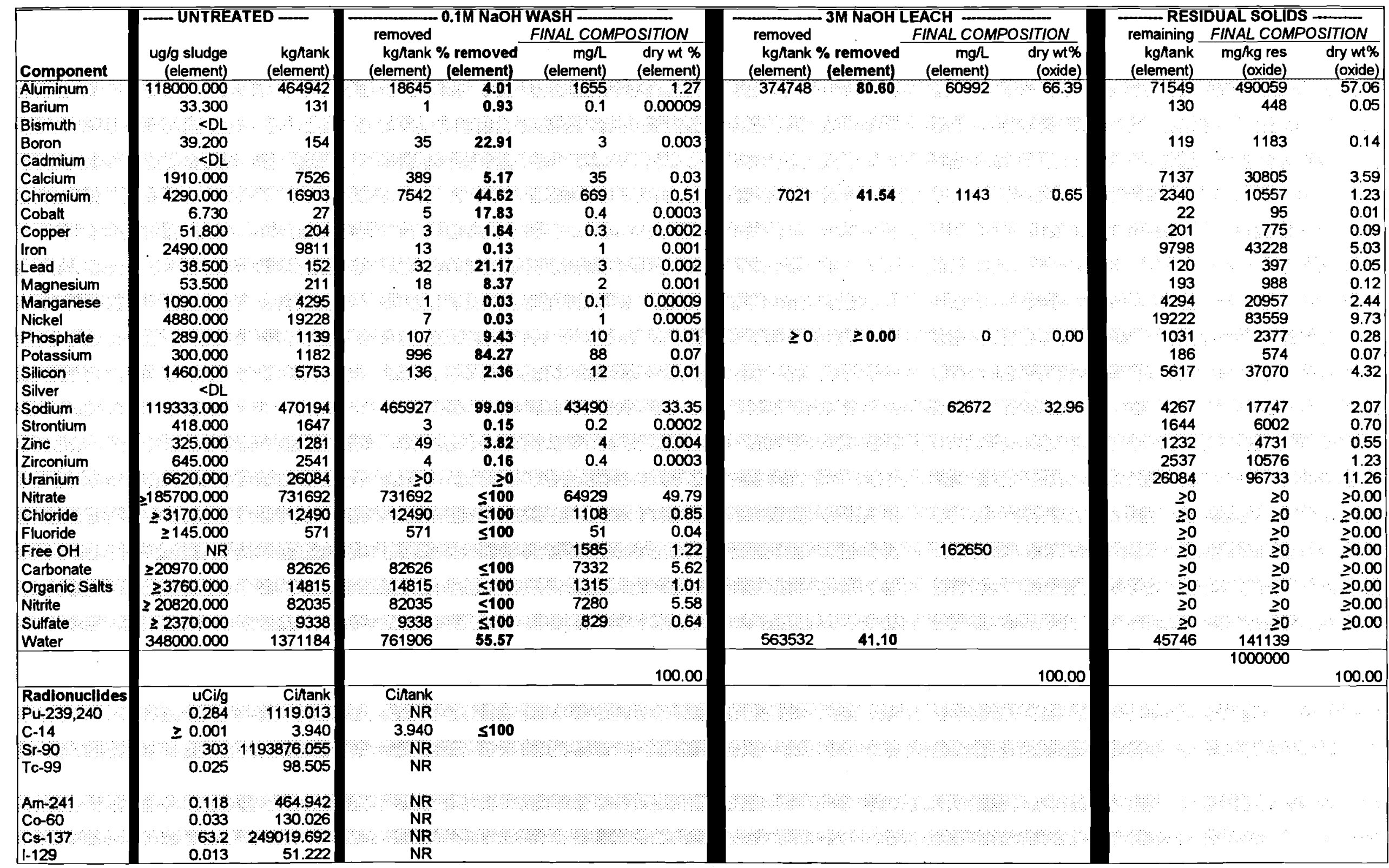




\subsection{References}




\subsection{References}

Boomer, K. D., et al. 1993. Tank Waste Technical Options Report, Appendix G. WHC-EP-0610 Rev. 0, Westinghouse Hanford Company, Richland, Washington.

Hanlon, B. M. 1994. Tank Farm Surveillance and Waste Status Summary Report for July 1994. WHC-EP-0182-75, Westinghouse Hanford Company, Richland, Washington.

Hill, J. G., and B. C. Simpson. 1994. The Sort on Radioactive Waste Type Model: A Method to Sort Single-shell Tanks Into Characteristic Groups. PNL-9814, Pacific Northwest Laboratory, Richland, Washington.

Lumetta, G. J., and B. M. Rapko. 1994. Washing and Alkaline Leaching of Hanford Tank Sludge: A Status Report. PNL-10078, Pacific Northwest Laboratory, Richland, Washington.

Lumetta, G. J., B. M. Rapko, C. D. Carlson, M. J. Wagner, R. J. Barrington. 1994. Sludge Treatment and Extraction Technology Development: Results of FY 1993 Studies. PNL-9387, Pacific Northwest Laboratory, Richland, Washington.

Stordeur, R. T., et al. 1986. Hanford Defense Waste Disposal Alternatives Engineering Support Data for the HDW-EIS. RHO-RE-ST-30P, Rockwell Hanford Operations, Richland, Washington. 

PNL-10512

UC-721

\section{Distribution}

No. of

Copies

$\underline{\text { OFFSITE }}$

12 DOE/Office of Scientific and

Technical Information

D. Wynne

EM-361, Trevion II

U.S. Department of Energy

12800 Middlebrook Road

Germantown, MD 20874

S. F. Agnew

Chemical Science \& Technology

Division

Los Alamos National Laboratory

Los Alamos, NM 87545

E. C. Beahm (2)

Martin Marietta Energy Systems, Inc.

P. O. Box 2008

Oak Ridge, TN 37831-6223

B. Z. Egan

Martin Marietta Energy Systems, Inc.

P. O. Box 2008

Oak Ridge, TN 37831-6223

R. King

Washington Department of Ecology

P. O. Box 47600

Olympia, WA $98594-7600$

H. Todosow

Brookhaven National Laboratory

32 Lewis Road, Building 130

Upton, NY 11973-5000
No. of

Copies

\section{ONSITE}

3 DOE Richland Operations

S. T. Burnum, R3-74

R. A. Gilbert, R3-74

P. E. Lamont, R3-74

17 Westinghouse Hanford Company

J. N. Appel, L6-29

S. A. Barker, L6-29

W. B. Barton, L4-75

A. L. Boldt, H5-49

K. D. Boomer, H5-49

T. M. Brown, R2-12

K. A. Gasper, L6-29

M. E. Johnson, B1-58

R. A. Kirkbridge, S4-58

M. J. Kupfer, H5-49

S. L. Lambert, H5-27

G. T. MacLean, S4-58

R. M. Orme, S4-58

L. W. Shelton, Jr., H5-49

B. C. Simpson, R2-12

D. J. Washenfelder, L4-75

R. A. Watrous, H5-27

42 Pacific Northwest Laboratory

G. S. Anderson, K7-94

E. G. Baker, P8-38

D. L. Blanchard, Jr., P7-25

B. C. Bunker, K2-45

N. G. Colton, K3-75 (5)

J. M. Creer, K9-80

A. R. Felmy, K9-77

Distr.1 
PNL-10512

UC-721

No. of

Copies

S. R. Gano, P8-38

J. G. Hill, K7-94

L. K. Holton, P7-43 (5)

E. O. Jones, K3-75

B. M. Johnson, K1-78

W. L. Kuhn, K2-21

D. E. Kurath, P7-43

J. P. LaFemina, K2-25 (5)

J. Liu, K2-44

G. J. Lumetta, P7-25
No. of

Copies

B. M. Rapko, P7-25

B. A. Reynolds, P7-19

W. G. Richmond, K6-51

L. J. Sealock, Jr., K2-10

J. T. Slankas, K9-81

J. L. Swanson, P7-25

K. D. Wiemers, P7-14

Publishing Coordination

Technical Report Files (5) 